INSTITUTE OF CHEMICAL TECHNOLOGY (UPV-CSIC)

\title{
Heterojunctions of defective graphenes with 2D materials and metal nanoplatelets: preparation and catalytic applications
}

\author{
Doctoral Thesis
}

Presented by:

Jinbao He

Supervisor:

Prof. Hermenegildo García Gómez

Dr. Ana Primo Arnau

Valencia, October 2018 



\section{Acknowledgements}

It has been 3 years since I arrived at Spain. Time went so fast. I still remember that the life in the first 6 months was like a dream for me, everything was new and made me exciting. I really enjoyed the life a lot during my stay here. For the research, I should say that there were some tough times when I lost direction, especially in the early stage. Thanks to the nice persons who appeared in my life during the past 3 years, finally I could go through these hard times and finish my PhD program on time and also had an unforgettable experience here.

At first, I would like to thank sincerely my thesis supervisors, Hermenegildo Garcia and Ana Primo. Herme, thank you very much for giving me the opportunity to work with you. The whole story begins from your acceptance. Also, thank you for guiding me into the chemistry world and your great passion on science motivated me a lot in the research. Your advice was always so important to help me move on when I was struck in the research problems. During these years for doctoral study, I have learned so many things from you. Ana, you are a so kind and patient supervisor, helping me a lot in my doctoral program and giving me lots of useful advice to carry out the experiments. You and Herme helped me so much in the research and also daily life during my stay in Spain and made everything easy for me.

Then, I want to express my thanks to all of my laboratory partners. Ivan, you taught me tons of things in the experimental skill, like my third supervisor. Besides, you helped me a lot in my daily life (introducing me to the running club and football team, helping me in the doctorate enrollment procedure, etc). Diego, thank you for the friendship and also being so patient to help me in photocatalytic experiments. Abde, you are the first person that introduced me to the group and helped me a lot in the enrollment procedure as well as the performance of organic reactions. Sonia, you are a so nice girl that I met in my second year for PhD, thank you for your kind encouragement during my hard time in the research and daily life as well. Amparo, you gave me the primal lessons in the synthesis of graphene when I began my work 
in the lab and it really helped me a lot. Josep, Pedro, thank you for your help in photoelectric experiments. Both of you behaved as expert in this area. Prof. Li, you always could give me useful advice in the research and show me the right direction from a very high point of view. Prof. Niu, thank you so much for some practical advice in the experiment skill.

Many thanks to the rest partners in the laboratory: Xisco, Alejandra (Madrid), LuLu, Cristina, Ester, Rocio, Marcos, Teresa, Alejandra, Elena, Aicha, Lu Peng, Yong Peng, Yue Sun, Javi, Bertrand, Nadia, Paula, Alberto, Giulio, Umberto, Sana, etc and the partners from department of chemistry: Juan Calros, Juan Fran, Andera, Herme, Juan Fran, Sergio, Belen, Mercedes, Murthy, Sapa, Ariadna, etc and also the other partners from ITQ: Marvin, Victor, Jaime, Ferran, Tong Qin, Chengeng Li, Zaher, Silvia, etc. Thank all of you for sharing the unforgettable time with me. Thanks a lot to Dr. Gao and Lichen Liu for your useful advice for my experiments at the beginning. In addition, I want to give my sincere thanks to Prof. Kian Ping Loh for accepting my 3-month internship in Department of Chemistry in National University of Singapore as well as to the group numbers (Zhongxin Chen, Cuibo Liu, Wei Liu, Kai Leng, Prof. Xie, Wei Tang, Xiaowei Wang, Haisen Xu, Zhen Pei, Yunxuan, Rachel, etc) for helping me in the experiments and sharing a short but unforgettable time together.

Besides, I want to give my thanks to the administrative staffs and technicians. To the administrative staffs in ITQ (Inma, Marcos, Natalia, Antonio, Maria, etc) and those in doctorate school, lots of my problems were solved efficiently with your help. Many thanks to the technicians in workshop (Rafa, Oscar, Álvaro, David, Paquito, Javier Pérez, etc) for the equipment maintenance, the technicians (Rosa, Marta Puche, Dolo, Adelina, Amparo, Maribel, Paticia, Jose Miguel, Maria Jesus, Fani, Estrella, Ale, Miguel, etc) for the sample characterization and the technicians in microscopy services of UPV (Merche, Manolo, Jose Luis, especial thanks to Alicia and Ximo for being so nice) for the help in the use of microscope.

I also would like to give thanks to all of my friends who make my life so wonderful in Spain. Firstly, to our English group (Hans, Bea, Anna, Eva, Ale, Maria, 
Rosella, Peng Lu, Sonia, Danie, Jose, Zeneida, etc), I always can have lots of fun with you. I will never forget the time that we spent together. Then, to the numbers in running club of UPV (Juan, Ester, Ivan, Diego, Juan calros, Josep, Sonia, etc), I should say it is a pity that I missed the half marathon with you twice due to my injured foot during that time. And to ITQ football team numbers (Javi, Miguel, Ivan, Marcelo, Pascual, Christian, Segio, Marvin, etc), we have good memories in the campus league of UPV. And to the Spanish language course numbers (Oscar, Hans, Anna, Stefano, etc), I had a really unforgettable time with you in the class, learning the language as well as the Spanish culture. Oscar, you are not only a funny teacher who can always make the class interesting but also a good friend in our life. Also, to my roommate, Tiemin Xuan, you are the first person who received me in Valencia and helped me a lot to get used to the life here.

The special thanks should be given to the China Scholarship Council (CSC) for the financial support in Valencia and to the staffs in education office of the Embassy of the People's Republic of China in Spain for their kind help.

Finally, I would like to thank my family to give me the support to study in Spain. Your encouragements always make me feel good to stay here. 



\section{Table of contents}

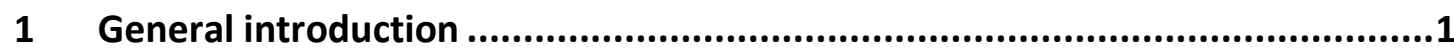

1.1 Graphene

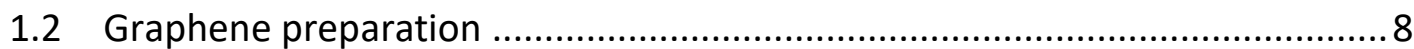

1.3 Defective graphenes by pyrolysis of biopolymers ....................................17

1.4 Heterojunctions of defective graphenes with 2D materials or metal

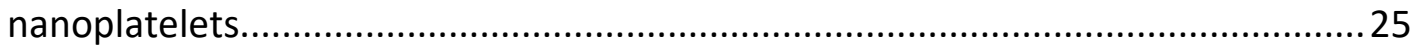

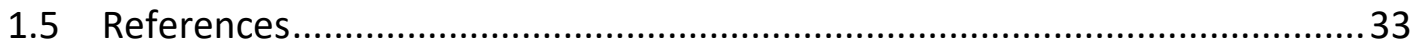

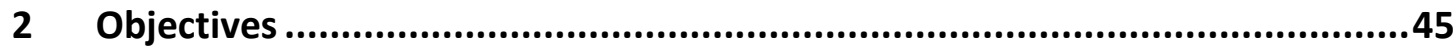

3 Quality improvement of defective graphene from biomass ........................49

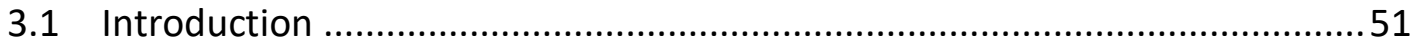

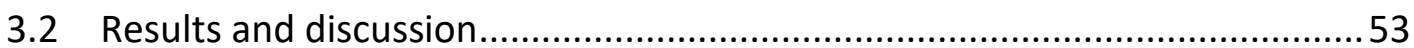

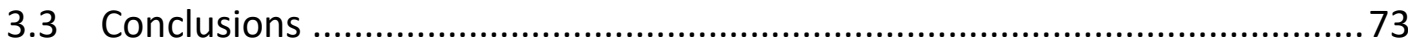

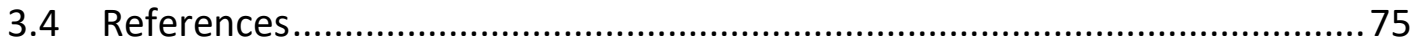

4 Catalyst-free one step synthesis of large area vertically stacked N-doped graphene-boron nitride heterostructures from biomass source ..........................79

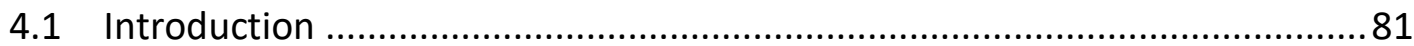

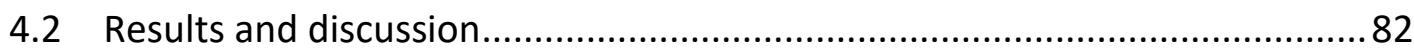

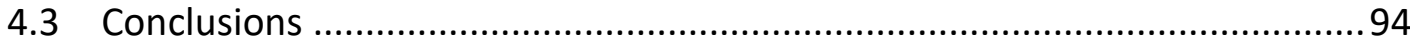

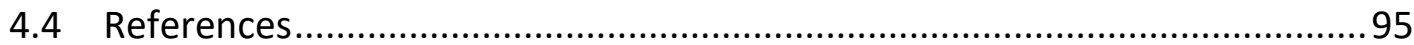

5 One-step preparation of large area films of oriented $\mathrm{MoS}_{2}$ nanoparticles on multilayer graphene and its electrocatalytic activity for hydrogen evolution ......103

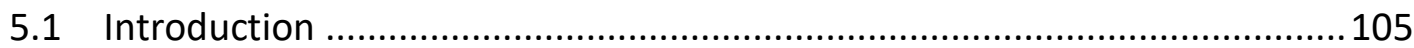

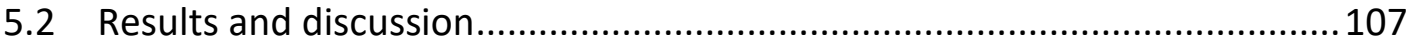

5.2.1 Sample preparation and characterization .............................................107

5.2.2 Electrocatalytic measurements ...............................................................116

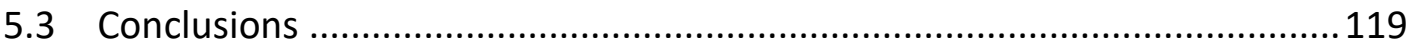


6 Iron nanoparticles embedded in graphitic carbon matrix as heterogeneous catalysts for the oxidative $\mathrm{C}-\mathrm{N}$ coupling of aromatic $\mathrm{N}-\mathrm{H}$ compounds and amides

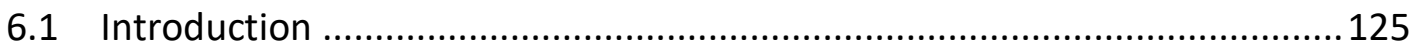

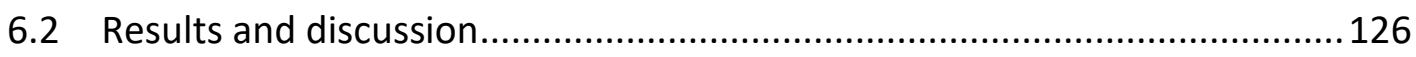

6.2.1 Catalyst preparation and characterization ..........................................126

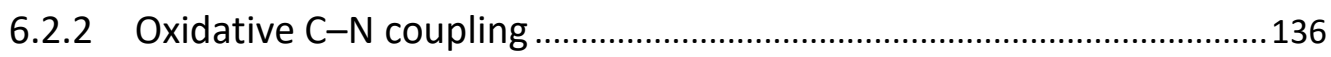

6.2.3 Reusability and catalyst stability ........................................................ 141

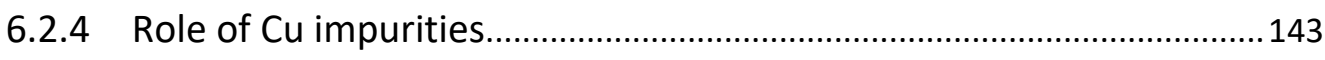

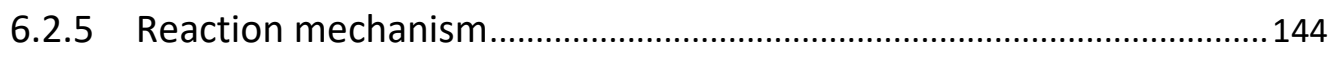

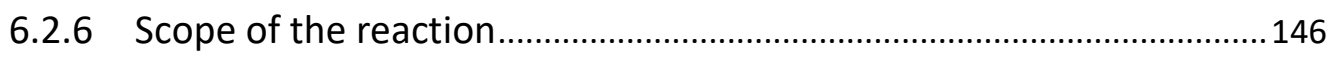

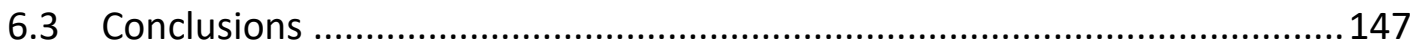

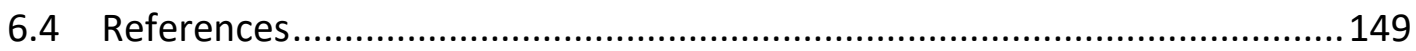

7 Extremely high selectivity towards isobutane in $\mathrm{CO}_{2}$ hydrogenation catalyzed by Fe-Co alloy nanoparticles embedded in graphitic carbon matrix....................155

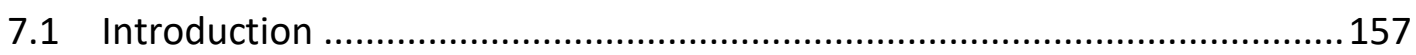

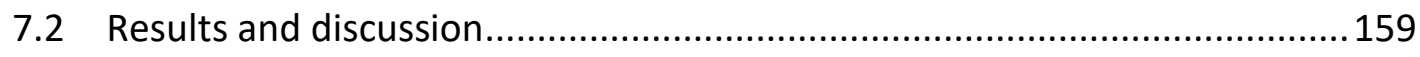

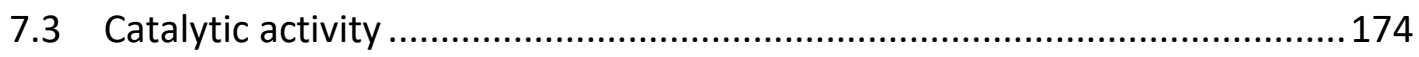

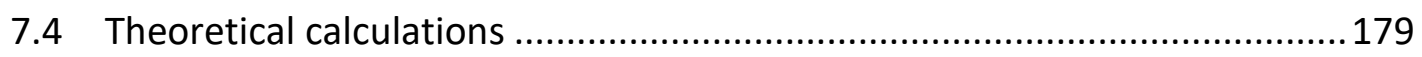

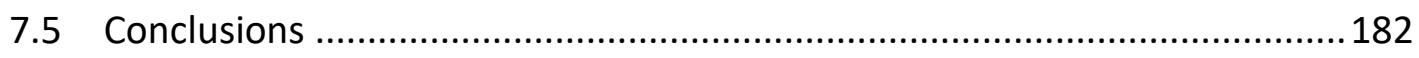

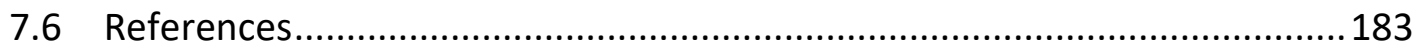

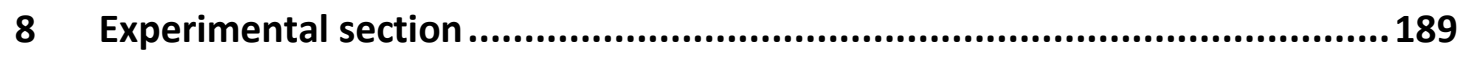

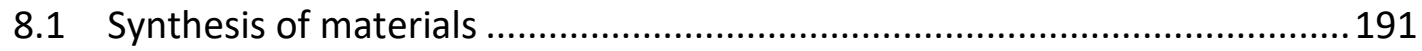

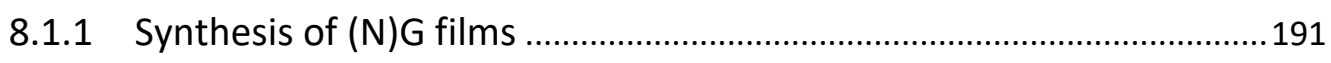

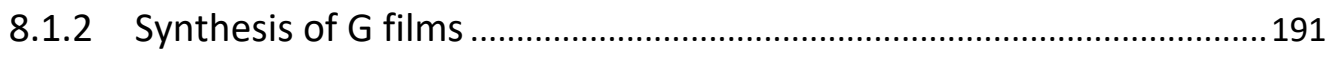

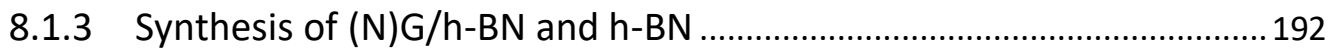

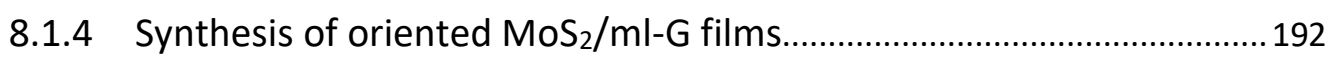

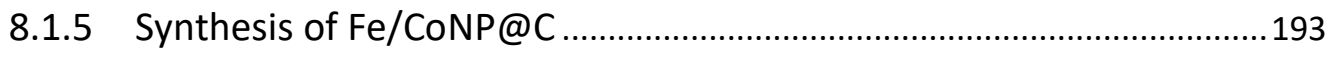




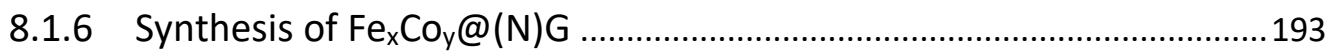

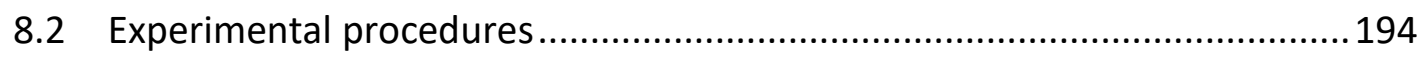

8.2.1 Photoelectronic tests for (N)G and G films used as electrodes...........194

8.2.2 Electrical measurements for (N)G/h-BN and (N)G ............................194

8.2.3 Electrochemical measurements for oriented $\mathrm{MoS}_{2} / \mathrm{ml}-\mathrm{G}$ films ..........195

8.2.4 General procedure for the oxidative C-N coupling ................................195

8.2.5 Reuse experiment for the oxidative $\mathrm{C}-\mathrm{N}$ coupling.................................196

8.2.6 Catalytic test procedure for the $\mathrm{CO}_{2}$ hydrogenation.............................196

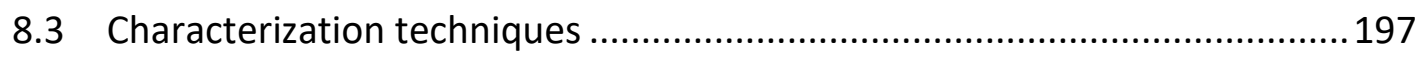

8.3.1 Transmission electron microscopy (TEM) ….........................................197

8.3.2 Scanning electron microscopy (SEM) …............................................... 197

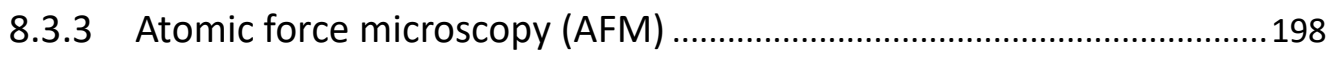

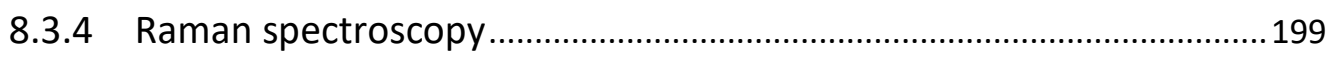

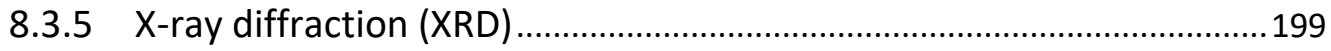

8.3.6 X-ray photoelectron spectroscopy (XPS) …….......................................200

8.3.7 Combustion elemental analysis.............................................................201

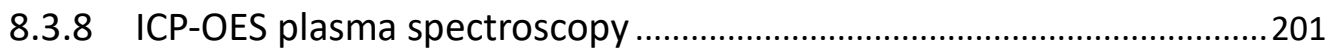

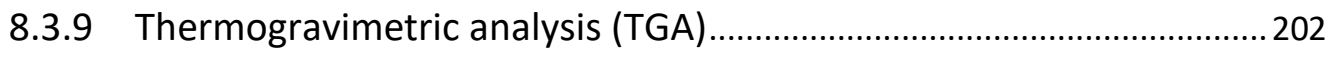

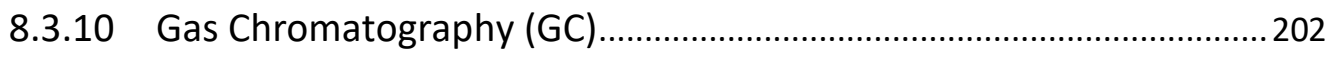

8.3.11 Gas Chromatography coupled to mass spectrometry (GC-MS) ........203

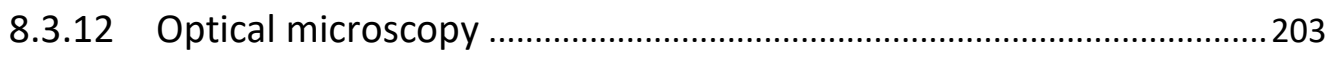

8.3.13 Brunauer-Emmett-Teller (BET) surface area analysis........................203

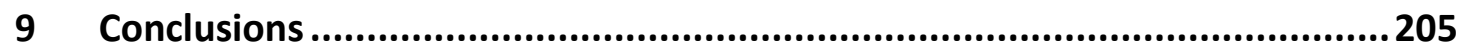

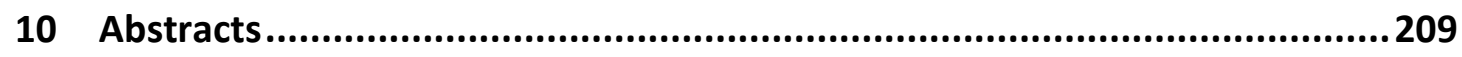

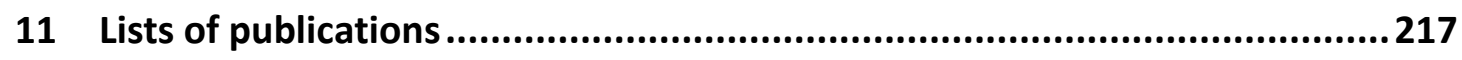



Chapter 1

General introduction

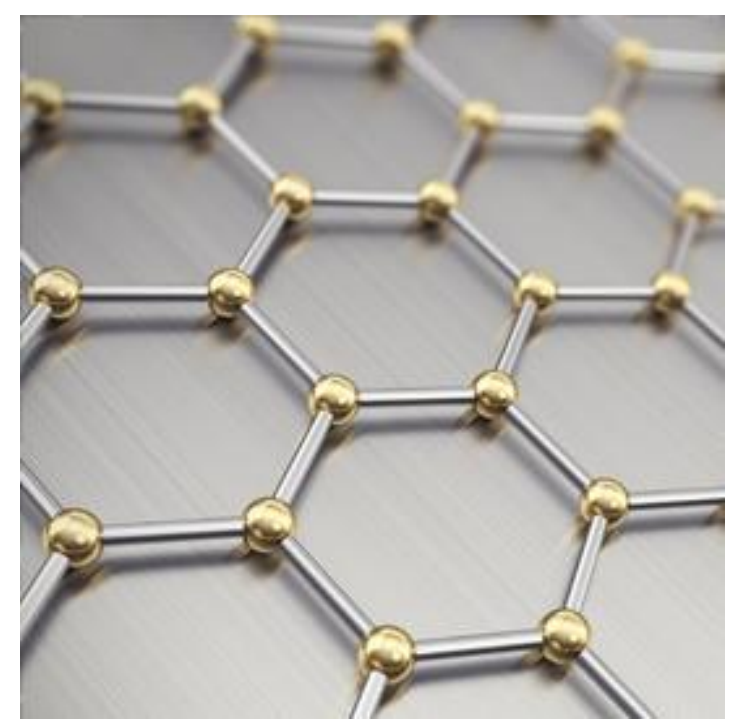





\subsection{Graphene}

Since the Nobel Prize for Physics was awarded in 2010 to Andre Geim and Konstantin Novoselov there has been a continuous and increasing interest in exploring and exploiting the unique properties of graphene. ${ }^{[1-3]}$ Although the existence of graphene was known since the earlier years of the XXst Century with the development of X-ray by Laue and Bragg ${ }^{[4]}$, isolation of a single graphene layer was not reported.

Due to its simple crystal structure and its high crystallinitity, pyrolytic graphite was one of the favorite materials to study the applicability of X-ray diffraction to determine the structure of crystalline solids. ${ }^{[5,6]}$ At that time it was determined that pyrolytic graphite was constituted by the stacking of layers, termed as graphene.

Graphene is a bidimensional (2D) material constituted by layers of a single atom depth constituted by carbon atoms with $\mathrm{sp}^{2}$ orbital hybridation in a hexagonal arrangement. Figure 1.1 shows a layer of ideal graphene and the crystal structure of graphite.

a

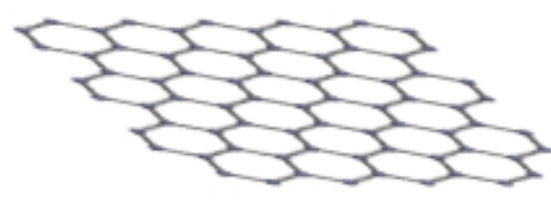

b

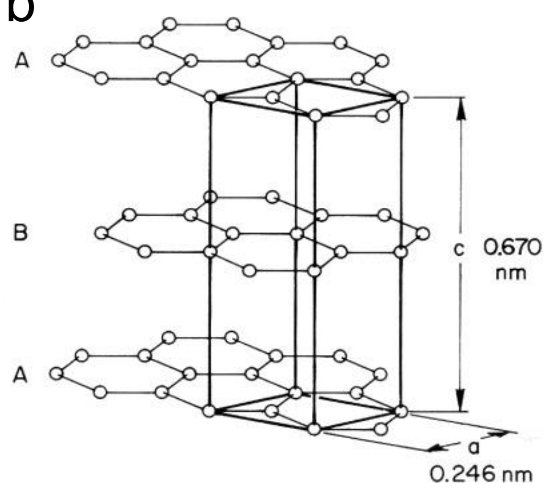

Figure 1.1. The honeycomb lattice of a layer of ideal graphene (a) and the structure of graphite (b).

However, although the existence of graphene was known, Geim and Novoselov were able to isolate for the first time a single monolayer and study their unique mechanical and electrical conductivity. The Swedish Academy of Science awarded the 
Nobel Prize to these researchers "for groundbreaking experiments regarding the two-dimensional material graphene". Being one atom thick graphene constitutes the physical limit of thickness for a material in where all the constitutive atoms are exposed to the external surface for interaction with the environment. A consequence is the large theoretical specific surface area of graphene that has been estimated as large as $2650 \mathrm{~m}^{2} \times \mathrm{g}^{-1} \cdot{ }^{[7,8]}$

The fact that all the atoms are accessible is also responsible for the large adsorption capacity of graphenes. Since the electronic configuration of graphenes has highest occupied molecular orbitals constituted by an extended $\pi$ cloud above and below the basal graphene plane, graphenes can interact with adsorbates by relatively strong $\pi-\pi$ interactions, as well as dipolar interactions and van der Waals forces. ${ }^{[9-12]}$ Figure 1.2 shows the possible interactions between graphenes and adsorbates.

a

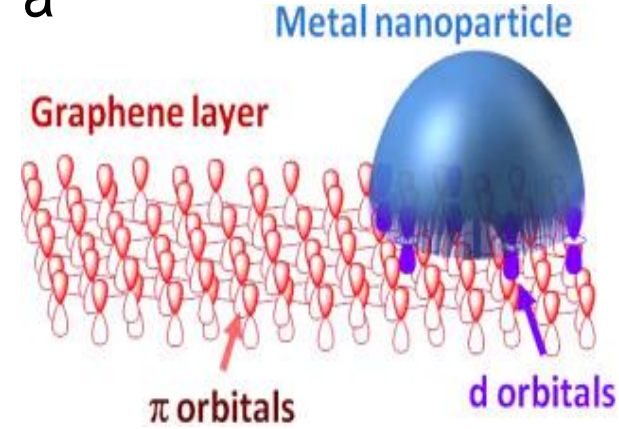

b

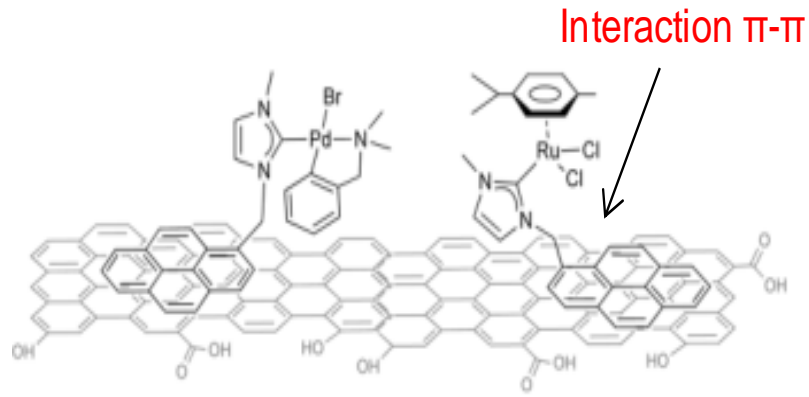

Figure 1.2. (a) Interaction of the $\pi$ orbitals of $G$ with the atomic orbitals $d$ of metal $\mathrm{NPs}^{[13]}$ and (b) Non-covalent intermolecular $\pi-\pi$ interaction of $\mathrm{G}$ with other flat molecules (pyrene) bound to metal complexes (Ru, Pd). ${ }^{[14,15]}$

These $\pi-\pi$ interactions are also responsible for the tendency of graphenes to undergo self-stacking and agglomeration. According to the Carbon guidelines for graphene and related materials, the term graphene should be reserved for single layer materials, something that requires the material to be deposited as film on a surface of a substrate or to be suspended in a liquid medium. As solid powders, some 
percentage of aggregation occurs naturally as can be determined by the appearance in these powders of broad diffraction peaks, indicating some degree of stacking diffracting X-rays.

Besides ideal graphene constituted exclusively by carbons in hexagonal geometry, there are defective graphenes in where pentagons, heptagons, carbon vacancies and even holes can be present on the layer. ${ }^{[16-20]}$ Other possible defects are related to the presence of heteroatoms in the structure, either in low proportion ("dopants") or in larger proportion. Among these heteroatoms oxygen and nitrogen are the most common ones, but there have been reports indicating the presence on the defective graphene of other heteroatoms, including $\mathrm{B}, \mathrm{S}, \mathrm{Si}$, halogens, etc. ${ }^{[21-25]}$ Some of them can be the possible active sites on the graphene sheets for the catalytic reactions. Figure 1.3 summarizes possible defects that can be encountered on the structure of defective graphenes.

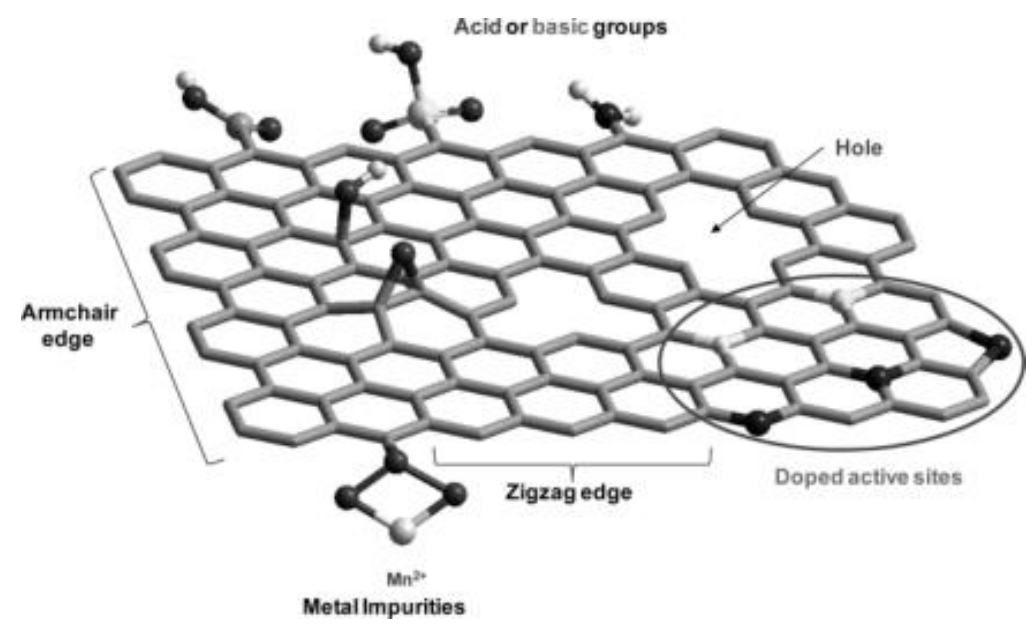

Figure 1.3. Representation of the types of defects present in a sheet of graphene. ${ }^{[26]}$

The presence of defects modifies the intrinsic properties of ideal graphene. In particular, it has been reported that under favorable conditions ideal graphene behaves as a zero-band gap semiconductor enjoying high electrically conductivity, comparable to that of $\mathrm{Cu}$ and $\mathrm{Ag}$. In addition, electron mobility in graphene is even larger than in metals and, for this reason, graphene is an ideal material for 
optoelectronics, where fast response of the material at $\mathrm{GHz}$ frequencies can be achieved. ${ }^{[1,2,27,28]}$ This fast response has implications in areas such as transmission of information in which larger amount of data can be submitted in shorter time. The presence of defects may cause the creation of a band gap with separation of the conduction and valence bands in energies that can be proportional to the density of defects on the graphene layer. ${ }^{[29-32]}$ Also, electrical conductivity decreases substantially from the maximum value and electron mobility becomes slower. Figure 1.4 illustrates the change from conductive to semiconductive graphene, as consequence of the presence of defects and heteroatoms on the graphene layer.

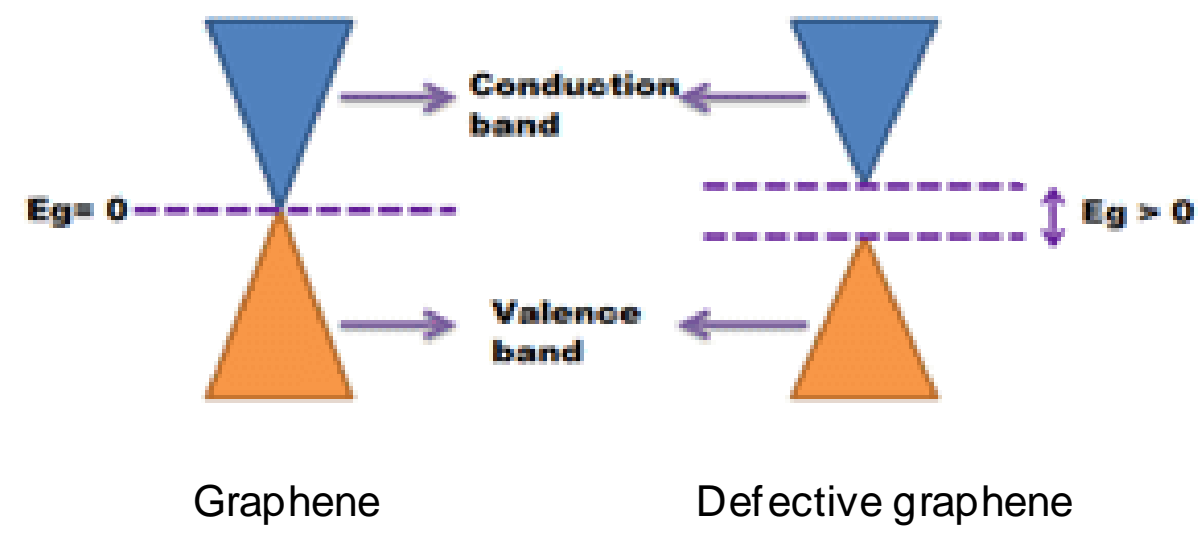

Figure 1.4. Band gap of pristine graphene and defective graphene. ${ }^{[33]}$

This change from conductive to semiconductive graphene is also reflected on the optical absorption and transparency of graphene. In principle, ideal graphene has a negligible absorption for radiation wavelengths in the whole range from UV to the visible. Stacking of graphene layers increases light absorption, but the material still has a "neutral" absorption in the UV-vis region, meaning that absorptivity and transparency is constant at all wavelengths. Stacking of four layers can be visually detectable by a gray tint that tends to black and reflective surface upon increasing the number of layers. ${ }^{[34,35]}$ Figure 1.5 shows transmittance properties of single and few-layers graphene films. As it can be seen, the single lay graphene can have a value of about $97 \%$ in optical transmittance. 
a

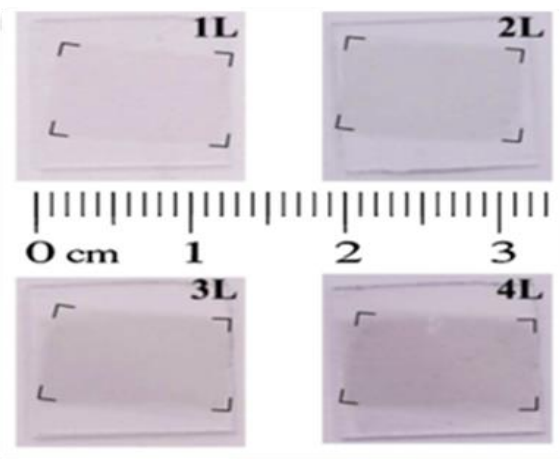

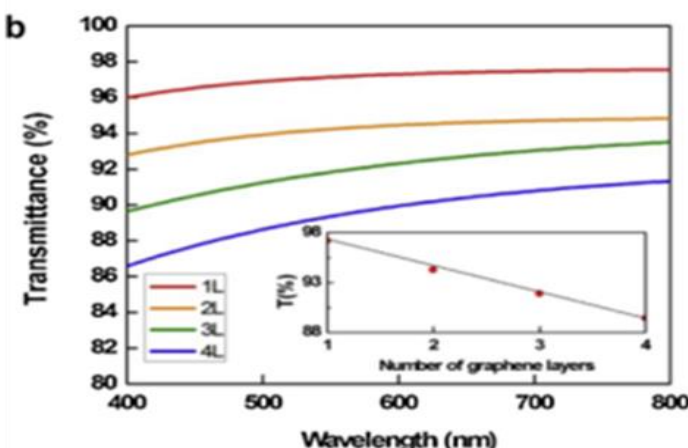

Figure 1.5. (a) Photographs of graphene films with 1-4 layers after the transfer produce to the glass substrate and (b) optical transmittance of these single and few-layers graphene films. The inset shows the transmittance of stacked graphene at $\lambda=550 \mathrm{~nm}$ as a function of the number of layers. ${ }^{[36]}$

The presence of defects determines the appearance of an absorption band, generally in the UV region that is associated to electronic transitions from the valence to the conduction band of the semiconductor. As typical inorganic semiconductors, defective graphenes can classified as " $p$ " or " $n$ " semiconductors depending on the Hall effect in which electrical continuous currents undergo left or right deviation in the presence of intense magnetic fields. This is related to charge mobility and is due to the nature of the charge carrier, either electrons or holes. Figure 1.6 illustrates the Hall effect in a conductor.

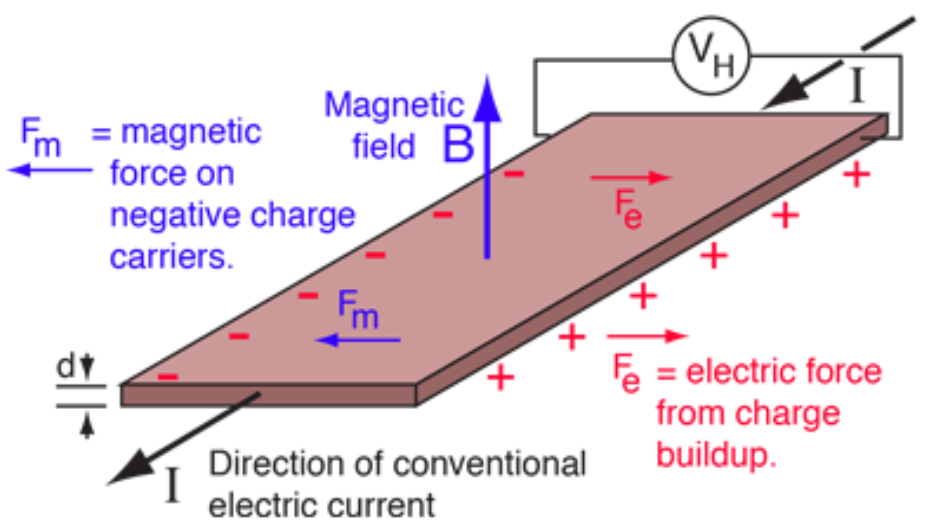

Figure 1.6. Representation of the Hall effect produced by the separation of charges. A current flows through the interior of conductor, in the presence of a magnetic field with a component perpendicular to the movement of the charges. 
Figure 1.7 summarizes many other unique properties of graphenes that, as commented earlier, would depend on the presence of defects, doping and the degree of stacking.

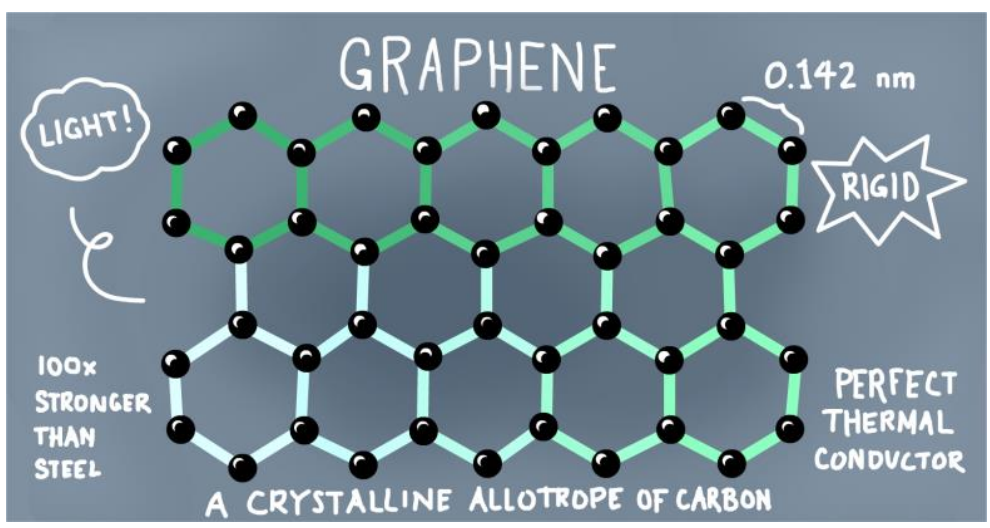

Figure 1.7. Illustration of the structure of graphene and some of its main properties.

\subsection{Graphene preparation}

There are basically two strategies for graphene preparation, denoted as "bottom-up" and "top-down". Figure 1.8 summarizes these two complementary approaches for graphene formation.

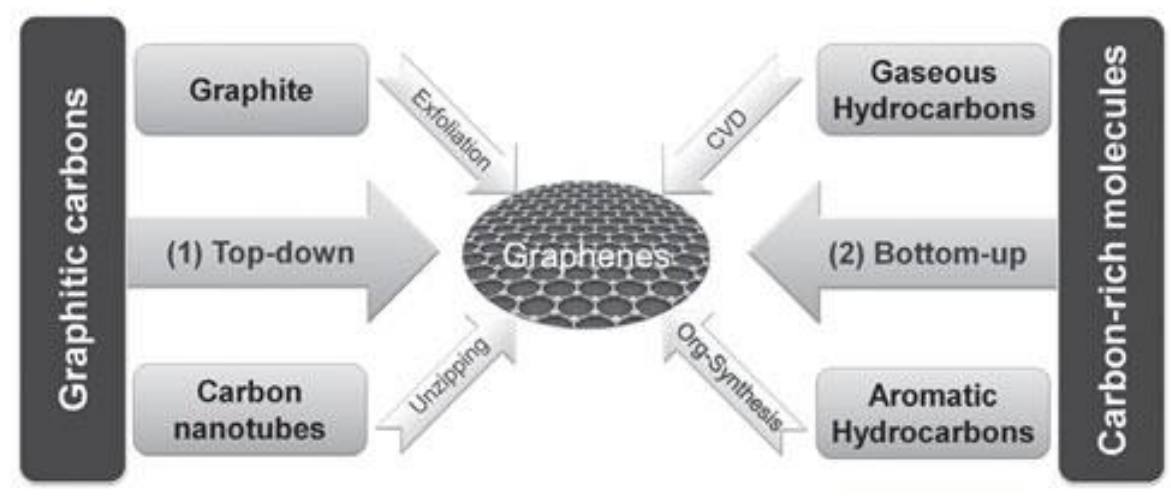

Figure 1.8. Two general strategies, top-down and bottom-up, for the preparation of graphene. 
In the bottom-up approaches small molecules condense to form the graphene sheet. The most widely used method is chemical vapor deposition (CVD) synthesis on hot metal surfaces. ${ }^{[37-41]}$ The typical precursor in this CVD process is methane and the atmosphere contains some hydrogen also. The metals most widely used in the CVD method are $\mathrm{Ni}$ and $\mathrm{Cu}$ that are heated at temperatures about $1000{ }^{\circ} \mathrm{C}$. These metals, at the reaction temperature have catalytic hydrogenating/dehydrogenating activity and cause decomposition of methane into carbon and hydrogen, a process that can have even interest as a way to obtain hydrogen gas from methane. The process starts with the cleaning of the metal surface by exposing it to hydrogen at the reaction temperature. This step cleans the possible oxide overlayer that could be present on the $\mathrm{Ni}$ or $\mathrm{Cu}$ sheet, forming water in the process. After cleaning the metal surface, the chamber is fed with methane that undergoes decomposition. Equation 1.1 summarizes the reaction taking place, while Scheme 1.1 illustrates the CVD process of graphene formation and Scheme 1.2 describes the mechanism of the process.

$$
\mathrm{CH}_{4}(\mathrm{~g}) \rightarrow \mathrm{C}_{\mathrm{g}}+2 \mathrm{H}_{2}(\mathrm{~g})
$$

Equation 1.1. The equation for describing the graphene growth in a reactive $\mathrm{CH}_{4} / \mathrm{H}_{2}$ atmosphere in the CVD process. ${ }^{[42]}$

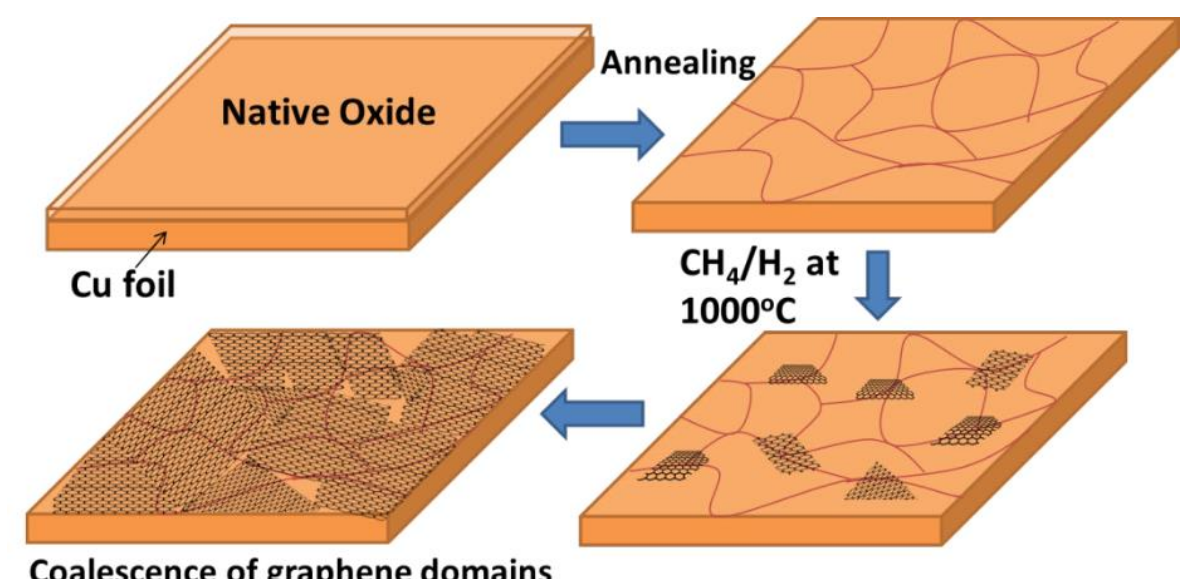

Scheme 1.1. Procedure for the formation of graphene using the CVD technique. ${ }^{[43]}$ 


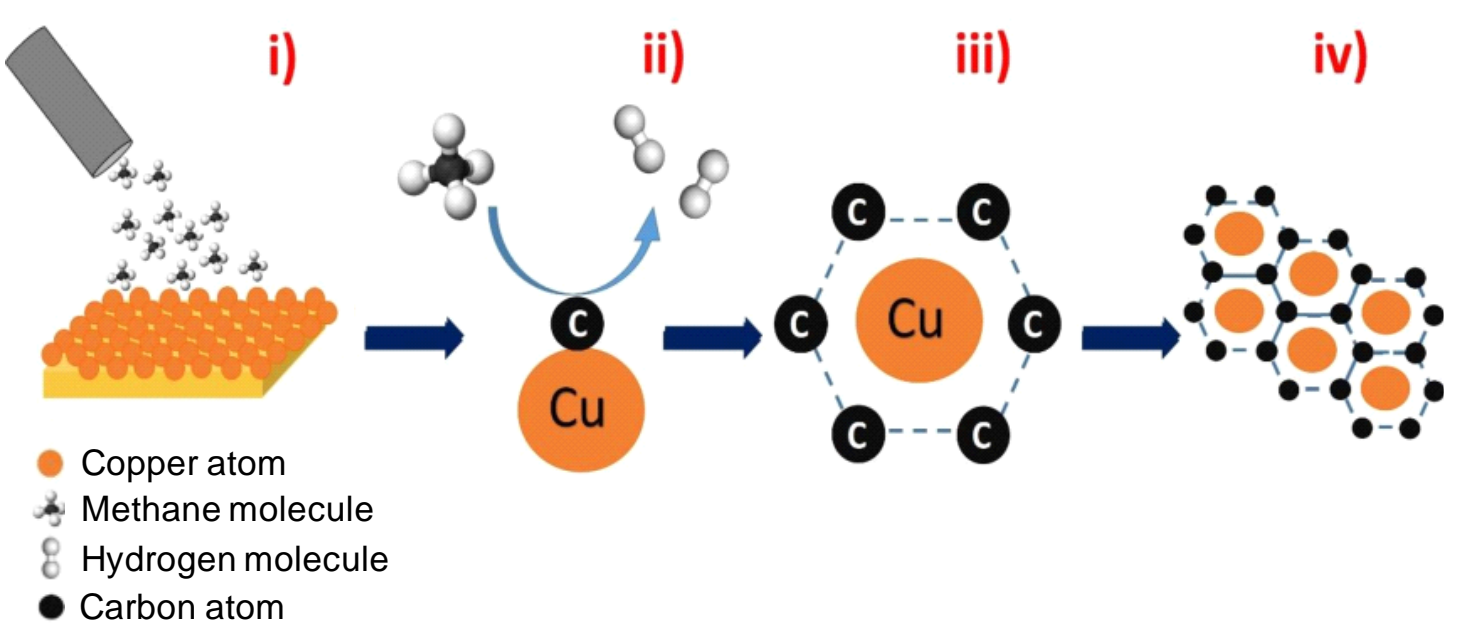

Scheme 1.2. Description of the mechanism of formation of a graphene film on a copper foil by the CVD process in several stages: (i) The copper foil is heated under vacuum to $1500{ }^{\circ} \mathrm{C}$ and a hydrocarbon such as methane is dosed. (ii) The hydrocarbon gets in contact with the copper atom and is pyrolyzed, resulting in the formation of hydrogen and atomic carbon that is deposited on the copper atom. (iii) Perpendicular view of these carbon atoms that are ordered on the copper atom in hexagonal form due to the template effect exerted by the copper atom. (iv) Finally on each copper atom the same thing happens, generating a carbon film in all the copper atoms and over time these carbon atoms spontaneously bond and generate a sheet of graphene on the "sea" of copper atoms.

As it can be seen in Schemes 1.1 and 1.2, as the $C$ atoms from methane deposit, they remain on the surface due to the low solubility of $\mathrm{C}$ in $\mathrm{Cu}$ or at the surface and subsurface in the case of $\mathrm{Ni}$, for which $\mathrm{C}$ solubility is somewhat higher than for $\mathrm{Cu}$. These carbon atoms at the metal surface start to surround each of the $\mathrm{Cu}$ or $\mathrm{Ni}$ atoms of the surface forming hexagons. Up to six $\mathrm{C}$ atoms can crown a $\mathrm{Ni}$ or $\mathrm{Cu}$ atom and this is one of the reasons why the graphene sheet develops in the CVD method. This mechanism of graphene formation by CVD has been supported by the matching of the 111 surface of $\mathrm{Cu}$ and $\mathrm{Ni}$ with the graphene sheet. Therefore, if other different surfaces of the metals are exposed in the CVD process, the graphene growth 
becomes considerably less favorable due to lattice mismatch and also the sheet has less quality due to the presence of defects that include the formation of pentagonal and heptagonal cycles and carbon vacancies in addition to the prevalent hexagonal geometry of ideal graphene. For these reasons, high quality graphene sheets are prepared with $\mathrm{Cu}$ and $\mathrm{Ni}$ metal surface with exposing 111 planes. The CVD process was patented by Samsung and subsequent patents refer at how to transfer the graphene sheet from the metal surface to other arbitrary surfaces. In fact, the main limitations of the CVD method are the need of $\mathrm{Cu}$ or $\mathrm{Ni} 111$ surface and the long time required to prepare high quality single-layer graphene. Fast graphene growth is also prone to produce defects and even multilayers graphene patches not uniformly distributed combined with graphene holes.

Besides the CVD method, the group of Mullen has been actively developing graphene preparation procedures based on concepts of organic synthesis. In these processes an aromatic precursor undergoes polycyclizations forming additional rings. In this way, graphenic sheets having relatively small sizes, barely reaching the micrometric size can be obtained. Figure 1.9 shows some examples of the precursors and the organic synthesis strategy followed to form graphenic sheets.

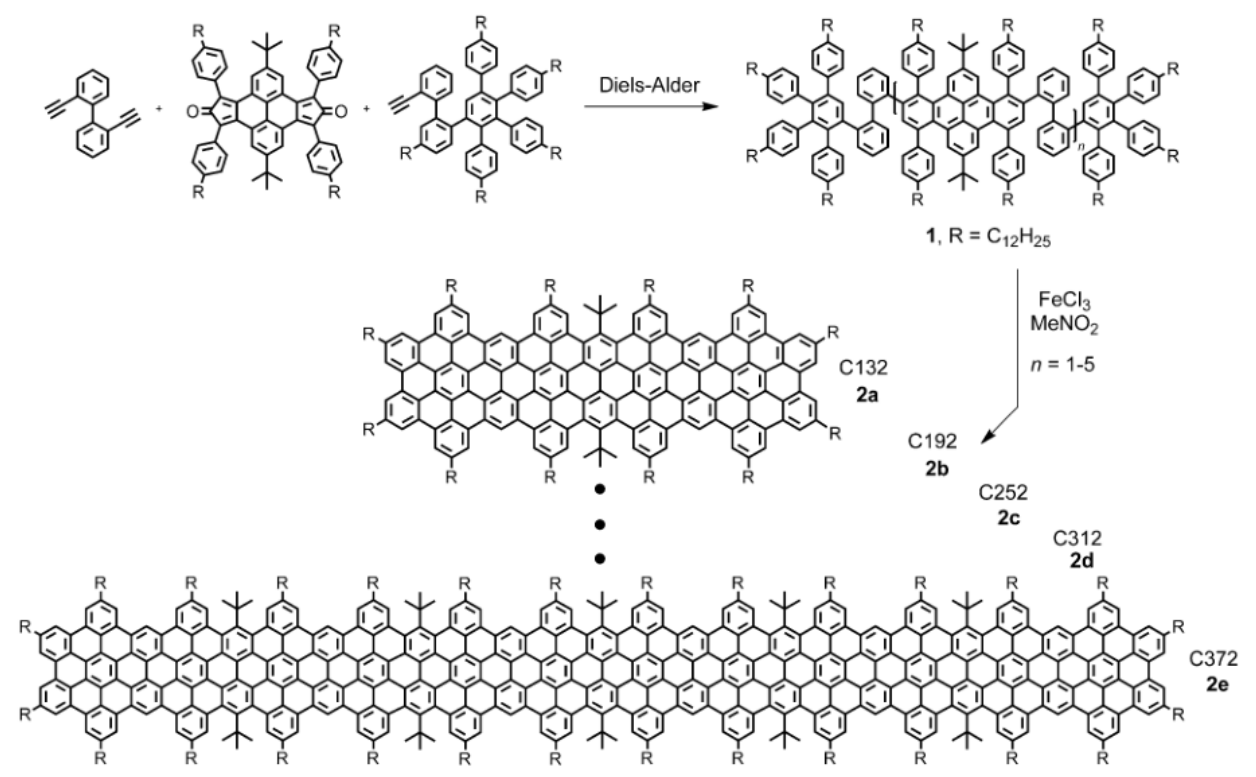

Figure 1.9. A bottom-up approach for the preparation of $\mathrm{G}$ material based on organic

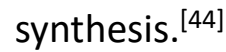


Although this approach is very promising to prepare graphenes with desirable substructures and can be useful to obtain special graphenes, there are still some disadvantages related to the availability of precursors, the need of noble metal catalysts to promote the polycyclization and the occurrence of defects associated to incomplete cyclications and the presence of residual initial functional groups. As it will be commented later, one of the problems related with applications of graphene in certain areas, like metal-free catalysis, is the possible presence of metals even in trace quantities that could impurify the sample. There is sometimes the possibility that the observed catalytic activity derives from the presence of metals even in ppm or lower concentrations. For applications in microelectronics, also the presence of metals is highly detrimental for the electronic response of the materials. In general, to avoid the presence of defects, large quantities of metal catalysts are employed and they can be difficult to remove completely.

Besides bottom-up methodologies, the most widely employed approaches to obtain graphene are the top-down. Many of them, rely on the fact that graphite already contains graphene sheets. The problem is, however, how to separate isolated, individual graphene layers. Geim and Novoselov used manual mechanical graphene exfoliation of graphite (Figure 1.10).. ${ }^{[1,2]}$ While this process produces high quality graphene samples starting from high-crystallinity pyrolytic graphite, it can only be used to obtain a few samples, mostly for the purpose of physical properties measurements.

Direct exfoliation of graphite by ultrasonication in solvents requires the use of highly-viscous, low-volatility liquids that later are very difficult to separate from the graphene. In addition the yield of this process in terms of the mass of graphite that can be exfoliated is very low, frequently below $0.1 \%$. Typical solvents used in this direct exfoliation process are $\mathrm{N}, \mathrm{N}$-dimethylformamide (DMF), N-methylpirrolidone (NMP), dimethylsulfoxide (DMSO) and ionic liquids, among others. ${ }^{[45-49]}$ The presence of additives, mainly surfactants, polycyclic aromatic molecules can assist the exfoliation of graphite, but then, again, removal of the additive becomes very 
difficult.

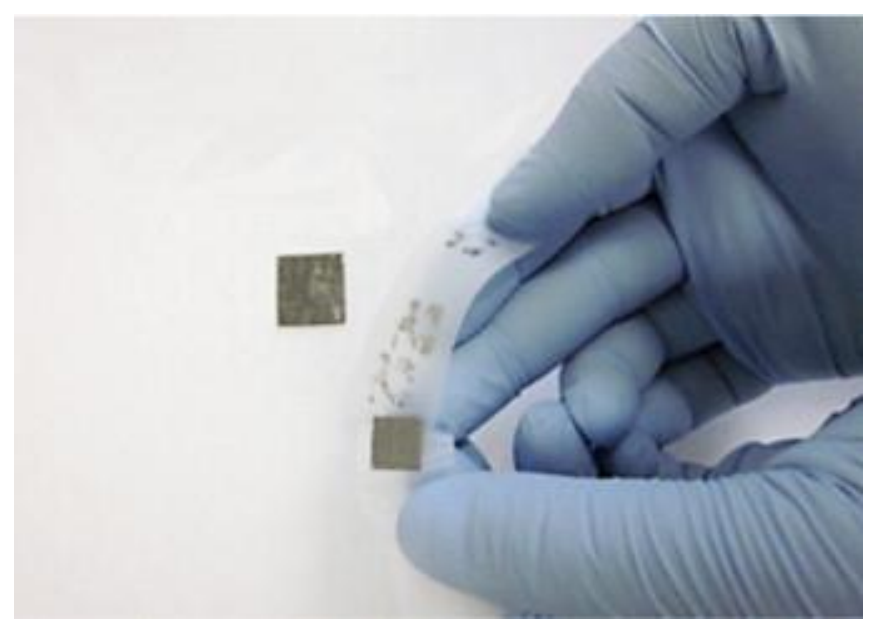

Figure 1.10. Graphene synthesis from HOPG block by using Scotch tape method.

For all the above problems, the most widely used method to obtain graphene materials in large quantities is based on the deep oxidation of graphite to form graphite oxide, followed by exfoliation and reduction. ${ }^{[50-52]}$ The process is presented in Figure 1.11. It was known that graphite can be deeply chemically oxidized to form graphite oxide using a series of chemical reagents. The most popular one is potassium permanganate in a highly concentrated mixture of nitric and sulfuric acids. This procedure was reported by Hummers and Offenbach in the mid fifties of the last century. ${ }^{[53]}$ In contrast to graphite, graphite oxide may contain up to $60 \mathrm{wt} . \%$ of oxygen and the sheets are constituted by graphene oxide. The oxygen atoms in graphene oxide are present as oxygenated functional groups, including epoxide, hydroxyls, carbonyls and carboxylic acids. Figure 1.12 presents a general structure of graphene oxide that is in agreement with analytical and spectroscopic data, particularly IR and ${ }^{13} \mathrm{C}$ NMR spectroscopies as reported by Klinowsski and co-workers. 


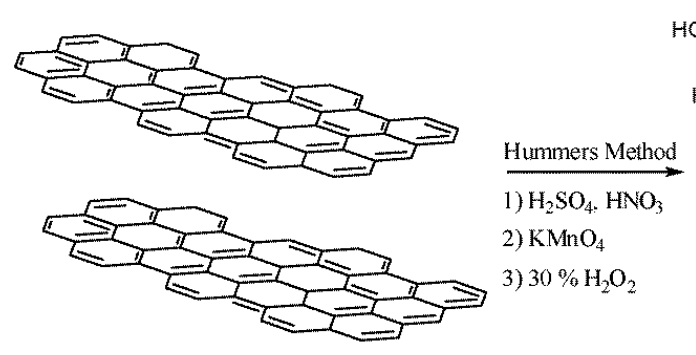

Graphite Powder

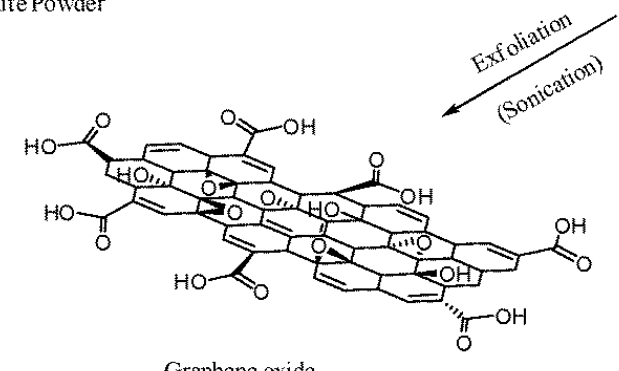

Graphene oxide

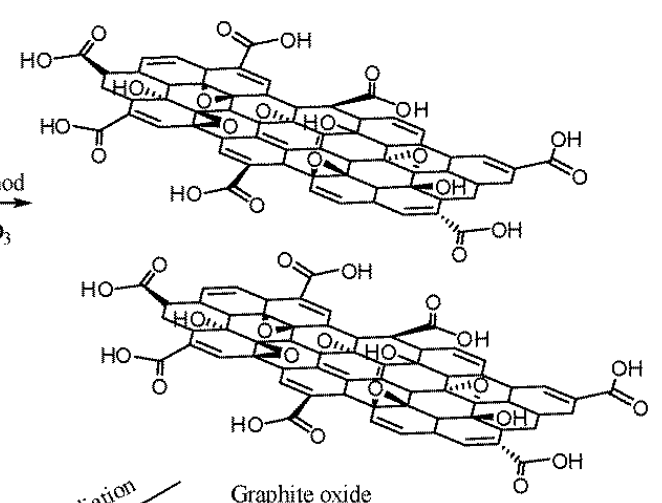

Graphite oxide "I $\mathrm{OHH}^{\mathrm{OH}}$

Figure 1.11. Procedure used for chemically assisted exfoliation of graphite through Hummers oxidation. ${ }^{[54]}$

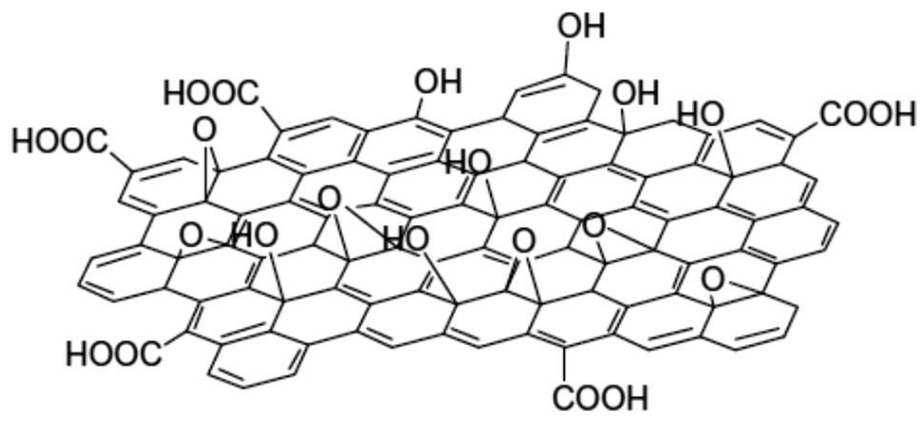

Figure 1.12. Proposed structure of graphene oxide. ${ }^{[55]}$

In contrast to graphite, graphite oxide can be easily exfoliated to the state of single, isolated layers of graphene oxide in essentially complete yields. The reason for this is the increase in the interplanar distance between the sheets due to the intercalation of oxygenated functional groups. While graphene sheets in graphite are separated by $0.34 \mathrm{~nm}$, the interlaminar distance in graphene oxide sheets in graphite oxide is $0.69 \mathrm{~nm}$ that is more than double. In addition, the strong $\pi-\pi$ interaction present in graphene that causes the high crystallinity of graphite does not exist in graphene oxide due to the lack of $\mathrm{C}=\mathrm{C}$ double bonds and aromatic regions. 
Furthermore, under certain $\mathrm{pH}$ ranges, the graphene oxide can exhibit repulsive interactions due to the high density of negatively charged carboxylate groups. Therefore, ultrasonication of graphite oxide in water produces the destruction of the solid and the appearance of a persistent, stable suspension of graphene oxide that is highly hydrophilic. Concentrations as high as $10 \mathrm{mg} \mathrm{mL}^{-1}$ of graphene oxide can be stable in aqueous phase.

After exfoliation, a large degree of reconstruction of graphene is possible either by chemical reduction of graphene oxide or by physical treatments. Among the most employed reducing chemicals that have been used to reduce graphene oxide, hydrazine and metal hydrides can promote at room temperature the partial conversion of graphene oxide into some kind of defective graphene that is generally termed as reduced graphene oxide. ${ }^{[56-59]}$ Figure 1.13 illustrates a proposed mechanism for chemical reduction by applying hydrazine as reducing reagent. The problem of this approach is that the sample of defective graphene becomes contaminated by the excess of the chemical agent that is generally difficult to separate from the reduced graphene oxide in aqueous suspension. Also some degree of $\mathrm{N}$ doping can be also promoted in the process.

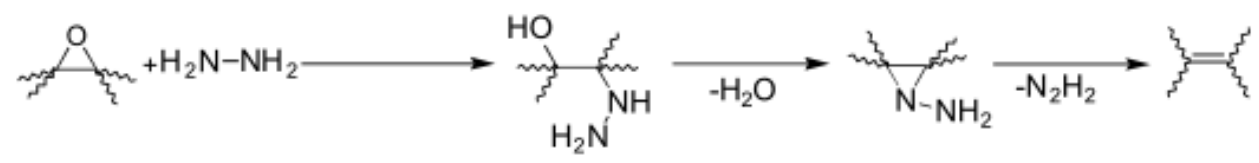

Figure 1.13. A possible reaction pathway for epoxide reduction using hydrazine as reducing reagent. ${ }^{[60]}$

Besides chemical reactions, graphene oxide can also be reduced to reduced graphene oxide by thermal treatments and hydrothermal processes. ${ }^{[61-66]}$ This is one of the procedures followed in the present Doctoral Thesis to obtain samples of defective reduced graphene oxide. It consists in the treatment of the aqueous suspension obtained in the sonication of graphite oxide in an autoclave at 
temperatures about $150{ }^{\circ} \mathrm{C}$ for several hours. Under these conditions evolution of gases, mainly $\mathrm{CO}_{2}$ from graphene oxide leads to an effective decrease in the oxygen content of the materials. Since reduced graphene oxide is considerably less hydrophilic than graphene oxide, then, dispersion of this material in aqueous phase can only be achieved for much more diluted samples, typically one order of magnitude more diluted, and spontaneous separation of reduced graphene oxide from the aqueous phase occurs during the process.

The reduced graphene oxide is far from ideal graphene because it still contains a certain amount of oxygen that can be as high as $20 \%$ from the initial content of graphene oxide that is above $50 \%$. Besides oxygenated functional groups, other types of defects are carbon atom vacancies generated by $\mathrm{CO}_{2}$ evolution and even holes on the sheet. For this reason, the material obtained through graphene oxide by reduction is termed as reduced graphene oxide. For certain applications related to the high conductivity of graphene, reduced graphene oxide is far from the behavior expected for ideal graphenes. However, for some other applications, particularly for the use of these materials in catalysis that will be the subject of Chapter 4 on the electrochemical properties of graphene-boron nitride assemblies and also related to Chapters 5 and 6 on the catalytic activity of Fe and Co nanoparticles embedded on graphene matrices, the presence of defects is highly appropriate leading the introduction of active sites or providing strong metal-support interaction for anchoring of metals.

Besides the above methods for preparation of graphenes, our group developed a novel procedure based on the pyrolysis of carbohydrate that because of its importance in the present PhD thesis will be described in the next section. 


\subsection{Defective graphenes by pyrolysis of biopolymers}

Carbohydrates owe their name to their well-known property to form carbon residues upon dehydration either by heating or chemical reagents. These chemical compounds are the most abundant components of biomass while cellulose and hemicellulose account for over $60 \%$ of the mass in plants. Besides cellulose, there are also many natural polysaccharides, three examples of these biopolymers being alginates and carrageens from algae and chitosans from insect and crustacean skins. Figure 1.14 shows the structures of these natural polymers.

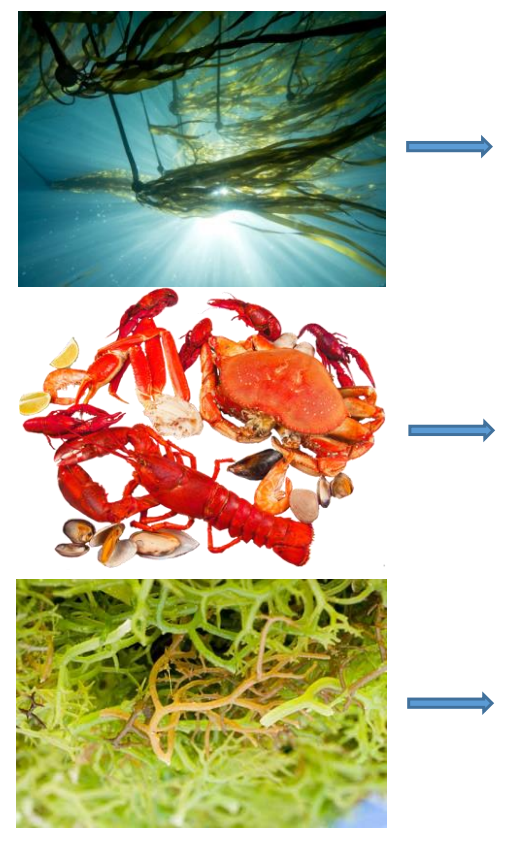

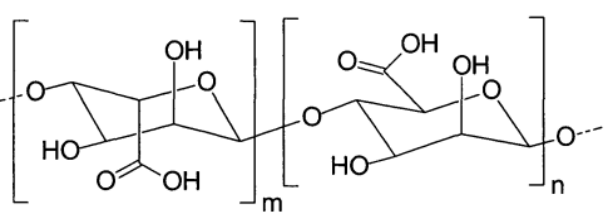

Alginate

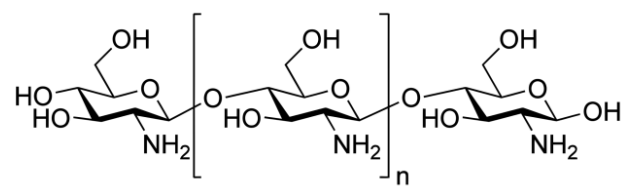

Chitosan

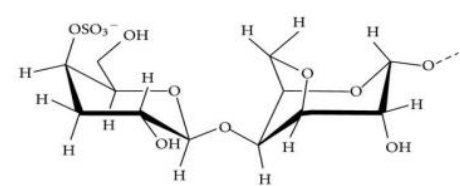

Carrageenan

Figure 1.14. Illustrations of sources of biomass (alginate, chitosan and carrageenan) and structures of the biopolymer precursors used to obtain graphene by our research group.

It was also known that upon pyrolysis, polysaccharides form carbon residues that were generally termed as "turbostratic graphitic carbons". [67-71] In fact, after pyrolysis of these polysaccharides an imperfect graphite as deemed by evaluation of the XRD patterns was formed. In XRD these turbostratic graphitic carbons exhibit a broad diffraction peak at $2 \theta$ angles somewhat shorter than $24^{\circ}$ that indicates that the 
material is not highly crystalline. This is indicated also by the term turbostratic that means that the stratus (graphene layers) are disordered (turbo). Figure 1.15 presents a XRD pattern of the carbon residue obtained upon pyrolysis of chitosan at $900{ }^{\circ} \mathrm{C}$ compared with that of well-crystalline graphite showing the remarkable differences regarding the shape and position of the characteristic diffraction peaks.

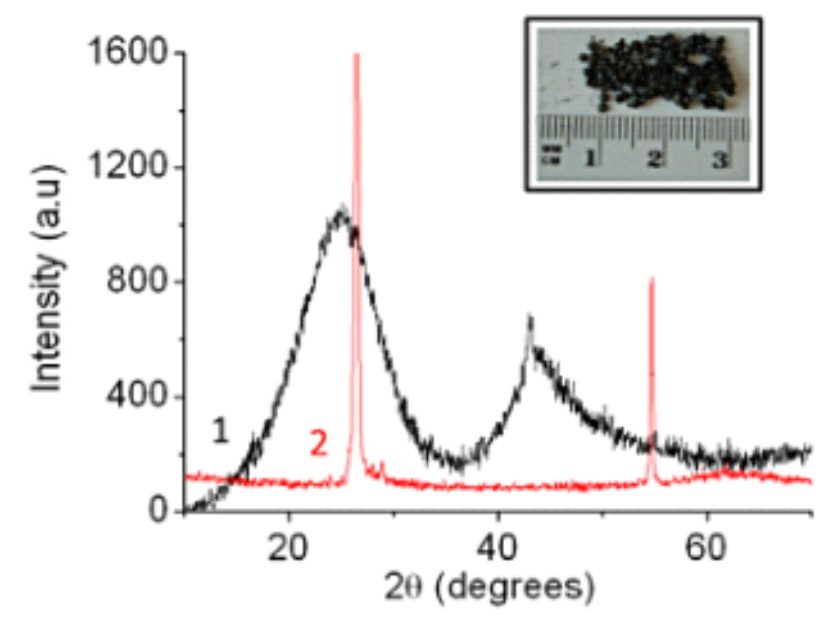

Figure 1.15. Graphene obtained by pyrolysis of chitosan obtaining signals that have very broad bands (1) and comparison with the XRD pattern of crystalline graphite that presents narrow and defined peaks (2). The inset shows photograph of the carbon residues. ${ }^{[72]}$

Although formation of turbostratic graphitic carbons in the pyrolysis of polysaccharides was known, our group was the first showing that sonication of this residue in aqueous medium or other solvents renders dispersions of defective graphenes with a yield over $70 \% .{ }^{[72-77]}$ The rest of the material could correspond to amorphous carbon that is not able to undergo exfoliation and dispersion. In some cases, the yield of defective graphene formation by sonication of the carbon residue derived from polysaccharide pyrolysis is close to be quantitative and, in any case much, much higher than that previously commented for the attempts of direct exfoliation of graphite.

The reasons for this facile exfoliation derive from the configuration of the 
graphitic carbon residue as being constituted by graphene layers that are loosely packed, in contrast to the case of graphite. This low crystallinity is reflected in the XRD with the broad diffraction peak, previously commented. Moreover, the fact that the peak appears at shorter angles indicates that the interlayer distance in the graphitic residue is much larger than in graphite and this decreases the strength of the interaction among the layers and, consequently, favors exfoliation.

The morphology of the turbostratic graphitic carbon residue obtained in the pyrolysis of polysaccharides can be seen by microscopy. Figure 1.16 shows a series of images of the turbostratic graphitic residue obtained from pyrolysis of chitosan at different magnifications.
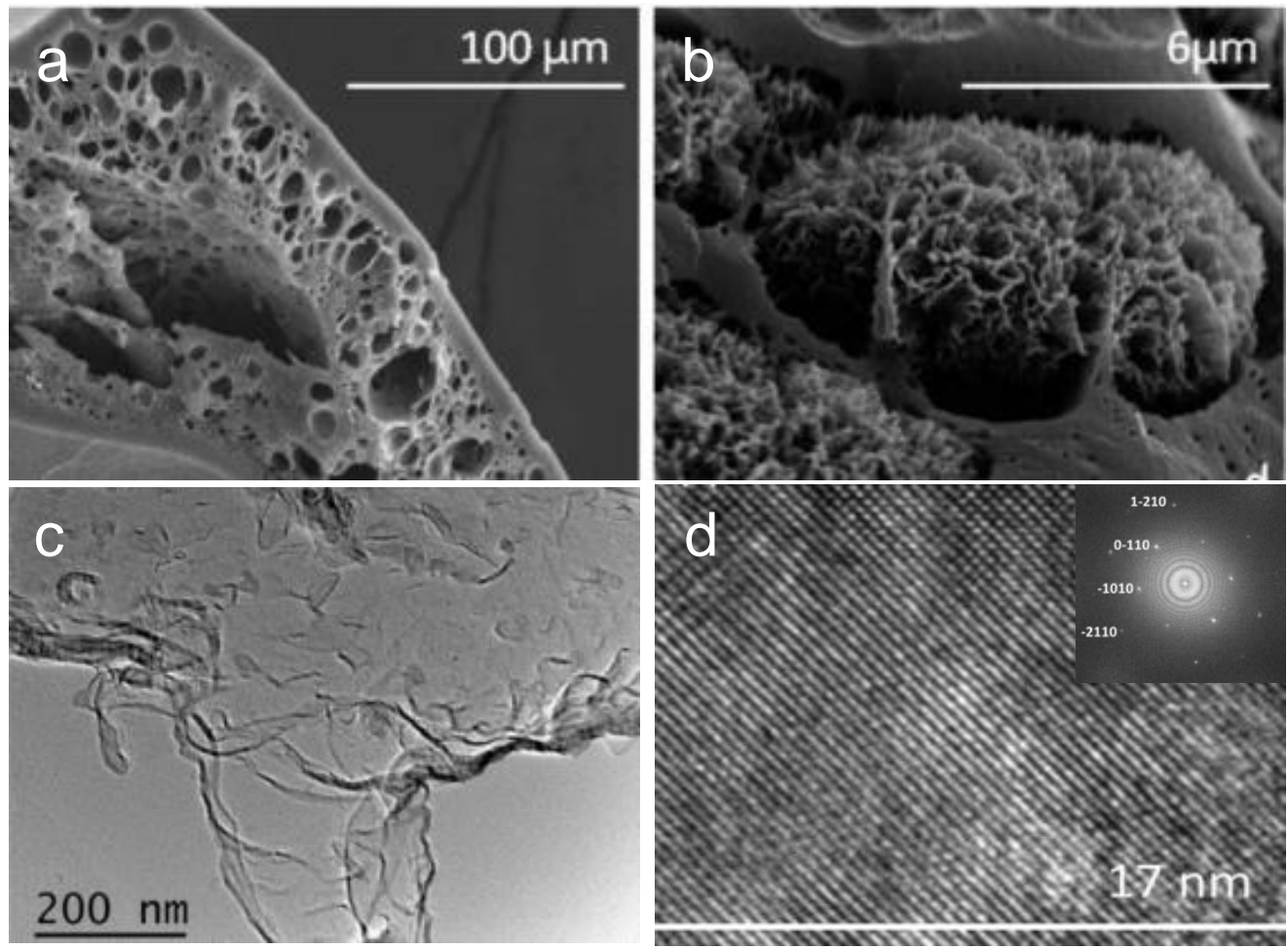

Figure 1.16. SEM images $(\mathbf{a}, \mathbf{b})$ of the carbonaceous residues obtained after the pyrolysis of natural polysaccharides. TEM images $(\mathbf{c}, \mathbf{d})$ of the resulting graphenes after the exfoliation of the carbonaceous residues by ultrasonic treatment. The inset shows the selected area electron diffraction (SAED) pattern of image d. ${ }^{[72,74]}$

Images from scanning electron microscope (SEM) of one of these samples 
formed as spherical beads shows that the spheres are hollowed and that the walls of present layers not properly packed, particularly in the inner part. The upper and lower parts of the walls tend to become more packed than the central parts. Higher magnification by transmission electron microscopy (TEM) shows that these layers of the walls break in the sonication process into the typical morphology of single layer graphene with the characteristic light color contrast and the presence of wrinkles corresponding to flexible sheets. High resolution transmission electron microscopy at quasi atomic resolution shows the crystalline domains of the sheets, while electron diffraction exhibits the expected hexagonal arrangement of the atoms in the material.

The single layer or few-layer morphology of the sheets can also be determined by atomic force microscopy (AFM) upon deposition on an atomically flat surface a drop of a dispersion of the defective graphene obtained by sonication of the turbostratic graphitic carbon residue formed in the pyrolysis. Typical substrates in this type of measurements are silica wafers or exfoliated mica materials. Figure 1.17 presents some measurements of the samples related to the present Doctoral Thesis. As it can be seen in this Figure 1.17, the frontal view shows that the defective graphene particles have a lateral size in the range of micrometers. By measuring the vertical height with subnanometric resolution, it can be determined that most of the defective graphene particles are constituted by a single or few layers. It is also assumed that the thickness of the monolayer of the defective graphenes prepared by pyrolysis of polysaccharides with a high residual oxygen content can be close to 0.4 $\mathrm{nm}$.

The presence of defects can be clearly determined by combustion chemical analysis, Raman spectroscopy and X-ray photoelectron spectroscopy (XPS). In combustion elemental analysis the presence of elements such as $\mathrm{N}$ and $\mathrm{S}$ can be directly quantified, together with the carbon content and it can be inferred that the difference to $100 \%$ should mainly correspond to residual oxygen. 


\section{Chapter 1}
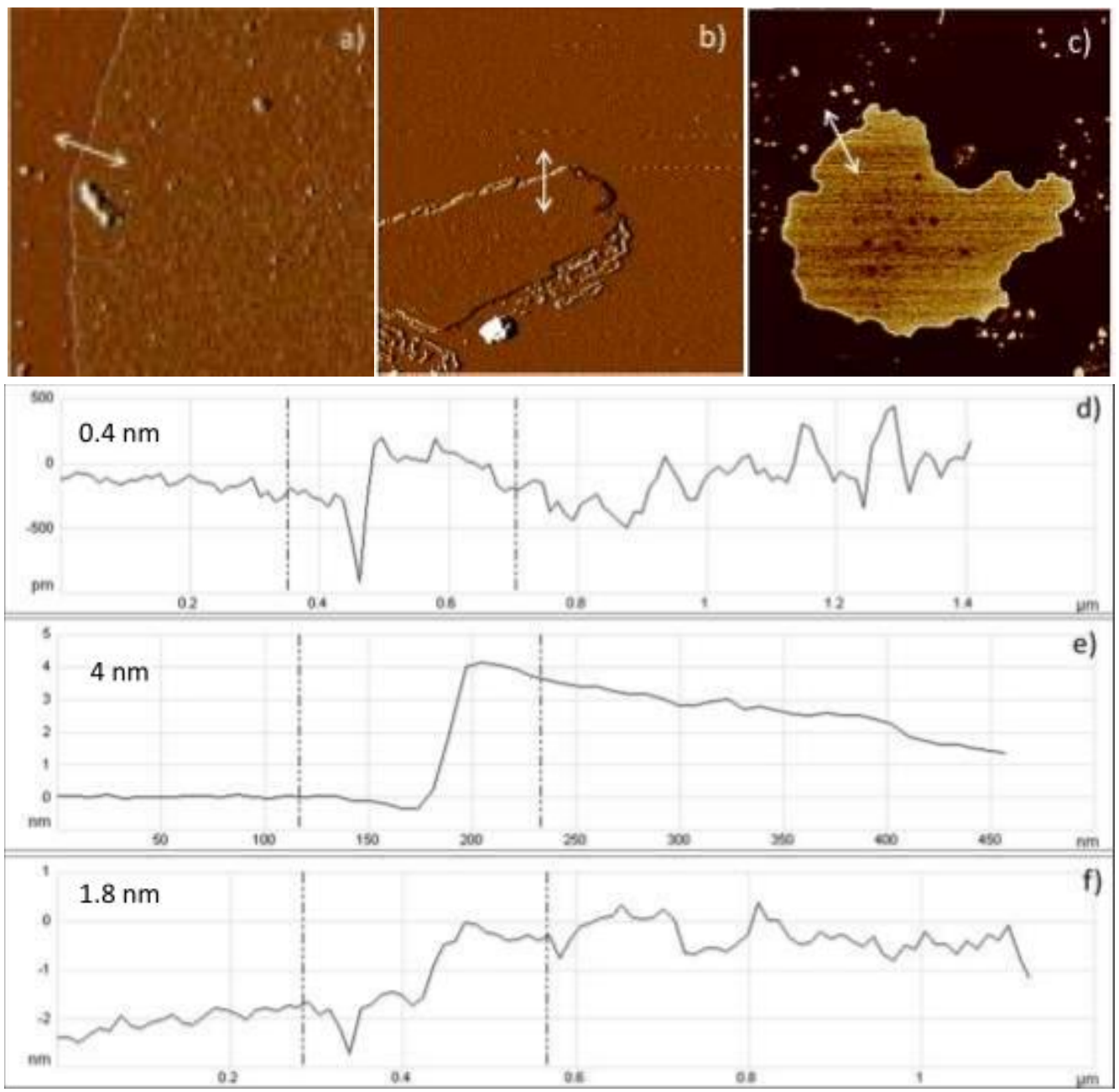

Figure 1.17. AFM images $(\mathbf{a}, \mathbf{b}, \mathbf{c})$ of the resulting samples and measurements of layer thickness where we can see the presence of a single sheet (d) or the stacking of several of them (e and $\mathbf{f})$.

The elements present on the surface of the material and their atomic ratio can be quantitatively determined by XPS. Since XPS is a surface technique and graphene and defective graphenes are 2D materials, XPS should in principle provide elemental compositions close to those measured by combustion chemical analysis. However, since most of the XPS measurements are performed on powders, rather than on films, where stacking should unavoidably occur, the values of XPS refer to the analytical composition of the outermost external surface that can be somewhat different from that of the bulk solid. Figure 1.18a provides a survey XP spectrum of the turbostratic graphitic carbon residues obtained upon pyrolysis of polysaccharides where the 
presence of $\mathrm{O}$ can be observed. The relative intensity of the peaks corrected by the instrumental response of the equipment can provide the $\mathrm{C} / \mathrm{O}$ atomic ratio.
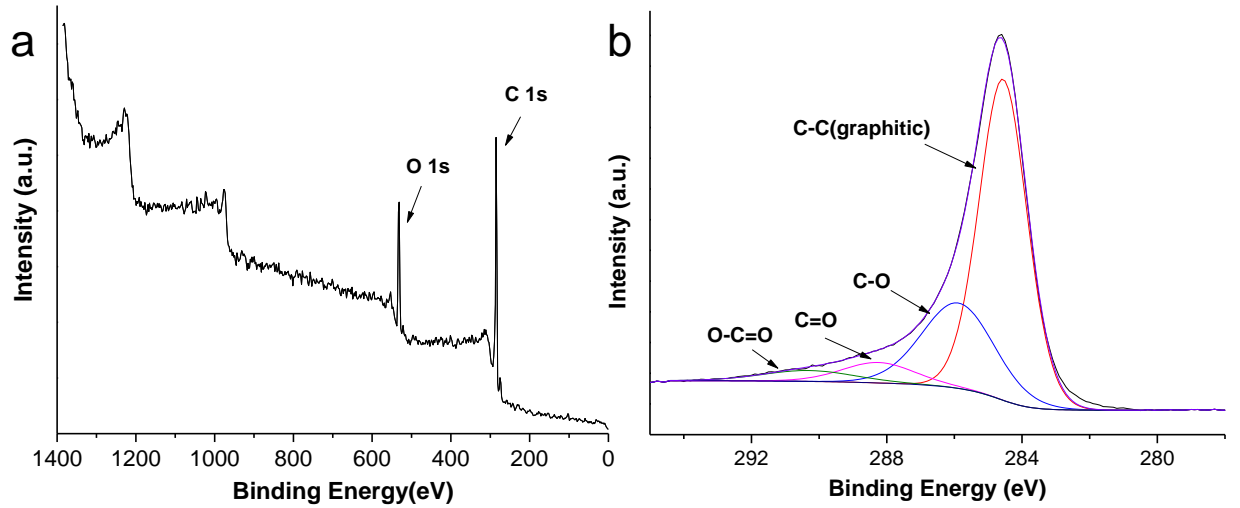

Figure 1.18. The survey XP spectrum (a) and C 1 s peak spectrum (b) of graphitic carbon residues prepared by pyrolysis of alginate.

Besides the survey XP spectrum, the technique allows also the measurement of the peaks corresponding to the different electronic transitions of the various elements with higher resolution. Figure $1.18 \mathrm{~b}$ shows the peak corresponding to $\mathrm{C} 1 \mathrm{~s}$. The shape and width of the experimental XPS peak depends on the contribution of the individual components to this peak. By considering the binding energy of the individual components obtained from defined molecular compounds and by assuming a width of $0.5 \mathrm{eV}$, it is possible to fit the experimental peak as a sum of the individual components in the appropriate proportion. The fitting program estimates these proportions after subtracting the background using an appropriate algorithm. Figure $1.18 \mathrm{~b}$ also presents the $\mathrm{C} 1 \mathrm{~s}$ peak that has been fitted according to the individual components indicated in the plot. As it can be seen there, the major component about $80 \%$ corresponds to graphitic $\mathrm{C}$ atoms appearing at a binding energy of $284.5 \mathrm{eV}$. But the presence of other components corresponding to $\mathrm{C}$ atoms bonded to oxygen with single or double bond with characteristic binding energy at about 285.9 or $288.3 \mathrm{eV}$ respectively and C atoms corresponding to carboxylic acid groups at about $290.2 \mathrm{eV}$ can also be observed in decreasing proportions. Therefore, XPS allows estimating the distribution of the various elements present in the 
defective graphene sample among various coordination environments characterized by different energies.

The information of XPS about the existence of defects can be complemented with the information provided by Raman spectroscopy. This vibrational spectroscopy is very sensitive to graphene and can detect even a single layer of this material. Typically defective graphenes exhibit in Raman spectroscopy three characteristic peaks denoted as $2 \mathrm{D}, \mathrm{G}$ and $\mathrm{D}$ appearing at about 2700, 1590 and $1350 \mathrm{~cm}^{-1}$, respectively, that correspond to the harmonic (2D), the characteristic graphitic peak (G) and defects (D). Figure 1.19 presents a characteristic Raman spectrum corresponding to defective graphene where the presence and shape of these three characteristic peaks can be clearly observed.

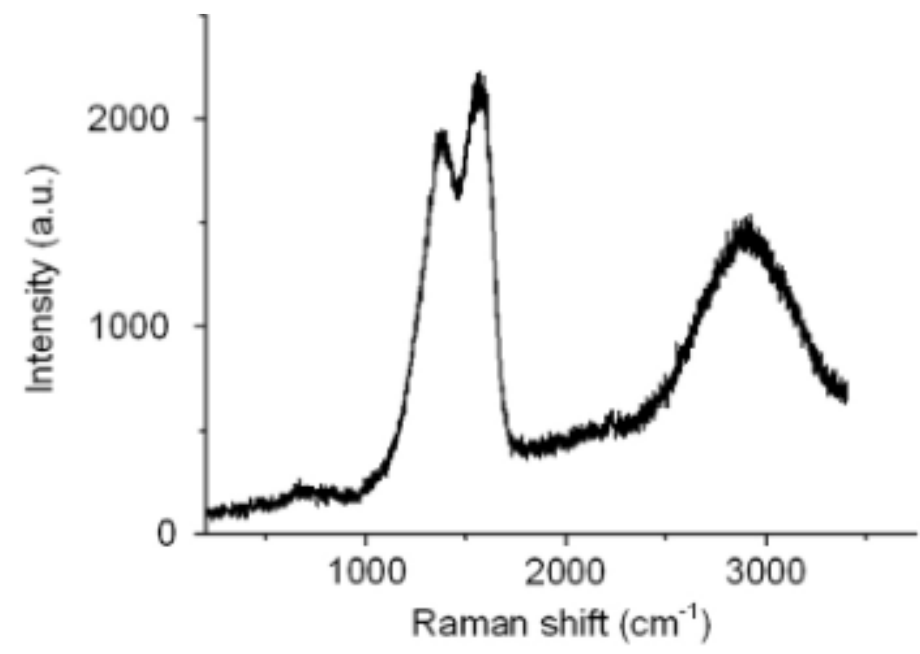

Figure 1.19. Raman spectrum of graphene prepared from chitosan. ${ }^{\text {72] }}$

One simple way to quantify the density of defects on the graphene sheet is by determining the relative intensity of the $G$ vs. the $D$ peak $\left(I_{G} / I_{D}\right)$. Thus, for instance, the value typically reported by this quantitative $I_{G} / I_{D}$ indicator for reduced graphene oxide is about 0.85 , while most of the defective graphenes prepared by pyrolysis of polysaccharides have $I_{G} / I_{D}$ ratios between 1.1 and 1.2. Accordingly, the density of defects of the materials obtained by pyrolysis is smaller than typical value for 
reduced graphene oxides. This parameter will be of importance in Chapter 3 when determining the influence that the presence of $\mathrm{H}_{2}$ and the pyrolysis temperature have over the quality of the obtained defective graphenes.

The pyrolysis method has been adapted to the preparation of doped and co-doped defective graphene samples with heteroatoms that are not present in the precursor. Thus, while chitosan is the precursor of $\mathrm{N}$ in defective graphene and carrageen contains sulfate groups that can become integrated into the defective graphene sample, other heteroatoms like $\mathrm{B}, \mathrm{P}, \mathrm{Si}$, etc can be introduced also in the resulting defective graphene sample by performing the esterification of the natural polysaccharides with an inorganic acid that contains the required element. ${ }^{[78-87]}$ Scheme 1.3 illustrates this strategy. The rich chemistry of carbohydrates allows them to be derivatized and one simple characteristic reaction of hydroxyl groups is esterification with inorganic acids. Among them, boric, phosphoric and silicic acids have been reported to provide defective doped and co-doped graphenes by pyrolysis of the modified polysaccharide. If chitosan already containing $\mathrm{N}$ in its composition is esterified with phosphoric acid, then, the resulting defective graphene contains simultaneously $\mathrm{N}$ and $\mathrm{P}$.

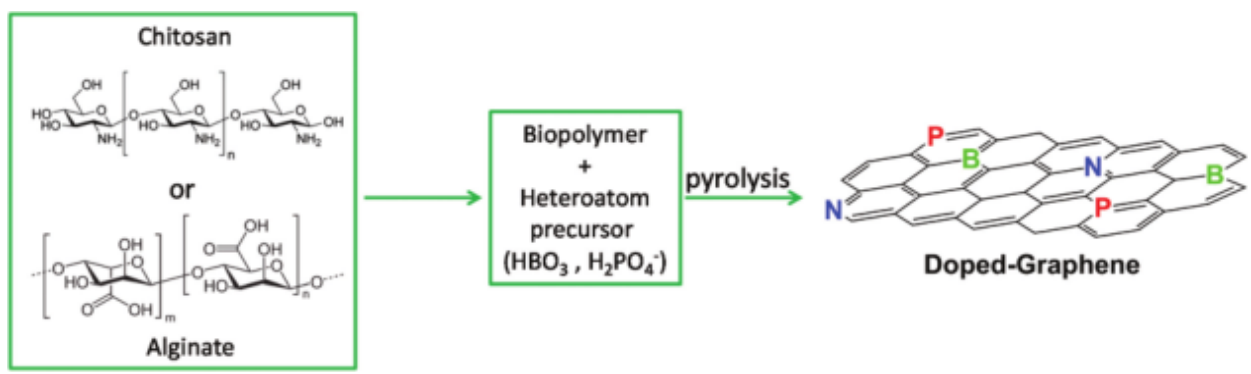

Scheme 1.3. Preparation procedure for doped graphene by pyrolysis of natural polysaccharides (chitosan or alginate) with or without the esterification with inorganic acids. ${ }^{[83]}$

The content of the doped elements has a maximum, but the actual percentage in the final defective graphene can be decreased by increasing the reaction 
temperature. In general, the density of defects decreases with the temperature. In this regard, as previously indicated doping can be considered as a type of defect. It is a target of Chapter 3 to determine quantitatively how the temperature influences the percentage of doping.

\subsection{Heterojunctions of defective graphenes with 2D materials or metal nanoplatelets}

The strategy of pyrolysis of natural polysaccharides has also been adapted to the preparation of other 2D materials as well as some heterojunctions. Other one layer thick materials related to graphenes include boron nitride, transition metal dichalcogenides, phosphorene, silicone, etc. Figure 1.20 illustrates the structures of some of these $2 \mathrm{D}$ materials.

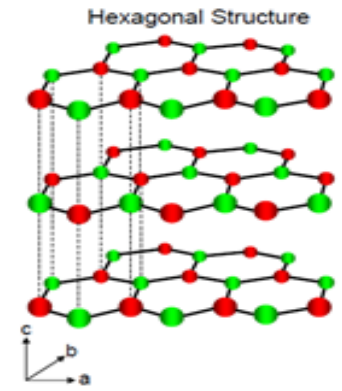

Boron nitride

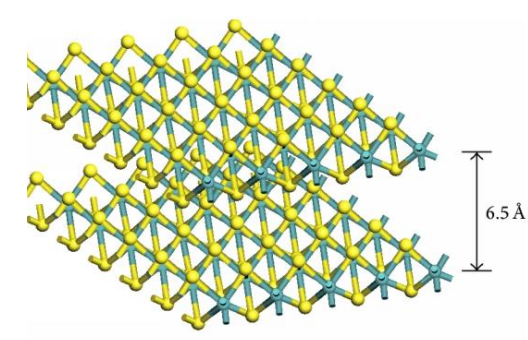

Molybdenum disulfide

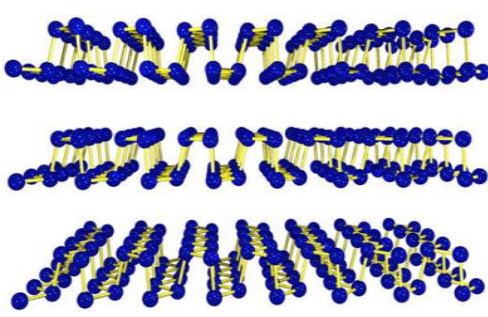

Phosphorene

Figure 1.20. The structures of boron nitride, molybdenum disulfide and phosphrene.

The case of heterojunctions refers to the situation in which two different phases, like defective graphene and another different 2D material, are in intimate contact sharing a considerable fraction of common interface. ${ }^{[88-91]}$ Then, there could be a transfer of electronic density from one material to the other that results in properties for the heterojunction different from those of the individual components. 
Our group reported that when the polysaccharide contains a precursor of boron nitride or molybdenum dichalcogenide, the pyrolysis of these material results in the spontaneous segregation of two independent phases consisting in the defective graphene, as indicated in the previous section, and a second phase. Scheme 1.4 illustrates this strategy forming heterojunctions of graphene-boron nitride and graphene-molybdenum disulfide that have been reported in the literature by our group.

(a)

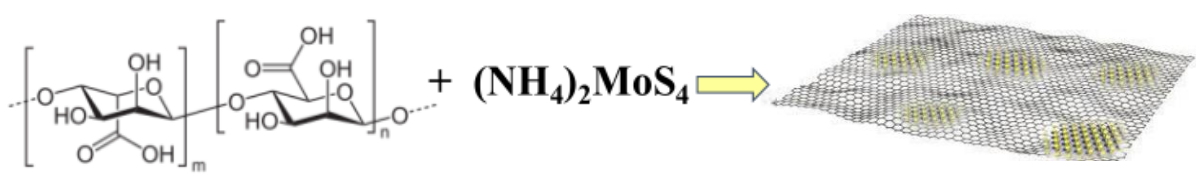

Alginic acid Graphene-MoS

(b)

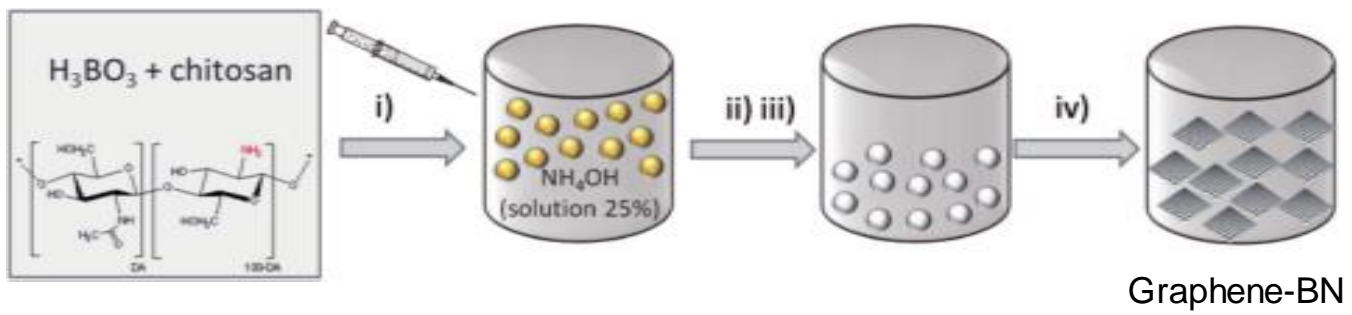

Scheme 1.4. Procedure for the preparation of molybdenum disulfide domains $\left(\mathrm{MoS}_{2}\right)$ on graphene $(\mathbf{a})^{[92]}$ boron nitride on sheets of graphene $(\mathbf{b}) .^{[93]}$

However, the present procedures were used either for powders (case of the heterojunction of $\mathrm{MoS}_{2}$ on graphene) or to obtain the 2D material different from graphene (case of boron nitride). One step forward in this area would be to show that it is possible to prepare films of heterojunctions by applying the method based on the pyrolysis of modified polysaccharides. This is the purpose of Chapters 5 and 6 in where the formation of films of defective graphene/ boron-nitride heterojunctions (Chapter 4) and defective graphene/molybdenum disulfide heterojunctions (Chapter 5) will be described together with the study of their physical and electrochemical properties. 
Another different type of junction refers to metal nanoparticles deposited on graphene. As it is well known, when the sizes of the metal particles decreased to values of a few nanometers, the catalytic activity of these metal NPs will be improved significantly, due to the high percentage of surface atoms with respect to the total number of atoms. These surface atoms contained unsaturated coordination positions in vertices and edges preferably on the surface which make them available to interact with substrates and reagents, promoting the reactions. ${ }^{[94-96]}$

Also, as a result of the presence of these unsaturated atoms, these metal NPs have a high surface energy that makes these particles to tend to suffer agglomeration, which will result in the increase of their size and decrease of the percentage of atoms located on the outer surface. For this reason, most metal particles have sizes in the range of micrometers instead of nanometers. And in this situation the metals in most cases show a low catalytic activity. A paradigmatic example to show this obvious influence of the size of NPs on the catalytic activity is the case of gold metal. The Au NPs with a size of several nanometers are a highly efficient and selective catalyst for some reactions, however, the activity decreases drastically when the particle size turns to higher than $10 \mathrm{~nm} .^{[97-99]}$

In order to stabilize the nanometric dimensions of metal NPs, a common strategy is to deposit the metal NPs on an insoluble support in the reaction medium. This suitable support is supposed to have the characteristics with a high specific surface area and also a strong interaction with the metal NPs to immobilize the NPs and avoid the bleeding of the metal atoms to the reaction medium, as well as the agglomeration of them.

In view of these two considerations, high surface area and strong interaction, it is reasonable to consider the use of graphene as an evolution of the use of active carbons as supports. As indicated before, the graphene has a lager specific surface area which is currently only surpassed by some MOFs. It also has commented that the existence of a $\pi$ cloud extended on the graphene sheet is suitable for interacting with $d$ orbitals of the transition metals. Moreover, the graphene materials may 
provide the additional catalytic sites which have been proposed in a number of researches by showing that graphene in the absence of any matal exhibited certain catalytic activity during oxidation, reduction, acid and base reactions, etc. ${ }^{[26,100-103]}$ Thus, it has been demonstrated both theoretically and experimentally that graphene is a suitable support for metal NPs, including NPs of Pt, Pd, $\mathrm{Au}, \mathrm{Ru}, \mathrm{Ni}, \mathrm{Cu}$, as well as their oxides. ${ }^{[104-107]}$ Figure 1.21 depicts the possible effects for metal NPs from the use of graphene as support.

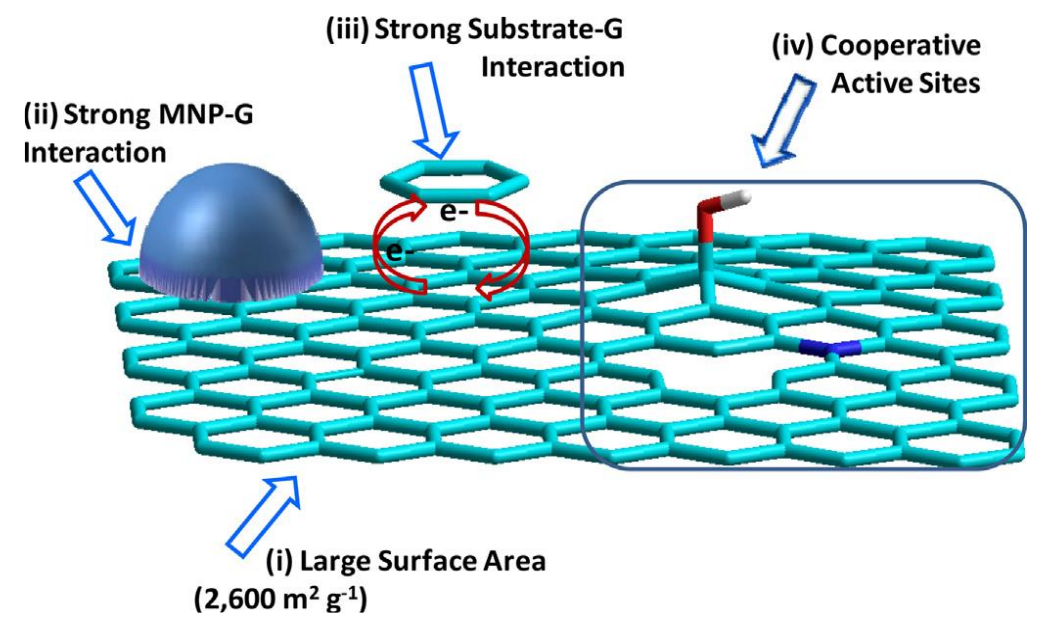

Figure 1.21. Representation of the main effects from graphene when it is used as support for metal NPs in catalysis. ${ }^{[13]}$

The metal NPs supported on $G$ have proven to be better catalysts than other alternatives, using other supports, in oxidation, reduction, coupling, hydrogen release/storage, miscellaneous reactions, and so on. ${ }^{[13]}$ Figure 1.22 shows the results comparing the catalytic activities in various organic reactions from metal NPs supported on graphene with those from metal NPs supported on other types of materials including active carbons, graphite, carbon nanotubes, high surface area metal oxides, or without any support.

Our group has also used the pyrolysis of polysaccharides containing metal salts as a procedure to prepare in one step metal nanoparticles strongly grafted on graphene in those cases in where the formation of metal carbides is not possible. 
Metal carbides are not easily formed in general for late transition metals. This is the case of noble metal (Pt and $\mathrm{Au}$ ) and seminoble metals ( $\mathrm{Ag}$ and $\mathrm{Cu}$ ) that also do not dissolve carbon. In the pyrolytic process, not only carbides are not formed, but also carbon becomes segregated from the metal nanoparticles. ${ }^{[94-100]}$ Scheme 1.5 illustrates the metal nanoparticles-defective graphene materials that have been reported by our group.

These materials containing metals interacting strongly with graphene have very interesting physical and catalytic properties. For instance, it was observed that the morphology of the particles is more like a thin platelet of a few $\mathrm{nm}$ height with $20-40$ $\mathrm{nm}$ lateral dimension and that they develop preferentially a 111 facet. ${ }^{[114-119]}$ This shape of nanoplatelet has been interpreted as indicative of the favorable interaction between the metal and the graphene maximizing the contact area. The preferential growth of the 111 facet that matches the graphene sheet could indicate an epitaxial growth of the metal on the graphene sheet, reflecting again a positive interaction. The consequence of this strong metal-graphene interaction is a (photo)catalytic activity that is frequently several orders of magnitude higher than analogous materials prepared by deposition of preformed metal nanoparticles on preformed graphene. Scheme 1.6 shows the preparation process for oriented metal nanplatelets supported on defective graphenes based on the pyrolytic formation from polysaccharides while as an example, Scheme 1.7 demonstrates the proposed mechanism of preferential growth of $\mathrm{Cu}$ nanoparticles on graphene. 
(a) Aerobic oxidation of methanol to methyl formate

\begin{tabular}{|c|c|c|c|c|c|}
\hline \multirow{5}{*}{$\mathrm{CH}_{3} \mathrm{OH}$} & \multirow{4}{*}{$\stackrel{\text { Cat. }}{\longrightarrow} \mathrm{HCOOCH}_{3}$} & Catalyst & Conversion (\%) & Selectivity (\%) & $\operatorname{TOF}\left(\mathrm{s}^{-1}\right)$ \\
\hline & & $\mathrm{Au}_{2}-\mathrm{Pd}_{1} / \mathrm{rGO}$ & 90.2 & 100 & 0.377 \\
\hline & & $\mathrm{Au}_{2}-\mathrm{Pd}_{1} / \mathrm{Al}_{2} \mathrm{O}_{3}$ & 74.9 & 41.2 & 0.011 \\
\hline & & $\mathrm{Au}_{2}-\mathrm{Pd}_{1} / \mathrm{CNTS}$ & 17.1 & 100 & 0.072 \\
\hline & & $\mathrm{Au}_{2}-\mathrm{Pd}_{1} / \mathrm{TiO}_{2}$ & 33.8 & 19.7 & 0.002 \\
\hline
\end{tabular}

(b) Hydrogenation of isophorone to dihydroisophorone

\begin{tabular}{|c|c|c|c|c|}
\hline & Catalyst & Conversion (\%) & Selectivity (\%) & $\mathrm{TOF}\left(\mathrm{s}^{-1}\right)$ \\
\hline
\end{tabular}

(c) Dehydrogenative coupling reaction

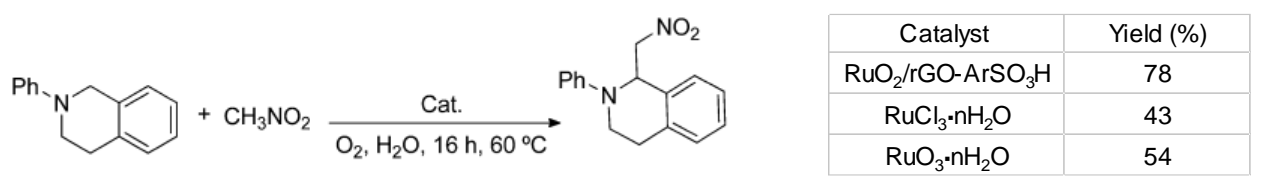

(d) Suzuki-Miyaura cross-coupling reaction

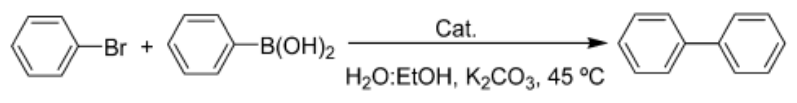

\begin{tabular}{|c|c|c|}
\hline Catalyst & Yield $(\%)$ & TOF $\left(\mathrm{h}^{-1}\right)$ \\
\hline $\mathrm{Pd}-\mathrm{Ni}_{2} \mathrm{O}_{3} / \mathrm{rGO}$ & 99 & 38,750 \\
\hline $\mathrm{Pd} / \mathrm{rGO}$ & 54 & \\
\hline $\mathrm{Ni}_{2} \mathrm{O}_{3} / \mathrm{rGO}$ & 0 & \\
\hline $\mathrm{PdCl}_{2}$ & 57 & \\
\hline $\mathrm{PdCl}_{2}\left(\mathrm{PPh}_{3}\right)_{2}$ & 92 & \\
\hline
\end{tabular}

(e ) Three-component coupling of aldehyde, a secondary amine, and a terminal alkyne

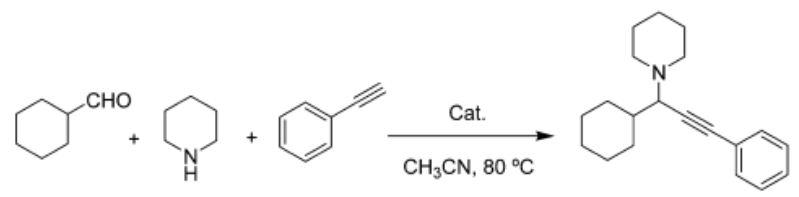

\begin{tabular}{|c|c|}
\hline Catalyst & Yield (\%) \\
\hline $\mathrm{Fe}_{3} \mathrm{O}_{4} / \mathrm{rGO}$ & 90 \\
\hline $\mathrm{Fe}_{3} \mathrm{O}_{4}$ & 45 \\
\hline $\mathrm{Y}-\mathrm{Fe}_{2} \mathrm{O}_{3}$ & 48 \\
\hline
\end{tabular}

(f) Miscellaneous coupling reaction

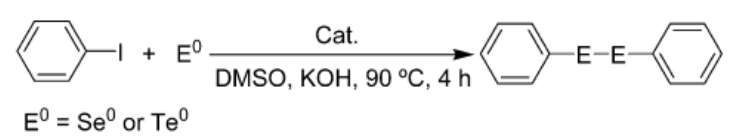

\begin{tabular}{|c|c|c|}
\hline Catalyst & Time $(\mathrm{h})$ & Yield $(\%)$ \\
\hline $\mathrm{Fe}_{3} \mathrm{O}_{4} / \mathrm{rGO}$ & 4 & $96-98$ \\
\hline $\mathrm{Fe}_{3} \mathrm{O}_{4} \mathrm{NPS}$ & 24 & $60-63$ \\
\hline
\end{tabular}

Figure 1.22. Different types of reactions catalyzed by metal NPs supported on graphene which present greater catalytic activity with respect to the same metal NPs on other supports or without support. (a) Aerobic oxidation of methanol to methyl formate, ${ }^{[108]}$ (b) hydrogenation of isophorone to dihydroisophorone, ${ }^{[109]}$ (c) dehydrogenative coupling reaction, ${ }^{[110]}$ (d) suzuki-Miyaura cross-coupling reaction, ${ }^{[111]}$ (e) three-component coupling ${ }^{[112]}$ and (f) miscellaneous coupling reaction. ${ }^{[113]}$ 


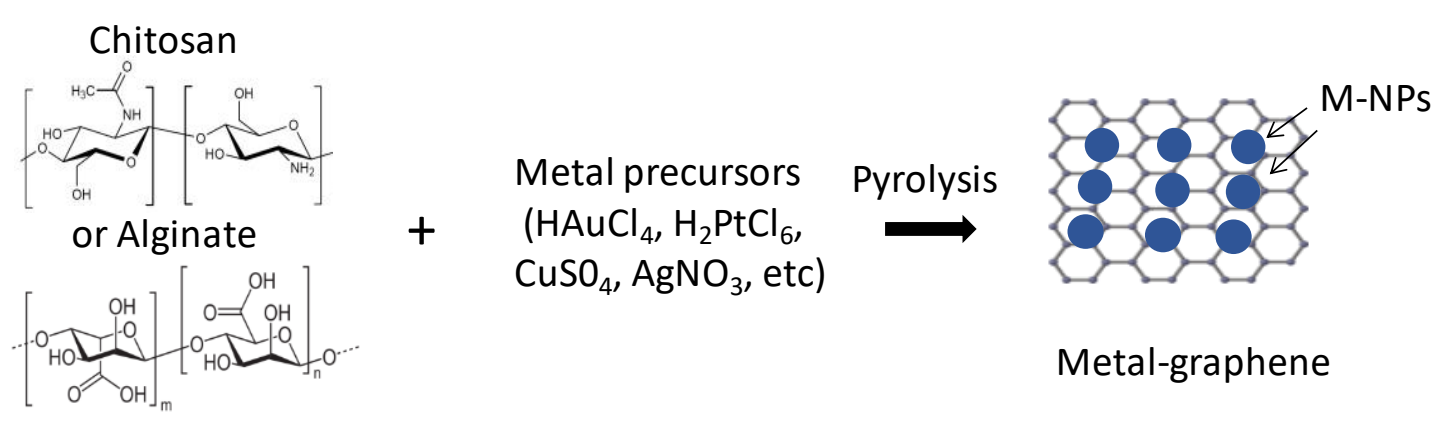

Scheme 1.5. Preparation procedure for various metal nanoparticles supported on defective graphene by pyrolysis of polysaccharides containing metal salts.

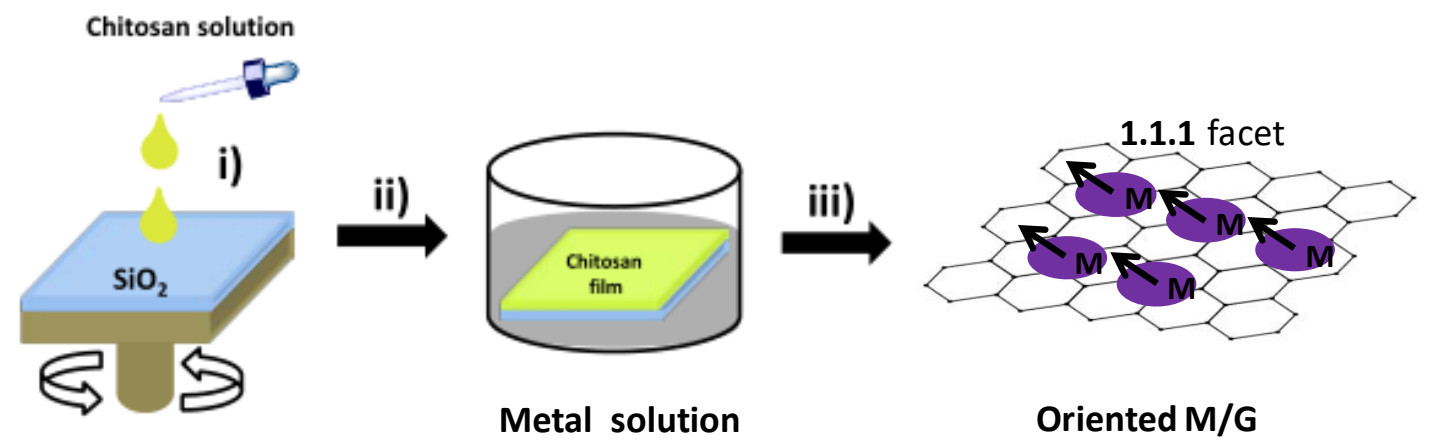

Scheme 1.6. Film preparation process of oriented metals ( $\mathrm{Cu}, \mathrm{Au}, \mathrm{Pt}, \mathrm{Ag}$, etc) supported on G. (i) spin coating on a quartz crystal (dimensions, $2 \times 2 \mathrm{~cm}^{2}$ ) with an aqueous solution of chitosan; (ii) adsorption of metal solution on the film of chitosan, followed by (iii) pyrolysis under an inert atmosphere at $900^{\circ} \mathrm{C}$. 


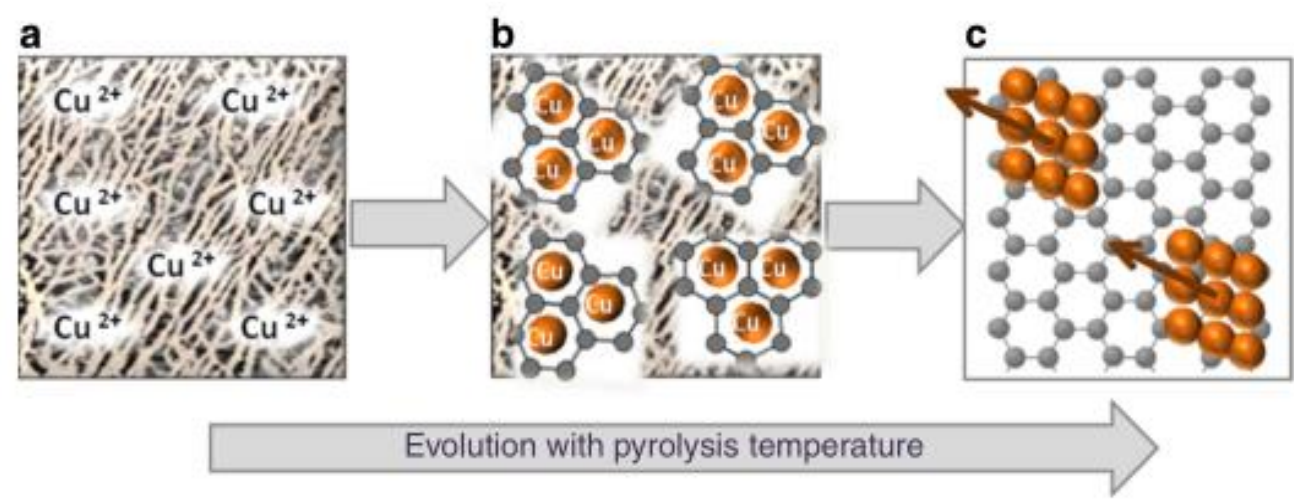

Scheme 1.7. Graphic illustration of the mechanism of oriented $\mathrm{Cu} / \mathrm{G}$ formation. (a) Individual Cu bivalent ions interacting with the fibrils of chitosan. (b) Cu bivalent ions being reduced to $\mathrm{Cu}(0)$ that is accommodated within the hexagonal arrangement of synchronously formed graphene sheet. (c) The preferential growth of $\mathrm{Cu}$ particles along the 1.1.1 facet templated by $\mathrm{G}$ sheet. ${ }^{[117]}$

A logical evolution in this research line would be to carry out and study the properties of these metal-graphene materials for other transition metals including those that can form carbides under high temperature conditions. A study for the case of iron and cobalt has been carried out in the present Doctoral Thesis and is described in Chapters 6 and 7. In the first case, the Fe nanoplatelets on carbon matrix have been used as catalysts for the oxidative C-N coupling of amides (Chapter 6), while in the second case, the formation of Fe and Co nanoplatelets and their alloys have been prepared in a single step by pyrolysis and the resulting materials have been used as catalysts for $\mathrm{CO}_{2}$ hydrogenation (Chapter 7). It appears that higher temperatures or different conditions are required for the formation of these unwanted carbides. As consequence, the iron, cobalt or iron-cobalt nanoparticles embedded within a carbonaceous matrix exhibit remarkable catalytic activity in oxidation and reduction reactions. 


\subsection{References}

[1] Novoselov, K. S.; Geim, A. K.; Morozov, S. V.; Jiang, D.; Zhang, Y.; Dubonos, S. V.; Grigorieva, I. V.; Firsov, A. A. Electric field effect in atomically thin carbon films. Science 2004, 306(5696), 666-669.

[2] Novoselov, K. S.; Geim, A. K.; Morozov, S. V.; Jiang, D.; Katsnelson, M. I.; Grigorieva, I. V.; Dubonos, S. V.; Firsov, A. A. Two-dimensional gas of massless Dirac fermions in graphene. Nature 2005, 438(7065), 197.

[3] Novoselov, K. S.; Jiang, D.; Schedin, F.; Booth, T. J.; Khotkevich, V. V.; Morozov, S. V.; Geim, A. K. Two-dimensional atomic crystals. Proceedings of the National Academy of Sciences of the United States of America 2005, 102(30), 10451-10453.

[4] Bragg, W. H.; Bragg, W. L. The reflection of X-rays by crystals. Proceedings of the Royal Society of London. Series A 1913, 88(605), 428-438.

[5] Girgis, B. S; Temerk, Y. M.; Gadelrab, M. M.; Abdullah, I. D. X-ray diffraction patterns of activated carbons prepared under various conditions. Carbon Letters 2007, 8(2), 95-100.

[6] Li, Z. Q.; Lu, C. J.; Xia, Z. P.; Zhou, Y.; Luo, Z. X-ray diffraction patterns of graphite and turbostratic carbon. Carbon 2007, 45(8), 1686-1695.

[7] Peigney, A.; Laurent, C.; Flahaut, E.; Bacsa, R. R.; Rousset, A. Specific surface area of carbon nanotubes and bundles of carbon nanotubes. Carbon 2001, 39(4), 507-514.

[8] Park, S.; Ruoff, R. S. Chemical methods for the production of graphenes. Nature Nanotechnology 2009, 4(4), 217.

[9] Georgakilas, V.; Otyepka, M.; Bourlinos, A. B.; Chandra, V.; Kim, N.; Kemp, K. C.; Hobza, P.; Zboril, R.; Kim, K. S. Functionalization of graphene: covalent and non-covalent approaches, derivatives and applications. Chemical Reviews 2012, 112(11), 6156-6214.

[10] Cao, M.; Fu, A.; Wang, Z.; Liu, J.; Kong, N.; Zong, X.; Liu, H.; Gooding, J. J. Electrochemical and theoretical study of $\pi-\pi$ stacking interactions between graphitic surfaces and pyrene derivatives. The Journal of Physical Chemistry C 2014, 118(5), 
2650-2659.

[11] Mann, J. A.; Rodríguez-López, J.; Abruña, H. D.; Dichtel, W. R. Multivalent binding motifs for the noncovalent functionalization of graphene. Journal of the American Chemical Society 2011, 133(44), 17614-17617.

[12] Xu, Y.; Bai, H.; Lu, G.; Li, C.; Shi, G. Flexible graphene films via the filtration of water-soluble noncovalent functionalized graphene sheets. Journal of the American Chemical Society 2008, 130(18), 5856-5857.

[13] Navalon, S.; Dhakshinamoorthy, A.; Alvaro, M.; Garcia, H. Metal nanoparticles supported on two-dimensional graphenes as heterogeneous catalysts. Coordination Chemistry Reviews 2016, 312, 99-148.

[14] Sabater, S.; Mata, J. A.; Peris, E. Immobilization of pyrene-tagged palladium and ruthenium complexes onto reduced graphene oxide: an efficient and highly recyclable catalyst for hydrodefluorination. Organometallics 2015, 34(7), 1186-1190. [15] Sabater, S., Mata, J. A.; Peris, E. Catalyst enhancement and recyclability by immobilization of metal complexes onto graphene surface by noncovalent interactions. ACS Catalysis 2014, 4(6), 2038-2047.

[16] Bagri, A.; Mattevi, C.; Acik, M.; Chabal, Y. J.; Chhowalla, M.; Shenoy, V. B. Structural evolution during the reduction of chemically derived graphene oxide. Nature Chemistry 2010, 2(7), 581-587.

[17] Banhart, F.; Kotakoski, J.; Krasheninnikov, A. V. Structural defects in graphene. ACS Nano 2011, 5(1), 26-41.

[18] Jia, Y.; Zhang, L.; Du, A.; Gao, G.; Chen, J.; Yan, X.; Yao, X. Defect graphene as a trifunctional catalyst for electrochemical reactions. Advanced Materials 2016, 28(43), 9532-9538.

[19] Liu, L.; Qing, M.; Wang, Y.; Chen, S. Defects in graphene: generation, healing, and their effects on the properties of graphene: a review. Journal of Materials Science and Technology 2015, 31(6), 599-606.

[20] Araujo, P. T.; Terrones, M.; Dresselhaus, M. S. Defects and impurities in graphene-like materials. Materials Today 2012, 15(3), 98-109.

[21] Agnoli, S.; Favaro, M. Doping graphene with boron: a review of synthesis 
methods, physicochemical characterization, and emerging applications. Journal of Materials Chemistry A 2016, 4(14), 5002-5025.

[22] Kakaei, K.; Balavandi, A. Synthesis of halogen-doped reduced graphene oxide nanosheets as highly efficient metal-free electrocatalyst for oxygen reduction reaction. Journal of Colloid and Interface Science 2016, 463, 46-54.

[23] Tang, Y.; Liu, Z.; Dai, X.; Yang, Z.; Chen, W.; Ma, D.; Lu, Z. Theoretical study on the Si-doped graphene as an efficient metal-free catalyst for CO oxidation. Applied Surface Science 2014, 308, 402-407.

[24] Wang, L.; Sofer, Z.; Luxa, J.; Pumera, M. Nitrogen doped graphene: influence of precursors and conditions of the synthesis. Journal of Materials Chemistry C 2014, 2(16), 2887-2893.

[25] Yang, Z.; Yao, Z.; Li, G.; Fang, G.; Nie, H.; Liu, Z.; Huang, S. Sulfur-doped graphene as an efficient metal-free cathode catalyst for oxygen reduction. ACS Nano 2012, 6(1), 205-211.

[26] Navalon, S.; Dhakshinamoorthy, A.; Alvaro, M.; Garcia, H. Carbocatalysis by graphene-based materials. Chemical Reviews 2014, 114(12), 6179-6212.

[27] Geim, A. K.; Novoselov, K. S. The rise of graphene. Nature Materials 2007, 6(3), 183-191.

[28] Bolotin, K. I.; Sikes, K. J.; Jiang, Z.; Klima, M.; Fudenberg, G.; Hone, J.; Kim, P.; Stormer, H. L. Ultrahigh electron mobility in suspended graphene. Solid State Communications 2008, 146(9), 351-355.

[29] Guo, B.; Fang, L.; Zhang, B.; Gong, J. R. Graphene doping: a review. Insciences Journal 2011, 1(2), 80-89.

[30] Lu, G.; Yu, K.; Wen, Z.; Chen, J. Semiconducting graphene: converting graphene from semimetal to semiconductor. Nanoscale 2013, 5(4), 1353-1368.

[31] Mattson, E. C.; Pu, H.; Cui, S.; Schofield, M. A.; Rhim, S.; Lu, G.; Hirschmugl, C. J. Evidence of nanocrystalline semiconducting graphene monoxide during thermal reduction of graphene oxide in vacuum. ACS Nano 2011, 5(12), 9710-9717.

[32] Prestigiacomo, J. C.; Nath, A.; Osofsky, M. S.; Hernández, S. C.; Wheeler, V. D.; Walton, S. G.; Gaskill, D. K. Determining the nature of the gap in semiconducting 
graphene. Scientific Reports 2017, 7(February), 1-5.

[33] Garg, R.; Dutta, N.; Choudhury, N. Work function engineering of graphene. Nanomaterials 2014, 4(2), 267-300.

[34] Lee, H.; Liu, W.; Chai, S.; Mohamed, A.; Aziz, A.; Khe, C.; Hashim, U. Review of the synthesis, transfer, characterization and growth mechanisms of single and multilayer graphene. RSC Advances 2017, 7(26), 15644-15693.

[35] Wang, H.; Huang, S.; Yang, J.; Wu, G; Hsieh, Y.; Feng, S.; Kuo, C. Large-area few-layered graphene film determination by multispectral imaging microscopy. Nanoscale 2015, 7(19), 9033-9039.

[36] Lin, W.; Chen, T.; Chang, J.; Taur, J.; Lo, Y.; Lee, W.; Chang, C.; Su, W.; Wu, C. A direct and polymer-free method for transferring graphene grown by chemical vapor deposition to any substrate. ACS Nano 2014, 8(2), 1784-1791.

[37] Li, X.; Cai, W.; An, J.; Kim, S.; Nah, J.; Yang, D.; Piner, R.; Velamakanni, A.; Jung, I.; Tutuc. Large-area synthesis of high-quality and uniform graphene films on copper foils. Science 2009, 324(5932), 1312-1314.

[38] Han, G. H.; Gunes, F.; Bae, J. J.; Kim, E. S.; Chae, S. J.; Shin, H. J.; Lee, Y. H. Influence of copper morphology in forming nucleation seeds for graphene growth. Nano Letters 2011, 11(10), 4144-4148.

[39] Reina, A.; Jia, X.; Ho, J.; Nezich, D.; Son, H.; Bulovic, V.; Dresselhaus, M. S.; Kong, J. Large area, few-layer graphene films on arbitrary substrates by chemical vapor deposition. Nano Letters 2008, 9(1), 30-35.

[40] Li, X.; Magnuson, C. W.; Venugopal, A.; An, J.; Suk, J. W.; Han, B.; Ruoff, R. S. Graphene films with large domain size by a two-step chemical vapor deposition process. Nano Letters 2010, 10(11), 4328-4334.

[41] Zhang, Y.; Zhang, L.; Zhou, C. Review of chemical vapor deposition of graphene and related applications. Accounts of Chemical Research 2013, 46(10), 2329-2339.

[42] Kraus, J.; Bobel, L.; Zwaschka, G.; Gunther, S. Understanding the reaction kinetics to optimize graphene growth on $\mathrm{Cu}$ by chemical vapor deposition. Annalen Der Physik 2017, 529(11), 1-16. 
[43] Kumar, A.; Huei, C. Synthesis and biomedical applications of graphene: present and future trends. Advances in Graphene Science 2013.

[44] Chen, L.; Hernandez, Y.; Feng, X.; Mullen, K. From nanographene and graphene nanoribbons to graphene sheets: chemical synthesis. Angewandte ChemieInternational Edition 2012, 51(31), 7640-7654.

[45] Khan, U.; Porwal, H.; O’Neill, A.; Nawaz, K.; May, P.; Coleman, J. N. Solvent-exfoliated graphene at extremely high concentration. Langmuir 2011, 27(15), 9077-9082.

[46] Hernandez, Y.; Nicolosi, V.; Lotya, M.; Blighe, F. M.; Sun, Z.; De, S.; McGovern, I. T.; Holland, B.; Byrne, M.; Gun'Ko, Y. K. High-yield production of graphene by liquid-phase exfoliation of graphite. Nature Nanotechnology 2008, 3(9), 563.

[47] Dhakate, S. R.; Chauhan, N.; Sharma, S.; Tawale, J.; Singh, S.; Sahare, P. D.; Mathur, R. B. An approach to produce single and double layer graphene from re-exfoliation of expanded graphite. Carbon 2011, 49(6), 1946-1954.

[48] Nuvoli, D.; Valentini, L.; Alzari, V.; Scognamillo, S.; Bon, S. B.; Piccinini, M.; Mariani, A. High concentration few-layer graphene sheets obtained by liquid phase exfoliation of graphite in ionic liquid. Journal of Materials Chemistry 2011, 21(10), 3428-3431.

[49] Wang, X.; Fulvio, P. F.; Baker, G. A.; Veith, G. M.; Unocic, R. R.; Mahurin, S. M.; Dai, S. Direct exfoliation of natural graphite into micrometre size few layers graphene sheets using ionic liquids. Chemical Communications 2010, 46(25), 4487-4489.

[50] Stankovich, S.; Dikin, D. A.; Dommett, G. H. B.; Kohlhaas, K. M.; Zimney, E. J.; Stach, E. A.; Piner, R. D.; Nguyen, S. T.; Ruoff, R. S. Graphene-based composite materials. Nature 2006, 442(7100), 282.

[51] Stankovich, S.; Piner, R. D.; Chen, X.; Wu, N.; Nguyen, S. T.; Ruoff, R. S. Stable aqueous dispersions of graphitic nanoplatelets via the reduction of exfoliated graphite oxide in the presence of poly(sodium 4-styrenesulfonate). Journal of Materials Chemistry 2006, 16(2), 155-158.

[52] Zhu, Y.; Murali, S.; Cai, W.; Li, X.; Suk, J. W.; Potts, J. R.; Ruoff, R. S. Graphene and graphene oxide: synthesis, properties, and applications. Advanced Materials 
2010, 22(35), 3906-3924.

[53] Hummers, W. S.; Offeman, R. E. Preparation of graphitic oxide. Journal of the American Chemical Society 1958, 80(6), 1339-1339.

[54] Safari, J.; Gandomi-Ravandi, S.; Ashiri, S. Organosilane sulfonated graphene oxide in the Biginelli and Biginelli-like reactions. New Journal of Chemistry 2016, $40(1), 512-520$.

[55] Gao, W.; Alemany, L. B.; Ci, L.; Ajayan, P. M. New insights into the structure and reduction of graphite oxide. Nature Chemistry 2009, 1(5), 403-408.

[55] Stankovich, S.; Piner, R. D.; Chen, X.; Wu, N.; Nguyen, S. T.; Ruoff, R. S. Stable aqueous dispersions of graphitic nanoplatelets via the reduction of exfoliated graphite oxide in the presence of poly(sodium 4-styrenesulfonate). Journal of Materials Chemistry 2006, 16(2), 155-158.

[56] Robinson, J. T.; Zalalutdinov, M.; Baldwin, J. W.; Snow, E. S.; Wei, Z.; Sheehan, P. Wafer-scale reduced graphene oxide films for nanomechanical devices. Nano Letters 2008, 8(10), 3441-3445.

[57] Gilje, S.; Han, S.; Wang, M.; Wang, K. L.; Kaner, R. B. A chemical route to graphene for device applications. Nano Letters 2007, 7(11), 3394-3398.

[58] Qi, X.; Pu, K.; Zhou, X.; Li, H.; Liu, B.; Boey, F. Conjugated-polyelectrolyte functionalized reduced graphene oxide with excellent solubility and stability in polar solvents. Small 2010, 6(5), 663-669.

[69] Some, S.; Kim, Y.; Yoon, Y.; Yoo, H.; Lee, S.; Park, Y.; Lee, H. High-quality reduced graphene oxide by a dual-function chemical reduction and healing process. Scientific Reports 2013, 3, 1929.

[60] Stankovich, S.; Dikin, D. A.; Piner, R. D.; Kohlhaas, K. A.; Kleinhammes, A.; Jia, Y.; Ruoff, R. S. Synthesis of graphene-based nanosheets via chemical reduction of exfoliated graphite oxide. Carbon 2007, 45(7), 1558-1565.

[61] Pei, S.; Cheng, H. M. The reduction of graphene oxide. Carbon 2012, 50(9), 3210-3228.

[62] Wang, X.; Zhi, L.; Mullen, K. Transparent, conductive graphene electrodes for dye-sensitized solar cells. Nano Letters 2008, 8(1), 323-327. 
[63] McAllister, M. J.; Li, J.; Adamson, D. H.; Schniepp, H. C.; Abdala, A. A.; Liu, J. Single sheet functionalized graphene by oxidation and thermal expansion of graphite. Chemistry of Materials 2007, 19(18), 4396-4404.

[64] Wu, Z.; Ren, W.; Gao, L.; Zhao, J.; Chen, Z.; Liu, B. Synthesis of graphene sheets with high electrical conductivity and good thermal stablility by hydrogen arc discharge exfoliation. ACS Nano 2009, 3(2), 411-417.

[65] Pan, D.; Zhang, J.; Li, Z.; Wu, M. Hydrothermal route for cutting graphene sheets into blue-luminescent graphene quantum dots. Advanced Materials 2010, 22(6), 734-738.

[66] Vlassiouk, I.; Regmi, M.; Fulvio, P.; Dai, S.; Datskos, P.; Eres, G.; Smirnov, S. Role of hydrogen in chemical vapor deposition growth of large single-crystal graphene. ACS Nano 2011, 5(7), 6069-6076.

[67] Ruiz-Hitzky, E.; Darder, M.; Fernandes, F. M.; Zatile, E.; Palomares, F. J.; Aranda, P. Supported graphene from natural resources: easy preparation and applications. Advanced Materials 2011, 23(44), 5250-5255.

[68] Kubo, S.; White, R. J.; Yoshizawa, N.; Antonietti, M.; Titirici, M.-M. Ordered carbohydrate-derived porous carbons. Chemistry of Materials 2011, 23(22), 4882-4885.

[69] Ryoo, R.; Joo, S. H.; Jun, S. Synthesis of highly ordered carbon molecular sieves via template-mediated structural transformation. The Journal of Physical Chemistry $B$ 1999, 103(37), 7743-7746.

[70] Zhao, L.; Baccile, N.; Gross, S.; Zhang, Y.; Wei, W.; Sun, Y.; Antonietti, M.; Titirici, M.-M. Sustainable nitrogen-doped carbonaceous materials from biomass derivatives. Carbon 2010, 48(13), 3778-3787.

[71] Titirici, M.-M.; Antonietti, M. Chemistry and materials options of sustainable carbon materials made by hydrothermal carbonization. Chemical Society Reviews 2010, 39(1), 103-116.

[72] Primo, A.; Sánchez, E.; Delgado, J. M.; Garcia, H. High-yield production of $\mathrm{N}$-doped graphitic platelets by aqueous exfoliation of pyrolyzed chitosan. Carbon 2014, 68, 777-783. 
[73] Primo, A.; Atienzar, P.; Sanchez, E.; Maria Delgado, J.; Garcia, H. From biomass wastes to large-area, high-quality, $\mathrm{N}$-doped graphene: catalyst-free carbonization of chitosan coatings on arbitrary substrates. Chemical Communications 2012, 48(74), 9254-9256.

[74] Primo, A.; Forneli, A.; Corma, A.; Garcia, H. From biomass wastes to highly efficient $\mathrm{CO}_{2}$ adsorbents: graphitisation of chitosan and alginate biopolymers. ChemSusChem 2012, 5(11), 2207-2214.

[75] Primo, A.; Parvulescu, V.; Garcia, H. Graphenes as metal-free catalysts with engineered active sites. The Journal of Physical Chemistry Letters 2017, 8(1), 264-278. [76] Emilio Delgado Sanchez; Pedro Atienzar Corvillo; Ana Maria Primo Arnau; Hermenegildo Garcia Gomez; ABENGOA SOLAR NEW TECH SA: Spain, 2012; Vol. ES 2383356 A1.

[77] Ana Maria Primo Arnau; Emílío Sánchez Cortezón; José María Delgado Sánchez; ABENGOA SOLAR NEW TECH SA: Spain, 2012; Vol. ES2471318 (A1).

[78] Dhakshinamoorthy, A.; Primo, A.; Concepcion, P.; Alvaro, M.; Garcia, H. Doped graphene as a metal-free carbocatalyst for the selective aerobic oxidation of benzylic hydrocarbons, cyclooctane and styrene. Chemistry - A European Journal 2013, 19(23), 7547-7554.

[79] Latorre-Sánchez, M.; Primo, A.; Garcia, H. P-doped graphene obtained by pyrolysis of modified alginate as a photocatalyst for hydrogen gfrom water-methanol mixtures. Angewandte Chemie International Edition 2013, 52(45), 11813-11816.

[80] Primo, A.; Neatu, F.; Florea, M.; Parvulescu, V.; Garcia, H. Graphenes in the absence of metals as carbocatalysts for selective acetylene hydrogenation and alkene hydrogenation. Nature Communications 2014, 5, 5291.

[81] Lavorato, C.; Primo, A.; Molinari, R.; Garcia, H. N-doped graphene derived from biomass as a visible-light photocatalyst for hydrogen generation from water/methanol mixtures. Chemistry - A European Journal 2014, 20(1), 187-194.

[82] Dhakshinamoorthy, A.; Latorre-Sanchez, M.; Asiri, A. M.; Primo, A.; Garcia, H. Sulphur-doped graphene as metal-free carbocatalysts for the solventless aerobic oxidation of styrenes. Catalysis Communications 2015, 65, 10-13. 
[83] Esteve-Adell, I.; Crapart, B.; Primo, A.; Serp, P.; Garcia, H. Aqueous phase reforming of glycerol using doped graphenes as metal-free catalysts. Green Chemistry 2017, 19(13), 3061-3068.

[84] Esteve-Adell, I.; Bakker, N.; Primo, A.; Hensen, E. J. M.; Garcia, H. Graphene as metal-free catalyst for aqueous phase reforming of ethylene glycol. Chemistry Select 2017, 2(22), 6338-6343.

[85] Barrejón, M.; Primo, A.; Gómez-Escalonilla, M. J.; Fierro, J. L. G.; Garcia, H.; Langa, F. Covalent functionalization of $\mathrm{N}$-doped graphene by $\mathrm{N}$-alkylation. Chemical Communications 2015, 51(95), 16916-16919.

[86] Dhakshinamoorthy, A.; He, J.; Franconetti, A.; Asiri, A. M.; Primo, A.; Garcia, H. Defective graphene as a metal-free catalyst for chemoselective olefin hydrogenation by hydrazine. Catalysis Science and Technology 2018, 8(6), 1589-1598.

[87] Rizescu, C.; Podolean, I.; Albero, J.; Parvulescu, V. I.; Coman, S. M.; Bucur, C.; Garcia, H. N-Doped graphene as a metal-free catalyst for glucose oxidation to succinic acid. Green Chemistry 2017, 19(8), 1999-2005.

[88] Deng, D.; Novoselov, K. S.; Fu, Q.; Zheng, N.; Tian, Z.; Bao, X. Catalysis with two-dimensional materials and their heterostructures. Nature Nanotechnology 2016, 11(3), 218-230.

[88] Hu, W.; Yang, J. Two-dimensional van der Waals heterojunctions for functional materials and devices. Journal of Materials Chemistry C 2017, 5(47), 12289-12297.

[89] Koppens, F. H. L.; Mueller, T.; Avouris, P.; Ferrari, A. C.; Vitiello, M. S.; Polini, M. Photodetectors based on graphene, other two-dimensional materials and hybrid systems. Nature Nanotechnology 2014, 9(10), 780-793.

[90] Li, M. Y.; Chen, C. H.; Shi, Y.; Li, L. J. Heterostructures based on two-dimensional layered materials and their potential applications. Materials Today 2016, 19(6), 322-335.

[91] Novoselov, K. S.; Mishchenko, A.; Carvalho, A.; Castro Neto, A. H. 2D materials and van der Waals heterostructures. Science 2016, 353(6298), 9439.

[92] Latorre-Sanchez, M.; Esteve-Adell, I.; Primo, A.; Garcia, H. Innovative preparation of $\mathrm{MoS}_{2}$-graphene heterostructures based on alginate containing 
$\left(\mathrm{NH}_{4}\right)_{2} \mathrm{MoS}_{4}$ and their photocatalytic activity for $\mathrm{H}_{2}$ generation. Carbon 2015, 81, 587-596.

[93] Dhakshinamoorthy, A.; Primo, A.; Esteve-Adell, I.; Alvaro, M.; Garcia, H. Boron nitride nanoplatelets as a solid radical initiator for the aerobic oxidation of thiophenol to diphenyldisulfide. Chemcatchem 2015, 7(5), 776-780.

[94] Blandez, J.; Primo, A.; Asiri, A.; Alvaro, M.; Garcia, H. Copper nanoparticles supported on doped graphenes as catalyst for the dehydrogenative oupling of silanes and alcohols. Angewandte Chemie-International Edition 2014, 53(46), 12581-12586.

[94] Tian, N.; Zhou, Z. Y.; Sun, S. G.; Ding, Y.; Wang, Z. L. Synthesis of tetrahexahedral platinum nanocrystals with high-index facets and high electro oxidation activity. Science $2007,316(5825), 732-735$.

[95] Zhou, Z. Y.; Tian, N.; Li, J. T.; Broadwell, I.; Sun, S. G. Nanomaterials of high surface energy with exceptional properties in catalysis and energy storage. Chemical Society Reviews 2011, 40(7), 4167-4185.

[96] Campbell, C. T.; Parker, S. C.; Starr, D. E. The effect of size-dependent nanoparticle energetics on catalyst sintering. Science 2002, 298(5594), 811-814.

[97] Herzing, A. A.; Kiely, C. J.; Carley, A. F.; Landon, P.; Hutchings, G. J. Identification of active gold nanoclusters on iron oxide supports for CO oxidation. Science 2008, 321(5894), 1331-1335.

[98] Haruta, M. When gold is not noble: catalysis by nanoparticles. The Chemical Record 2003, 3(2), 75-87.

[99] Rashkeev, S. N.; Lupini, A. R.; Overbury, S. H.; Pennycook, S. J.; Pantelides, S. T. Role of the nanoscale in catalytic $\mathrm{CO}$ oxidation by supported $\mathrm{Au}$ and $\mathrm{Pt}$ nanostructures. Physical Review B 2007, 76(3), 035438.

[100] Dreyer, D. R.; Bielawski, C. W. Carbocatalysis: heterogeneous carbons finding utility in synthetic chemistry. Chemical Science 2011, 2(7), 1233-1240.

[101] Dreyer, D. R.; Todd, A. D.; Bielawski, C. W. Harnessing the chemistry of graphene oxide. Chemical Society Reviews 2014, 43(15), 5288-5301.

[102] Lam, E.; Luong, J. H. Carbon materials as catalyst supports and catalysts in the transformation of biomass to fuels and chemicals. ACS catalysis 2014, 4(10), 3393- 
3410.

[103] Haag, D. R.; Kung, H. H. Metal free graphene based catalysts: a review. Topics in Catalysis 2014, 57(6-9), 762-773.

[104] Kamat, P. V. Graphene-based nanoarchitectures. Anchoring semiconductor and metal nanoparticles on a two-dimensional carbon support. The Journal of Physical Chemistry Letters 2009, 1(2), 520-527.

[105] Xu, C.; Wang, X.; Zhu, J. Graphene-metal particle nanocomposites. The Journal of Physical Chemistry C 2008, 112(50), 19841-19845.

[106] Vedala, H.; Sorescu, D. C.; Kotchey, G. P; Star, A. Chemical sensitivity of graphene edges decorated with metal nanoparticles. Nano letters 2011, 11(6), 2342-2347.

[107] Zhou, X.; Huang, X.; Qi, X.; Wu, S.; Xue, C.; Boey, F. Y.; Zhang, H. In situ synthesis of metal nanoparticles on single-layer graphene oxide and reduced graphene oxide surfaces. The Journal of Physical Chemistry C 2009, 113(25), 10842-10846.

[108] Wang, R.; Wu, Z.; Chen, C.; Qin, Z.; Zhu, H.; Wang, G.; Wang, H.; Wu, C.; Dong, W.; Fan, W. Graphene-supported Au-Pd bimetallic nanoparticles with excellent catalytic performance in selective oxidation of methanol to methyl formate. Chemical Communications 2013, 49(74), 8250-8252.

[109] Fan, G. Y.; Huang, W. J. Synthesis of ruthenium/reduced graphene oxide composites and application for the selective hydrogenation of halonitroaromatics. Chinese Chemical Letters 2014, 25(2), 359-363.

[110] Meng, Q. Y.; Liu, Q.; Zhong, J. J.; Zhang, H. H.; Li, Z. J.; Chen, B.; Wu, L. Z. Graphene-supported $\mathrm{RuO}_{2}$ nanoparticles for efficient aerobic cross-dehydrogenative coupling reaction in water. Organic Letters 2012, 14(23), 5992-5995.

[111] Nie, R., Shi, J., Du, W., \& Hou, Z. $\mathrm{Ni}_{2} \mathrm{O}_{3}$-around-Pd hybrid on graphene oxide: An efficient catalyst for ligand-free Suzuki-Miyaura coupling reaction. Applied Catalysis A: General 2014, 473, 1-6.

[112] Huo, X.; Liu, J.; Wang, B.; Zhang, H.; Yang, Z.; She, X.; Xi, P. A one-step method to produce graphene- $\mathrm{Fe}_{3} \mathrm{O}_{4}$ composites and their excellent catalytic activities for three-component coupling of aldehyde, alkyne and amine. Journal of Materials 
Chemistry A 2013, 1(3), 651-656.

[113] Kassaee, M. Z.; Motamedi, E.; Movassagh, B.; Poursadeghi, S. Iron-catalyzed formation of $\mathrm{C}-\mathrm{Se}$ and $\mathrm{C}-\mathrm{Te}$ bonds through cross coupling of aryl halides with $\mathrm{Se}(0)$ and $\mathrm{Te}(0) / \mathrm{Nano}^{-} \mathrm{Fe}_{3} \mathrm{O}_{4} @$ GO. Synthesis 2013, 45(16), 2337-2342.

[114] Primo, A.; Esteve-Adell, I.; Coman, S. N.; Candu, N.; Parvulescu, V. I.; Garcia, H. One-step pyrolysis preparation of 1.1 .1 oriented gold nanoplatelets supported on graphene and six orders of magnitude enhancement of the resulting catalytic activity. Angewandte Chemie-International Edition 2016, 55(2), 607-612.

[115] Dhakshinamoorthy, A.; Esteve Adell, I.; Primo, A.; Garcia, H. Enhanced activity of $\mathrm{Ag}$ nanoplatelets on few layers of graphene film with preferential orientation for dehydrogenative silane-alcohol coupling. ACS Sustainable Chemistry \& Engineering $2017,5(3), 2400-2406$.

[116] Esteve-Adell, I.; Bakker, N.; Primo, A.; Hensen, E.; Garcia, H. Oriented Pt nanoparticles supported on few-layers graphene as highly active catalyst for aqueous-phase reforming of ethylene glycol. ACS Applied Materials \& Interfaces 2016, 8(49), 33690-33696.

[117] Primo, A.; Esteve-Adell, I.; Blandez, J. F.; Dhakshinamoorthy, A.; Alvaro, M.; Candu, N.; Coman, S. M.; Parvulescu, V. I.; Garcia, H. High catalytic activity of oriented 2.0.0 copper(I) oxide grown on graphene film. Nature Communications 2015, 6, 8561. [118] Frindy, S.; El Kadib, A.; Lahcini, M.; Primo, A.; Garcia, H. Isotropic and oriented copper nanoparticles supported on graphene as aniline guanylation catalysts. ACS Catalysis 2016, 6(6), 3863-3869.

[119] Mateo, D.; Esteve-Adell, I.; Albero, J.; Sanchez Royo, J. F.; Primo, A.; Garcia, H. 111 oriented gold nanoplatelets on multilayer graphene as visible light photocatalyst for overall water splitting. Nature Communications 2016, 7, 11819. 
Chapter 2

Objectives 

The general objective of the present Doctoral Thesis is to develop heterojunctions of defective graphenes with other different 2D materials and metal nanoparticles by applying natural polysaccharides as precursors of defective graphenes. Within this general context, the specific objectives of each of the Chapters that report original research in the field are the following:

1. Improvement of the quality of defective graphenes by determining the influence of the presence of hydrogen during the pyrolysis at different temperatures. Given the importance of alginate and chitosan in the formation of defective graphenes by pyrolysis, the influence of the presence of hydrogen at different pyrolysis temperatures will be studied for these two natural biopolymers. The purpose is to determine the improvement in conductivity that can be gained by deeper removal of the oxygenated groups during the pyrolysis. Accomplishment of this objective will represent an additional valorization of the process.

2. Formation of films of defective graphene/boron nitride heterojunction. This type of heterojunction is supposed to exhibit an enhanced conductivity as consequence of the favorable electron mobility on graphene when it is supported on boron nitride. Also the possibility to use this heterojunction to store electrostatic charge like in a microcapacitor will be assessed by using microelectrodes to contact the thin film and measuring the current as a function of the applied voltage.

3. Formation of films of defective graphene/MoS 2 heterojunctions that could be used as electrocatalysts without the need of any further conductive material. $\mathrm{MoS}_{2}$ on graphene has been reported as an electrocatalyst with activity close to that of Pt for hydrogen generation. This electrochemical reaction is considered one of the simplest and more convenient reactions to store renewable electricity surplus production into chemicals and it is important to evaluate the performance of the heterojunction obtained by the pyrolysis method starting from natural biopolymers.

4. Formation of metal nanoparticles of iron and cobalt and their alloys supported on defective graphene in one step by pyrolysis of polysaccharides adsorbing salts of 
these two metal in different proportions and characterization of their activity as oxidation catalysts in the oxidative $\mathrm{C}-\mathrm{N}$ coupling of $\mathrm{N}$-methyl amides and aromatic $\mathrm{N}-\mathrm{H}$ compounds. The influence of the composition of the metal nanoparticles in the catalytic activity will be determined.

5. Determination of the catalytic activity of the iron and cobalt nanoparticles and their alloys in various proportions supported on defective graphene as catalysts for $\mathrm{CO}_{2}$ hydrogenation. In the context of the change in the current scheme of energy sources from fossil fuels to renewable energies, there is much interest in implementing a circular economy in which $\mathrm{CO}_{2}$ becomes the feedstock for the production of fuels. The process can be catalyzed by Fe and Co catalysts and it is of interest to compare the activity of materials based on Fe, Co and their alloys supported on defective graphenes with other types of Fe and Co based catalysts reported in the literature.

In case that the specific objectives of the present Doctoral Thesis are achieved, they will represent a considerable advance in the field, since in general, the results will serve to exemplify the valorization of biomass wastes into graphene materials and heterojunctions with higher performance and the current materials or catalysts will show a certain value in organic synthesis or in future renewable energies. 


\section{Chapter 3}

Quality improvement of defective graphene from

biomass

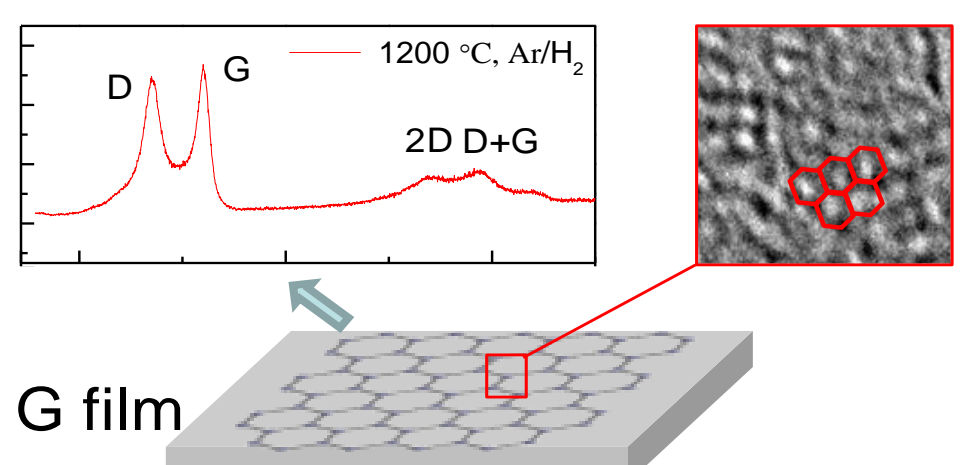





\subsection{Introduction}

There is a considerable interest in the development of different graphene preparation methods that, on one hand, can serve to valorize wastes, while at the same time can render materials with adequate properties for a given application. ${ }^{[1-4]}$ In this context, as it has been commented in the introduction our group reported a few years ago that pyrolysis at temperatures above $900{ }^{\circ} \mathrm{C}$ of alginate and chitosan affords carbon residues that undergo easy exfoliation in high yields to form defective graphenes. ${ }^{[5-7]}$ Starting from certain natural filmogenic polysaccharides as precursors, pyrolysis can serve for the preparation of defective graphenes either as films or as suspensions. ${ }^{[6,7]}$ This procedure can be adapted also to the preparation of other doped graphenes, either by using a precursor that contains already the heteroatom, like chitosan and $\lambda$-carrageen for the preparation of $\mathrm{N}$ - and $\mathrm{S}$-doped defective graphene, ${ }^{[7,8]}$ respectively, or alternatively the polysaccharide can be derivatized by reaction with inorganic acids that contain the required heteroatom. ${ }^{[9]}$ Moreover, as it has been explained earlier the method can also be adapted to the one-step preparation of heterojunctions of differently doped defective graphenes, or heterojunctions of graphene with other $2 \mathrm{D}$ materials and even to the preparation of graphenes having strongly grafted metal nanoparticles (Scheme 3.1). ${ }^{[10-12]}$

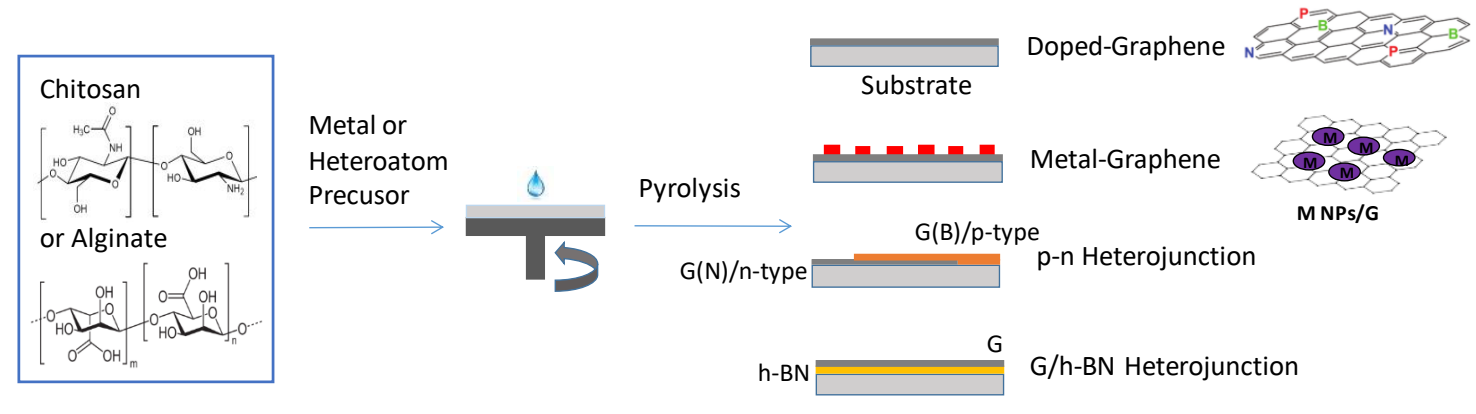

Scheme 3.1. Pictorial illustration of the one-step preparation of doped graphene and various types of graphene heterojunctions.

Some of the defects in this type of graphenes derive from the presence of a 
residual oxygen content, about $20 \%$, remaining from the incomplete carbonization of the polysaccharide precursors that initially contain above $50 \%$ oxygen in their composition. Among the various characterization techniques, it has already been indicated that the presence of defects, including oxygenated functional groups, can be easily monitored by Raman spectroscopy and quantitatively assessed by the shape and relative intensities of the $G$ vs. the $D$ bands appearing at about 1600 and 1350 $\mathrm{cm}^{-1}$, respectively. Typical values of $\mathrm{I}_{\mathrm{G}} / \mathrm{I}_{\mathrm{D}}$ in defective graphenes obtained from natural polysaccharide pyrolysis are about $1.15 .{ }^{[7]}$

Besides Raman spectroscopy, the presence of these defects is typically also reflected in the electrical conductivity of the graphene material. As already commented, high electrical conductivity is relevant for different applications of graphenes related to microelectronics and to the preparation of transparent and conductive displays, among others. ${ }^{[13,14]}$ In the case of defective graphenes obtained from biomass pyrolysis, it has been reported that the electrical conductivity measured by the four-tips head method on quartz substrates is in the range of a few $\mathrm{k} \Omega / \mathrm{sq}$, typically from 3 to $10 \mathrm{k} \Omega / \mathrm{sq}$, significantly lower than the values reported for ideal graphene on other supports. ${ }^{[6]}$

In this context, considering the added value of the conversion of alginate, chitosan and other natural polysaccharides into graphenes, it would be of interest to improve further the electrical conductivity of these defective graphenes obtained by the pyrolysis of biopolymers by introducing some optimization in the preparation procedure. This could open additional applications for this type of graphene materials. Since oxygen functionalities are one type of defect, it was considered that decrease in the oxygen content of these materials should also be reflected in a diminution of the density of defects, at least those caused by oxygenated functional groups.

Aimed at this purpose, it is well-known that the preparation method of graphenes by chemical vapor deposition, as well as some other procedures, are carried out under $\mathrm{H}_{2}$ atmosphere. ${ }^{[15,16]}$ During the high temperature pyrolysis, $\mathrm{H}_{2}$ can 
act as chemical reducing agent and it could produce an additional decrease in the oxygen content of the resulting defective graphene by formation of $\mathrm{H}_{2} \mathrm{O}$ or other hydrogenation volatile compounds and, therefore, its presence during the pyrolytic process could be reflected in an improvement of quality of the films obtained in this way that could be accompanied by an increase in the electrical conductivity. However, the details of the influence of the presence of $\mathrm{H}_{2}$ during the pyrolysis of natural polysaccharides remain unexplored.

In the present Chapter, it will be shown that by carrying out the pyrolysis of alginic acidand chitosan at increasing temperatures in the presence of $\mathrm{H}_{2}$ gas, defective graphenes with lower oxygen content, lower density of defects and improved electrical conductivity and photoelectric catalytic activity can be obtained. The present study represents a step forward in the direction of valorization of biomass wastes by obtaining high added-value graphene films.

\subsection{Results and discussion}

Films of ammonium alginate and chitosan on clean quartz substrates were prepared by spin coating and their pyrolysis was carried out at temperatures in the range from 900 to $1200{ }^{\circ} \mathrm{C}$, either under a flow of $\mathrm{Ar}$ or under $\mathrm{Ar}$ containing 5 or 10 vol\% $\mathrm{H}_{2}$ (see Chapter 8 for the details of experimental procedures) (Scheme 3.2). Preliminary controls revealed that higher pyrolysis temperatures result in an almost complete volatilization of the biopolymer with no graphene material remaining, while higher $\mathrm{H}_{2}$ proportions do not apparently play any additional beneficial role in the quality of the resulting graphene. As commented above, the rationale was to increase the reductive conditions of the carbonization process, leading to a decrease of the residual oxygen content of the defective graphene films formed in the process by evolution of $\mathrm{H}_{2} \mathrm{O}$ or other gases. 


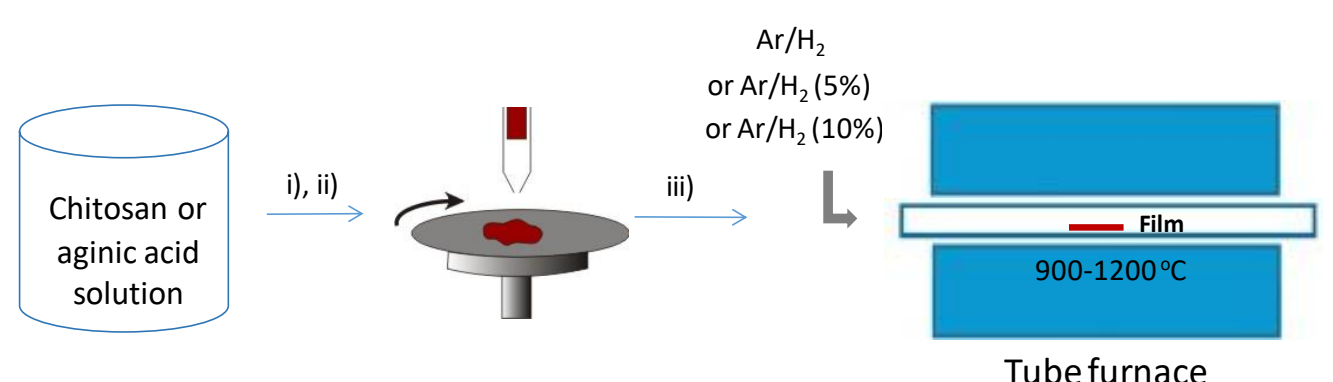

Tube furnace

Scheme 3.2. Steps in the preparation of the graphene or N-doped graphene film on quartz: i) dissolution of chitosan or alginic acid in aqueous solution of acetic acid or ammonia; ii) spin coating and water evaporation; iii) pyrolysis of the sample at different temperatures $\left(900-1200^{\circ} \mathrm{C}\right)$ under $\mathrm{Ar}$, or $\mathrm{Ar} / \mathrm{H}_{2}(5 \%)$, or $\mathrm{Ar} / \mathrm{H}_{2}(10 \%)$ flow.

To assess the influence of the pyrolysis temperature and presence of $\mathrm{H}_{2}$ and its concentration, the resulting films were characterized by Raman spectroscopy, XPS analysis and electrical measurements and tested as electrodes for $\mathrm{H}_{2}$ evolutionreaction. The morphology and thickness of the films were studied by AFM and SEM of the films, as well as TEM of small pieces scratched from the films.

As it was expected in view of the abundant literature data, Raman spectroscopy was a useful and convenient technique to follow the influence of the pyrolysis temperature and $\mathrm{H}_{2}$ concentration on the density of defects present on the resulting graphene film samples. ${ }^{[17-19]}$ Figure 3.1 shows a set of Raman spectra to illustrate the type of changes observed in the characteristic graphene peaks by presenting two extreme cases, while Figures 3.2 and 3.3 include a complete set of Raman spectra for other films prepared in this Chapter.

There are several parameters used to assess the spectral changes occurring under the various preparation conditions, namely, the ratio between the intensity of the $G$ vs. the $D$ bands $\left(I_{G} / I_{D}\right.$ ), the full width at half height of the $G$ and $D$ peaks ( $f w h p_{G}$ and $\left.f w h p_{D}\right)$ and the resolution of the peaks measured by the ratio between the maximum intensity of the $G$ band and the valley between the $G$ and $D$ bands $(R)$. The higher the $I_{G} / I_{D}$ ratio, the narrower the $G$ and $D$ peaks and the higher the resolution 
between the peak and the valley, the higher was assumed to be the quality of the resulting graphene film, according to Raman spectroscopy. The corresponding values of the three parameters determined from the experimental Raman spectra are tabulated in Table 3.1 .
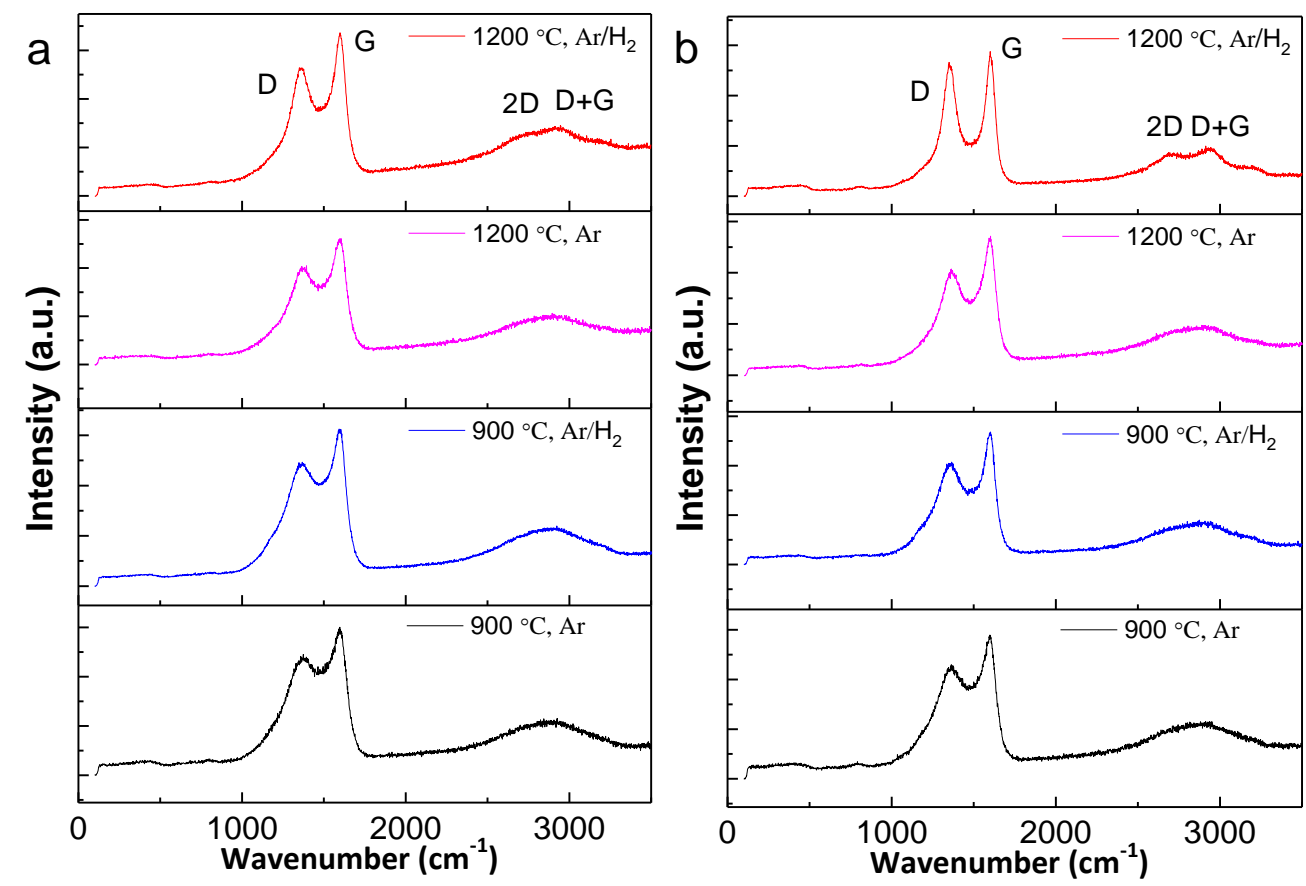

Figure 3.1. Raman spectra of $(N) G$ prepared from chitosan (a) and $G$ prepared from alginic acid(b) pyrolyzed at $900,1200{ }^{\circ} \mathrm{C}$ under $\mathrm{Ar}$ or $\mathrm{Ar} / \mathrm{H}_{2}$ (5\%) flow respectively. 

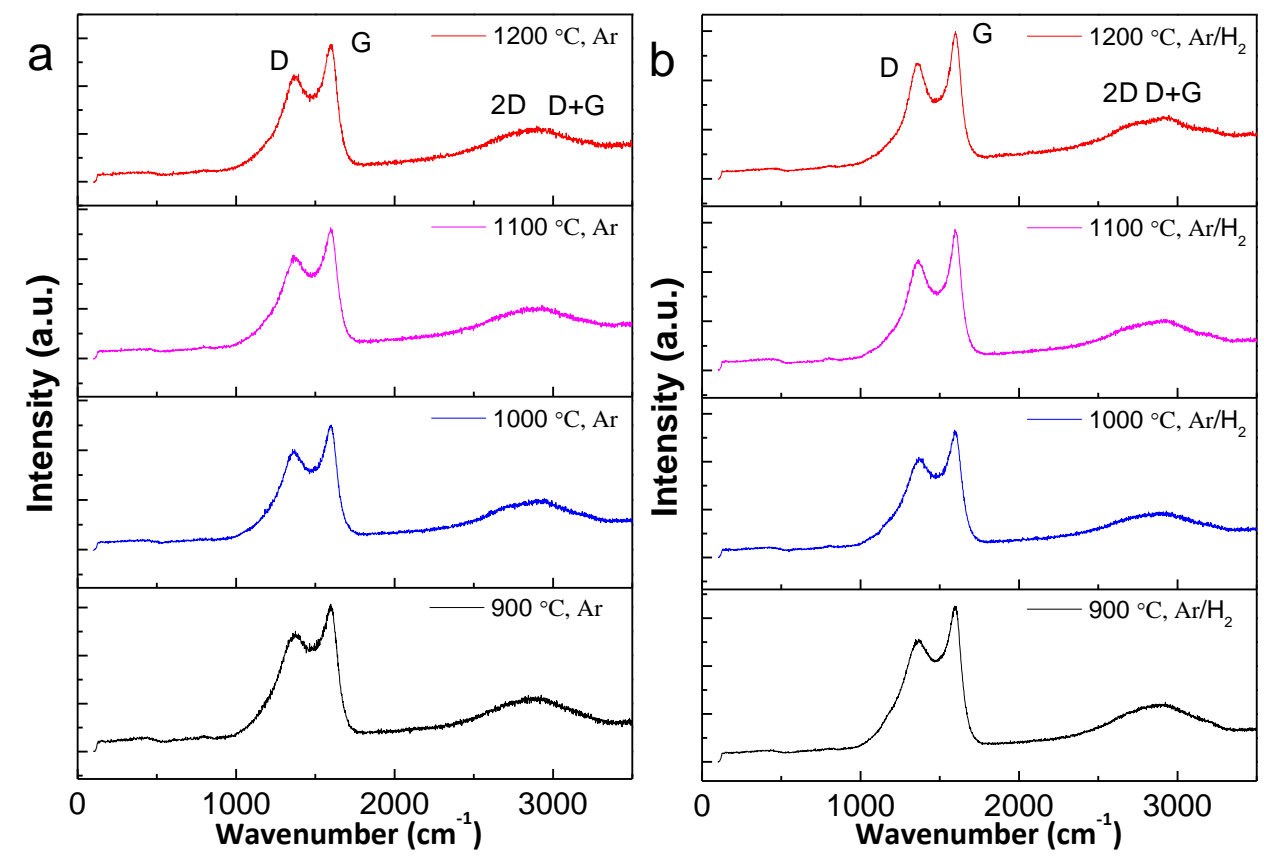

Figure 3.2. Raman spectra of (N)G prepared from chitosan pyrolyzed at 900,1000 , $1100,1200^{\circ} \mathrm{C}$ under $\operatorname{Ar}(\mathrm{a})$ or $\mathrm{Ar} / \mathrm{H}_{2}$ (5\%) (b) flow respectively.
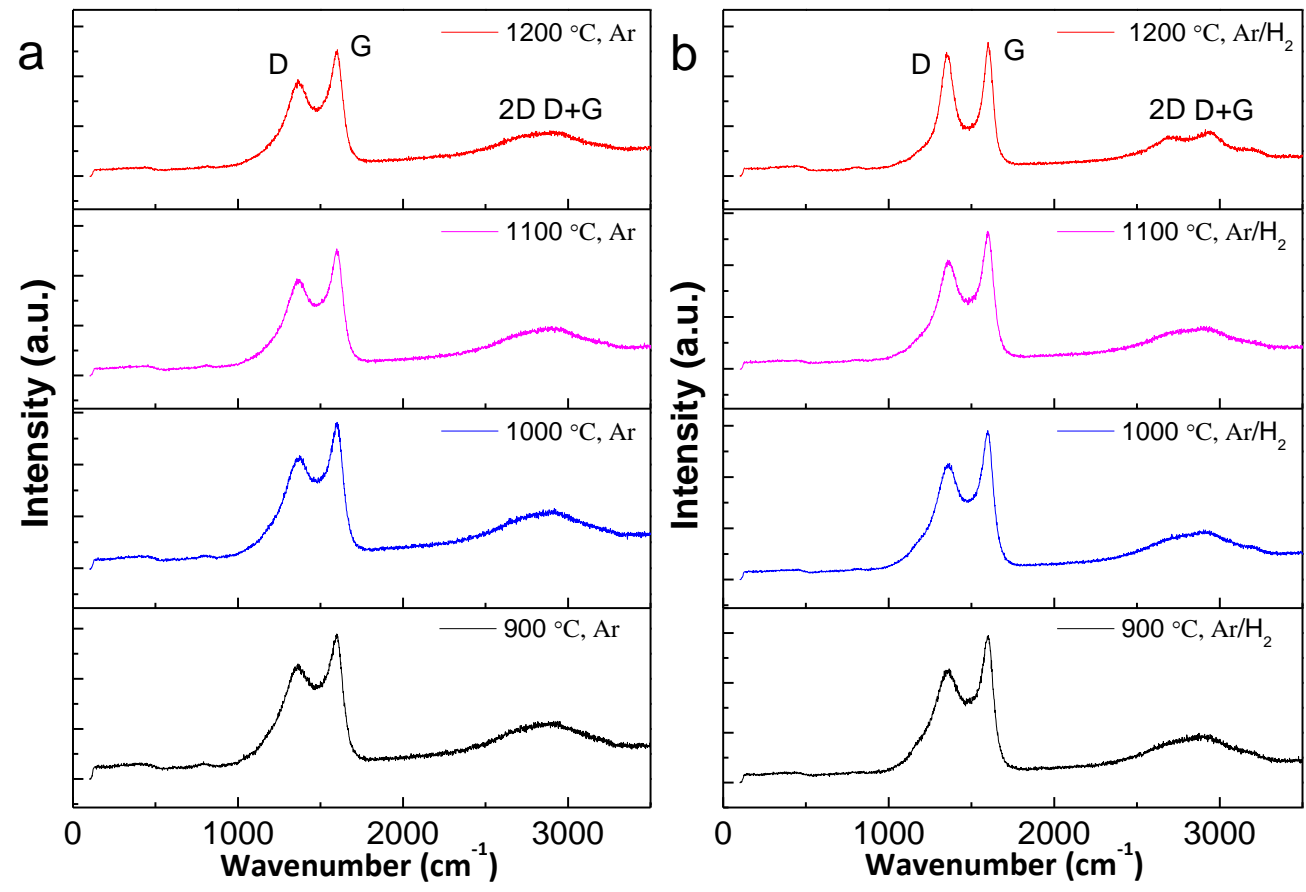

Figure 3.3. Raman spectra of $G$ prepared from alginic acidpyrolyzed at 900,1000 , $1100,1200{ }^{\circ} \mathrm{C}$ under $\mathrm{Ar}(\mathbf{a})$ or $\mathrm{Ar} / \mathrm{H}_{2}(5 \%)$ (b) flow respectively. 
As it can be seen in Table 3.1 and also visually in Figure 3.1, most of the parameters determined by Raman indicate that the highest quality samples, both for alginic acidand chitosan, are achieved when the pyrolysis was carried out at $1200{ }^{\circ} \mathrm{C}$ in the presence of $10 \% \mathrm{H}_{2}$. As shown in Table 3.1, $I_{G} / I_{D}$ ratio is not the most useful parameter to follow these spectroscopic changes (see entries 2 and 3 in Table 3.1) that can be better assessed by fwhp of the $G$ and $D$ peaks and the resolution between peak and valley. It should be noted that a $\mathrm{H}_{2}$ concentration increase from 5 to $10 \%$ during the $12 \mathrm{~h}$ experiment does not substantially improve the Raman quality parameters (see Table 3.1 and Figure 3.4), while it represents twice $\mathrm{H}_{2}$ consumption. On the other hand, temperatures higher than $1200^{\circ} \mathrm{C}$ lead to the complete volatilization of the precursor without any residual graphene film remaining on the quartz plate and, therefore, it appears to be an upper limit on the temperature of the process.

It is particularly worth noting that the spectroscopic changes recorded for alginic acid compared to chitosan, probably reflect that in the last case, $\mathrm{N}$ atoms are still present as dopant element on the resulting graphene $((\mathrm{N}) \mathrm{G})$. The presence of dopant $\mathrm{N}$ heteroatoms should introduce some features (defects) in the Raman spectrum that limit possible decreases in the intensity and/or width of the D band. Accordingly, in the case of graphene derived from alginic acid, much sharper $G$ and $D$ bands were recorded, while the presence of the $2 D$ and $D+G$ overtones appeared also well resolved in the higher frequency region of the spectrum. The results presented in Figures 3.1 to 3.4 and Table 3.1 clearly document the positive influence of optimization of pyrolysis temperature and the presence of $\mathrm{H}_{2}$ on the Raman spectra of the resulting graphene films. These spectral changes are associated to an improvement on the quality of the graphene films as consequence of their lower oxygen content. 
Table 3.1. Parameters determined from the Raman spectra to assess the influence of the pyrolysis temperature and $\mathrm{H}_{2}$ concentration on the defect density on the resulting graphene films. ${ }^{a}$

\begin{tabular}{|c|c|c|c|c|c|c|c|c|c|c|}
\hline & & & \multicolumn{4}{|c|}{$(N) G$} & \multicolumn{4}{|c|}{ G } \\
\hline Entry & $\mathrm{T}\left({ }^{\circ} \mathrm{C}\right)$ & Gas & $\mathrm{I}_{G} / \mathrm{I}_{\mathrm{D}}$ & $\mathrm{fwhp}_{G}$ & $f w h p_{D}$ & $\mathrm{R}$ & $\mathrm{I}_{G} / \mathrm{I}_{\mathrm{D}}$ & $\mathrm{fwhp}_{G}$ & fwhp & $\mathrm{R}$ \\
\hline 1 & 900 & $\mathrm{Ar}$ & 1.26 & 176.2 & 331.0 & 1.57 & 1.26 & 155.3 & 330.1 & 1.70 \\
\hline 2 & 900 & $\mathrm{Ar} / \mathrm{H}_{2}(5 \%)$ & 1.27 & 156.6 & 291.9 & 1.68 & 1.31 & 114.2 & 275.9 & 2.03 \\
\hline 3 & 1000 & $\mathrm{Ar}$ & 1.26 & 161.6 & 304.2 & 1.75 & 1.31 & 141.5 & 301.9 & 1.82 \\
\hline 4 & 1000 & $\mathrm{Ar} / \mathrm{H}_{2}(5 \%)$ & 1.26 & 149.4 & 247.1 & 1.76 & 1.28 & 113.6 & 254.0 & 2.07 \\
\hline 5 & 1100 & $\mathrm{Ar}$ & 1.30 & 144.0 & 307.1 & 1.76 & 1.28 & 135.9 & 257.3 & 1.93 \\
\hline 6 & 1100 & $\mathrm{Ar} / \mathrm{H}_{2}(5 \%)$ & 1.29 & 119.0 & 214.3 & 2.03 & 1.26 & 105.2 & 216.9 & 2.31 \\
\hline 7 & 1100 & $\left.\mathrm{Ar} / \mathrm{H}_{2} 10 \%\right)$ & 1.29 & 115.2 & 208.1 & 2.07 & 1.29 & 98.9 & 208.5 & 2.36 \\
\hline 8 & 1200 & $\mathrm{Ar}$ & 1.31 & 138.2 & 296.2 & 1.79 & 1.30 & 120.2 & 221.2 & 2.07 \\
\hline 9 & 1200 & $\mathrm{Ar} / \mathrm{H}_{2}(5 \%)$ & 1.31 & 116.5 & 214.6 & 2.15 & 1.08 & 89.6 & 122.4 & 3.18 \\
\hline 10 & 1200 & $\left.\mathrm{Ar} / \mathrm{H}_{2} 10 \%\right)$ & 1.31 & 112.6 & 216.8 & 2.21 & 1.13 & 87.4 & 121.4 & 3.22 \\
\hline
\end{tabular}

[a] fwhp: full width at half peak height; R: resolution between the $G$ and the $D$ peak. The gray cells highlight the best values according to each quality indicator. 

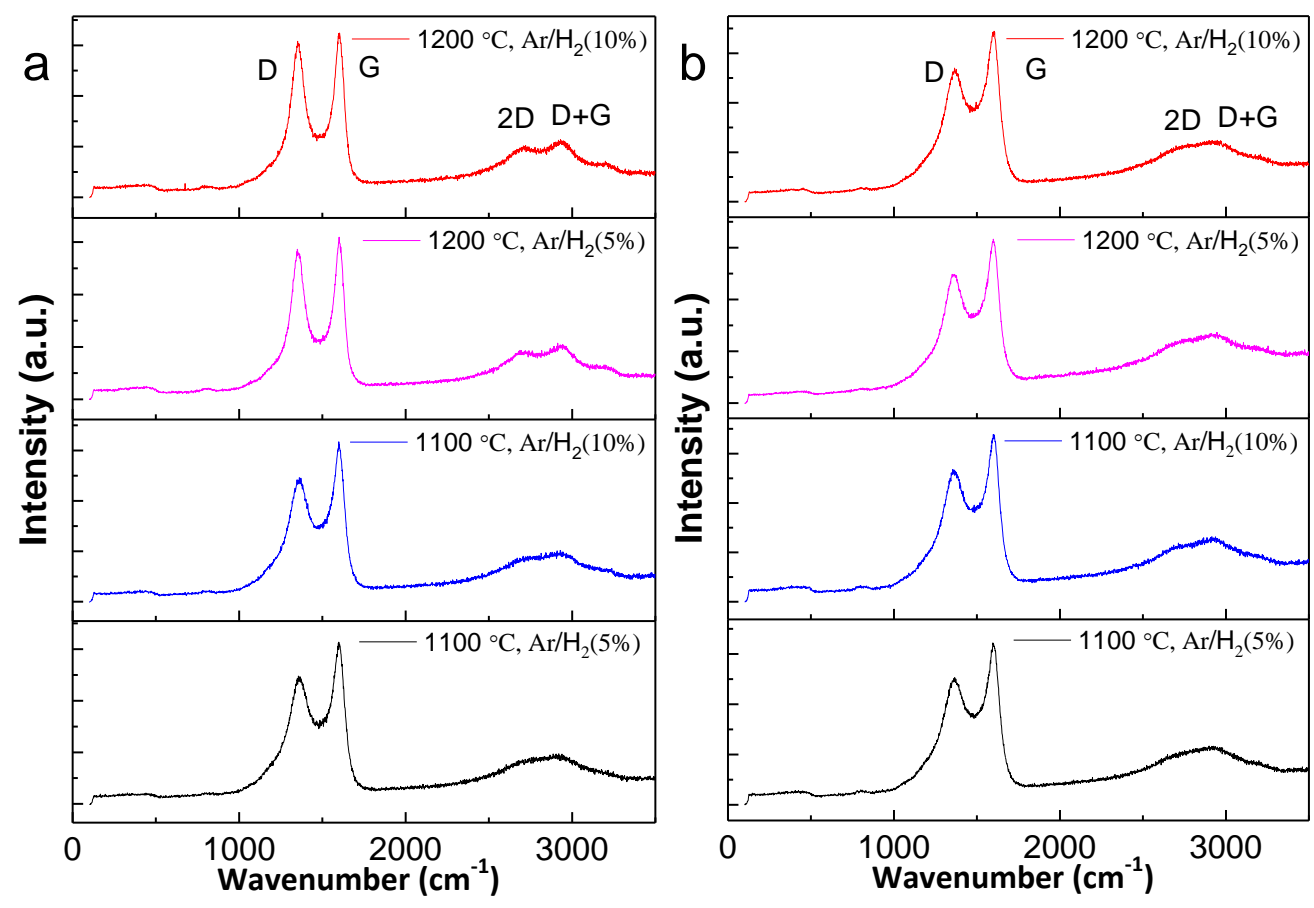

Figure 3.4. Raman spectra of (N)G prepared from chitosan (a) and G prepared from alginic acid(b) pyrolyzed at $1100,1200{ }^{\circ} \mathrm{C}$ under $\mathrm{Ar} / \mathrm{H}_{2}$ (5\%) or (10\%) flow, respectively.

The beneficial influence of the optimal pyrolysis temperature and the presence of $\mathrm{H}_{2}$ on the decrease on the oxygen content of the resulting films were also confirmed by XPS analysis. The carbon, oxygen and nitrogen content for each of the films prepared starting from alginic acidand chitosan under the various preparation conditions are summarized in Table 3.2.

As it can be seen in Table 3.2, the oxygen content determined from the atomic percentage measured by XPS depends on the nature of the biopolymer, being higher for chitosan than for alginic acid, and decreases gradually with the pyrolysis temperature up to the $1200^{\circ} \mathrm{C}$, reaching the lowest values of $6.64,6.92$ at $\%$, for $\mathrm{G}$ or (N)G, respectively, under a flow of $\mathrm{Ar} / \mathrm{H}_{2}$.

The presence of $\mathrm{H}_{2}$ during the pyrolysis produces defective graphene samples in 
where the oxygen content is consistently lower than that of the analogous samples prepared in the absence of $\mathrm{H}_{2}$. These analytical XPS data agree with the hypothesis that the $\mathrm{H}_{2}$ can react with the oxygen from the residual oxygenated functional groups during pyrolysis, resulting in the removal of some reactive oxygen atoms or functional groups from the resulting graphene samples. However, comparing with the samples obtained in the absence of $\mathrm{H}_{2}$, the difference in the oxygen content is very minor, indicating that only a small amount of oxygen is eliminated due to $\mathrm{H}_{2}$. Also, the carbon content shows a trend with the pyrolysis temperature and becomes slightly higher by introducing $\mathrm{H}_{2}$ during the process. Thus, the carbon content of 79.68 at\% from (N)G and 87.40 at\% from $\mathrm{G}$ achieved at a pyrolysis temperature of $900{ }^{\circ} \mathrm{C}$ under Ar increased gradually to 92.22 and 93.36 at\%, respectively, when the pyrolysis temperature was $1200{ }^{\circ} \mathrm{C}$ and $\mathrm{H}_{2}$ gas was present. As it is known, the carbon to oxygen atomic ratio ( $\mathrm{C} / \mathrm{O}$ ratio), which relates to the degree of oxidation, is one of the important factors determining the graphene quality. The highest $\mathrm{C} / \mathrm{O}$ ratio for (N)G and G obtained at a pyrolysis temperature of $1200{ }^{\circ} \mathrm{C}$ under $\mathrm{Ar} / \mathrm{H}_{2}$ is 13.33 and 14.06 , respectively. These $\mathrm{C} / \mathrm{O}$ ratios compared favorably with the values of 4.43 and 6.94 obtained at $900{ }^{\circ} \mathrm{C}$ under Ar.

It is worth noting that there was a jump of $\mathrm{C} / \mathrm{O}$ ratio in $\mathrm{G}$ from 6.94 to 10.24 when $\mathrm{H}_{2}$ was introduced into the system at a pyrolysis temperature of $900{ }^{\circ} \mathrm{C}$. It should also be noted that these values obtained at the high pyrolysis temperature of 1100 or $1200^{\circ} \mathrm{C}$, no matter whether or not $\mathrm{H}_{2}$ is present, are higher than the most of the $\mathrm{C} / \mathrm{O}$ ratios achieved for $\mathrm{rGO}$ prepared with different reduction methods. ${ }^{[20]}$

For $(\mathrm{N}) \mathrm{G}$, the presence of $\mathrm{H}_{2}$ resulted in a slightly decreased nitrogen content in the samples, having a similar influence on this heteroatom as the increase in the pyrolysis temperature. This probably reflects the higher graphitization of carbon in these conditions. The highest $\mathrm{N}$ doping level of 2.32 at\% was achieved at a pyrolysis temperature of $900{ }^{\circ} \mathrm{C}$ under $\mathrm{Ar}$ and then diminished to 2.06 at\% in the presence of $\mathrm{H}_{2}$, which finally decreased to 0.86 at\% at a pyrolysis temperature of $1200{ }^{\circ} \mathrm{C}$ under $\mathrm{Ar} / \mathrm{H}_{2}$. 
Tables 3.3 and 3.4 show the detailed bonding information of $\mathrm{C} 1 \mathrm{~s}$ for all the $\mathrm{G}$ samples as well as also that of $\mathrm{N}$ 1s for the (N)G. Some representative high-resolution XPS $C$ 1s peaks for (N)G and $G$ samples are presented in Figures 3.5 and 3.6 respectively, while some high-resolution XPS N1s peaks for (N)G samples are presented in Figure 3.7.

Table 3.2. Elemental composition of graphene films prepared from chitosan or alginic acidaccording to the XPS data.

\begin{tabular}{|c|c|c|c|c|c|c|c|c|c|}
\hline & & & \multicolumn{4}{|c|}{$(N) G$} & \multicolumn{3}{|c|}{ G } \\
\hline Entry & $\mathrm{T}\left({ }^{\circ} \mathrm{C}\right)$ & Gas & C (at\%) & O (at\%) & N (at\%) & $\mathrm{C} / \mathrm{O}$ ratio & C (at\%) & O (at\%) & $\mathrm{C} / \mathrm{O}$ ratio \\
\hline 1 & 900 & $\mathrm{Ar}$ & 79.68 & 18.00 & 2.32 & 4.43 & 87.40 & 12.60 & 6.94 \\
\hline 2 & 900 & $\mathrm{Ar} / \mathrm{H}_{2}$ & 84.49 & 13.46 & 2.06 & 6.28 & 91.10 & 8.90 & 10.24 \\
\hline 3 & 1000 & $\mathrm{Ar}$ & 87.79 & 10.17 & 2.03 & 8.631 & 91.50 & 8.50 & 10.76 \\
\hline 4 & 1000 & $\mathrm{Ar} / \mathrm{H}_{2}$ & 90.70 & 7.45 & 1.86 & 12.17 & 92.18 & 7.82 & 11.79 \\
\hline 5 & 1100 & $\mathrm{Ar}$ & 89.67 & 8.51 & 1.81 & 10.54 & 91.53 & 8.47 & 10.81 \\
\hline 6 & 1100 & $\mathrm{Ar} / \mathrm{H}_{2}$ & 91.55 & 7.09 & 1.35 & 12.91 & 92.26 & 7.74 & 11.92 \\
\hline 7 & 1200 & $\mathrm{Ar}$ & 91.41 & 7.44 & 1.16 & 12.29 & 92.06 & 8.05 & 11.59 \\
\hline 8 & 1200 & $\mathrm{Ar} / \mathrm{H}_{2}$ & 92.22 & 6.92 & 0.86 & 13.33 & 93.36 & 6.64 & 14.06 \\
\hline
\end{tabular}


Table 3.3. Distribution of $\mathrm{C}$ atoms among different chemical environments as determined by deconvolution of the high resolution XPS C 1s peak for all samples under study.

\begin{tabular}{|c|c|c|c|c|c|c|c|c|c|c|}
\hline \multicolumn{2}{|c|}{} & \multicolumn{5}{|c|}{$(N) G$} & \multicolumn{3}{c|}{} \\
\hline Entry & $T\left({ }^{\circ} \mathrm{C}\right)$ & Gas & $\mathrm{C}-\mathrm{C}$ & $\mathrm{C}-\mathrm{O} / \mathrm{C}-\mathrm{N}$ & $\mathrm{C}=\mathrm{O}$ & $\mathrm{C}(=\mathrm{O})-\mathrm{O}$ & $\mathrm{C}-\mathrm{C}$ & $\mathrm{C}-\mathrm{O}$ & $\mathrm{C}=\mathrm{O}$ & $\mathrm{C}(=\mathrm{O})-\mathrm{O}$ \\
\hline 1 & 900 & $\mathrm{Ar}$ & 66.36 & 26.33 & 5.33 & 1.99 & 63.91 & 25.73 & 6.20 & 4.16 \\
\hline 2 & 900 & $\mathrm{Ar} / \mathrm{H}_{2}$ & 67.46 & 23.57 & 5.06 & 3.92 & 66.25 & 22.95 & 5.20 & 5.60 \\
\hline 3 & 1000 & $\mathrm{Ar}$ & 67.17 & 23.46 & 4.09 & 5.28 & 67.10 & 22.23 & 5.85 & 4.82 \\
\hline 4 & 1000 & $\mathrm{Ar} / \mathrm{H} 2$ & 68.14 & 23.84 & 3.30 & 4.72 & 67.35 & 23.13 & 3.43 & 6.09 \\
\hline 5 & 1100 & $\mathrm{Ar}$ & 68.19 & 22.53 & 4.63 & 4.66 & 67.62 & 24.12 & 3.35 & 4.91 \\
\hline 6 & 1100 & $\mathrm{Ar} / \mathrm{H}_{2}$ & 68.60 & 22.81 & 2.81 & 5.78 & 67.67 & 22.63 & 4.23 & 5.47 \\
\hline 7 & 1200 & $\mathrm{Ar}$ & 68.91 & 23.68 & 3.67 & 3.74 & 68.44 & 22.05 & 3.83 & 5.67 \\
\hline 8 & 1200 & $\mathrm{Ar} / \mathrm{H}_{2}$ & 69.68 & 20.80 & 5.20 & 4.32 & 70.44 & 21.39 & 3.61 & 4.57 \\
\hline
\end{tabular}

In general, for the high-resolution XPS C 1s peak of all the graphene samples, the peaks can be deconvoluted into four individual components appearing at binding energy values $284.5,285.9,288.3$ and $290.2 \mathrm{eV}$, which should correspond to graphitic $\mathrm{C}, \mathrm{C}-\mathrm{O} / \mathrm{C}-\mathrm{N}, \mathrm{C}=\mathrm{O}$ and $\mathrm{C}(=\mathrm{O})-\mathrm{O}$, respectively. It seems that increasing pyrolysis temperature as well as the presence of $\mathrm{H}_{2}$ during process have a positive influence on the quality of $\mathrm{G}$ and $(\mathrm{N}) \mathrm{G}$ samples, resulting in the materials having slightly higher percentage of graphitic $\mathrm{C}$ and lower percentages of $\mathrm{C}-\mathrm{O} / \mathrm{C}-\mathrm{N}$ and $\mathrm{C}=\mathrm{O}$ bonds. Thus, it was observed that the $C 1$ s peak becomes narrower with these two parameters. As an example, (N)G prepared at $1200{ }^{\circ} \mathrm{C}$ under $\mathrm{Ar} / \mathrm{H}_{2}$ contains, according to the best fitting, a $69.68 \%$ of graphitic C, and a $20.80 \%$ of C-O/C-N, $5.20 \%$ of C=O and $4.32 \%$ of $\mathrm{C}(=\mathrm{O})-\mathrm{O}$. In comparison, $(\mathrm{N}) \mathrm{G}$ obtained at $900^{\circ} \mathrm{C}$ under $\mathrm{Ar}$ contains a $66.36 \%$ of 
graphitic $\mathrm{C}$, and percentages of $26.33,5.33$ and $1.99 \%$ of $\mathrm{C}-\mathrm{O} / \mathrm{C}-\mathrm{N}, \mathrm{C}=\mathrm{O}$ and $\mathrm{C}(=\mathrm{O})-\mathrm{O}$, respectively.

Table 3.4. Distribution of $\mathrm{N}$ atoms among different chemical environments as determined by deconvolution of the high resolution XPS N 1s peak for the (N)G samples under study.

\begin{tabular}{|c|c|c|c|c|c|}
\hline \multicolumn{2}{|l}{} & \multicolumn{3}{|c|}{$(\mathrm{N}) \mathrm{G}$} \\
\hline Entry & $\mathrm{T}\left({ }^{\circ} \mathrm{C}\right)$ & Gas & Graphitic & Pyrrolic & Pyridinic \\
\hline 1 & 900 & $\mathrm{Ar}$ & 17.21 & 47.37 & 35.43 \\
\hline 2 & 900 & $\mathrm{Ar} / \mathrm{H}_{2}$ & 19.82 & 51.63 & 28.55 \\
\hline 3 & 1000 & $\mathrm{Ar}$ & 20.82 & 50.31 & 28.87 \\
\hline 4 & 1000 & $\mathrm{Ar} / \mathrm{H}_{2}$ & 22.76 & 51.26 & 25.98 \\
\hline 5 & 1100 & $\mathrm{Ar}$ & 23.05 & 55.18 & 21.77 \\
\hline 6 & 1100 & $\mathrm{Ar} / \mathrm{H}_{2}$ & 26.73 & 57.08 & 16.18 \\
\hline 7 & 1200 & $\mathrm{Ar}$ & 36.93 & 41.44 & 21.63 \\
\hline 8 & 1200 & $\mathrm{Ar} / \mathrm{H}_{2}$ & 38.24 & 40.80 & 20.95 \\
\hline
\end{tabular}

In contrast for the (N)G sample prepared at $900{ }^{\circ} \mathrm{C}$ under $\mathrm{Ar}$, the XPS $\mathrm{N} 1 \mathrm{~s}$ peak shown in Figure 3.5 can be fitted to three main peaks, centered at 398.5, 400.6, $401.6 \mathrm{eV}$, corresponding to pyridinic, pyrrolic and graphitic $\mathrm{N}$, respectively. The contents of these three $\mathrm{N}$ components are $35.43,47.37$ and $19.21 \%$, respectively. This peak analysis indicates that the prevalent families of $\mathrm{N}$ atoms incorporated into the graphene are pyridinic and pyrrolic $\mathrm{N}$. 

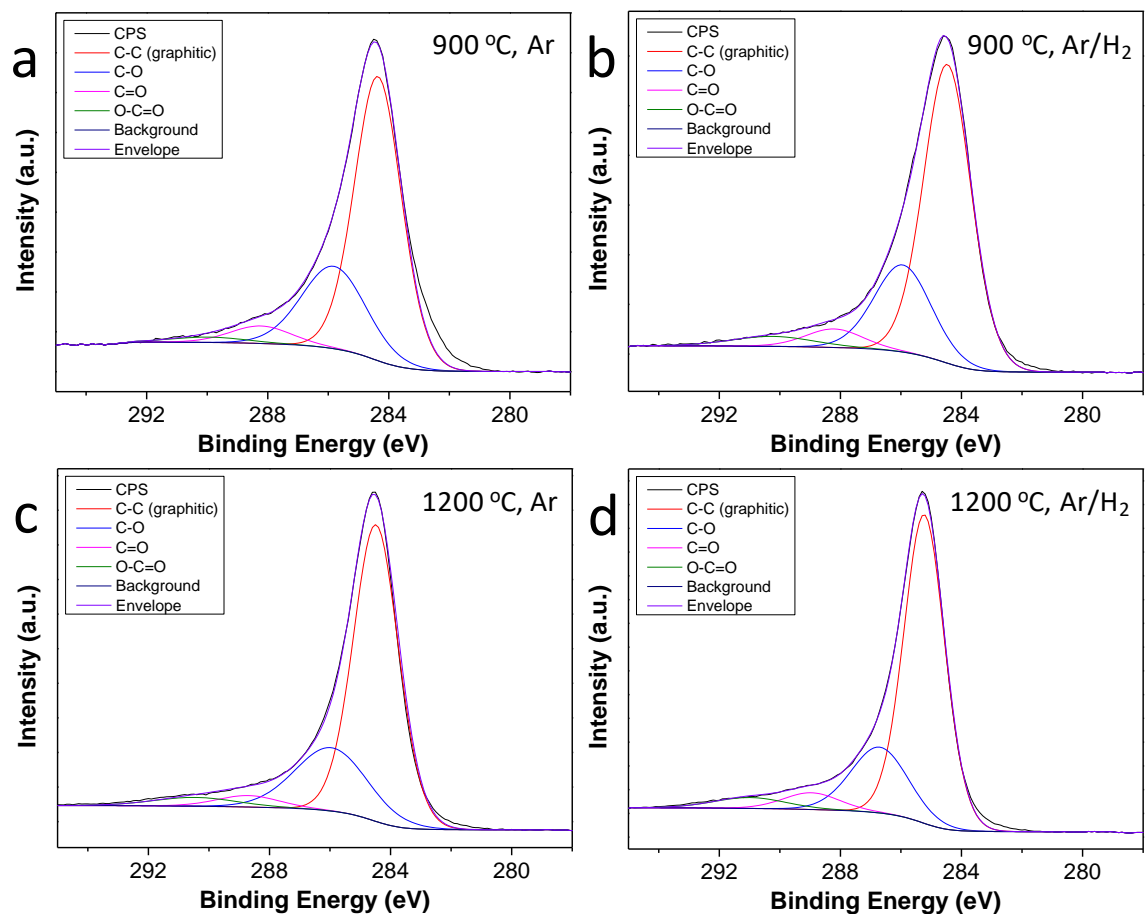

Figure 3.5. High resolution XPS C 1 s peak of $(\mathrm{N}) \mathrm{G}$ pyrolyzed at $900{ }^{\circ} \mathrm{C}$ under $\operatorname{Ar}(\mathrm{a})$ or $\mathrm{Ar} / \mathrm{H}_{2}(5 \%)$ (b) and pyrolyzed at $1200^{\circ} \mathrm{C}$ under $\mathrm{Ar}$ (c) or $\mathrm{Ar} / \mathrm{H}_{2}(5 \%)$ (d).
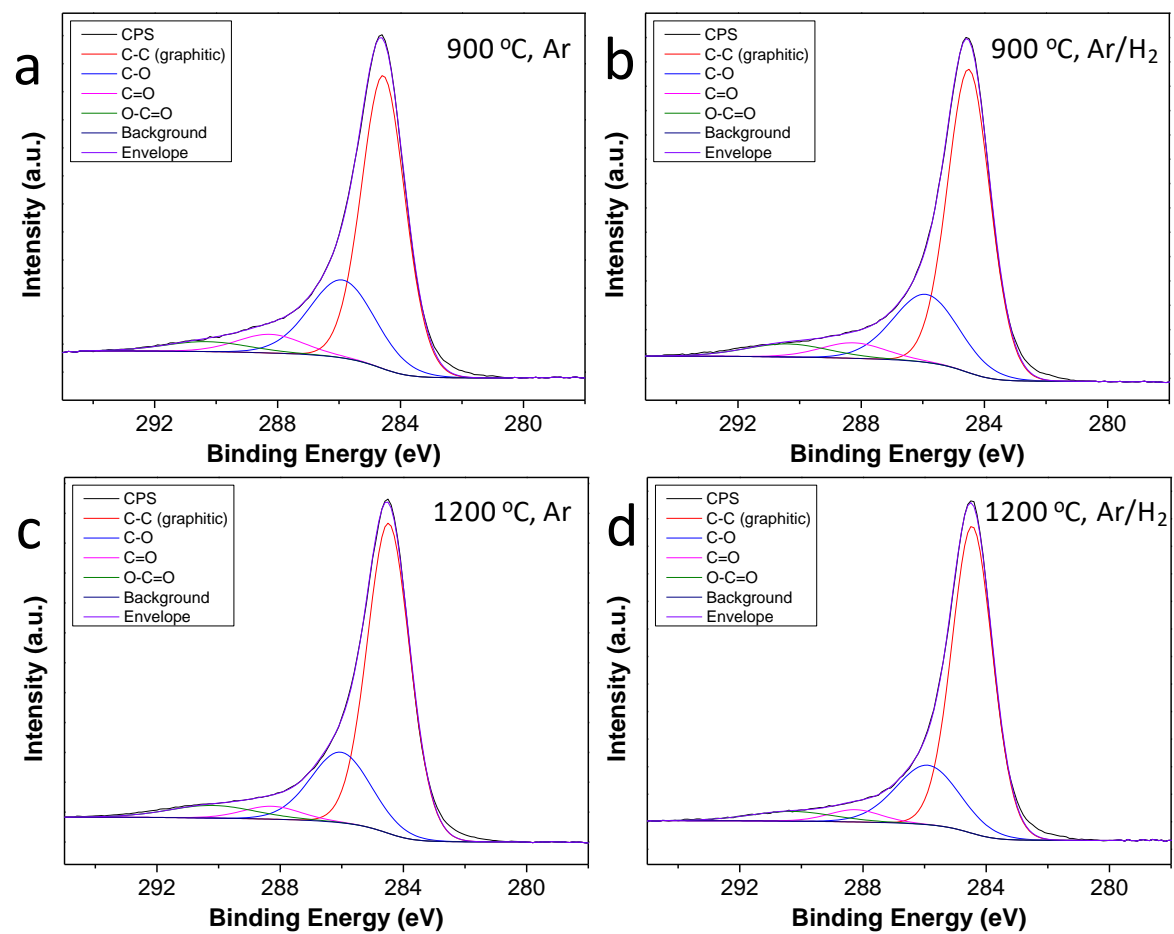

Figure 3.6. High resolution XPS C1s peak of $\mathrm{G}$ pyrolyzed at $900{ }^{\circ} \mathrm{C}$ under $\mathrm{Ar}$ (a) or $\operatorname{Ar} / \mathrm{H}_{2}(5 \%)$ (b) and $\mathrm{G}$ pyrolyzed at $1200{ }^{\circ} \mathrm{C}$ under $\operatorname{Ar}(\mathbf{c})$ or $\mathrm{Ar} / \mathrm{H}_{2}(5 \%)$ (d). 

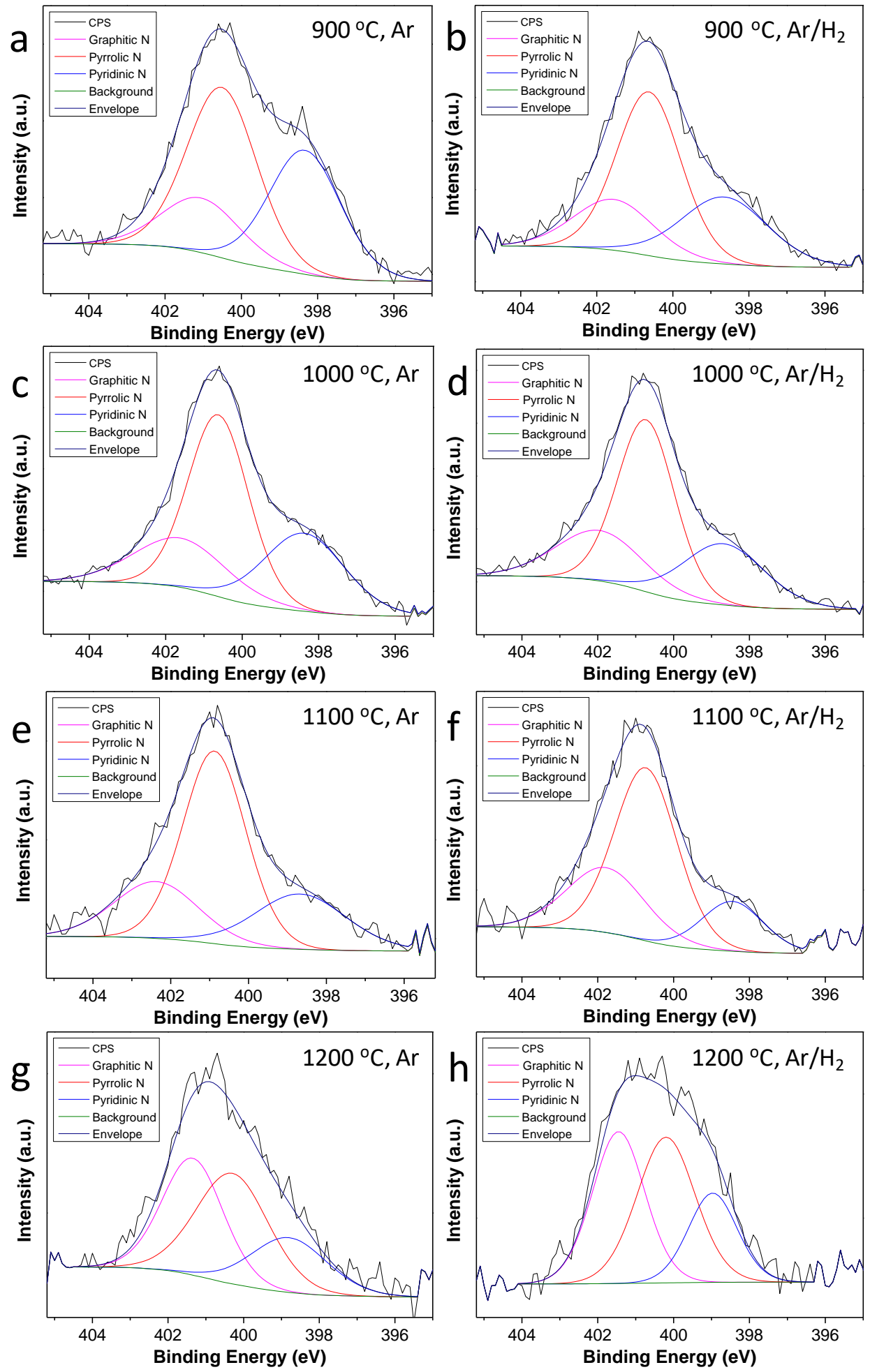

Figure 3.7. High resolution XPS $N$ 1s peak of $(N) G$ prepared at the pyrolysis temperature of $900,1000,1100,1200{ }^{\circ} \mathrm{C}$ under $\mathrm{Ar}$ (left side) or $\mathrm{Ar} / \mathrm{H}_{2}(5 \%$ ) (right side), respectively. 
As it can be seen in Table 3.4 and Figure 3.7, with the increase of pyrolysis temperature, the general tendency observed is a gradual decrease in the percentage of pyridinic $\mathrm{N}$, while the proportion of pyrrolic $\mathrm{N}$ is maintained and the contribution of graphitic $\mathrm{N}$ increases. This trend indicates that the thermodynamically more stable form of $\mathrm{N}$ atoms in (N)G is graphitic $\mathrm{N}$, while that of pyridinic $\mathrm{N}$ appears to be the weakest, disappearing as the pyrolysis temperature increases. On the other hand, following a similar trend with the influence of pyrolysis temperature, the presence of $\mathrm{H}_{2}$ slightly decreases the $\mathrm{N}$ content and at the same time changes also the distribution of this element among the three major $\mathrm{N}$ families, increasing the contribution of graphitic $\mathrm{N}$ in the (N)G samples.

Since, as just commented, Raman spectroscopy and XPS analysis can report on the quality of the defective graphene samples as a function of the preparation conditions, based on the density of defects and oxygen content, additional experiments were carried out at $1100{ }^{\circ} \mathrm{C}$ using $10 \% \mathrm{H}_{2}$ in the gas flow during the pyrolysis step. As indicated above, it seems that, in the presence of $H_{2}$, the differences in the samples pyrolyzed at 1100 or $1200{ }^{\circ} \mathrm{C}$ are minimal, although the films become thinner when the concentration of $\mathrm{H}_{2}$ increased. It was observed that the samples prepared at $1100{ }^{\circ} \mathrm{C}$ under $10 \% \mathrm{H}_{2}$ have almost identical Raman spectra and XPS data with the analogous samples prepared with $5 \% \mathrm{H}_{2}$. These data indicate that $5 \%$ of $\mathrm{H}_{2}$ content during the pyrolysis step meets a compromise between better quality films and low $\mathrm{H}_{2}$ consumption.

The morphology of the defective films was studied by SEM and TEM and the thickness was determined by AFM. No obvious differences among the various films as a function of the pyrolysis temperature in the range of $900-1200{ }^{\circ} \mathrm{C}$ or prepared in the absence and presence of $5 \% \mathrm{H}_{2}$ were observed by SEM. Nevertheless, the films prepared from chitosan appeared consistently in SEM with much lesser roughness than those from alginic acid. Perusal of the TEM images clearly indicates that the degree of ordering and graphitization of the films prepared at $1200{ }^{\circ} \mathrm{C}$ in the presence of $\mathrm{H}_{2}$ was higher than those obtained at $900{ }^{\circ} \mathrm{C}$ in its absence. Figure 3.8 
shows representative TEM images and Fourier-transformed electron diffraction pattern to illustrate the improvement in the crystallinity of the sheets upon optimization of the preparation conditions.
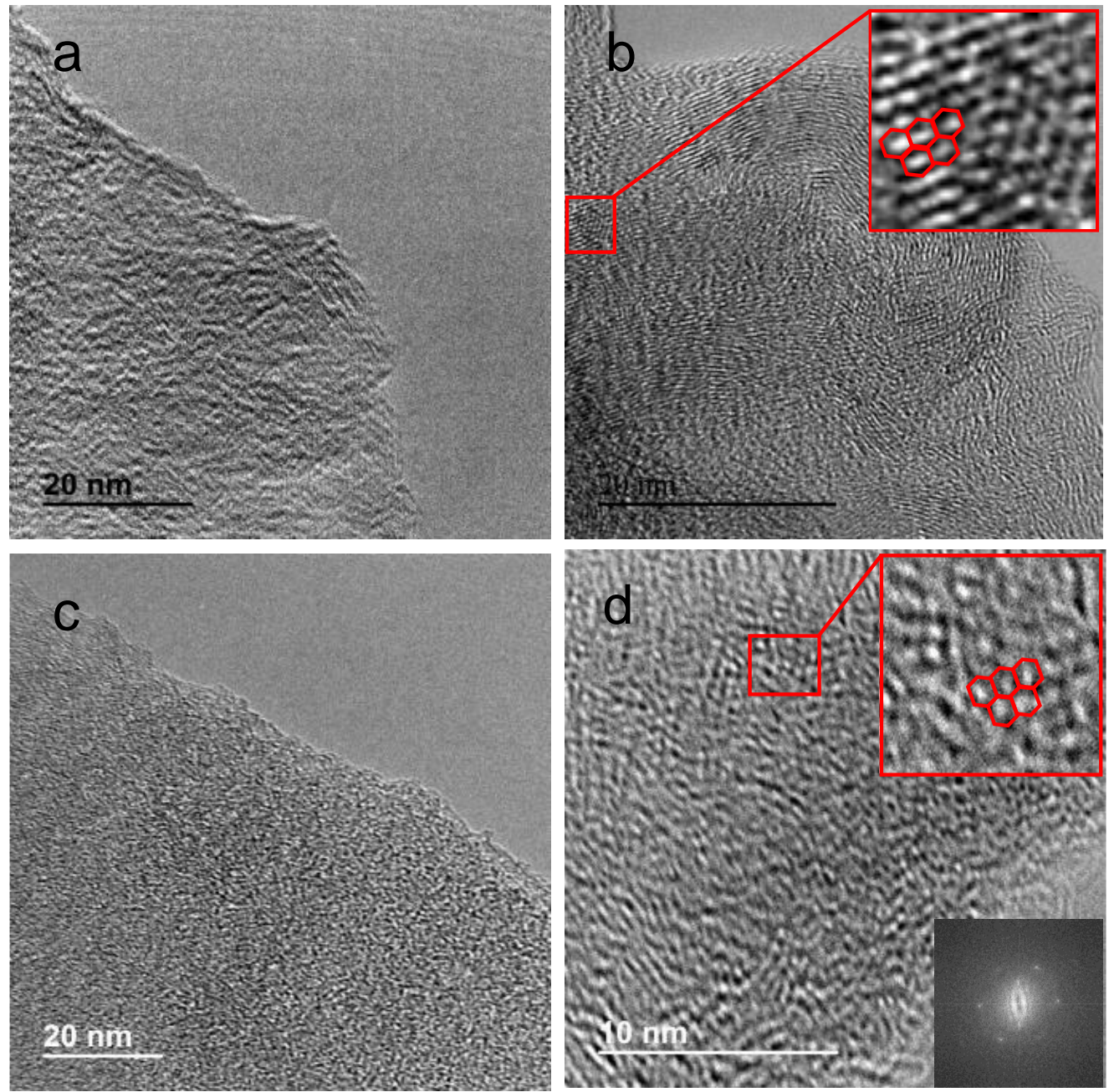

Figure 3.8. TEM images of (N)G pyrolyzed at $900{ }^{\circ} \mathrm{C}$ under $\mathrm{Ar}(\mathrm{a})$ or at $1200{ }^{\circ} \mathrm{C}$ under $\mathrm{Ar} / \mathrm{H}_{2}(5 \%)$ (b) and $\mathrm{G}$ pyrolyzed at $900{ }^{\circ} \mathrm{C}$ under $\mathrm{Ar}(\mathbf{c})$ or at $1200{ }^{\circ} \mathrm{C}$ under $\mathrm{Ar} / \mathrm{H}_{2}(5 \%)$ (d). The hexagonal arrangement observed in the high-resolution images has been highlighted in red. The inset shows the Fourier-transformed electron diffraction pattern of image $\mathbf{d}$.

AFM measurements indicate that starting from alginic acid or chitosan films of the same thickness, the resulting graphene film becomes thinner upon increasing the pyrolysis temperature and when $\mathrm{H}_{2}$ was present in the atmosphere during the 
process. As an example, Figure 3.9 presents representative AFM images and the corresponding film thicknesses for two (N)G films prepared under different conditions.
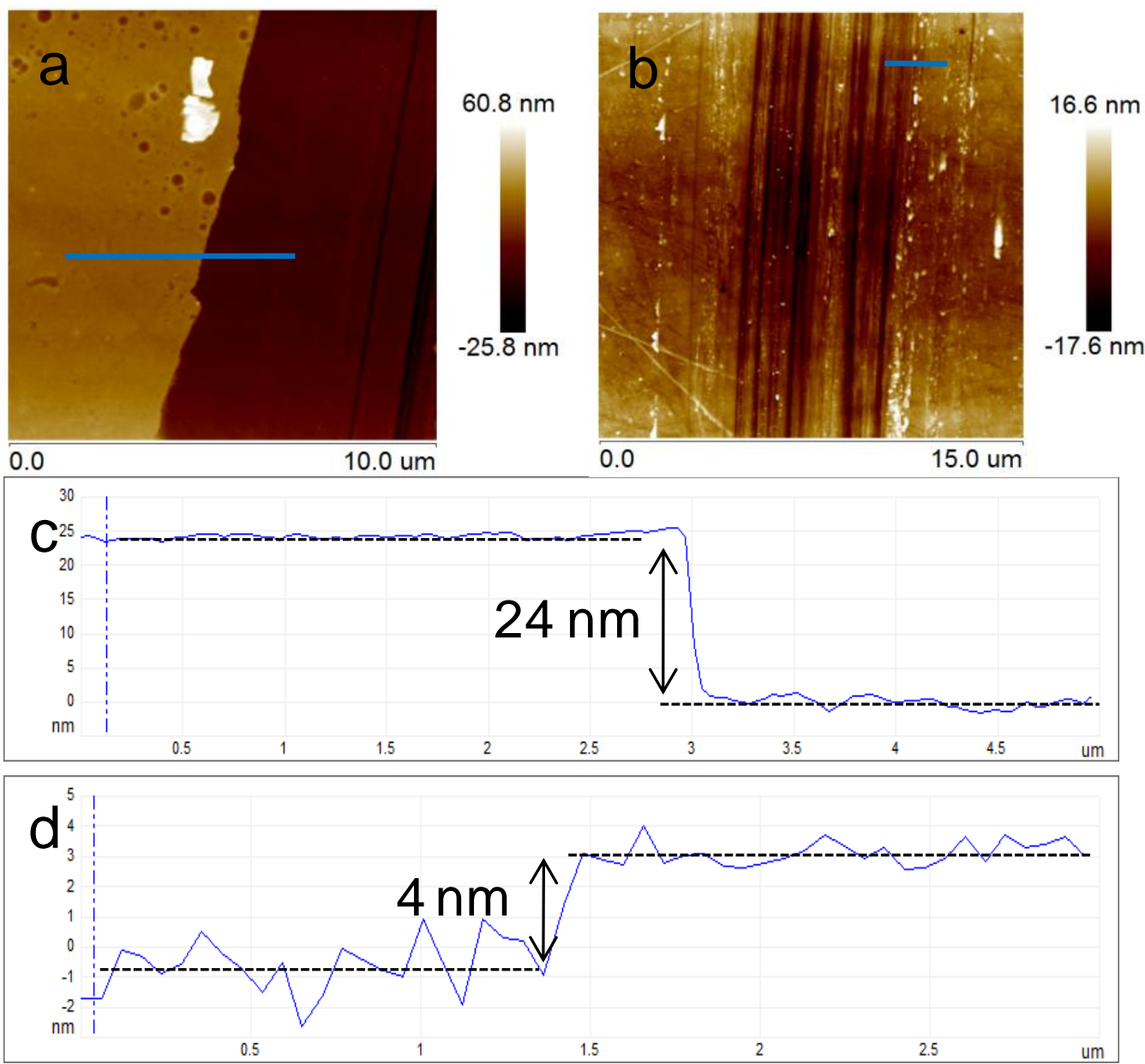

Figure 3.9. AFM images of two (N)G films prepared from chitosan pyrolyzed at 1100 (a) and $1200{ }^{\circ} \mathrm{C}$ under $\mathrm{Ar} / \mathrm{H}_{2}(5 \%)$ flow (b). Images (c, d) show the section profiles of images $\mathbf{a}$ and $\mathbf{b}$, respectively, along the blue lines indicated in the frames $\mathbf{a}$ and $\mathbf{b}$. Thicknesses about 24 and $4 \mathrm{~nm}$ were determined for the films prepared at 1100 and $1200{ }^{\circ} \mathrm{C}$, respectively.

As commented at the beginning of the Chapter, the purpose of the present study was to determine if there is an increase in the conductivity of the graphene films upon optimization of the preparation conditions and, particularly, by optimization of the pyrolysis temperature and introduction of $\mathrm{H}_{2}$ in the gas flow. The 
results of the conductivity measurement are presented in Figure 3.10, where plots of the electrical conductivity measured using a four-tips head vs. the pyrolysis temperature in the absence and presence of $\mathrm{H}_{2}$ are presented.

As one can see in this Figure 3.10, the electrical resistance of the defective graphene films prepared from alginic acidand chitosan follows a similar trend with the pyrolysis temperature and in the presence of $\mathrm{H}_{2}$ although it was somewhat more remarkable in the case of chitosan.

The electrical resistance of the defective graphene films decreases with the temperature from 900 to $1100{ }^{\circ} \mathrm{C}$ and it increases beyond this temperature. The presence of $5 \% \mathrm{H}_{2}$ during the pyrolysis has always a beneficial influence decreasing the electrical resistance at all temperatures and for both precursors. By optimization of the pyrolysis conditions, the electrical resistance could be decreased by a factor of about 3 and 5 for alginic acidand chitosan, respectively. The minimum electrical resistance measured in the present study was 1000 and $1100 \Omega /$ sq for defective films from alginic acidand chitosan, respectively.
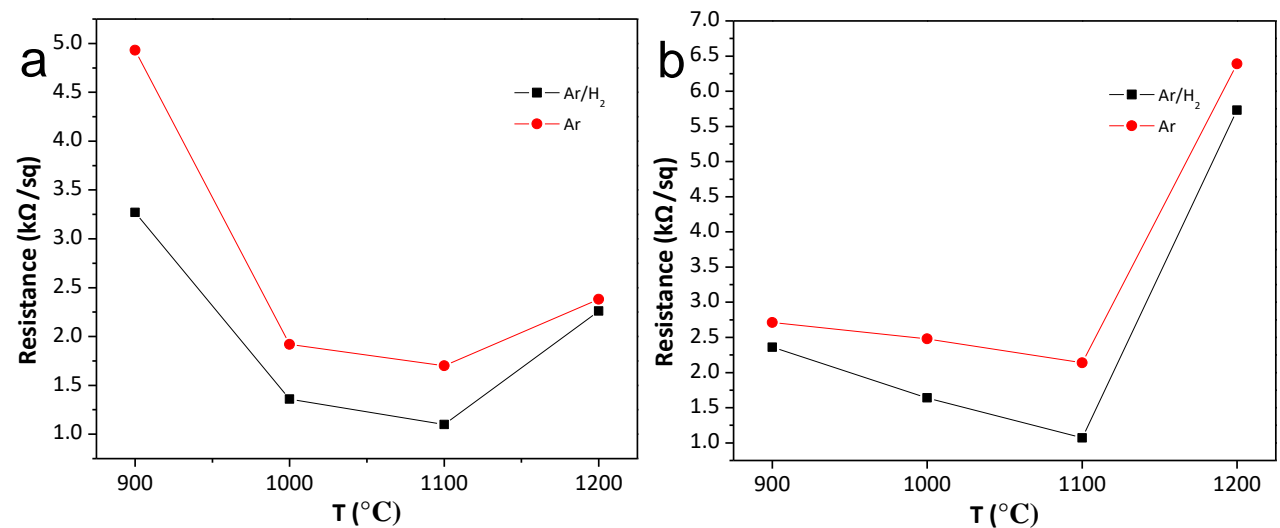

Figure 3.10. Plots of the electrical resistance for $(\mathrm{N}) \mathrm{G}(\mathrm{a})$ and $\mathrm{G}(\mathrm{b})$ films on quartz substrate prepared at different pyrolysis temperatures under $\operatorname{Ar}$ or $\mathrm{Ar} / \mathrm{H}_{2}(5 \%)$ flow.

The most probable reason why the electrical resistance increases at high temperature is the inappropriateness of the four-tips head to measure the electrical 
conductivity of the thinnest films of just a few nanometers thickness that are obtained at $1200{ }^{\circ} \mathrm{C}$. It may also happen that these films are also not totally continuous at the millimetric length scale needed for the electrical resistance measurements due to volatilization of the biopolymer.

The (N)G and $G$ films were used as photoelctrodes for hydrogen evolutionreaction. The similar test was carried out in previous work from the group, using ( $\mathrm{N}$ ) G film as the working electrode and aqueous $\mathrm{Na}_{2} \mathrm{~S} / \mathrm{Na}_{2} \mathrm{SO}_{3}$ solution as electrolyte under illumination of a UV/Vis $300 \mathrm{~W}$ Xe lamp. ${ }^{[21]}$ In the present work, line-sweep voltammgrams (LSV) were performed with the graphene electrodes at a

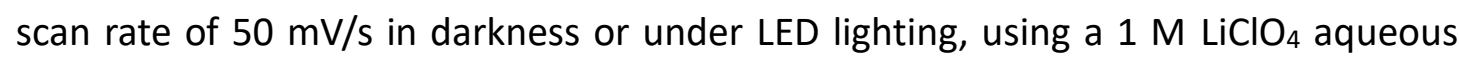
solution as electrolyte. The results are shown in Figure 3.11. As we can see, for both (N)G and $\mathrm{G}$, the film prepared at $1100{ }^{\circ} \mathrm{C}, \mathrm{Ar} / \mathrm{H}_{2}$ flow shows a smaller difference in the current density between light and dark, comparing with the film prepared at 900 ${ }^{\circ} \mathrm{C}$ under $\mathrm{Ar} / \mathrm{H}_{2}$ or Ar flow. Since the defective graphenes with bandgap are able to photogenerate charges under light irradiation, making the current a little higher than the value in darkness, the degree of the difference between these two curves can somewhat indicate the defective level of the films. These results from the experimental samples demonstrate that the film prepared at higher pyrolysis temperature and in the presence of $\mathrm{H}_{2}$ shows a more perfect graphene structure with lower density of defects, which is in good agreement with the characterization results from Raman, XPS, TEM. 

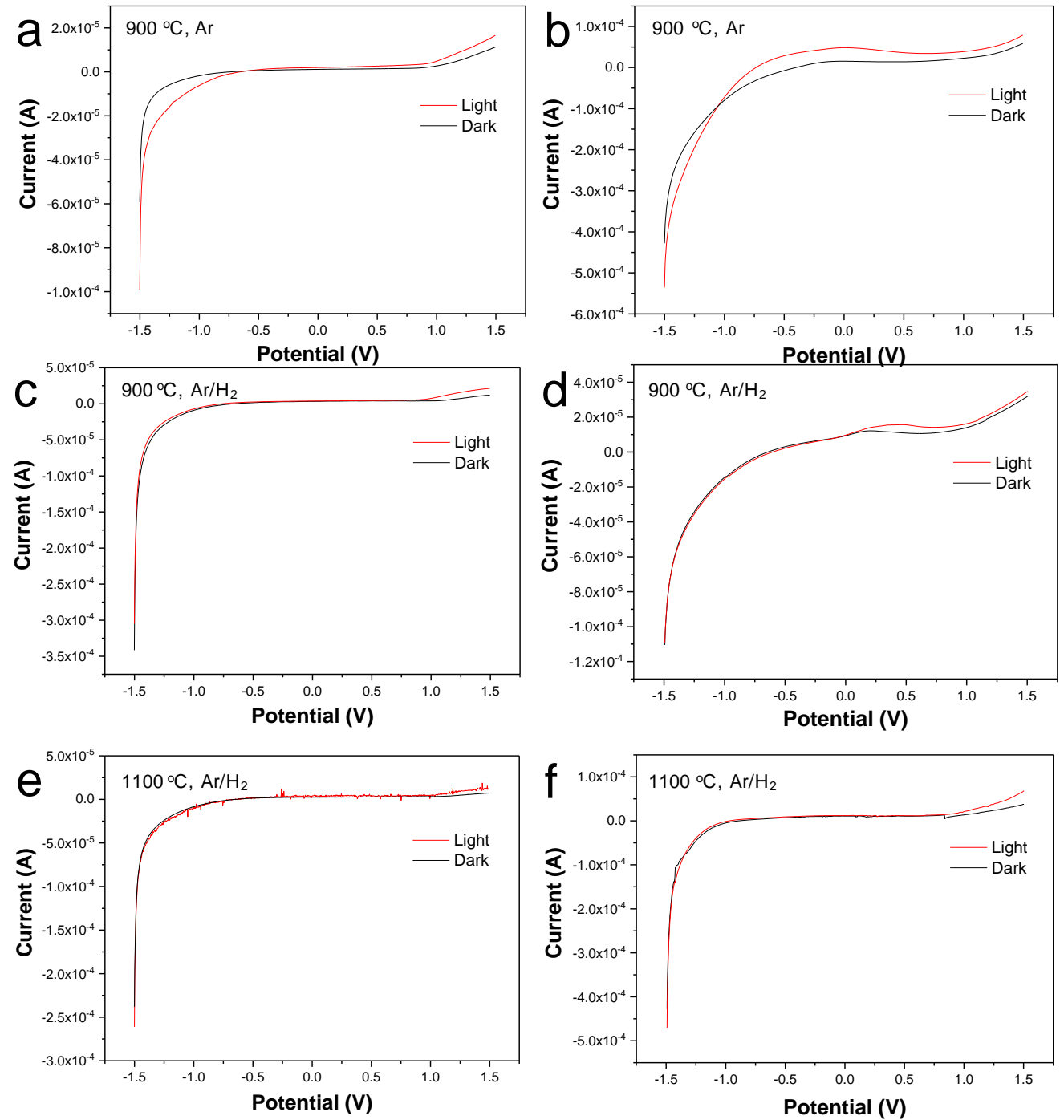

Figure 3.11. LSV curves measured at a scan rate of $50 \mathrm{mV} / \mathrm{s}$ in darkness or under LED illumination from the electrodes made of $(\mathrm{N}) \mathrm{G}$ prepared at $900{ }^{\circ} \mathrm{C}$ under $\mathrm{Ar}(\mathrm{a})$ or $\mathrm{Ar} / \mathrm{H}_{2}(5 \%)(\mathbf{c})$, at $1100{ }^{\circ} \mathrm{C}$ under $\mathrm{Ar} / \mathrm{H}_{2}(5 \%)(\mathrm{e})$ or $\mathrm{G}$ prepared at $900{ }^{\circ} \mathrm{C}$ under $\mathrm{Ar}(\mathbf{b})$ or $\mathrm{Ar} / \mathrm{H}_{2}(5 \%)(\mathbf{d})$, at $1100{ }^{\circ} \mathrm{C}$ under $\mathrm{Ar} / \mathrm{H}_{2}(5 \%)(\mathbf{f})$, respectively.

Also, the production of $\mathrm{H}_{2}$ under light or dark condition was measured during the photoelectric test with an applied bias of $+1.1 \mathrm{~V}$. The results are presented in Figure 3.12. As it can be seen, $G$ films exhibit better photocatalytic activity than (N)G films, although the activities of both two types of films are very low, considering the composition of the carbonaceous materials and the absence of any sacrificial reagent. The most perfect film, G film prepared at $1100{ }^{\circ} \mathrm{C}$ under $\mathrm{Ar} / \mathrm{H}_{2}$, shows the best 
catalytic activity, achieving a $\mathrm{H}_{2}$ production of $3.62 \mu \mathrm{mol}$ after $16 \mathrm{~h}$ under LED illumination, while the values for $\mathrm{G}$ films pyrolyzed at $900{ }^{\circ} \mathrm{C}$ under $\mathrm{Ar}$ or $\mathrm{Ar} / \mathrm{H}_{2}$ condition are 0.75 and $1.91 \mu \mathrm{mol}$ respectively. Control experiment for the $\mathrm{G}$ film prepared at $1100{ }^{\circ} \mathrm{C}$ under $\mathrm{Ar} / \mathrm{H}_{2}$ was performed under dark condition and the sample exhibited lower activity, with a $\mathrm{H}_{2}$ yield of $1.44 \mu \mathrm{mol}$. In the case of $\mathrm{N}$-doped graphene, although the activity for the experimental films is very low even under LED light irradiation, it still could be seen that the film prepared at $1100{ }^{\circ} \mathrm{C}$ under $\mathrm{Ar} / \mathrm{H}_{2}$ shows slightly higher activity than the film prepared at $900{ }^{\circ} \mathrm{C}$ under Ar. The more efficient performance of the graphene film should be due to the decrease in the intensity of defects on the structure, resulting from the higher pyrolysis temperature and the presence of $\mathrm{H}_{2}$. Similar with the photoelectrocatalysis based on other typical semiconductors, ${ }^{[22]}$ a proposed mechanism for the reaction using the graphene film as electrode is presented in Scheme 3.3. The electrode made of the graphene film is supposed to act as photoanode, where the photogenerated charges can be separated efficiently with the external bias of $+1.1 \mathrm{~V}$.

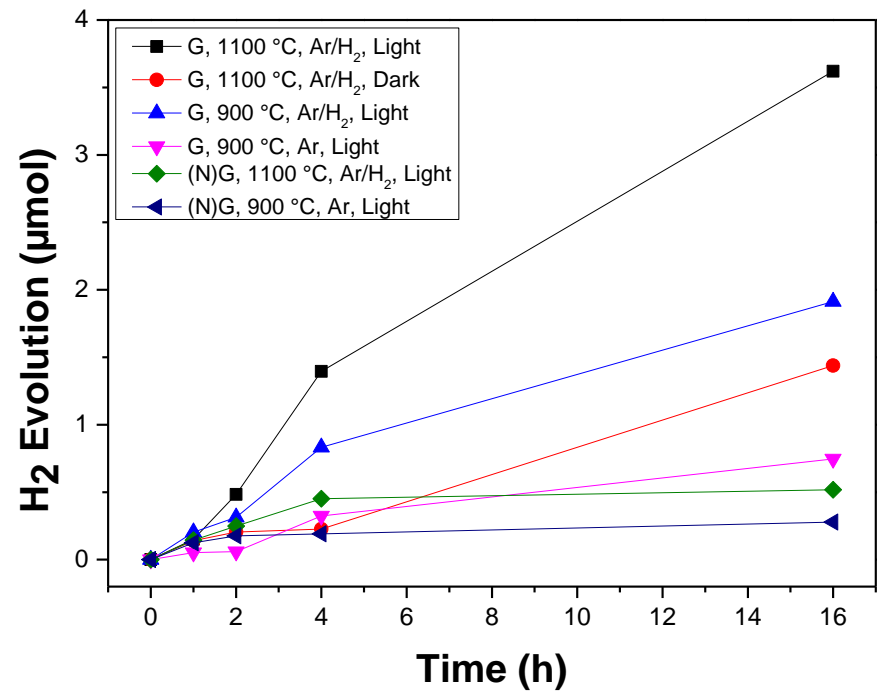

Figure 3.12. $\mathrm{H}_{2}$ evolutionobtained in darkness or under LED illumination using the electrodes made of (N)G or G films prepared at 900 or $1100{ }^{\circ} \mathrm{C}$ under $\operatorname{Ar}$ or $\mathrm{Ar} / \mathrm{H}_{2}(5 \%)$ flow. 


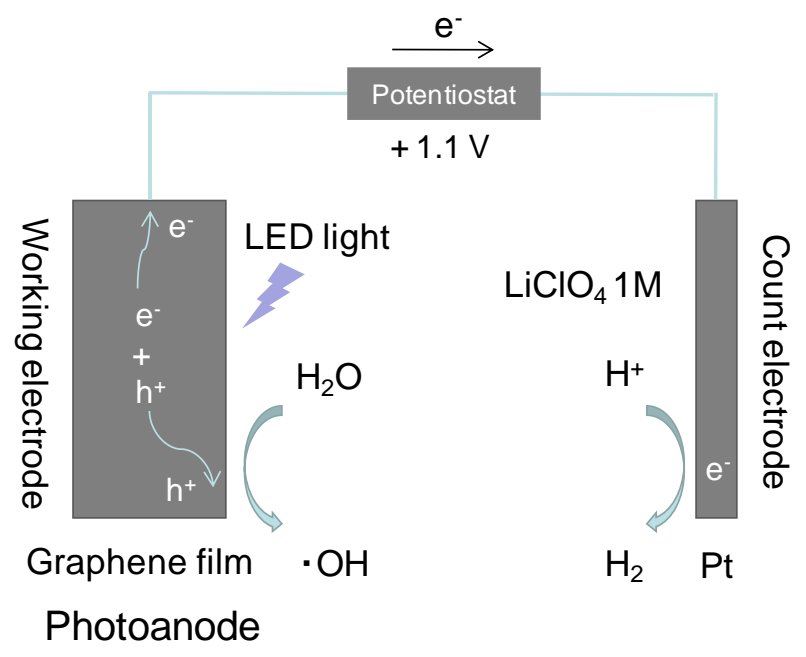

Scheme 3.3. A schematic representation of the possible mechanism of photoelectrocatalysis for the graphene film used as electrode under LED illumination with a positive $1.1 \mathrm{~V}$ applied bias. The test was performed using an aqueous solution of $1 \mathrm{M} \mathrm{LiClO}_{4}$ as electrolyte, a Pt foil as counter electrode and $\mathrm{Ag} / \mathrm{AgCl}$ as reference electrode.

\subsection{Conclusions}

The present Chapter has shown that the presence of a small percentage of $\mathrm{H}_{2}$ during the pyrolysis of natural filmogenic polysaccharides decreases the density of defects on the resulting undoped and doped graphenes, although it was not possible to remove completely the presence of oxygen in the samples, even at the highest pyrolysis temperatures. This incomplete decrease of the oxygen functionalities is reflected in an improvement of the electrical conductivity of the samples that can reach a value of $1000 \Omega / \mathrm{sq}$ by pyrolysing alginic acidat $1100{ }^{\circ} \mathrm{C}$ under $5 \% \mathrm{H}_{2}$. The electrode made of less defective graphene film exhibits photoelectrocatalytic activity for $\mathrm{H}_{2}$ evolutionat a positive applied bias under LED illumination. In the context of valorization of biomass waste, the present study shows that the properties of graphenes obtained from these residues can be easily improved by adequate 
optimization of the pyrolysis conditions, the challenge of complete oxygen removal still remaining. 


\subsection{References}

[1] Bonaccorso, F.; Lombardo, A.; Hasan, T.; Sun, Z. P.; Colombo, L.; Ferrari, A. C. Production and processing of graphene and 2D crystals. Materials Today 2012, 15(12), 564-589.

[2] Chen, D.; Feng, H. B.; Li, J. H. Graphene oxide: preparation, functionalization, and electrochemical applications. Chemical Reviews 2012, 112(11), 6027-6053.

[3] Luo, B.; Liu, S. M.; Zhi, L. J. Chemical approaches toward graphene-based nanomaterials and their applications in energy-related areas. Small 2012, 8(5), 630-646.

[4] Machado, B. F.; Serp, P. Graphene-based materials for catalysis. Catalysis Science \& Technology 2012, 2(1), 54-75.

[5] Lavorato, C.; Primo, A.; Molinari, R.; Garcia, H. Natural alginate as a graphene precursor and template in the synthesis of nanoparticulate ceria/graphene water oxidation photocatalysts. Acs Catalysis 2014, 4(2), 497-504.

[6] Primo, A.; Atienzar, P.; Sanchez, E.; Delgado, J. M.; Garcia, H. From biomass wastes to large-area, high-quality, $\mathrm{N}$-doped graphene: catalyst-free carbonization of chitosan coatings on arbitrary substrates. Chemical Communications 2012, 48(74), 9254-9256.

[7] Primo, A.; Sanchez, E.; Delgado, J. M.; Garcia, H. High-yield production of $\mathrm{N}$-doped graphitic platelets by aqueous exfoliation of pyrolyzed chitosan. Carbon 2014, 68, 777-783.

[8] Dhakshinamoorthy, A.; Latorre-Sanchez, M.; Asiri, A. M.; Primo, A.; Garcia, H. Sulphur-doped graphene as metal-free carbocatalysts for the solventless aerobic oxidation of styrenes. Catalysis Communications 2015, 65, 10-13.

[9] Dhakshinamoorthy, A.; Primo, A.; Concepcion, P.; Alvaro, M.; Garcia, H. Doped graphene as a metal-free carbocatalyst for the selective aerobic oxidation of benzylic hydrocarbons, cyclooctane and styrene. Chemistry-A European Journal 2013, 19(23), 7547-7554. 
[10] Latorre-Sanchez, M.; Primo, A.; Atienzar, P.; Forneli, A.; Garcia, H. p-n heterojunction of doped graphene films obtained by pyrolysis of biomass precursors. Small 2015, 11(8), 970-975.

[11] Primo, A.; Esteve-Adell, I.; Blandez, J. F.; Dhakshinamoorthy, A.; Alvaro, M.; Candu, N.; Coman, S. M.; Parvulescu, V. I.; Garcia, H. High catalytic activity of oriented 2.0.0 copper(I) oxide grown on graphene film. Nature Communications 2015, 6.

[12] Primo, A.; Esteve-Adell, I.; Coman, S. N.; Candu, N.; Parvulescu, V. I.; Garcia, H. One-step pyrolysis preparation of 1.1 .1 oriented gold nanoplatelets supported on graphene and six orders of magnitude enhancement of the resulting catalytic activity. Angewandte Chemie-International Edition 2016, 55(2), 607-612.

[13] Li, X.; Zhu, Y.; Cai, W.; Borysiak, M.; Han, B.; Chen, D.; Piner, R. D.; Colombo, L.; Ruoff, R. S. Transfer of large-area graphene films for high-performance transparent conductive electrodes. Nano letters 2009, 9(12), 4359-4363.

[14] Kim, K. S.; Zhao, Y.; Jang, H.; Lee, S. Y.; Kim, J. M.; Kim, K. S.; Ahn, J. H.; Kim, P.; Choi, J. Y.; Hong, B. H. Large-scale pattern growth of graphene films for stretchable transparent electrodes. Nature 2009, 457(7230), 706-710.

[15] Reina, A.; Jia, X.; Ho, J.; Nezich, D.; Son, H.; Bulovic, V.; Dresselhaus, M. S.; Kong, J. Large area, few-layer graphene films on arbitrary substrates by chemical vapor deposition. Nano letters 2008, 9(1), 30-35.

[16] Li, X.; Cai, W.; An, J.; Kim, S.; Nah, J.; Yang, D.; Piner, R.; Velamakanni, A.; Jung, I.; Tutuc, E. Large-area synthesis of high-quality and uniform graphene films on copper foils. Science 2009, 324(5932), 1312-1314.

[17] Cançado, L. G.; Jorio, A.; Martins Ferreira, E.; Stavale, F.; Achete, C.; Capaz, R.; Moutinho, M.; Lombardo, A.; Kulmala, T.; Ferrari, A. Quantifying defects in graphene via Raman spectroscopy at different excitation energies. Nano letters 2011, 11(8), 3190-3196.

[18] Eckmann, A.; Felten, A.; Mishchenko, A.; Britnell, L.; Krupke, R.; Novoselov, K. S.; Casiraghi, C. Probing the nature of defects in graphene by Raman spectroscopy. Nano letters 2012, 12(8), 3925-3930. 
[19] Ferrari, A. C.; Basko, D. M. Raman spectroscopy as a versatile tool for studying the properties of graphene. Nature nanotechnology 2013, 8(4), 235-246.

[20] Pei, S.; Cheng, H. M. The reduction of graphene oxide. Carbon 2012, 50(9), 3210-3228.

[21] Garcia, A.; Albero, J.; Garcia, H. Multilayer N-doped graphene films as electrodes for $\mathrm{H}_{2}$ evolution. ChemPhotoChem 2017, 1(9), 388-392.

[22] Bessegato, G.; Guaraldo, T.; Zanoni, M. Enhancement of photoelectrocatalysis efficiency by using nanostructured electrodes. Modern Electrochemical Methods in Nano, Surface and Corrosion Science. InTech, 2014. 

Chapter 4

Catalyst-free one step synthesis of large area, vertically stacked $\mathrm{N}$-doped graphene-boron nitride heterostructures from biomass precursors

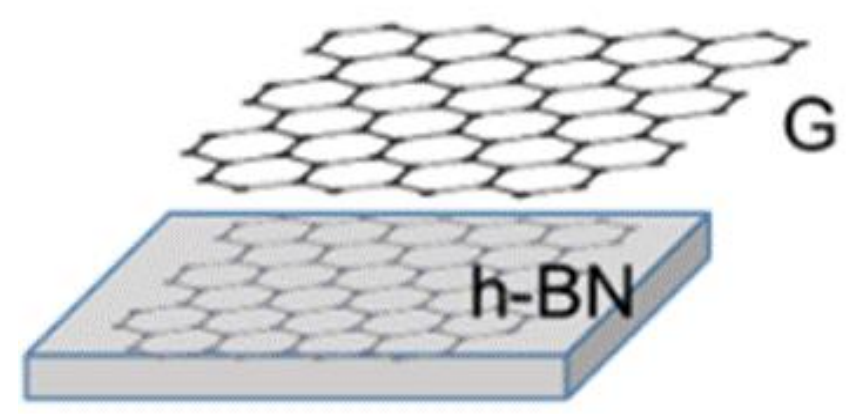





\subsection{Introduction}

Due to the numerous potential applications in various fields from microelectronics ${ }^{[1-3]}$ to optical materials, ${ }^{[4-7]}$ there is much current interest in developing novel procedures for the preparation of large-area films of graphene and related materials. ${ }^{[8-10]}$ As commented in the Introduction, we have been reporting in a series of papers ${ }^{[11-17]}$ that pyrolysis of filmogenic natural polysaccharides, such as chitosan and alginate, at temperatures of $900{ }^{\circ} \mathrm{C}$ or above in the absence of oxygen forms films of defective undoped or doped graphenes. The key features responsible for the formation of defective graphene films are the tendency of polysaccharides to decompose into turbostratic graphitic carbon residues and their ability to form thin nanometric, defectless, continuous, conformal films on arbitrary surfaces with subnanometric rugosity. Upon pyrolysis, these films of filmogenic polysaccharides of about $10 \mathrm{~nm}$ thickness undergo shrinking and transformation into mono- or few layers graphene films. ${ }^{[15]}$ The procedure can be modified using derivatives of these polysaccharides with inorganic acids to form defective graphene films containing dopant elements, including $S, B$ and $P$ as well as co-doping of these elements. ${ }^{[13,14,16,18-22]}$

In previous work from our group, following a similar procedure, the pyrolytic process of polysaccharide films was modified to afford graphene films having oriented MNPs as well as metal oxides and sulfides. ${ }^{[18,23-33]}$ In the last case, the precursors of these films are the filmogenic polysaccharides whose fibrils contain a salt of the transition metal or a polysulfide that undergo chemical reduction under pyrolysis conditions in the absence of oxygen. Spontaneous segregation of two separate phases, graphene and the metal or metal sulfide, can take place in those cases in which the metal does not form the corresponding carbides. Besides films, a similar procedure can be adapted for the preparation of graphitic carbon residues as powders that upon sonication in a liquid media give rise to doped graphenes and graphenes supporting NPs. 
Continuing this research line, the present Chapter reports the one-step preparation of films of defective $\mathrm{N}$-doped graphene on a nanometric layer of boron nitride ((N)graphene/h-BN) on quartz. Characterization data based on optical and electronic microscopy as well as XPS with angular resolution shows that graphene sheet covers the bottom boron nitride layer, the latter exhibiting the typical cracked morphology ${ }^{[34-36]}$ originated as consequence of the vitreous nature of boron nitride and thermal stress suffered by (N)graphene/h-BN during the pyrolysis.

\subsection{Results and discussion}

The process of the formation of films is illustrated in Scheme 4.1. A thin film of chitosan containing ammonium borate was spin cast on a freshly cleaned quartz surface (see Chapter 8 for the experimental representative weights and procedures). The ability of chitosan to adsorb ions from aqueous solution has been exploited in fields like water purification. ${ }^{[37-39]}$ Similarly, in the present Chapter $\left(\mathrm{NH}_{4}\right)_{3} \mathrm{BO}_{3}$ was adsorbed on chitosan. Up to $20 \mathrm{wt} \%$ of $\left(\mathrm{NH}_{4}\right)_{3} \mathrm{BO}_{3}$ could be adsorbed on chitosan.

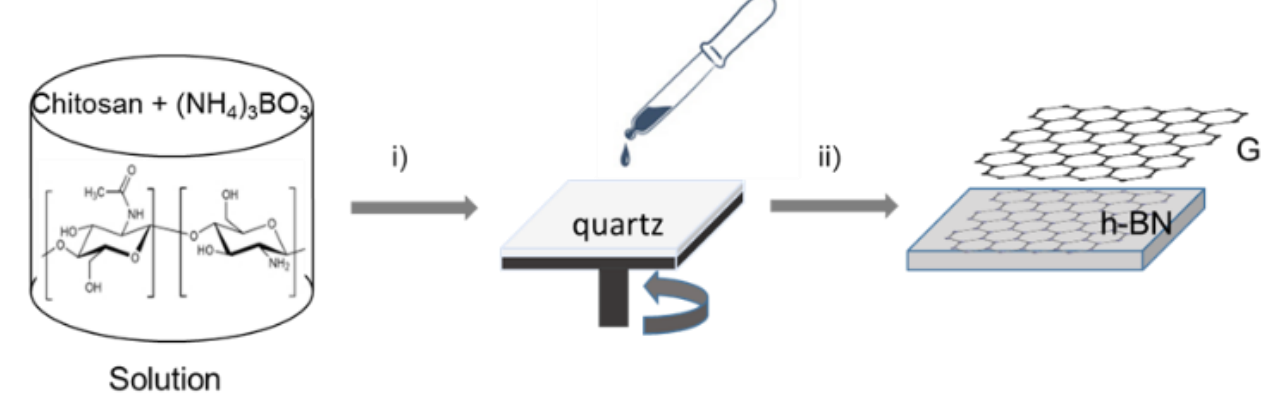

Scheme 4.1. Preparation procedure for (N)graphene/h-BN film on quartz. i) spin coating; ii) pyrolysis at $900{ }^{\circ} \mathrm{C}$ under $\mathrm{Ar}$ atmosphere.

Formation of single-layer boron nitride films has been reported in the pyrolysis of aminoborane. ${ }^{[40-42]}$ However, since aminoborane is volatile and inadequate for our 
pyrolytic process, a salt as ammonium borate was considered as a more suitable precursor to be combined with chitosan. Precedents have allowed the preparation of suspended boron nitride NPs in aqueous phase by pyrolysis of $\left(\mathrm{NH}_{4}\right)_{3} \mathrm{BO}_{3}$ adsorbed on chitosan, followed by combustion of carbonaceous materials and sonication. ${ }^{[43-44]}$ An analogous process resulting in the formation of boron nitride was expected to occur in the present case, but the configuration of the precursor as a film, rather than as powder, could allow the spontaneous segregation of the material during the pyrolysis in two independent phases.

Our hypotheses were confirmed as evidenced by optical microscopy. Figure 4.1 shows optical microscopy images of the $(\mathrm{N})$ graphene/h-BN film resulting upon pyrolysis of chitosan embedding $\left(\mathrm{NH}_{4}\right)_{3} \mathrm{BO}_{3}$ and the corresponding $\mathrm{h}-\mathrm{BN}$ film that can be obtained by subsequent air calcination at $1000{ }^{\circ} \mathrm{C}$ to remove graphene. As it can be seen there, the (N)graphene/h-BN film clearly exhibits two-layers heterostructures, in which a thin film of graphene with the gray appearance is observed on top of a denser layer full of cracks. It is likely that during the pyrolysis and phase segregation the two independent phases with different density become spatially arranged in the way that the less dense phase appears on the top of the densest phase. The interaction between the two independent layers will be mainly van der Waals forces and $\pi-\pi$ interactions. Although graphene films on BN have been prepared by CVD, micromanipulation and other methods, the present procedure has the advantage of using biomass precursors and ammonium borate salt and can be applied to obtain large area films $\left(1 \times 1 \mathrm{~cm}^{2}\right)$ on arbitrary substrates.

Besides, the image of $\mathrm{h}-\mathrm{BN}$ sample resulting after air calcination and removal of the graphene overlayer shows white, smooth and highly cracked films deposited on quartz. In the existing precedents in the literature on the preparation of boron nitride films, ${ }^{[45]}$ similar images of a fully cracked layer have been reported as typical of boron nitride. Notice that while graphene is a highly elastic material, the thermal stress originated in the high-temperature formation of boron nitride with vitreous nature and subsequent cooling at room temperature is responsible for the cracking of the 
boron nitride film. This behavior is in contrast with the high elastic properties of graphene that does not undergo film cracking upon similar thermal treatments. ${ }^{[46-47]}$
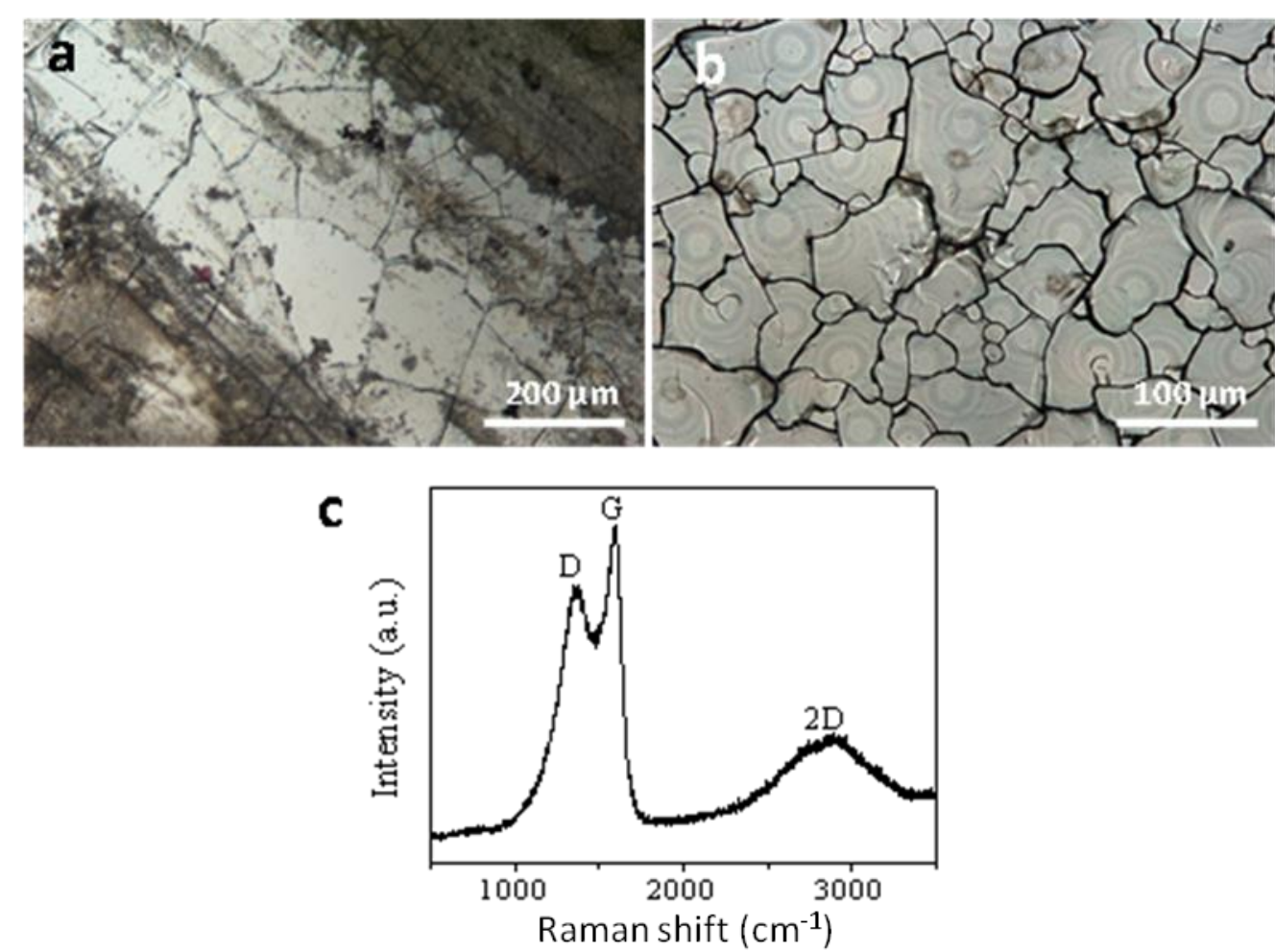

Figure 4.1. Optical microscopy images of the $(N)$ graphene/h-BN film (a) with a handmade scratch at the center and h-BN film (b) on quartz after combustion of graphene. Notice in the image a that the gray tint at the upper right and lower left corners is due to the presence of graphene and in those parts in which the graphene layer has been detached by the scratch, the color of the boron nitride layer underneath is white. Frame c shows the Raman spectra of $(\mathrm{N})$ graphene/h-BN obtained upon excitation at $514 \mathrm{~nm}$.

Convincing evidence of the formation of $(\mathrm{N})$ graphene was obtained by Raman spectroscopy of the films. While boron nitride has a very weak Raman signal, it is known ${ }^{[48-50]}$ that Raman spectroscopy is a highly sensitive technique to detect and characterize even single-layer graphene. In the present case, Raman spectra of the (N)graphene film on boron nitride layer show the characteristic 2D, G and D peaks appearing at 2900, 1580 and $1354 \mathrm{~cm}^{-1}$, respectively, expected for defective 
graphenes derived from chitosan (Figure 4.1a, inset). Observation of those signals in Raman spectroscopy is a strong evidence that graphene films have been formed in agreement with the known behavior of chitosan.

The thickness and roughness of the graphene and boron nitride layers were determined by AFM measurements. To check the layered structure of the stacked (N)graphene/h-BN film, a scratch on the as-synthesized film was made with a sharp blade. The representative AFM image of a relatively large area $\left(1 \times 1 \mu m^{2}\right)$ centered on the scratch is shown in Figure 4.2a. The flat film monitored by AFM is in accordance with the existence of a smooth upper graphene film on top of the cracked boron nitride layer. The section profile throughout the films to the quartz substrate is shown in Figure 4.2c, from which the thicknesses of graphene and boron nitride layer can be easily determined as about 3 and $2 \mathrm{~nm}$, respectively, corresponding to few-layers graphene and h-BN. Also, a frontal AFM image of the h-BN film after removal of graphene by combustion shows a uniform surface with the cracks and crevices that are observed in optical microscopy. 

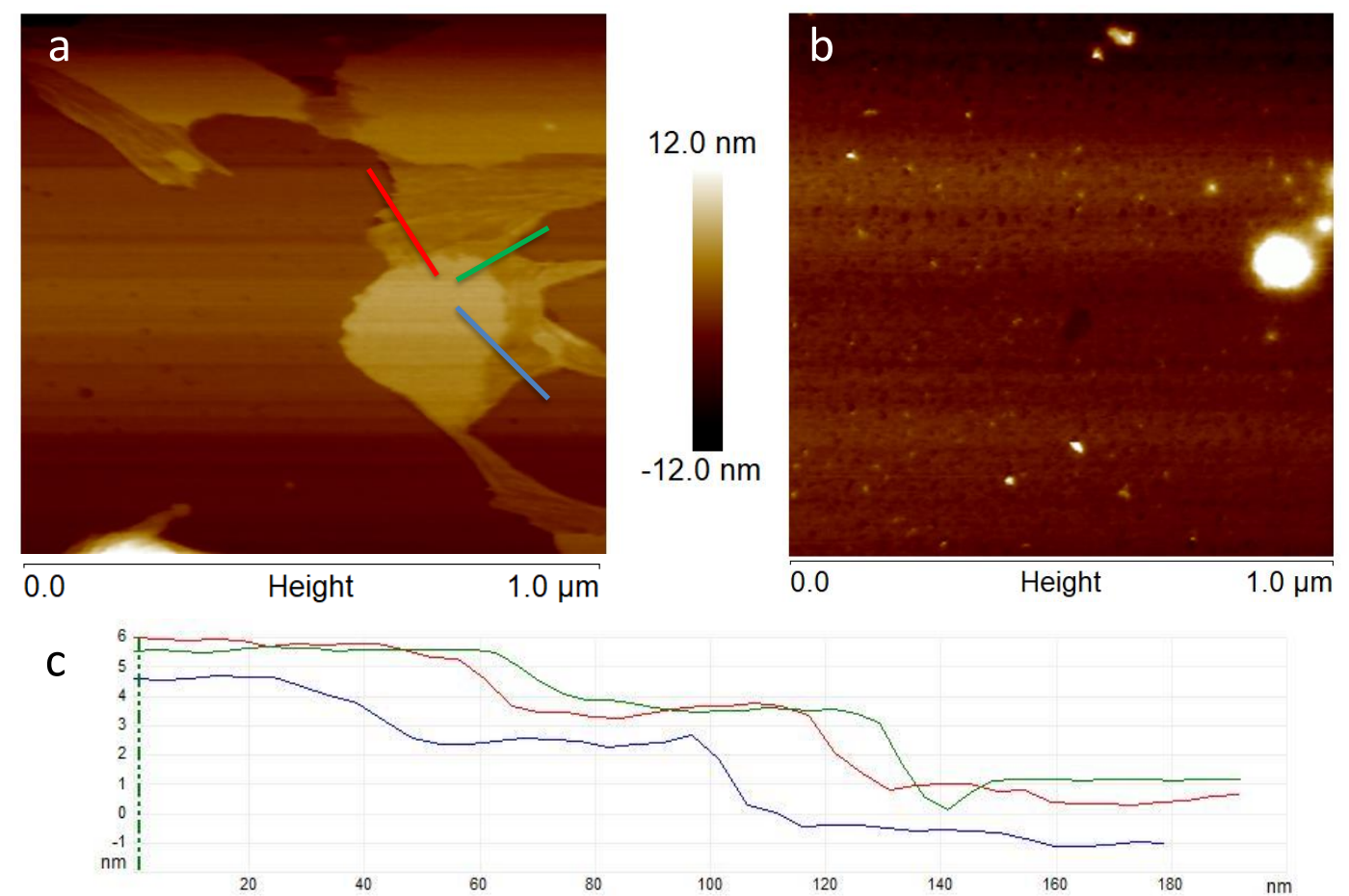

Figure 4.2. Frontal AFM images around a scratch of the graphene/h-BN film (a) showing the different phases of (N)graphene, h-BN, and quartz and (b) a frontal view of the h-BN film after graphene combustion showing the morphology of the surface. Frame c presents the measurement of the thickness of $(\mathrm{N})$ graphene/h-BN film along the blue, red and green lines indicated with the same colors in image a.

Scanning electron microscopy (SEM) can also provide useful information about the $(\mathrm{N})$ graphene/h-BN heterostructures. To get better images of $(\mathrm{N})$ graphene/h-BN films, the surface of the film was slightly damaged with a spatula. As show in Figure 4.3, this debris detached from the quartz substrate scratch of h-BN exhibits a characteristic morphology of thin platelets having straight periphery in accordance with the images by optical microscopy attributed to h-BN. 

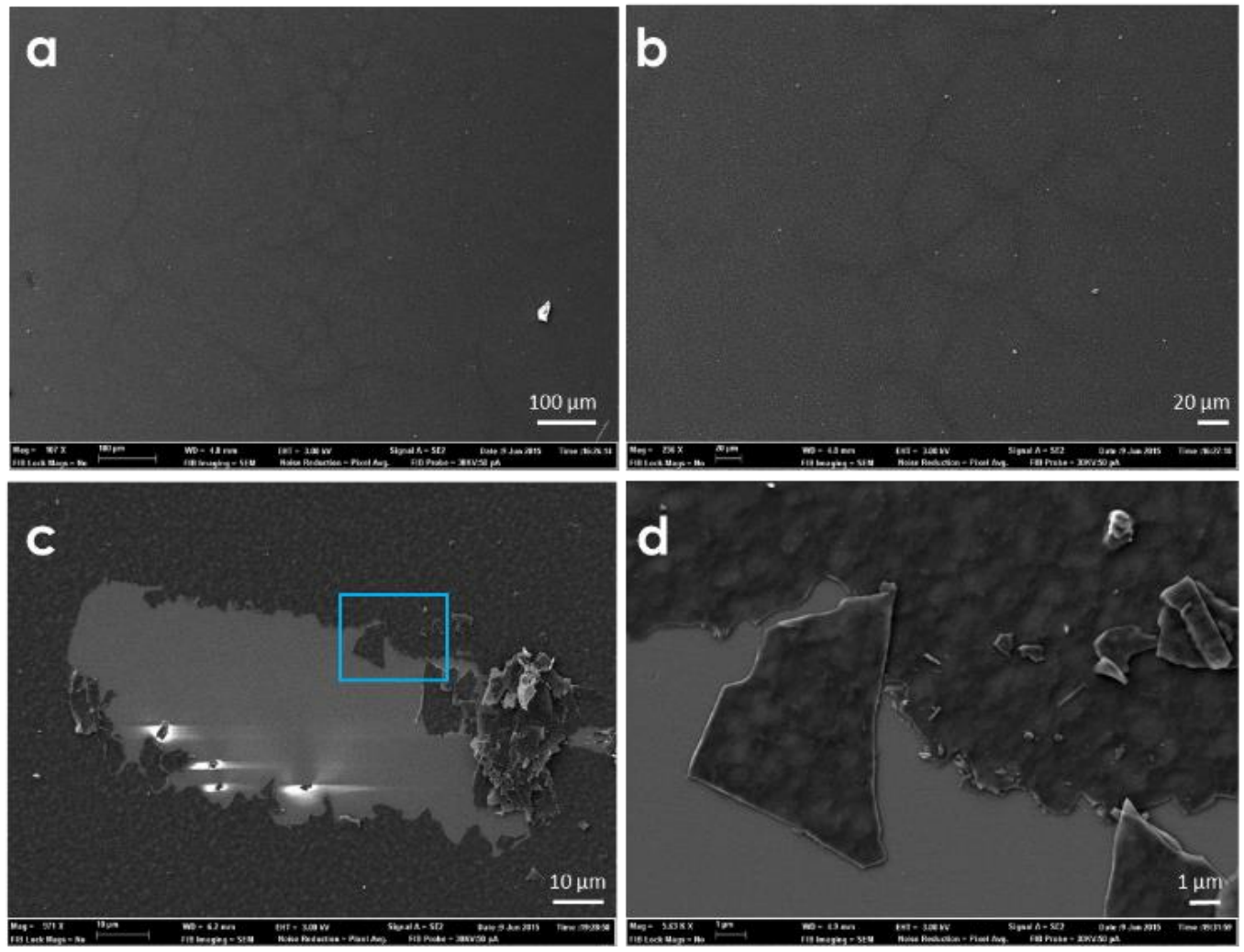

Figure 4.3. (a and b) SEM images of (N)graphene/h-BN heterostructures on quartz substrate at two different magnifications. (c) Images of the region of the graphene/h-BN film damaged with a spatula. (d) Image at higher magnification of the rectangular region marked in blue in image $\mathbf{c}$ showing well detached $\mathrm{h}$-BN platelets.

SEM images were complemented by cross-sectional views obtained applying fast ion bombardment (FIB) technique. The FIB treatment uses a finely focused beam of high energetic gallium ions operating at high currents that produce a sharp cut in the material allowing to obtain an image of the film profile. The resulting cross-section image obtained by FIB-SEM for a special (N)graphene/h-BN film is shown in Figure 4.4. Notice that in order to obtain a clearer cross-section image, the graphene/h-BN film submitted to FIB was somewhat thicker than the one corresponding to the AFM measurements shown in Figure 4.2 that was much thinner. Two layers of different materials can be observed in this cross-sectional image. 

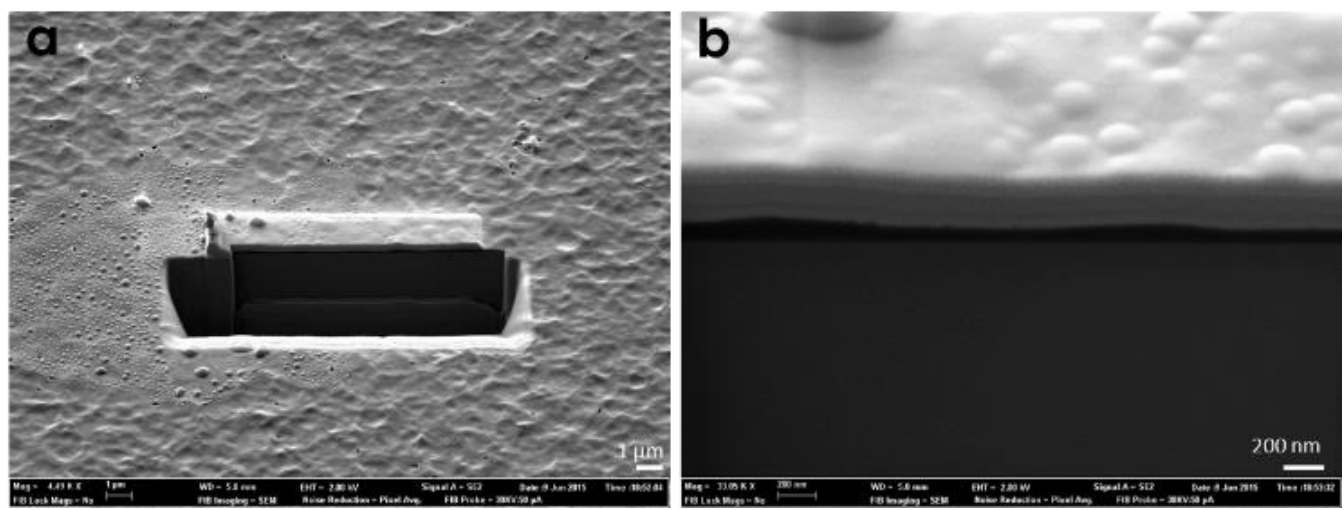

Figure 4.4. a) SEM image of a (N)graphene/h-BN film that has been submitted to FIB. b) Magnification of the cross-section obtained by FIB in which two layers corresponding to graphene and $\mathrm{h}-\mathrm{BN}$ can be observed from the somewhat different contrast.

By scratching small debris of the heterostructure present on the film onto the grid suitable as sample holder for TEM, high-resolution transition electron microscopy (HRTEM) images of the heterostructure could be taken. Figure 4.5 shows representative images of the morphology of $(\mathrm{N})$ graphene/h-BN sample detached from the film. It should be remarked that the sheet structures of graphene and h-BN are very similar, which makes it difficult to distinguish them by this technique.
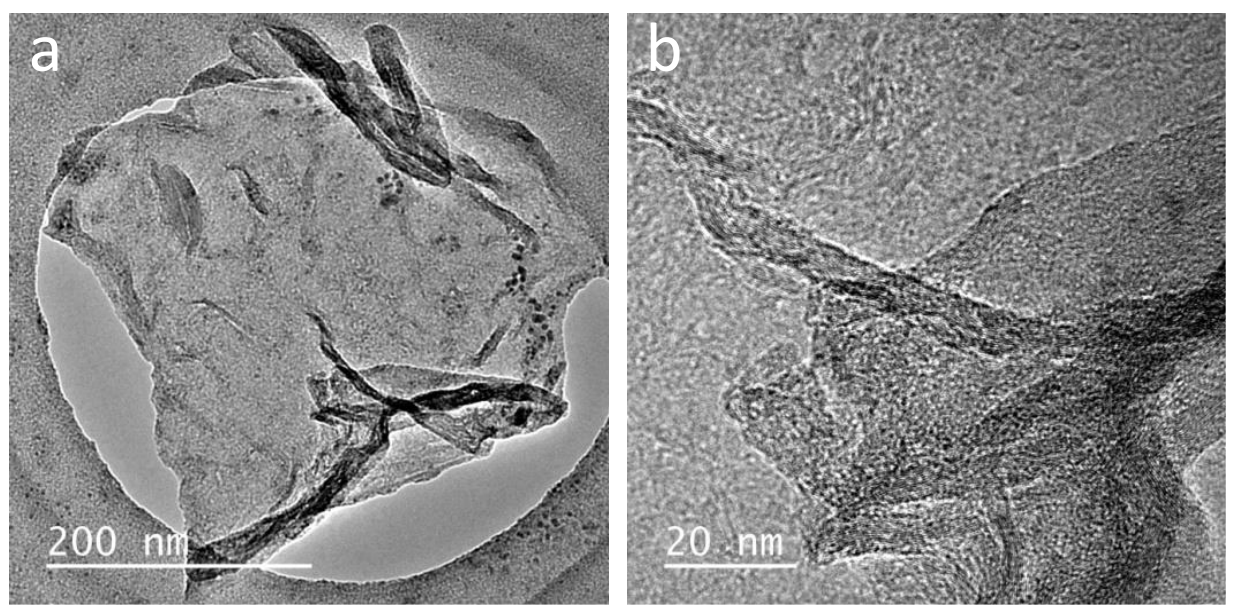

Figure 4.5. HRTEM images of the (N)graphene/h-BN heterostructures detached from the films showing the morphology of the sheet structure at different magnifications. 
Further evidence that $(\mathrm{N})$ graphene film is overlaying boron nitride could be obtained by XPS analysis measuring the spectra at different monitoring angles. Thus, in accordance with the film morphology, X-ray excitation perpendicular to the film at $0^{\circ}$ monitoring angle revealed the presence of $\mathrm{C}, \mathrm{B}$ and $\mathrm{N}$ in an approximately relative atomic proportion of 16.5:1:1. Analysis of the high resolution XPS peaks corresponding to the $\mathrm{C} 1 \mathrm{~s}, \mathrm{~B} 1 \mathrm{~s}$ and N1s peaks were in agreement with the reported values for defective graphene derived from chitosan and for boron nitride, respectively. Figure 4.6(a-c) presents the experimental XPS peaks for these elements and their best deconvolution to individual components. As it can be seen there, besides graphenic $\mathrm{C}$ in $68 \%$, the presence of $\mathrm{C}$ atoms bonded to $\mathrm{N}$ or $\mathrm{O}$ and carboxylic acid groups with binding energy values of 285.9 and $288.4 \mathrm{eV}$ were also observed.

On the contrary, the XPS B1s peak deconvolution indicates that this peak corresponds to a single component at a binding energy of $190.7 \mathrm{eV}$ that agrees with the expected value for $B$ atoms in boron nitride. ${ }^{[51-53]}$ This binding energy and the symmetry of the B1s peak rules out the formation of B-doped graphene in a measurable extent. In the case of N1s, the XPS peak can also be fit to one single component with a binding energy value of $398.3 \mathrm{eV}$ that also agrees with the expected value for $\mathrm{N}$ atoms of boron nitride. Additional contribution of $\mathrm{N}$ atoms on $\mathrm{N}$-doped graphene were very minor compared to that of boron nitride and could not be determined adequately from the experimental N1s peak. 

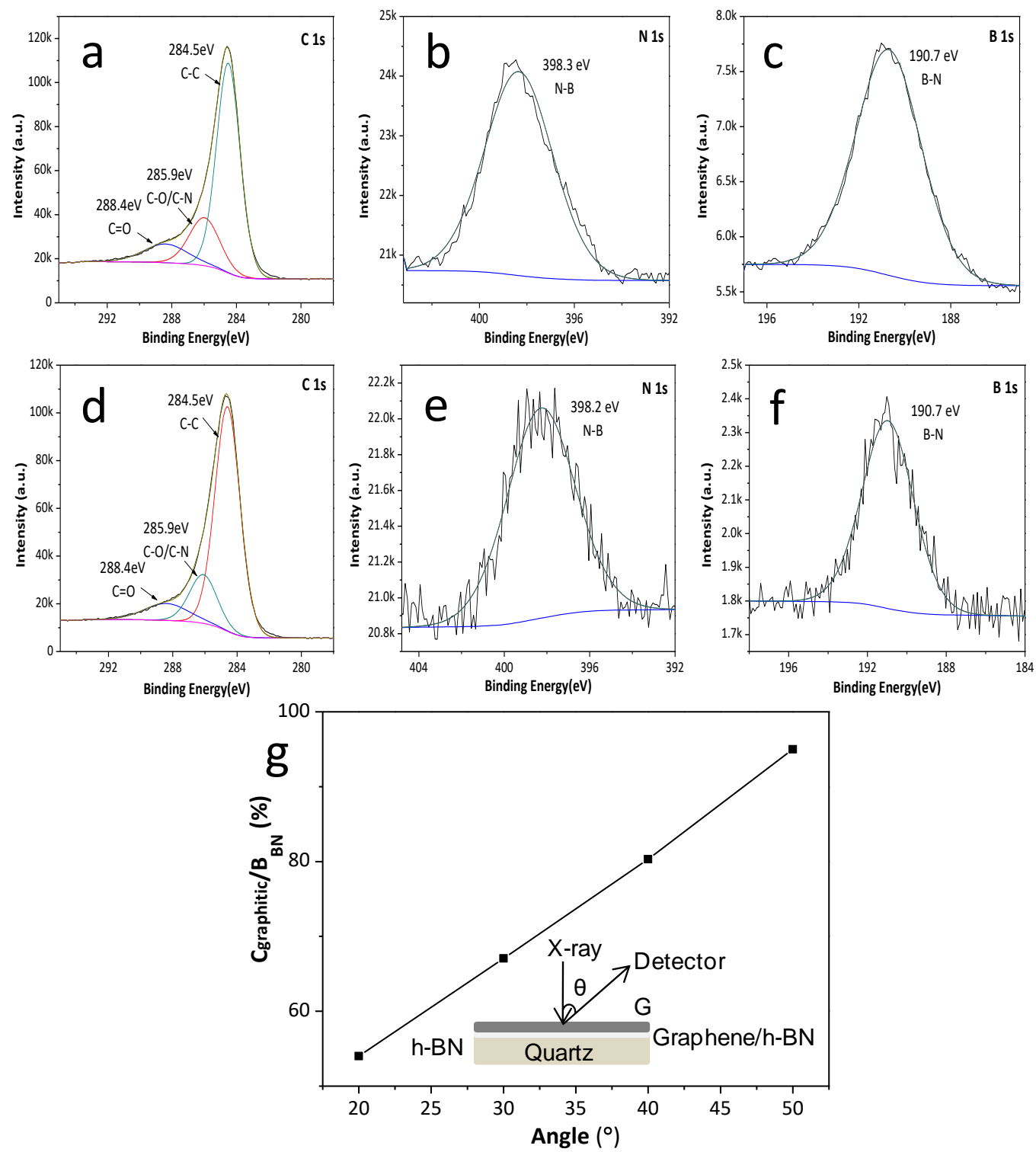

Figure 4.6. High-resolution XPS peaks of C1s, N1s, B1s obtained by analysis at a monitoring angle of $0^{\circ}(\mathrm{a}-\mathrm{c})$ and $50^{\circ}$ (d-f) for (N)graphene/h-BN film, respectively. (g) Plot of the graphitic carbon vs B atomic ratio as a function of the detector angle. The inset presents the relative geometry of exciting X-ray and detection angle $(\theta)$ vs the surface of the (N)graphene/h-BN film.

By changing the monitoring angle of the ejected electrons in XPS, the atomic proportion of $\mathrm{B}$ and $\mathrm{N}$ vs. $\mathrm{C}$ atoms decreased simultaneously in identical magnitude. A minimal relative atomic value of approximately 10.5:0.1:0.1 for C, B and $\mathrm{N}$ 
elements at a monitoring angle of $50^{\circ}$ was obtained. Figure $4.6(\mathrm{~d}-\mathrm{f})$ presents the XPS peaks of these elements obtained with a $\theta$ tilt angle of $50^{\circ}$ as well as the best deconvolution to the individual components. These XPS measurements at different tilt angles are in agreement with the images of optical microscopy showing that graphene forms an upper layer over boron nitride in (N)graphene/h-BN. Thus, when analysis is perpendicular to the surface, all the elements in the corresponding proportion were detectable by XPS, while moving the detector in the direction parallel to the surface, an increase in the proportion of graphene over that of boron nitride was observed. Figure $4.6 \mathrm{~g}$ presents a plot of the graphitic C vs B atomic ratio as a function of the detector $\theta$ tilt angle from the surface perpendicular in which a linear relationship can be observed.

It is well-known that electrical conductivity of graphenes depends on many different chemical and experimental factors, including the substrate on which it is supported. The record of maximal electron mobility for graphenes has been reported for defectless graphene deposited on boron nitride forming an aligned superlattice. ${ }^{[54-58]}$ It seems that the excellent matching between the lattices of two 2D materials and their alignment weak interaction through van der Waals forces are among the main reason for this high electron mobility. In the present (N)graphene/h-BN material, it is clear that the defective nature of graphene prepared from pyrolysis of chitosan containing $\mathrm{N}$-dopant atoms, as well as residual oxygenated functional groups and carbon vacancies decreases considerably its electrical conductivity compared to that of ideal graphene. However, this type of measurements of electrical conductivity are of large importance to understand the properties of this $\mathrm{N}$-doped graphene on top of boron nitride, particularly compared to the behavior of the same type of defective $\mathrm{N}$-doped graphene on quartz substrate. The maximal electrical conductivity measured by the four tips head was $10 \mathrm{k} \Omega / \mathrm{sq}$ (sq, meaning square) that is even lower than the value that has been measured for $\mathrm{N}$-doped graphene on quartz $(2 \mathrm{k} \Omega / \mathrm{sq})$. Thus, it seems that the presence of cracks on the $\mathrm{h}-\mathrm{BN}$ layer and/or the interaction between the $(\mathrm{N})$ graphene and $\mathrm{h}-\mathrm{BN}$ layers do 
not allow an improvement of the surface electrical conductivity at the millimetric length scale. Graphene and BN layers can interact in various ways and when the alignment of the hexagons of both materials match perfectly a superlattice structure may appear. ${ }^{[59]}$ The presence of cracks in $\mathrm{BN}$ and $\mathrm{N}$-doping in graphene, the various ways of interaction between these two layers and the match or disorder between the two layers should be responsible for the differences in the electrical conductivity measured for the assembly compared to the independent $\mathrm{N}$-doped graphene.

To gain further insight on the electrical behavior of $(\mathrm{N})$-graphene/h-BN films, additional electrical measurements using a micrometric tip electrode were carried out (see schematic of the setup in Figure 4.7a). The purpose was to determine the electrical conductivity at dimensions commensurate with the h-BN islands, several of which have a submillimetric dimensions, according to optical microscopy. When performing the initial screening with $\mathrm{mA}$ currents, complete combustion of the (N)graphene layer was observed. This indicates that the internal resistance of the (N)graphene causes heating of the material, reaching the high temperatures needed to cause combustion of the material.

Independent thermogravimetric analysis of the $(\mathrm{N})$ graphene/h-BN film shows an initial weight loss of about $4 \%$ at temperatures below $200{ }^{\circ} \mathrm{C}$ corresponding to the desorption of water and other adsorbates, followed by a $14 \%$ weight loss in the range of temperatures between 600 and $800{ }^{\circ} \mathrm{C}$ that is due to the combustion of the $(\mathrm{N})$ graphene layer. Figure $4.7 \mathrm{~b}$ presents the weight loss profile as a function of the temperature corresponding to the thermogravimetric analysis of $(\mathrm{N})$ graphene/h-BN film. Therefore, it seems that these high electrical currents should heat the (N)graphene layer enough to reach locally the high temperatures needed to trigger the combustion of the graphene materials that is estimated about $600{ }^{\circ} \mathrm{C}$. Lowering the current intensity to $\mathrm{nA}$ values allowed to confirm a poor electrical conductivity for the (N)graphene/h-BN films in the range of a few $\mathrm{k} \Omega / \mathrm{sq}$, even at these submicrometric distances, measuring on various positions of the $1 \times 1 \mathrm{~cm}^{2}$ area of the film, with relatively minor dispersion on the values. However, these electrical 
measurements allowed to monitor a capacitative behavior of the $(\mathrm{N})$ graphene/h-BN heterostructure in the positive potential region. Figure $4.7 \mathrm{~d}$ shows the current intensity vs. applied voltage from which resistance was determined and how at positive voltages a hysteresis loop is observed. The I-V plot shown in Figure $4.7 \mathrm{~d}$ was reproducible in several cycles at the same position of the electrode or in different parts of the $(\mathrm{N})$ graphene/h-BN film, indicating that the process of charge and discharge at these current densities, voltages and scan rates is reversible and does not cause any apparent damage to the film.

a)

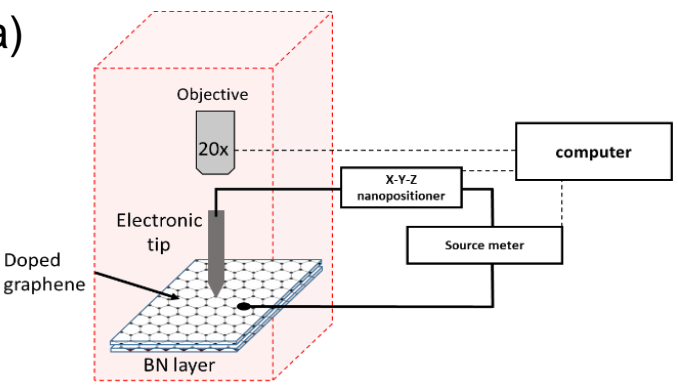

c)

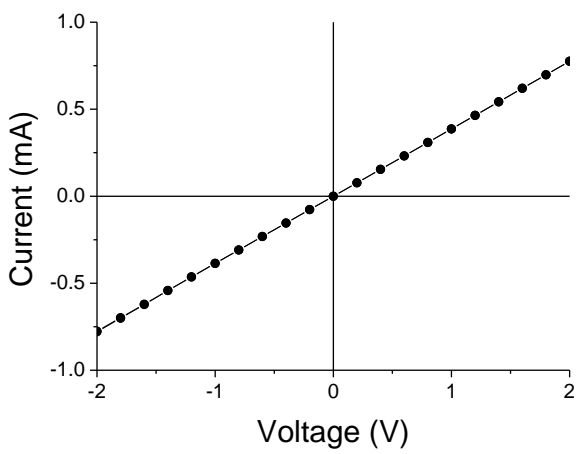

b)

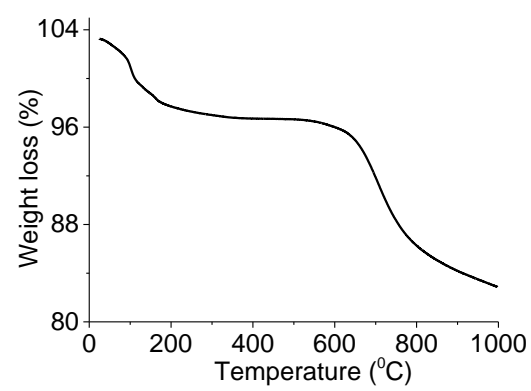

d)

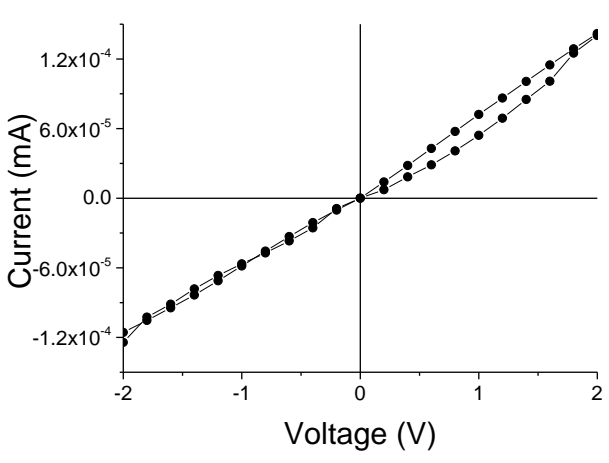

Figure 4.7. a) Schematic representation of the setup used for measuring the current-voltage curves at submillimetric scale. b) TG measurement of the (N)graphene/h-BN. c) I-V curves measured for (N)graphene on quartz and (d) (N)graphene/h-BN. The $\mathrm{I}-\mathrm{V}$ curves were measured at a scan rate of $200 \mathrm{mV} / \mathrm{s}$ between -2 to $2 \mathrm{~V}$.

The most reasonable explanation for the hysteresis is that the (N)graphene/h-BN heterostructure exhibits a capacitative behavior storing charge upon scanning at positive potentials. The specific accumulated charge as Faraday 
divided per gram of material could be determined from the I-V plots presented in Figure $4.7 \mathrm{~d}$, giving a value of $117{\mathrm{~F} \times g^{-1}}^{-1}$ that is in line with the charge accumulation data reported for single layer BN prepared by exfoliation of commercial bulk $\mathrm{h}-\mathrm{BN}$. ${ }^{[60]}$ This indicates that our ( $\mathrm{N}$ )graphene/h-BN film can act as a capacitor storing charge and this film can be used in microchips and devices with micrometric dimensions as reversible charge accumulation system.

\subsection{Conclusions}

The present Chapter has shown the one-step procedure for the preparation of defective $\mathrm{N}$-doped graphene on top of a boron nitride layer that can be applied for the preparation of large $\left(\mathrm{cm}^{2}\right)$ areas of this composite film on arbitrary substrates. Formation of the film derives from the spontaneous segregation of independent layers of graphene and boron nitride as the chitosan precursor embedding a high percentage of ammonium borate is submitted to pyrolysis. The procedure disclosed in the present Chapter illustrates the adaptability of defective graphene synthesis based on the use of filmogenic natural polysaccharides in the preparation of various graphene heterojunctions. The film exhibits high electrical resistance in the range of $10 \mathrm{k} \Omega / \mathrm{sq}$ with no improvement in electrical conductivity with respect to the conductivity values of $(\mathrm{N})$ graphene. This high electrical resistance is responsible for the combustion of the $(\mathrm{N})$ graphene layer when the film is submitted to currents in the $\mathrm{mA}$ range. In contrast, the film can be submitted to nA currents without causing its combustion. In this current range, the $(\mathrm{N})$ graphene/h-BN film exhibits a reversible and stable capacitative behavior, with a charge accumulation estimated in $117{\mathrm{~F} \times \mathrm{g}^{-1}}^{-}$. The above results represent a step forward towards the preparation of graphene-boron nitride superlattices in a single pyrolytic process of a precursor mainly constituted by biomass waste. 


\subsection{References}

[1] Frazier, R. M.; Daly, D. T.; Swatloski, R. P.; Hathcock, K. W.; South, C. R. Recent progress in graphene-related nanotechnologies. Recent Patents on Nanotechnology 2009, 3(3), 164-176.

[2] Mattevi, C.; Colleaux, F.; Kim, H. K.; Lin, Y. H.; Park, K. T.; Chhowalla, M.; Anthopoulos, T. D. Solution-processable organic dielectrics for graphene electronics Nanotechnology 2012, 23(34), 344017-344026.

[3] Vaziri, S.; Lupina, G.; Paussa, A.; Smith, A. D.; Henkel, C.; Lippert, G.; Dabrowski, J.; Mehr, W.; Oestling, M.; Lemme, M. C. A graphene-based hot electron transistor. Nano Letters 2013, 13(4), 1435-1439.

[4] Constant, T. J.; Hornett, S. M.; Chang, D. E.; Hendry, E. All-optical generation of surface plasmons in graphene. Nature Physics 2016, 12(2), 124-127.

[5] Gu, M.; Zhang, Q.; Lamon, S. Nanomaterials for optical data storage. Nature Reviews Materials 2016, 1(12), 16070.

[6] He, Z.; Zhong, C.; Huang, X.; Wong, W. Y.; Wu, H.; Chen, L.; Cao, Y. Simultaneous enhancement of open-circuit voltage, short-circuit current density, and fill factor in polymer solar cells. Advanced Materials 2011, 23(40), 4636-4643.

[7] Yu, X.; Cheng, H.; Zhang, M.; Zhao, Y.; Qu, L.; Shi, G. Graphene-based smart materials. Nature Reviews Materials 2017, 2(9), 17046.

[8] Guo, S.; Dong, S. Graphene nanosheet: synthesis, molecular engineering, thin film, hybrids, and energy and analytical applications. Chemical Society Reviews 2011, 40(5), 2644-2672.

[9] Tan, L.; Zeng, M.; Zhang, T.; Fu, L. Design of catalytic substrates for uniform graphene films: from solid-metal to liquid-metal. Nanoscale 2015, 7(20), 9105-9121.

[10] Xu, M.; Liang, T.; Shi, M.; Chen, H. Graphene-like two-dimensional materials. Chemical Reviews 2013, 113(5), 3766-3798.

[11] Atienzar, P.; Primo, A.; Lavorato, C.; Molinari, R.; Garcia, H. Preparation of graphene quantum dots from pyrolyzed alginate. Langmuir 2013, 29(20), 6141-6146. [12] Primo, A.; Forneli, A.; Corma, A.; Garcia, H. From biomass wastes to highly 
efficient $\mathrm{CO}_{2}$ adsorbents: graphitisation of chitosan and alginate biopolymers. ChemSusChem 2012, 5(11), 2207-2214.

[13] Dhakshinamoorthy, A.; Primo, A.; Concepcion, P.; Alvaro, M.; Garcia, H. Doped graphene as a metal-free carbocatalyst for the selective aerobic oxidation of benzylic hydrocarbons, cyclooctane and styrene. Chemistry - A European Journal 2013, 19(23), 7547-7554.

[14] Latorre-Sanchez, M.; Primo, A.; Garcia, H. P-doped graphene obtained by pyrolysis of modified alginate as a photocatalyst for hydrogen generation from water-methanol mixtures. Angewandte Chemie - International Edition 2013, 52(45), 11813-11816.

[15] Primo, A.; Atienzar, P.; Sanchez, E.; Delgado, J. M.; Garcia, H. From biomass wastes to large-area, high-quality, N-doped graphene: catalyst-free carbonization of chitosan coatings on arbitrary substrates. Chemical Communications 2012, 48(74), 9254-9256.

[16] Primo, A.; Sanchez, E.; Delgado, J. M.; Garcia, H. High-yield production of $\mathrm{N}$-doped graphitic platelets by aqueous exfoliation of pyrolyzed chitosan. Carbon $2014,68,777-783$.

[17] Dhakshinamoorthy, A.; Primo, A.; Concepcion, P.; Alvaro, M.; Garcia, H. Doped graphene as a metal-free carbocatalyst for the selective aerobic oxidation of benzylic hydrocarbons, cyclooctane and styrene. Chemistry - A European Journal 2013, 19(23), 7547-7554.

[18] Blez, J. F.; Primo, A.; Asiri, A. M.; Alvaro, M.; Garcia, H. Copper nanoparticles supported on doped graphenes as catalyst for the dehydrogenative coupling of silanes and alcohols. Angewandte Chemie - International Edition 2014, 53(46), $12581-12586$.

[19] Dhakshinamoorthy, A.; Latorre-Sanchez, M.; Asiri, A. M.; Primo, A.; Garcia, H. Sulphur-doped graphene as metal-free carbocatalysts for the solventless aerobic oxidation of styrenes. Catalysis Communications 2015, 65, 10-13.

[20] Esteve-Adell, I.; Crapart, B.; Primo, A.; Serp, P.; Garcia, H. Aqueous phase reforming of glycerol using doped graphenes as metal-free catalysts. Green 
Chemistry 2017, 19(13), 3061-3068.

[21] Latorre-Sanchez, M.; Primo, A.; Atienzar, P.; Forneli, A.; Garcia, H. P-n heterojunction of doped graphene films obtained by pyrolysis of biomass precursors. Small 2015, 11(8), 970-975.

[22] Lavorato, C.; Primo, A.; Molinari, R.; Garcia, H. N-doped graphene derived from biomass as a visible-light photocatalyst for hydrogen generation from water/methanol mixtures. Chemistry - A European Journal 2014, 20(1), 187-194.

[23] Blandez, J. F.; Esteve-Adell, I.; Primo, A.; Alvaro, M.; Garcia, H. Nickel nanoparticles supported on graphene as catalysts for aldehyde hydrosilylation. Journal of Molecular Catalysis A: Chemical 2016, 412, 13-19.

[24] Dhakshinamoorthy, A.; Esteve Adell, I.; Primo, A.; Garcia, H. Enhanced activity of $\mathrm{Ag}$ nanoplatelets on few layers of graphene film with preferential orientation for dehydrogenative silane-alcohol coupling. ACS Sustainable Chemistry and Engineering 2017, 5(3), 2400-2406.

[25] Esteve-Adell, I.; Bakker, N.; Primo, A.; Hensen, E.; Garcia, H. Oriented Pt nanoparticles supported on few-layers graphene as highly active catalyst for aqueous-phase reforming of ethylene glycol. ACS Applied Materials and Interfaces 2016, 8(49), 33690-33696.

[26] Frindy, S.; El Kadib, A.; Lahcini, M.; Primo, A.; Garcia, H. Copper nanoparticles stabilized in a porous chitosan aerogel as a heterogeneous catalyst for C-S cross-coupling. ChemCatChem 2015, 7(20), 3307-3315.

[27] Frindy, S.; El Kadib, A.; Lahcini, M.; Primo, A.; Garcia, H. Nanosized copper supported on graphene as catalyst for the oxidative C-O cross-coupling of phenols. Chemistry Select 2016, 1(2), 157-162.

[28] Frindy, S.; El Kadib, A.; Lahcini, M.; Primo, A.; Garcia, H. Isotropic and oriented copper nanoparticles supported on graphene as aniline guanylation catalysts. ACS Catalysis 2016, 6(6), 3863-3869.

[29] Frindy, S.; El Kadib, A.; Lahcini, M.; Primo, A.; Garcia, H. Copper nanoparticles supported on graphene as an efficient catalyst for $A_{3}$ coupling of benzaldehydes. Catalysis Science \& Technology 2016, 6(12), 4306-4317. 
[30] He, J.; Dhakshinamoorthy, A.; Primo, A.; Garcia, H. Iron nanoparticles embedded in graphitic carbon matrix as heterogeneous catalysts for the oxidative C-N coupling of aromatic N-H compounds and amides. ChemCatChem 2017, 9(15), 3003-3012.

[31] Latorre-Sanchez, M.; Esteve-Adell, I.; Primo, A.; Garcia, H. Innovative preparation of $\mathrm{MoS}_{2}$-graphene heterostructures based on alginate containing $\left(\mathrm{NH}_{4}\right)_{2} \mathrm{MoS}_{4}$ and their photocatalytic activity for $\mathrm{H}_{2}$ generation. Carbon 2015, 81(1), 587-596.

[32] Mateo, D.; Esteve-Adell, I.; Albero, J.; Primo, A.; Garcia, H. Oriented 2.0.0 Cu $2 \mathrm{O}$ nanoplatelets supported on few-layers graphene as efficient visible light photocatalyst for overall water splitting. Applied Catalysis B: Environmental 2017, $201,582-590$.

[33] Primo, A.; Esteve-Adell, I.; Coman, S. N.; Candu, N.; Parvulescu, V. I.; Garcia, H. One-step pyrolysis preparation of 1.1 .1 oriented gold nanoplatelets supported on graphene and six orders of magnitude enhancement of the resulting catalytic activity. Angewandte Chemie - International Edition 2016, 55(2), 607-612.

[34] Mortazavi, B.; Pereira, L. F. C.; Jiang, J. W.; Rabczuk, T. Modelling heat conduction in polycrystalline hexagonal boron-nitride films. Scientific Reports 2015, $5,1-11$

[35] Guo, N.; Wei, J.; Jia, Y.; Sun, H.; Wang, Y.; Zhao, K.; Wu, D. Fabrication of large area hexagonal boron nitride thin films for bendable capacitors. Nano Research 2013, 6(8), 602-610.

[36] Bresnehan, M. S.; Bhimanapati, G. R.; Wang, K.; Snyder, D. W.; Robinson, J. A. Impact of copper overpressure on the synthesis of hexagonal boron nitride atomic layers. ACS Applied Materials and Interfaces 2014, 6(19), 16755-16762.

[37] Kim, H. B.; Kim, J. S.; Hyun, S. Y. Water purification filter using chitosan water purification module, and composite filter system comprising thereof. KR2017006222A 2017.

[38] Theivendran, S.; Hein, S.; Pettibone, M.; Kubinec, J. J. Methods of improving chitosan for water purification in a halogen water treatment system. 
US20130200008A1 2013.

[39] Theivendran, S.; Kubinec, J. J.; Snelling, J. Filter comprising a halogen release system and chitosan for water purification. US20110226706A1 2011.

[40] Behura, S.; Nguyen, P.; Che, S.; Debbarma, R.; Berry, V. Large-area, transfer-free, oxide-assisted synthesis of hexagonal boron nitride films and their heterostructures with $\mathrm{MoS}_{2}$ and $\mathrm{WS}_{2}$. Journal of the American Chemical Society 2015, 137(40), 13060-13065.

[41] Maya, L. Boron nitride precursors: a perspective. Applied Organometallic Chemistry 1996, 10(3-4), 175-182.

[42] Chang, R. J.; Wang, X.; Wang, S.; Sheng, Y.; Porter, B.; Bhaskaran, H.; Warner, J. H. Growth of large single-crystalline monolayer hexagonal boron nitride by oxide-assisted chemical vapor deposition. Chemistry of Materials 2017, 29(15), 6252-6260.

[43] Dhakshinamoorthy, A.; Primo, A.; Esteve-Adell, I.; Alvaro, M.; Garcia, H. Boron nitride nanoplatelets as a solid radical initiator for the aerobic oxidation of thiophenol to diphenyldisulfide. ChemCatChem 2015, 7(5), 776-780.

[44] Primo, A.; Navalon, S.; Asiri, A. M.; Garcia, H. Chitosan-templated synthesis of few-layers boron nitride and its unforeseen activity as a Fenton catalyst. Chemistry A European Journal 2015, 21(1), 324-330.

[45] Shi, Y.; Hamsen, C.; Jia, X.; Kim, K. K.; Reina, A.; Hofmann, M.; Kong, J. Synthesis of few-layer hexagonal boron nitride thin film by chemical vapor deposition. Nano Letters 2010, 10(10), 4134-4139.

[46] Le, M. Q.; Nguyen, D. T. Determination of elastic properties of hexagonal sheets by atomistic finite element method. Journal of Computational and Theoretical Nanoscience 2015, 12(4), 566-574.

[47] Liu, K.; Wu, J. Mechanical properties of two-dimensional materials and heterostructures. Journal of Materials Research 2016, 31(7), 832-844.

[48] Jiang, J.; Pachter, R.; Islam, A. E.; Maruyama, B.; Boeckl, J. J. Defect-induced Raman spectroscopy in single-layer graphene with boron and nitrogen substitutional defects by theoretical investigation. Chemical Physics Letters 2016, 663, 79-83. 
[49] Kim, A.; Lee, M.; Han, S.; Kang, S. J.; Song, K. Raman spectroscopic studies of doped graphene. Polymer (Korea) 2015, 39(6), 956-960.

[50] Koivistoinen, J.; Myllyperkiö, P.; Pettersson, M. Time-resolved coherent anti-stokes Raman scattering of graphene: dephasing dynamics of optical phonon. The Journal of Physical Chemistry Letters 2017, 8(17), 4108-4112.

[51] Ingo, G. M.; Brown, A.; Cossu, G.; Mattogno, G.; Scoppio, L. Second. Ion Mass Spectrom., SIMS 8, Proc. Int. Conf., 8th, 1991, (Pub. 1992), 605.

[52] Millon, E.; Lobstein, N.; Muller, J. F.; Alnot, M. Analyse par spectrometrie de masse et spectroscopie de photoelectrons $X$ de films de nitrure de bore (BN) sur substrat en silicium. Analusis 1995, 1(23), 35-40.

[53] Nemoshkalenko, V. V.; Bochko, A. V.; Senkevich, A. I. Dopov. Akad. Nauk Ukr. RSR, Ser. A: Fiz.-Mat. Tekh. Nauki 1986, 76-78.

[54] Da Silva, A. M.; Jung, J.; Adam, S.; MacDonald, A. H. Fractional hofstadter states in graphene on hexagonal boron nitride. Physical review letters 2016, 117(3), 036802.

[55] Hirai, H.; Tsuchiya, H.; Kamakura, Y.; Mori, N.; Ogawa, M. Electron mobility calculation for graphene on substrates. Journal of Applied Physics 2014, 116(8), 083703-083706.

[56] Kamalakar, M. V.; Dankert, A.; Bergsten, J.; Ive, T.; Dash, S. P. Enhanced tunnel spin injection into graphene using chemical vapor deposited hexagonal boron nitride. Scientific Reports 2014, 4, 1-8.

[57] Lee, M.; Wallbank, J. R.; Gallagher, P.; Watanabe, K.; Taniguchi, T.; Fal'ko, V. I.; Goldhaber-Gordon, D. Ballistic miniband conduction in a graphene superlattice. Science 2016, 353(6307), 1526-1529.

[58] Uddin, M. A.; Glavin, N.; Singh, A.; Naguy, R.; Jespersen, M.; Voevodin, A.; Koley, G. Mobility enhancement in graphene transistors on low temperature pulsed laser deposited boron nitride. Applied Physics Letters 2015, 107(20), 203110-203115. [59] Song, Y.; Zhang, C.; Li, B.; Ding, G.; Jiang, D.; Wang, H.; Xie, X. Van der Waals epitaxy and characterization of hexagonal boron nitride nanosheets on graphene. Nanoscale Research Letters 2014, 9(1), 1-7. 
[60] Khan, A. F.; Down, M. P.; Smith, G. C.; Foster, C. W.; Banks, C. E. Surfactant-exfoliated 2D hexagonal boron nitride (2D-hBN): role of surfactant upon the electrochemical reduction of oxygen and capacitance applications. Journal of Materials Chemistry A 2017, 5(8), 4103-4113. 



\section{Chapter 5}

One-step preparation of large area films of oriented $\mathrm{MoS}_{2}$ nanoparticles on multilayer graphene and its electrocatalytic activity for hydrogen evolution

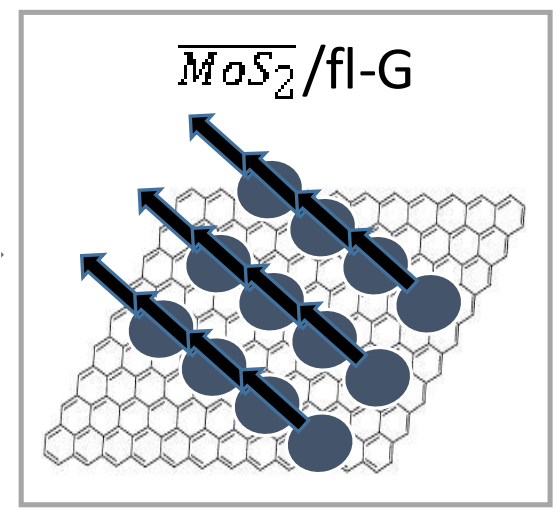





\subsection{Introduction}

Molybdenum disulfide $\left(\mathrm{MoS}_{2}\right)$ is among the most efficient non-noble metal electrocatalysts for water oxidation and reduction, and it has been proposed as an economically-viable alternative to the use of platinum in electrocatalysis for water splitting. ${ }^{[1-3]} \mathrm{MoS}_{2}$ is a 2D layered material that upon exfoliation can be supported on $\mathrm{G}$, and in this way, electrodes with high performance for hydrogen generation from water have been developed. ${ }^{[4,5]}$ It has been found that the electrocatalytic activity for the hydrogen evolution reaction of $\mathrm{MoS}_{2}$ increases as the particles become thinner and with the presence of steps, edges and defects on the particles. ${ }^{[4,5]}$ The most active sites on $\mathrm{MoS}_{2}$ have been proposed $\mathrm{S}$ vacancies at steps, followed by $\mathrm{S}$ vacancies on terraces and crystal planes. ${ }^{[4,5]}$

Our previous works have showed an innovative method for the preparation of hybrid $\mathrm{MoS}_{2} / \mathrm{G}$ materials as powders. ${ }^{[6]}$ The method is based on the simultaneous formation of $\mathrm{G}$ and $\mathrm{MoS}_{2}$ by pyrolysis at temperatures above $900{ }^{\circ} \mathrm{C}$ of a mixture of alginate containing $\left(\mathrm{NH}_{4}\right)_{2} \mathrm{MoS}_{4}$. As already commented it is sufficiently known that the pyrolysis of certain natural polysaccharides such as alginate and chitosan gives rise to the formation of turbostratic graphitic carbon residues that can be easily exfoliated with high efficiency to form $\mathrm{G}$ suspensions. ${ }^{[7]}$ Since these polysaccharides are good adsorbents, if the biopolymer contains metal ions or some other component, spontaneous phase segregation during the pyrolysis may lead to the formation of $\mathrm{G}$ having metal nanoparticles (NPs) or some other metal compound present on the $\mathrm{G}$ surface. ${ }^{[8-10]}$ This was the case of powders of alginate containing $\left(\mathrm{NH}_{4}\right)_{2} \mathrm{MoS}_{4}$ that upon pyrolysis and subsequent exfoliation of the carbon residue led to the formation of $\mathrm{MoS}_{2}$ platelets deposited on $\mathrm{G}$ by carbochemical reduction of $\mathrm{Mo}^{\mathrm{VI}}$ to $\mathrm{Mo}^{\mathrm{IV}}$ under the conditions of the pyrolysis. ${ }^{[6]}$ The simultaneous formation of $M_{0} S_{2}$ and $G$ in the process was assessed by XRD and Raman spectroscopy of the powders, recording the characteristic signature of both materials and also by transmission electron microscopy (TEM) and atomic force microscope (AFM) images 
of the resulting particles in suspension upon sonication of the powders. ${ }^{[6]}$ The $\mathrm{MoS}_{2} / \mathrm{G}$ material suspended in water was used as the catalyst for the visible light hydrogen generation using Eosin $\mathrm{Y}$ as the photosensitizer and methanol as the sacrificial electron donor. ${ }^{[6]}$

We have already presented that besides as powders, some of these natural polysaccharides such as alginates and chitosan are able to form defectless films of nanometric thickness and subnanometric rugosity that upon pyrolysis are converted into films of $\mathrm{G}$ or few-layer $\mathrm{G}^{[11]}$ The ability of some of these natural biopolymers to form films of nanometric thickness is directly responsible for the generation of the corresponding single or few-layer $G$ films upon graphitization. When the film of polysaccharide contains some adsorbed metal ion that does not form metal carbides, such as $\mathrm{Au}, \mathrm{Pt}$ and $\mathrm{Cu}$, their pyrolysis leads to the formation of $\mathrm{G}$ films having strongly anchored metal NPs exposing in several cases some preferential facet orientation. ${ }^{[8-10,12]}$ Continuing with this line of research, it would be of interest to exploit further this innovative film preparation procedure based on the pyrolysis of polysaccharide precursors for the one-step preparation of films of $\mathrm{MoS}_{2}$ supported on few-layer $\mathrm{G}$ that could be used directly as electrodes and determine their activity as electrocatalysts for hydrogen generation from water.

The present Chapter presents this type of electrocatalytic measurements on large area films $\left(2 \times 2 \mathrm{~cm}^{2}\right)$ of $\mathrm{MoS}_{2}$ supported on $\mathrm{G}$ prepared by pyrolysis of films of ammonium alginate containing adsorbed $\left(\mathrm{NH}_{4}\right)_{2} \mathrm{MoS}_{4}$. It will be shown here that as a consequence of the preparation procedure, the resulting $\mathrm{MoS}_{2}$ particles exhibit a preferential 002 facet orientation and present electrocatalytic activity for $\mathrm{H}_{2}$ evolution without the need for an additional conductive electrode, thus, representing a competitive advantage respect to the current $\mathrm{MoS}_{2}$ based electrocatalysts that require the presence of a conductive electrode to allow measurable current densities. 


\subsection{Results and discussion}

\subsubsection{Sample preparation and characterization}

Scheme 5.1 illustrates the preparation procedure of $\overline{\mathrm{MoS}_{2}} / \mathrm{ml}-\mathrm{G}\left(\overline{\mathrm{MoS}_{2}}\right.$ meaning 002 oriented $\mathrm{MoS}_{2}, \mathrm{ml}$ meaning multilayer). The process starts by dissolving alginic acid in an aqueous solution with the aid of ammonia. To this ammonium alginate solution, the required amount of $\left(\mathrm{NH}_{4}\right)_{2} \mathrm{MoS}_{4}$ was added. These solutions containing $\left(\mathrm{NH}_{4}\right)_{2} \mathrm{MoS}_{4}$ adsorbed on ammonium alginate were spin cast on a clean glass substrate, and the resulting ammonium alginate film was pyrolyzed under $\mathrm{Ar}$ at $900{ }^{\circ} \mathrm{C}$ (experimental details are provided in Chapter 8). Several films, where the loading of $\left(\mathrm{NH}_{4}\right)_{2} \mathrm{MoS}_{4}$ was varied by using initial $\left(\mathrm{NH}_{4}\right)_{2} \mathrm{MoS}_{4}$ concentrations in the range from 1 to $60 \mathrm{mM}$, were used. This process resulted in a set of $\overline{\mathrm{MoS}_{2}} / \mathrm{ml}-\mathrm{G}-\chi$ samples, where the $\chi$ value denotes the initial $\left(\mathrm{NH}_{4}\right)_{2} \mathrm{MoS}_{4}$ concentration used in the preparation of the films.

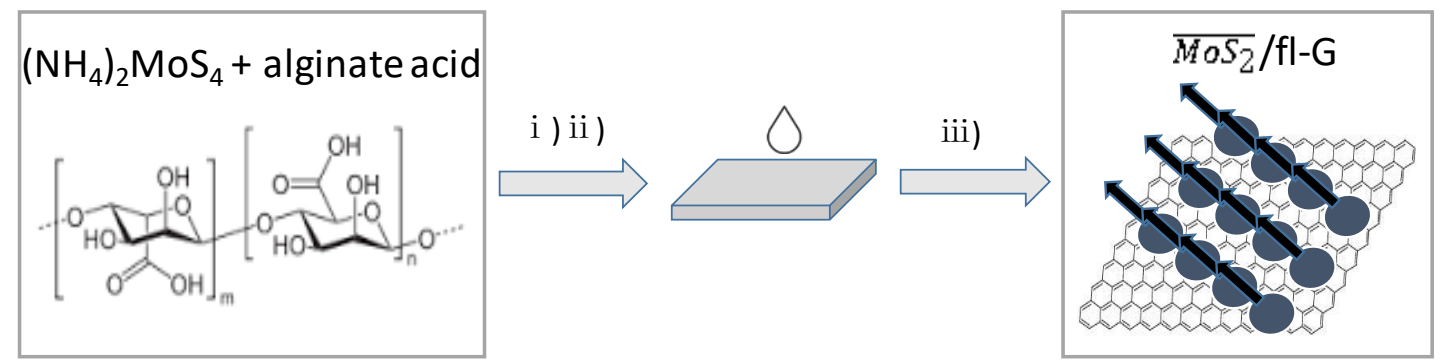

Scheme 5.1. Preparation procedure for $\overline{\operatorname{MoS}_{2}} / \mathrm{ml}-\mathrm{G}$ films supported on quartz:

(i) filtration of the aqueous solution of ammonium alginate containing $\left(\mathrm{NH}_{4}\right)_{2} \mathrm{MoS}_{4}$;

(ii) spin coating of alginate solution on clean quartz, and (iii) pyrolysis at $900{ }^{\circ} \mathrm{C}$ under Ar atmosphere.

The films resulting after the pyrolysis were initially characterized by XRD. As expected in view of related precedents, only the samples prepared with the highest $\left(\mathrm{NH}_{4}\right)_{2} \mathrm{MoS}_{4}$ concentrations showed some XRD peaks characteristic of $\mathrm{MoS}_{2}$. Figure 
5.1 presents the XRD pattern recorded for commercial MoS 2 powder and for a sample prepared using $\left(\mathrm{NH}_{4}\right)_{2} \mathrm{MoS}_{4}$ as the precursor with a concentration of $60 \mathrm{mM}$, where in addition to a broad band corresponding to graphitic carbon (multilayer G) of about $24^{\circ}$, a reflection at $14^{\circ}$ corresponding to the 002 diffraction of $\mathrm{MoS}_{2}$ could also be recorded. No other diffraction peaks expected also for $\mathrm{MoS}_{2}$ crystallites were present. The absence of the peaks corresponding to other diffraction planes indicates that $\mathrm{MoS}_{2}$ particles are formed exhibiting a preferential 002 facet orientation. Earlier precedents have shown that pyrolysis of thin films of alginate and chitosan containing $\mathrm{Au}, \mathrm{Pt}, \mathrm{Ag}$ and $\mathrm{Cu}$ among other metals can result in metal nanoplatelets supported on $\mathrm{G}$ exhibiting preferential 111 facet orientation. ${ }^{[8,10,12]}$ The case presented in this Chapter constitutes an additional example of the formation of nanoparticles with a preferential crystallographic orientation, the 002 facet in the $\mathrm{MoS}_{2}$ particles. In accordance with earlier proposals, the most likely reasons for this preferential particle growth are the template effect of graphene layer on the nascent $\mathrm{MoS}_{2}$ nanoparticles and/or the higher thermodynamic stability of the 002 surface respect to other crystal facets.

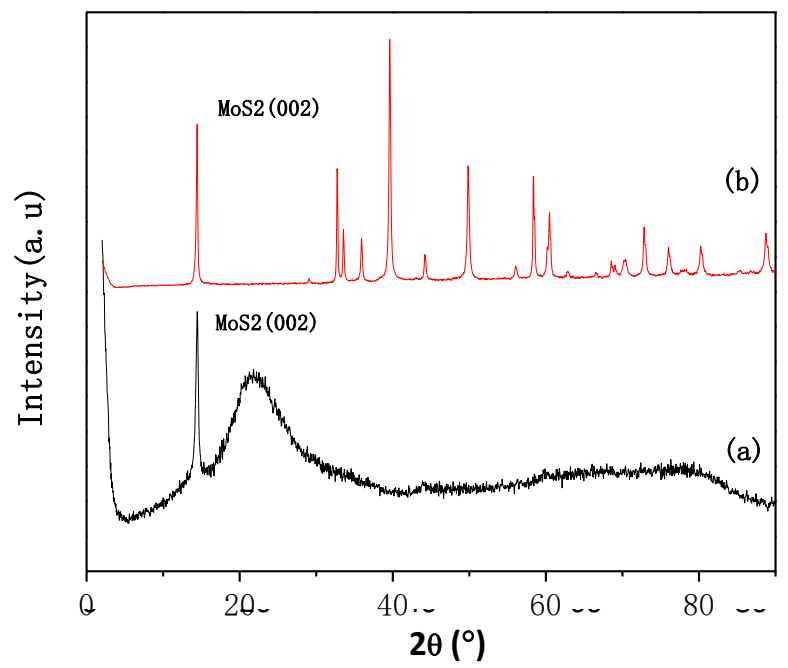

Figure 5.1. XRD patterns of $\overline{\mathrm{MoS}_{2}} / \mathrm{ml}-\mathrm{G}-60$ film (a) and the commercial $\mathrm{MoS}_{2}$ powder (b).

The morphology of $\mathrm{MoS}_{2}$ nanoplatelets and their average particle size on the samples were determined by scanning electron microscopy (SEM). Figure 5.2 
presents the images of the films prepared with initial $\left(\mathrm{NH}_{4}\right)_{2} \mathrm{MoS}_{4}$ concentrations of $0.5,2,5$ and $10 \mathrm{mM}$, showing that the $\overline{\mathrm{MoS}_{2}} / \mathrm{ml}-\mathrm{G}$ samples contain particles of $\mathrm{MoS}_{2}$ distributed homogeneously all over the G film with a broad particle size distribution ranging approximately from 10-200 $\mathrm{nm}$. Quantitative analysis of the particles observed in the SEM images by energy dispersive X-ray spectroscopy (EDX) confirmed that these particles are constituted by Mo and S in an approximate atomic 1:2 ratio, providing firm evidence of the composition of the particles seen in the images. The most important conclusion of this SEM study is that there was a clear relationship between the average $\mathrm{MoS}_{2}$ particle size and the concentration of $\left(\mathrm{NH}_{4}\right)_{2} \mathrm{MoS}_{4}$ used in the preparation, the average particle size increasing along the concentration of $\left(\mathrm{NH}_{4}\right)_{2} \mathrm{MoS}_{4}$ used in the preparation of the $\overline{\mathrm{MoS}_{2}} / \mathrm{ml}-\mathrm{G}$ films. In this way, the $\overline{\mathrm{MoS}_{2}} / \mathrm{ml}-\mathrm{G}-\mathrm{x}$ with the smallest average particle size of $15 \mathrm{~nm}$ corresponded to $\overline{\operatorname{MoS}_{2}} / \mathrm{ml}-\mathrm{G}-0.5$, while the average particle size grows to 37,78 and $105 \mathrm{~nm}$ for $\overline{\mathrm{MoS}_{2}} / \mathrm{ml}-\mathrm{G}-2, \overline{\mathrm{MoS}_{2}} / \mathrm{ml}-\mathrm{G}-5$ and $\overline{\mathrm{MoS}_{2}} / \mathrm{ml}-\mathrm{G}-10$, respectively. It is known that an increase in particle size has generally a detrimental influence on the electrocatalytic performance that decreases as the particles become larger.

The synthesized samples composed of $\mathrm{MoS}_{2}$ nanoplates embedded in graphene are quite different with the $G(N) / h-B N$ heterostructures in Chapter 4 where segregated layers of $\mathrm{h}-\mathrm{BN}$ and graphene were formed, as the similar preparation methods for these two materials were used. Actually, it could be due to the different contributions of graphene to the synthetic process of these two heterostructures. For the $(\mathrm{N}) \mathrm{G} / \mathrm{h}-\mathrm{BN}$ heterostructures, the $\mathrm{N}$-doped graphene behaved more like an internal template where $\mathrm{B}, \mathrm{N}$ gradually doped into the layer structure and this finally resulted in the formation of segregated h-BN layer based on the graphene sheet. However, for the graphene/MoS 2 materials, the graphene behaved more like an external template where $\mathrm{MoS}_{2}$ were formed on the surface of the hexagonal structure. This contributed to the oriented growth of $\mathrm{MoS}_{2}$ nanoplates on the graphene layer and led to the formation of a composite of $\mathrm{MoS}_{2}$ nanoplates embedded in graphene. 

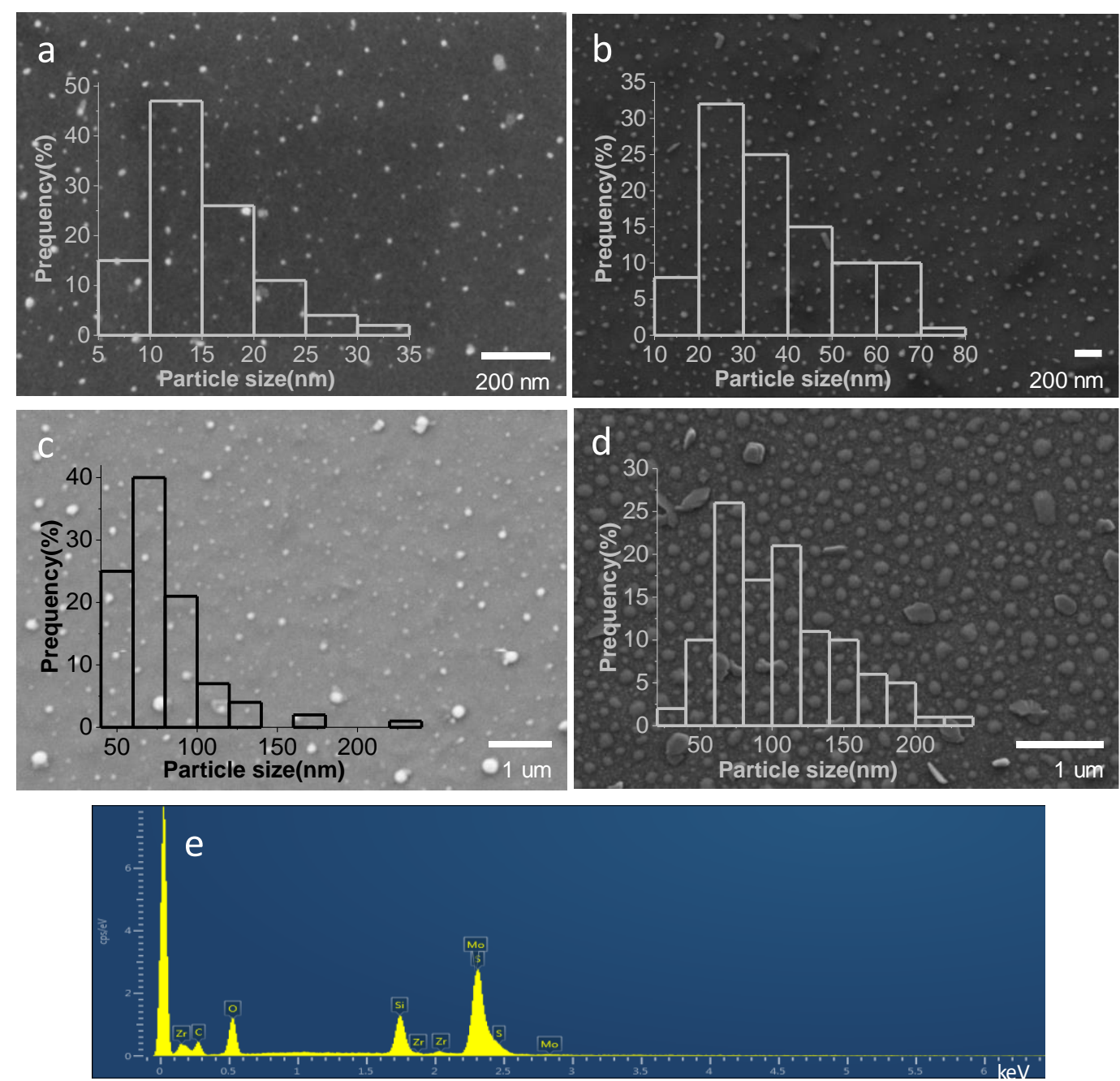

Figure 5.2. Representative SEM images of $\overline{\operatorname{MoS}_{2}} / \mathrm{ml}-\mathrm{G}-0.5$ (a), $\overline{\mathrm{MoS}_{2}} / \mathrm{ml}-\mathrm{G}-2$ (b), $\overline{\operatorname{MoS}_{2}} / \mathrm{ml}-\mathrm{G}-5$ (c), $\overline{\operatorname{MoS}_{2}} / \mathrm{ml}$-G-10 (d), as well as the different histograms of $\mathrm{MoS}_{2}$ particle size distribution and the EDX analysis of the $\overline{\mathrm{MoS}_{2}} / \mathrm{ml}-\mathrm{G}-2$ (e) confirming that the particles correspond to $\mathrm{MoS}_{2}$ supported on $\mathrm{ml}-\mathrm{G}$. Note that the presence of $\mathrm{Zr}$ and $\mathrm{Si}$ is due to the sample holder used for SEM and the quartz substrate, respectively.

Unfortunately, TEM images of the $\overline{\mathrm{MoS}_{2}} / \mathrm{ml}-\mathrm{G}$ films cannot be obtained without detaching them from the quartz substrate. Accordingly, TEM images of the $\overline{\mathrm{MoS}_{2}} / \mathrm{ml}-\mathrm{G}-2$ could only be obtained after scratching debris of the $\overline{\mathrm{MoS}_{2}} / \mathrm{ml}-\mathrm{G}$ film from the quartz substrate. Figure 5.3 provides a set of TEM images of pieces of the $\overline{\mathrm{MoS}_{2}} / \mathrm{ml}$-G film detached from the quartz substrate. Figure 5.3a shows a wide view 
image of the $\overline{\mathrm{MoS}_{2}} / \mathrm{ml}$-G film showing the presence of $\overline{\mathrm{MoS}_{2}}$ particles (darker particles) surrounded by graphene characterized by lighter contrast. Figure 5.3(b,c) focuses on $\overline{\mathrm{MoS}_{2}}$ particles. In Panel c, the presence of graphene layers (lighter contrast) wrapping the $\mathrm{MoS}_{2}$ particle is clearly observed. High resolution TEM measurements of the interlayer distance of the particles give a value of $0.62 \mathrm{~nm}$, which agrees with the value for the 002 interplanar distance in $\mathrm{MoS}_{2}$, thus providing an additional confirmation of the preferential 002 orientation of the $\mathrm{MoS}_{2}$ particles determined by XRD for those samples with high $\mathrm{MoS}_{2}$ loading. ${ }^{[13]}$ Fast Fourier transformed (FFT) electron diffraction showed that the $\mathrm{MoS}_{2}$ particles were highly crystalline. Figure 5.3 shows three selected TEM images at different magnifications, as well as the measurement of the interplanar distance and the FFT electron diffraction pattern taken from the image.

By using the AFM technique with subnanometric vertical resolution, the thickness of the $\overline{\mathrm{MoS}_{2}} / \mathrm{ml}-\mathrm{G}-2$ film and the nanoplatelet morphology of $\mathrm{MoS}_{2}$ particles grafted on $\mathrm{G}$ were confirmed. Figure 5.4 presents the measurement of the heights for three representative large $\mathrm{MoS}_{2}$ nanoplatelets with a lateral area of about $80 \mathrm{~nm}$, showing that these particles are flat and thin, with heights between 5 and $15 \mathrm{~nm}$. The thickness of the graphene film could also be measured at the edge of the scratch. It was determined that the average thickness of $\overline{\mathrm{MoS}_{2}} / \mathrm{ml}-\mathrm{G}-2$ film was about $20 \mathrm{~nm}$ (Figure 4d). 


\section{Chapter 5}
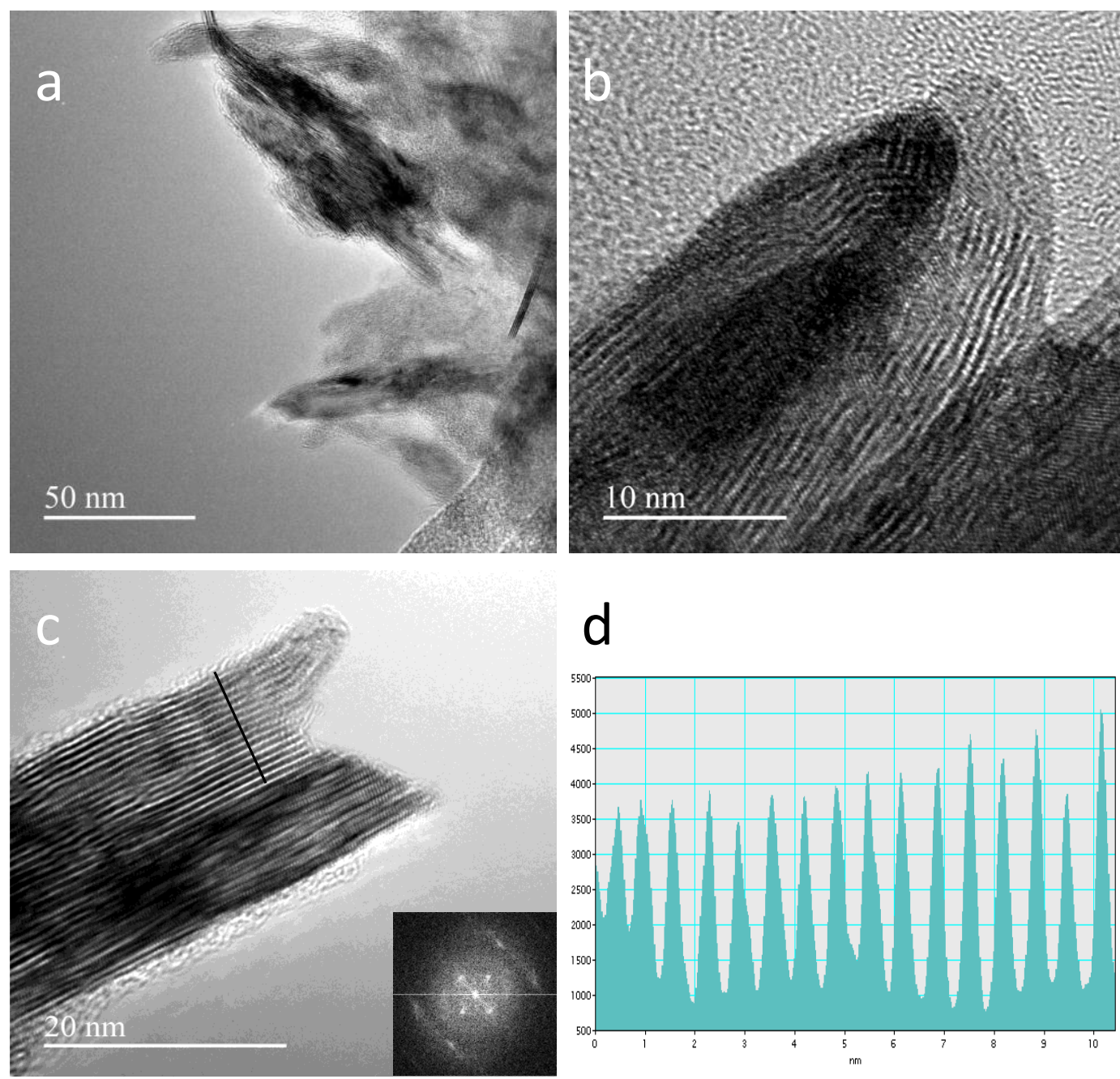

\section{d}

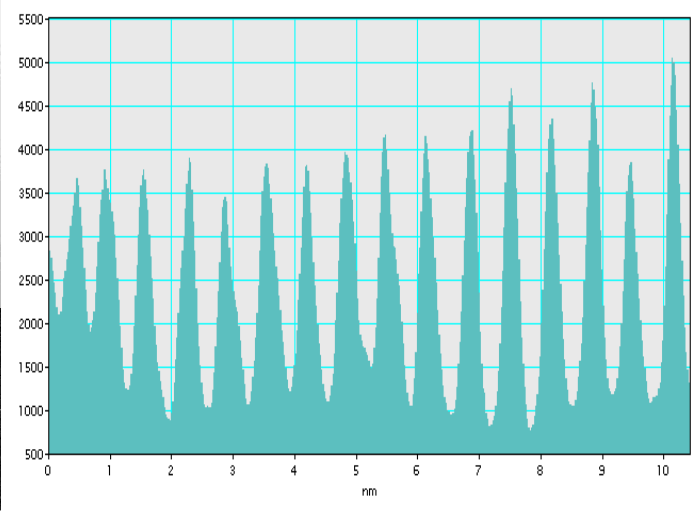

Figure 5.3. TEM images at different magnifications of $\overline{\mathrm{MoS}_{2}} / \mathrm{ml}-\mathrm{G}-2$ after scratching the film from the quartz substrate $(\mathbf{a}, \mathbf{b}, \mathbf{c})$. Image a shows a general view where the presence of $\mathrm{MoS}_{2}$ as darker particles can be seen embedded within the multilayer graphene film in lighter contrast. The inset of panel c shows the FFT electron diffraction pattern, and image $\mathbf{d}$ shows the measurement of the distance between different planes. 
Chapter 5
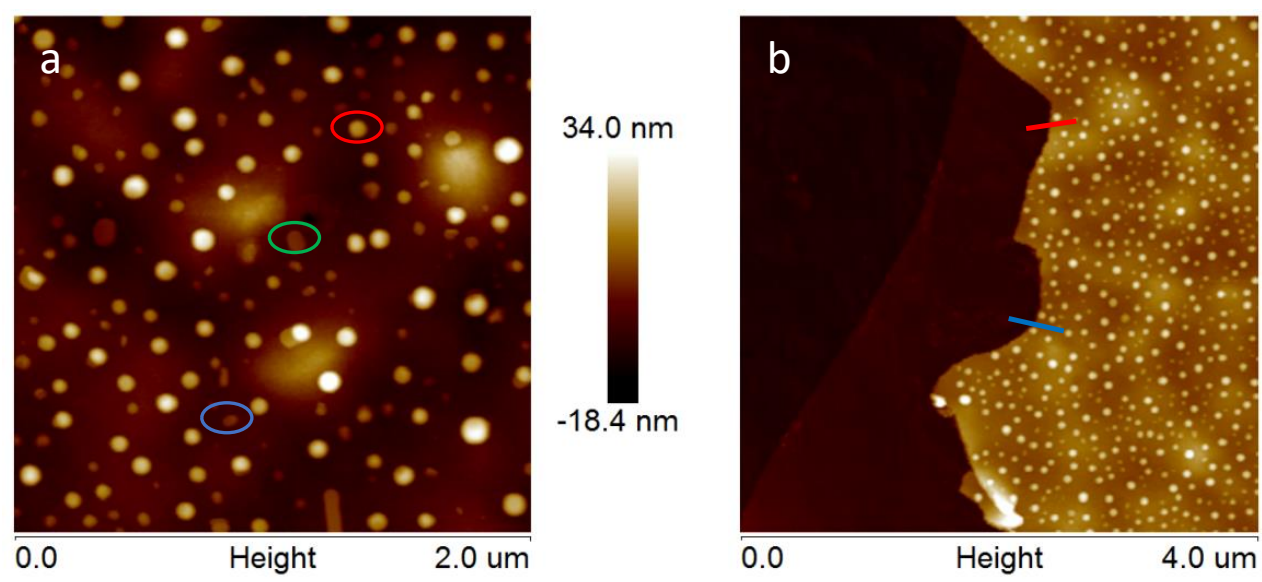

$34.7 \mathrm{~nm}$

$-51.3 \mathrm{~nm}$
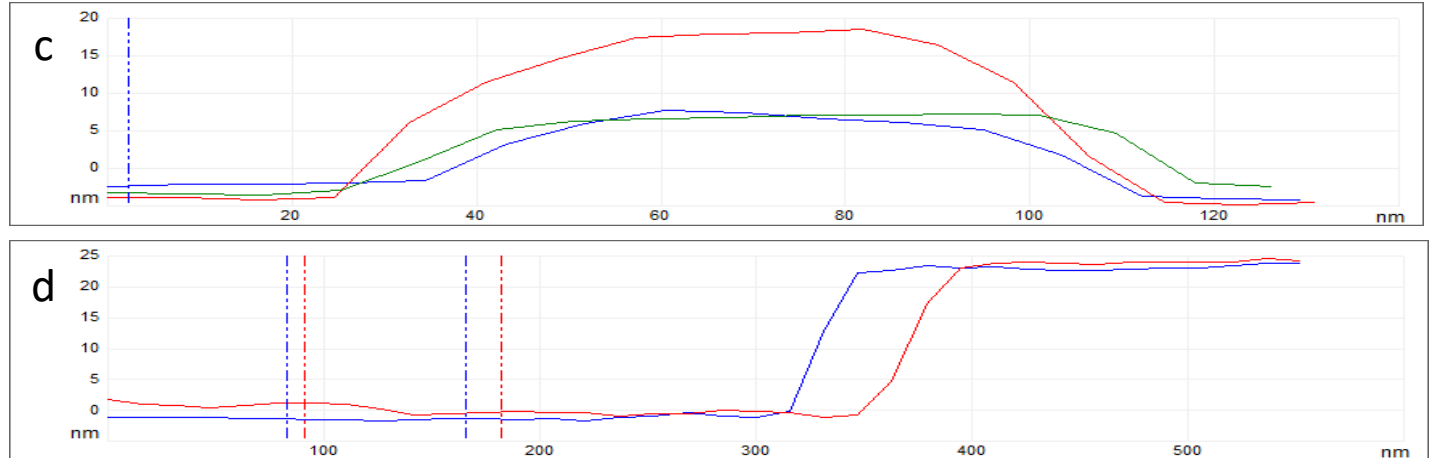

Figure 5.4. Top views of the AFM images at the central (a) and border (b) part of the $\overline{\operatorname{MoS}_{2}} / \mathrm{ml}-\mathrm{G}-2$ sample. Frame c shows the height and lateral dimensions of three representative $\mathrm{MoS}_{2}$ nanoparticles marked in the image with the corresponding red, green and blue color. Frame $\mathbf{d}$ shows the section profile at two points of the edge of the film (red and blue lines in panel b) from which it can be determined that the thickness of the $\mathrm{ml}-\mathrm{G}$ film is about $20 \mathrm{~nm}$.

Raman spectra of $\overline{\mathrm{MoS}_{2}} / \mathrm{ml}-\mathrm{G}$ samples show the characteristic $2 \mathrm{D}, \mathrm{G}$ and $\mathrm{D}$ peaks appearing at 2912, 1602 and $1367 \mathrm{~cm}^{-1}$, respectively, expected for defective G. As an example, Figure 5.5 shows the Raman spectra for $\overline{\mathrm{MoS}_{2}} / \mathrm{ml}-\mathrm{G}-2$ film. The intensity of the $G$ vs. the $D$ band $\left(I_{G} / I_{D}\right)$ gives a quantitative indication of the density of defects of the $G$ layers. In the present case, the $I_{G} / I_{D}$ value is about 1.26 , which is higher than those $I_{G} / I_{D}$ values typically reported for reduced graphene oxide ( $\mathrm{rGO}$ ) samples, which are generally about $0.9 .^{[14]}$ This indicates that $\mathrm{G}$ in the $\overline{\mathrm{MoS}_{2}} / \mathrm{ml}-\mathrm{G}$ samples should have somewhat lower defect density than conventional rGO samples. No peaks due to the presence of $\mathrm{MoS}_{2}$ could be observed. $\mathrm{MoS}_{2}$ exhibits in 
Raman spectroscopy two characteristic $\mathrm{A} 1 \mathrm{~g}$ and E1g vibration modes at about 400 and $380 \mathrm{~cm}^{-1}[15]$ that could not be recorded in the present $\overline{M_{0} S_{2}} / \mathrm{ml}-\mathrm{G}$ films. The low $\mathrm{MoS}_{2}$ loading, together with the low intensity of their Raman bands are the two most probable reasons for the lack of $\mathrm{MoS}_{2}$ detection, as was already discussed when commenting on the XRD patterns that could be only observed for the $\overline{\mathrm{MoS}_{2}} / \mathrm{ml}-\mathrm{G}$ sample prepared at the highest $\left(\mathrm{NH}_{4}\right)_{2} \mathrm{MoS}_{4}$ concentration.

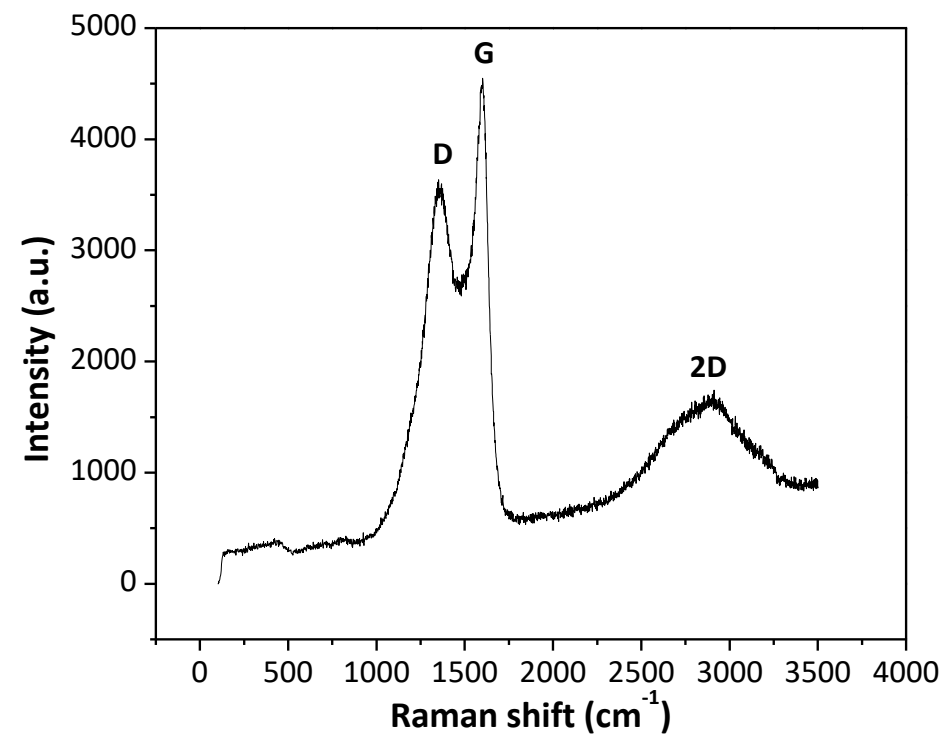

Figure 5.5. Raman spectrum of the $\overline{\mathrm{MoS}_{2}} / \mathrm{ml}-\mathrm{G}-2$ sample.

The chemical states of Mo, S and $\mathrm{C}$ in the $\overline{\mathrm{MoS}_{2}} / \mathrm{ml}-\mathrm{G}$ sample and the distribution among various coordination environments were investigated by carrying out the X-ray photoelectron spectroscopy (XPS) measurements (Figure 5.6). The survey XPS spectrum of $\overline{\mathrm{MoS}_{2}} / \mathrm{ml}-\mathrm{G}$ film shows the presence of the expected $\mathrm{C}, \mathrm{O}$, Mo and S elements, the latter two with very low intensity. In addition to the lower response factor of these two elements with respect to $C$ and $O$, the low intensity of Mo and $S$ peaks could indicate that $\overline{M_{0 S}}$ particles are not well exposed to the external surface and that they could be partially wrapped by G layers. 

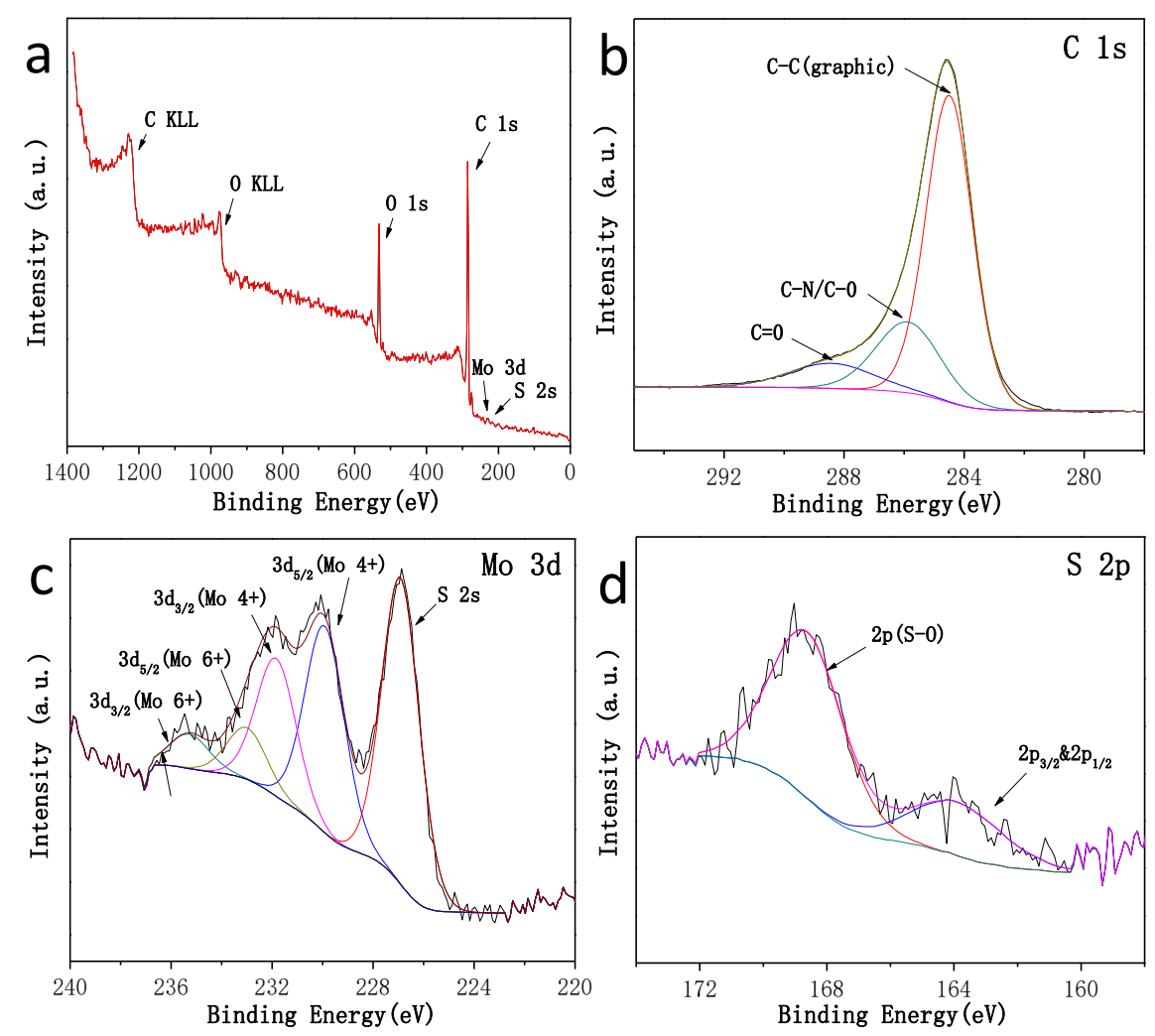

Figure 5.6. High-resolution XPS spectra of the $\overline{\mathrm{MoS}_{2}} / \mathrm{ml}-\mathrm{G}-2$ material. (a) Survey spectrum; (b) C 1s spectrum; (c) Mo 3d spectrum and S 2s; (d) region corresponding to the $S 2 p$ peak. Note that Mo and the main S $2 s$ peaks appear in $(\mathbf{c})$, where some components corresponding to Mo $(6+)$ have been marked for clarity.

The high resolution XPS spectra show that the $C$ 1s peak can be resolved into three peaks centered at 284.5 (68.1\%), 285.9 (10.2\%) and $288.4 \mathrm{eV}(21.7 \%)$, which could correspond to graphitic carbons, $\mathrm{C}-\mathrm{O} / \mathrm{C}-\mathrm{N}$ and $\mathrm{C}=\mathrm{O}$, respectively. The $\mathrm{Mo} 3 \mathrm{~d}$ spectrum spectra shows the existence of the Mo (4+) oxidation state $(73.4 \%)$, as well as the Mo $(6+)$ oxidation state $(26.6 \%)$, the latter probably due to the formation of some $\mathrm{MoO}_{3}$ (about $20 \%$ ) on the surface of the $\mathrm{MoS}_{2}$ nanoparticles upon exposure of the sample to air. ${ }^{[16]}$ It should be noted that the presence of some $\mathrm{MoO}_{3}$ only corresponds to the outermost surface of the sample probed by XPS and that elemental analysis by SEM confirms the $\mathrm{MoS}_{2}$ stoichiometry. The presence of a high proportion of $\mathrm{MoS}_{2}$ on the surface of the material is also confirmed by the observation of the corresponding S 2s peak at $226.5 \mathrm{eV}$ binding energy, appearing 
near to the Mo peak (Figure 5.6c) corresponding to about $80 \%$ of all the $\mathrm{S}$ atoms. The presence of some S-O band (18\%) and a small amount of bridging $\mathrm{S}_{2}{ }^{2-}(4 \%)$ were also detected in a different binding energy value region by the corresponding $S$ $2 p$ at 164.0 and $163.2 \mathrm{eV}$ (Figure $5.6 \mathrm{~d}) .{ }^{[17]}$

\subsubsection{Electrocatalytic measurements}

Square $\overline{\mathrm{MoS}_{2}} / \mathrm{ml}-\mathrm{G}$ films supported on quartz of a surface of $2 \times 2 \mathrm{~cm}^{2}$ resulting from the pyrolysis of alginate precursors were directly used as electrodes for $\mathrm{H}_{2}$ generation. Note that $\overline{\mathrm{MoS}_{2}} / \mathrm{ml}-\mathrm{G}$ films are not coating the glassy carbon electrode or any other conductive substrate and that the electrical conductivity in $\overline{\mathrm{MoS}_{2}} / \mathrm{ml}-\mathrm{G}$ derives from the intrinsic properties of the multilayer graphene forming the film. Previous reports in the literature have established that films of defective Gs obtained by pyrolysis of natural polysaccharides on quartz substrates exhibit notable electrical conductivity, with surface resistivity values in the range of a few $\mathrm{k} \Omega / \mathrm{sq}$ (sq meaning square). ${ }^{[7,11]}$ The fact that no conductive electrode is needed in the case of $\overline{\mathrm{MoS}_{2}} / \mathrm{ml}-\mathrm{G}$ is one important advantage derived from the preparation procedure and from the composition of the samples.

Representative measurements of the electrocatalytic behavior of the $\overline{\mathrm{MoS}_{2}} / \mathrm{ml}-\mathrm{G}$ films for $\mathrm{H}_{2}$ generation are presented in Figure 5.7, where the performance of $\overline{\mathrm{MoS}_{2}} / \mathrm{ml}-\mathrm{G}$ films is compared to that of Pt nanoparticles deposited on glassy carbon. As can be seen there, differences in the onset for $\mathrm{H}_{2}$ generation and in the current density of the $\overline{\mathrm{MoS}_{2}} / \mathrm{ml}-\mathrm{G}$ electrodes as a function of the concentration of $\left(\mathrm{NH}_{4}\right)_{2} \mathrm{MoS}_{4}$ used in the preparation of the electrodes were observed, there being an optimal loading corresponding to $\left(\mathrm{NH}_{4}\right)_{2} \mathrm{MoS}_{4}$ close to 2 $\mathrm{mM}$. The observation of an optimal loading typically occurs when there are two opposite factors related to the amount of $\mathrm{MoS}_{2}$ deposited on the support influencing the electrocatalytic activity. We propose that these two opposite factors are: i) the catalytic activity of $\mathrm{MoS}_{2}$ for $\mathrm{H}_{2}$ evolution that should increase as the loading of $\mathrm{MoS}_{2}$ increases, and ii) the increase in the particle size of $\mathrm{MoS}_{2}$ with lesser density of defects that should disfavor the catalytic activity as $\mathrm{MoS}_{2}$ loading increases. As 
commented at the beginning of this Chapter, it is known that the electrocatalytic activity of $\mathrm{MoS}_{2}$ derives from steps and defects on the nanoparticles, ${ }^{[18,19]}$ and these defects should be more abundant when the particle size is smaller, a fact that should occur at low $\mathrm{MoS}_{2}$ loadings. As mentioned earlier, SEM images clearly indicate that the particle size grows from 15-105 nm upon increasing $\left(\mathrm{NH}_{4}\right)_{2} \mathrm{MoS}_{4}$ concentration. On the other hand, for low $\mathrm{MoS}_{2}$ loadings, the density of active sites in $2 \times 2 \mathrm{~cm}^{2}$ should be low, resulting in low activity, as it was actually the case of the $\overline{\mathrm{MoS}_{2}} / \mathrm{ml}-\mathrm{G}-1$ film prepared using the $0.5 \mathrm{M}\left(\mathrm{NH}_{4}\right)_{2} \mathrm{MoS}_{4}$ concentration. As a result, a compromise should be reached at an optimal $\mathrm{MoS}_{2}$ loading close to a $2 \mathrm{mM}$ $\left(\mathrm{NH}_{4}\right)_{2} \mathrm{MoS}_{4}$ concentration during the preparation of the $\overline{\mathrm{MoS}_{2}} / \mathrm{ml}-\mathrm{G}$ films.

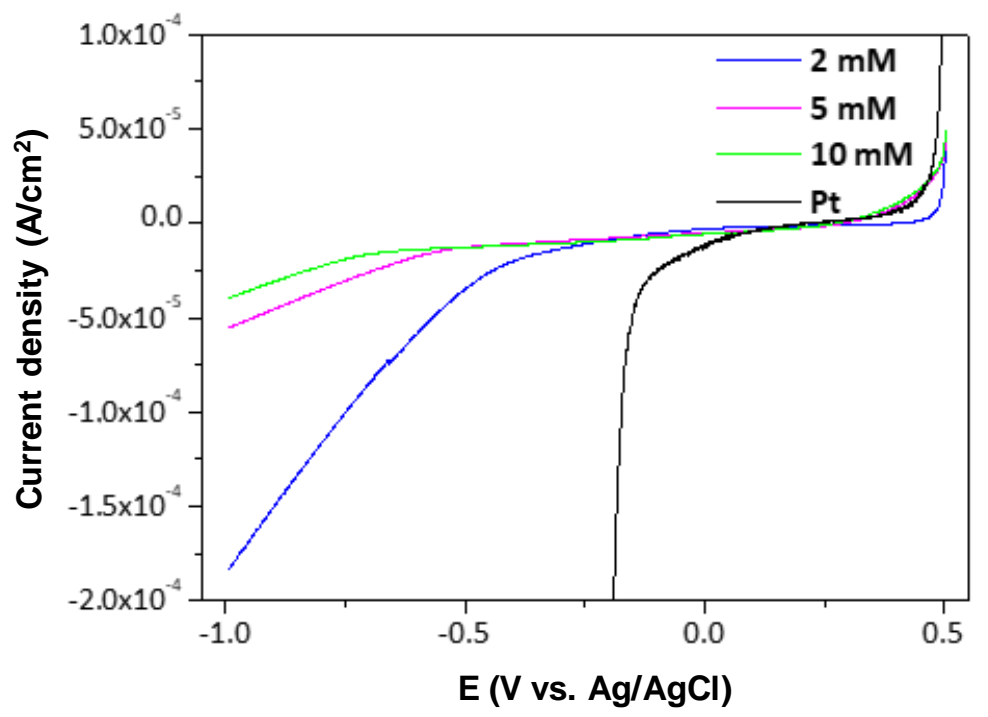

Figure 5.7. Polarization curves of the $\overline{\mathrm{MoS}_{2}} / \mathrm{ml}-\mathrm{G}$ films and Pt nanoparticles on glassy carbon electrode for hydrogen evolution reaction (HER) activity.

By performing a series of independent electrode preparations, it was observed that the electrocatalytic response of the $\overline{\mathrm{MoS}_{2}} / \mathrm{ml}-\mathrm{G}$ films was not exactly reproducible from one batch to the other, there being a dispersion on the potential onset of $\mathrm{H}_{2}$ generation and on the current density achieved at different potentials for the $\overline{\mathrm{MoS}_{2}} / \mathrm{ml}-\mathrm{G}$ films as a function of the $\left(\mathrm{NH}_{4}\right)_{2} \mathrm{MoS}_{4}$ concentration. Figure 5.8 presents data of three sets of $\overline{\mathrm{MoS}_{2}} / \mathrm{ml}-\mathrm{G}$ film prepared independently, showing the variability in the response of the electrodes. We suggest that this lack of complete 
reproducibility derives in a large extent from the difficulty to make electrical contacts on films of nanometric thickness and on the random growth of $\mathrm{MoS}_{2}$ particles during the pyrolysis, particularly in the low concentration range. In any case, whatever the reason, independent preparation of several series of $\overline{\mathrm{MoS}_{2}} / \mathrm{ml}-\mathrm{G}-\mathrm{X}$ films showed that the optimal concentration values were in the range between 1 and $2 \mathrm{mM}$ (see Figure 5.8), for which an onset potential of $-0.2 /-0.3 \mathrm{~V}$ is consistently measured, with Tafel slopes of $180 \mathrm{mV} /$ decade. Thus, the dispersion in the behavior of the electrodes, although existing, allows a degree of confidence on the performance of $\overline{\mathrm{MoS}_{2}} / \mathrm{ml}-\mathrm{G}$ films. In the literature, an onset potential for $\mathrm{MoS}_{2}$ supported on reduced graphene oxide deposited on a conductive glassy carbon electrode of $100 \mathrm{mV}$ with a rise of $41 \mathrm{mV} /$ decade was reported on a $0.5 \mathrm{M} \mathrm{H}_{2} \mathrm{SO}_{4}$ aqueous solution. ${ }^{[4]}$ Note, however, that although the electrolyte solutions in the reported data and the present study are the same, other conditions and, particularly, the absence of a conductive electrode and the use of flat quartz substrate as the electrode are remarkably different from those used in the literature. ${ }^{[4]}$

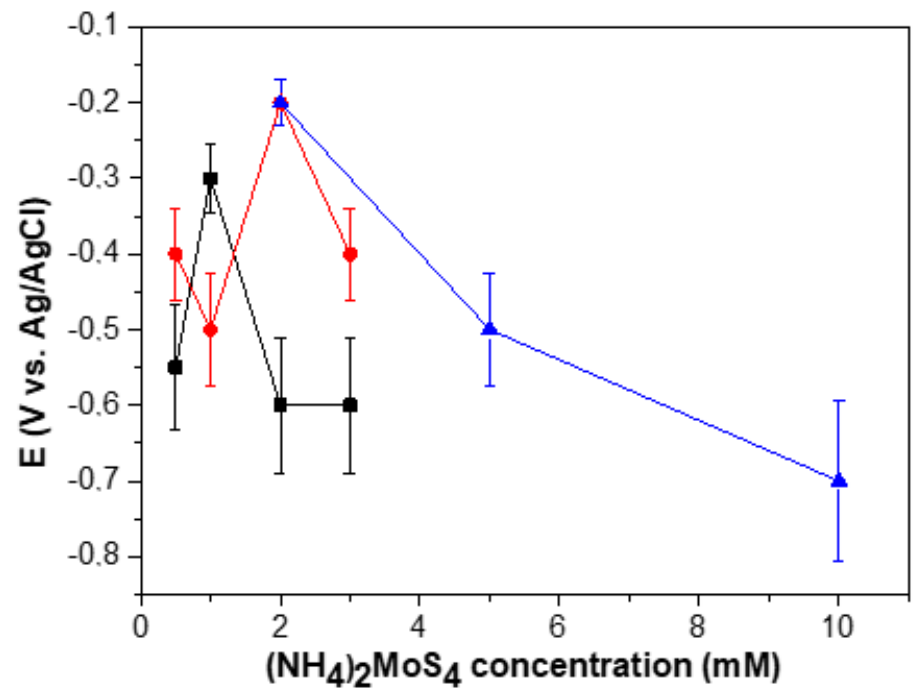

Figure 5.8. The electrocatalytic performance characterized by the onset potential for $\mathrm{H}_{2}$ evolution of three independent batches of samples (in different colors) as a function of the concentration of $\left(\mathrm{NH}_{4}\right)_{2} \mathrm{MoS}_{4}$ precursor.

Regarding stability, it was observed that $\overline{\mathrm{MoS}_{2}} / \mathrm{ml}-\mathrm{G}$ films undergo easily peeling off from the quartz substrate upon a few electrocatalytic measurements. This 
reflects poor adherence of $\overline{\mathrm{MoS}_{2}} / \mathrm{ml}-\mathrm{G}$ films to the quartz substrate. This detachment of the $\overline{\mathrm{MoS}_{2}} / \mathrm{ml}-\mathrm{G}$ films from the electrical contacts preclude a more realistic determination of the stability of $\overline{\mathrm{MoS}_{2}} / \mathrm{ml}-\mathrm{G}$ films as electrocatalysts for the $\mathrm{H}_{2}$ evolution reaction.

\subsection{Conclusions}

In the present Chapter, it is reported that large surface area films of $\overline{\mathrm{MoS}_{2}} / \mathrm{ml}-\mathrm{G}$ on arbitrary, non-conductive substrates can be prepared in one step by pyrolysis at $900{ }^{\circ} \mathrm{C}$ under $\mathrm{Ar}$ of ammonium alginate films containing $\left(\mathrm{NH}_{4}\right)_{2} \mathrm{MoS}_{4}$. During the pyrolytic process, two separate phases corresponding to graphitic carbon (multilayer graphene) and $\mathrm{MoS}_{2}$ develop spontaneously. $\mathrm{MoS}_{2}$ platelets exhibit a preferential 002 facet orientation and they have affinity for graphene as deduced from the relative lateral surface area to height ratio, which is large. The $\overline{M_{0} S_{2}} / \mathrm{ml}-\mathrm{G}$ films act as electrocatalysts for $\mathrm{H}_{2}$ generation without the need for any conductive electrode, exhibiting a potential onset between -0.2 and $-0.3 \mathrm{~V}$ depending on the concentration of $\left(\mathrm{NH}_{4}\right)_{2} \mathrm{MoS}_{4}$ used in the preparation, with certain variability on the electrocatalytic performance from batch to batch. Considering the simplicity of the one-step preparation procedure and the precursors, the present protocol is advantageous for the preparation of $\overline{\mathrm{MoS}_{2}} / \mathrm{ml}-\mathrm{G}$ films as electrocatalysts in an easily scalable way without the need of conductive electrode. 


\subsection{References}

[1] Chhowalla, M.; Shin, H. S.; Eda, G.; Li, L. J.; Loh, K. P.; Zhang, H. The chemistry of two-dimensional layered transition metal dichalcogenide nanosheets. Nature Chemistry 2013, 5(4), 263-275.

[2] Lukowski, M. A.; Daniel, A. S.; Meng, F.; Forticaux, A.; Li, L.; Jin, S. Enhanced hydrogen evolution catalysis from chemically exfoliated metallic $\operatorname{MoS}_{2}$ nanosheets. Journal of the American Chemical Society 2013, 135(28), 10274-10277.

[3] Voiry, D.; Yamaguchi, H.; Li, J.; Silva, R.; Alves, D. C.; Fujita, T.; Chen, M.; Asefa, T.; Shenoy, V. B.; Eda, G. Enhanced catalytic activity in strained chemically exfoliated $W_{2}$ nanosheets for hydrogen evolution. Nature Materials 2013, 12(9), 850-855.

[4] Li, Y.; Wang, H.; Xie, L.; Liang, Y.; Hong, G.; Dai, H. MoS 2 nanoparticles grown on graphene: An advanced catalyst for the hydrogen evolution reaction. Journal of the American Chemical Society 2011, 133(19), 7296-7299.

[5] Liao, L.; Zhu, J.; Bian, X.; Zhu, L.; Scanlon, M. D.; Girault, H. H.; Liu, B. MoS2 formed on mesoporous graphene as a highly active catalyst for hydrogen evolution. Advanced Functional Materials 2013, 23(42), 5326-5333.

[6] Latorre-Sanchez, M.; Esteve-Adell, I.; Primo, A.; Garcia, H. Innovative preparation of $\mathrm{MoS}_{2}$-graphene heterostructures based on alginate containing $\left(\mathrm{NH}_{4}\right)_{2} \mathrm{MoS}_{4}$ and their photocatalytic activity for $\mathrm{H}_{2}$ generation. Carbon 2015, 81, 587-596.

[7] Primo, A.; Sanchez, E.; Delgado, J. M.; Garcia, H. High-yield production of n-doped graphitic platelets by aqueous exfoliation of pyrolyzed chitosan. Carbon 2014, 68, 777-783.

[8] Mateo, D.; Esteve-Adell, I.; Albero, J.; Sanchez Royo, J. F.; Primo, A.; Garcia, H. 111 oriented gold nanoplatelets on multilayer graphene as visible light photocatalyst for overall water splitting. Nature Communications 2016, 7, 11819.

[9] Primo, A.; Esteve-Adell, I.; Blandez, J. F.; Dhakshinamoorthy, A.; Alvaro, M.; Candu, N.; Coman, S. M.; Parvulescu, V. I.; Garcia, H. High catalytic activity of oriented 2.0.0 copper(I) oxide grown on graphene film. Nature Communications 2015, 6, 8561. 
[10] Primo, A.; Esteve-Adell, I.; Coman, S. N.; Candu, N.; Parvulescu, V. I.; Garcia, H. One-step pyrolysis preparation of 1.1 .1 oriented gold nanoplatelets supported on graphene and six orders of magnitude enhancement of the resulting catalytic activity. Angewandte Chemie International Edition 2016, 128(2), 617-622.

[11] Primo, A.; Atienzar, P.; Sanchez, E.; Maria Delgado, J.; Garcia, H. From biomass wastes to large-area, high-quality, n-doped graphene: catalyst-free carbonization of chitosan coatings on arbitrary substrates. Chemical Communications 2012, 48(74), 9254-9256.

[12] Esteve-Adell, I.; Bakker, N.; Primo, A.; Hensen, E.; Garcia, H. Oriented Pt nanoparticles supported on few-layers graphene as highly active catalyst for aqueous-phase reforming of ethylene glycol. ACS Applied Materials \& Interfaces 2016, 8(49), 33690-33696.

[13] Pedraza, F.; Cruz-Reyes, J.; Acosta, D.; Yanez, M. J.; Avalos-Borja, M.; Fuentes, S. The structure characterization of $\mathrm{MoS}_{2}$ and WS 2 catalysts by HREM. Journal of Physics-Condensed Matter 1993, 5(33A), A219.

[14] Jorio, A.; Saito, R.; Dresselhaus, G.; Dresselhaus, M. S. Disorder effects in the raman spectra of $\mathrm{sp}^{2}$ carbons. Raman spectroscopy in graphene related systems 2011, 299-325.

[15] Li, H.; Zhang, Q.; Yap, C.; Tay, B. K.; Edwin, T.; Olivier, A.; Baillargeat, D. From bulk to monolayer $\mathrm{MoS}_{2}$ : evolution of Raman scattering. Advanced Functional Materials 2012, 22(7), 1385-1390.

[16] Yan, Y.; Ge, X.; Liu, Z.; Wang, J.-Y.; Lee, J.-M.; Wang, X. Facile synthesis of low crystalline $\mathrm{MoS}_{2}$ nanosheet-coated CNTs for enhanced hydrogen evolution reaction. Nanoscale 2013, 5(17), 7768-7771.

[17] Vrubel, H.; Merki, D.; Hu, X. Hydrogen evolution catalyzed by $\mathrm{MoS}_{3}$ and $\mathrm{MoS}_{2}$ particles. Energy \& Environmental Science 2012, 5(3), 6136-6144.

[18] Li, H.; Tsai, C.; Koh, A. L.; Cai, L. L.; Contryman, A. W.; Fragapane, A. H.; Zhao, J. H.; Han, H. S.; Manoharan, H. C.; Abild-Pedersen, F. Activating and optimizing $\mathrm{MoS}_{2}$ basal planes for hydrogen evolution through the formation of strained sulphur vacancies. Nature Materials 2016, 15(1), 48-53. 
[19] Tsai, C.; Chan, K. R.; Norskov, J. K.; Abild-Pedersen, F. Theoretical insights into the hydrogen evolution activity of layered transition metal dichalcogenides. Surface Science 2015, 640, 133-140. 


\section{Chapter 6}

Iron nanoparticles embedded in a graphitic carbon matrix as heterogeneous catalysts for the oxidative $\mathrm{C}-\mathrm{N}$ coupling of aromatic $\mathrm{N}-\mathrm{H}$ compounds and amides

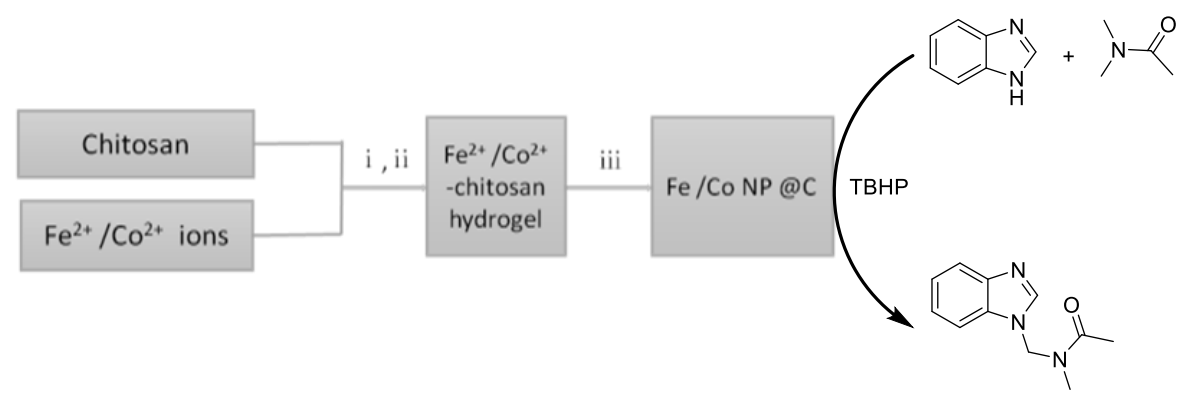





\subsection{Introduction}

As it has been commented in the Introduction, MNPs are catalysts for a wide range of organic reactions including oxidations, reductions, homo- and cross couplings, and rearrangements among other transformations. ${ }^{[1-4]}$ It has been already indicated that one general problem of MNPs that limits its application in catalysis is their low stability under the reaction conditions due to the tendency of the most active small MNPs to increase their particle size. ${ }^{[4]}$ Large particle size is generally associated to low or negligible catalytic activity. ${ }^{[2,5,6]}$

A general strategy to minimize particle growth is incorporation of MNPs in porous polymeric or inorganic matrices that by interaction with the particle or by confinement stabilizes their size. ${ }^{[7-10]}$ Besides the stabilization of particle-size distribution, the matrix can assist the catalytic reaction by adsorption or preactivation of the substrates. For these reasons, it is commonly observed that the nature and structure of the matrix exerts a strong influence on the intrinsic activity of the adsorbed or incorporated MNPs. ${ }^{[11-13]}$

In the introduction, we have already indicated that MNPs and metal oxides obtained by pyrolysis of metal complexes can exhibit a unique catalytic activity due to the favorable influence of the carbonaceous matrix surrounding the MNPs, which is formed in the process. ${ }^{[14,15]}$ In an analogous procedure, MNPs and metal oxide NPs wrapped with carbon have been obtained by pyrolysis of metal organic frameworks. ${ }^{[16-21]}$

Another aspect to be considered in the catalysis by transition MNPs is the nature of the metal. Although NPs of noble metals are widely used as catalysts, the interest in exploiting the potential of abundant, base transition metals as catalysts is increasing with regard to sustainability and affordability. ${ }^{[25,26]}$ In this context, Fe, the most abundant metal, has been under the spotlight because of its ability to promote organic reactions and couplings in particular. ${ }^{[26,27]}$

This Chapter describes a general procedure to prepare MNPs of Fe, Co and Fe-Co 
alloys embedded in a graphitic carbon matrix (denoted as MNP@C) derived from natural biopolymers. Although active carbons and other types of carbons are among the preferred supports of MNPs, ${ }^{[22-24]}$ the carbon-wrapped MNPs reported herein stand out by their pyrolytic formation procedure, the type of carbon that surrounds the MNPs, and the unique stability of MNPs that can be achieved.

The catalytic activity of these MNP@C samples has been determined for the oxidative $\mathrm{C}-\mathrm{N}$ cross coupling of aromatic $\mathrm{N}-\mathrm{H}$ compounds and amides, gaining some information on the operating reaction mechanism involving radicals. Related precedents have reported the oxidative $\mathrm{C}-\mathrm{N}$ coupling of aromatic $\mathrm{N}-\mathrm{H}$ compounds with amides using di-tert-butyl peroxide (DTBP) or tertiary-butyl hydro peroxide (TBHP) as oxidizing agent and homogeneous $\mathrm{Fe}^{2+}$ catalysts ${ }^{[28,29]}$ as well as a $\mathrm{Cu}$ metal organic framework as heterogeneous catalyst. ${ }^{[30]}$ However, MNPs wrapped in graphitic carbon, which are currently under intense investigation because of their unique catalytic activity that has frequently been observed, ${ }^{[31-34]}$ have not yet been reported as catalysts for this reaction.

\subsection{Results and discussion}

\subsubsection{Catalyst preparation and characterization}

Preparation of MNP@C was performed by pyrolyzing at $900{ }^{\circ} \mathrm{C}$ under inert atmosphere powdered samples of chitosan-containing adsorbed $\mathrm{Fe}^{2+}$ or $\mathrm{Co}^{2+}$ metal ions or the corresponding $\mathrm{Fe}^{2+}-\mathrm{Co}^{2+}$ mixture. The chitosan samples containing $\mathrm{Fe}^{2+} / \mathrm{Co}^{2+}$ metal ions were obtained by water evaporation of acid aqueous solutions of chitosan to which the appropriate amounts of the corresponding transition-metal ions (5 wt\% of metal ion with respect to dry chitosan weight) were added. The 
preparation procedure is illustrated in Scheme 6.1. As it has been presented in the introduction, our group has shown in a series of prior studies that pyrolysis of chitosan under inert atmosphere produces a $\mathrm{N}$-doped turbostratic graphitic carbon residue with some residual oxygen content (about 8 wt\%) that can be easily exfoliated by sonication. ${ }^{[35,36]}$ If these samples contain metals, they can be reduced to their metallic state by the so-called carbochemical reduction, while spontaneously undergoing simultaneous segregation in a different phase. ${ }^{[37]}$ Upon heating a mixture of metal oxide and carbon at high temperature, the oxide becomes reduced to metal and the carbon becomes oxidized to $\mathrm{CO}_{2} \cdot{ }^{[38]}$ The same behavior was expected to occur in the pyrolysis of $\mathrm{Fe}^{2+} / \mathrm{Co}^{2+}$-containing chitosan samples, resulting in the formation of MNP@C. Herein, a series of four MNP@C samples were prepared, and their elemental compositions according to chemical analysis are presented in Table 6.1. The " $x$ " in Fe-CoxNP@C denotes the Fe/Co atomic ratio.

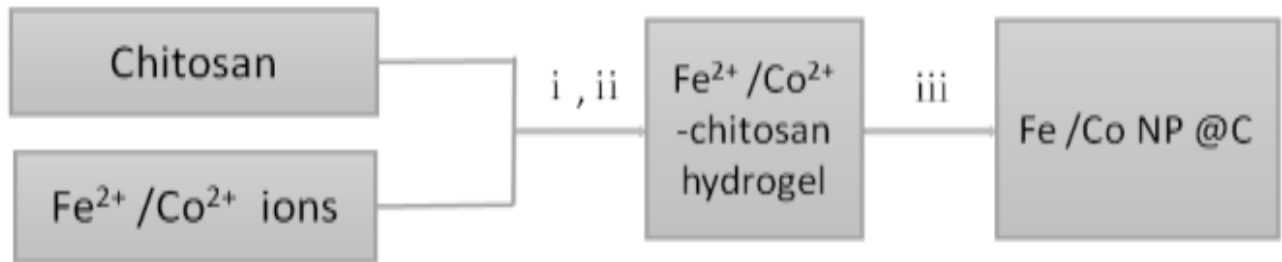

Scheme 6.1. Steps in the preparation of the MNP@C samples: (i) dissolution in acetic acid aqueous solutions; (ii) water evaporation and sample drying; (iii) pyrolysis at $900{ }^{\circ} \mathrm{C}$ under Ar flow. 
Table 6.1. Chemical analysis of the MNP@C samples. The percentage of metals was determined by ICP analysis and the percentage of $\mathrm{N}, \mathrm{C}, \mathrm{H}$ was determined by combustion analysis (experimental conditions are provided in Chapter 8). All values are in wt\%, and it is assumed that the difference to $100 \%$ is due to 0 content.

\begin{tabular}{llllll}
\hline Sample & $\mathrm{N}$ & $\mathrm{C}$ & $\mathrm{H}$ & $\mathrm{Fe}$ & $\mathrm{Co}$ \\
\hline FeNP@C & 0.98 & 89.76 & 0.14 & 1.02 & - \\
Fe-Co $1.42 \mathrm{NP} @ \mathrm{C}$ & 0.97 & 85.23 & 0.16 & 0.37 & 0.26 \\
Fe-Co $0.86 \mathrm{NP@C}$ & 1.04 & 86.02 & 0.16 & 0.62 & 0.72 \\
CoNP@C & 1.36 & 87.50 & 0.17 & - & 0.61 \\
\hline
\end{tabular}

Besides chemical analysis, the samples obtained according to Scheme 6.1 were characterized by their XRD patterns, Raman spectroscopy, and electron microscopy. XRD patterns of three selected MNP@C samples are shown in Figure 6.1. From the XRD patterns, the presence of a significant proportion of the corresponding metal carbides or metal oxides was ruled out, because the expected diffraction peaks for these metal carbides were either absent or they had very low intensity. Particularly for FeNP@C, weak diffraction peaks at $2 \theta 37^{\circ}(112)$ and $39^{\circ}(200)$ were recorded that are compatible with the presence of cementite $\left(\mathrm{Fe}_{3} \mathrm{C}\right),{ }^{[39]}$ but their intensity was very low. In contrast, those peaks indicating the presence of Fe or Co metals were in all cases very intense. For the mixtures of $\mathrm{Fe}$ and $\mathrm{Co}$, the minor variation in the position of the diffraction peaks at about $2 \theta 44^{\circ}$ supports the assumption that the NPs are real Fe-Co alloys, rather than independent Fe and CoNPs. By using the Scherrer equation, the average sizes of MNPs of the four samples were estimated to be 26.5,

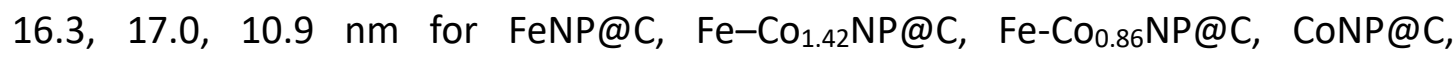
respectively, which agree well with the statistical average particle sizes obtained from TEM measurements. It should be commented that although no changes were 
observed in the XRD patterns, it is very likely that, after sample preparation and storage of the solids in vials at the ambient atmosphere, the outermost external part of the metal surface can become partially oxidized.

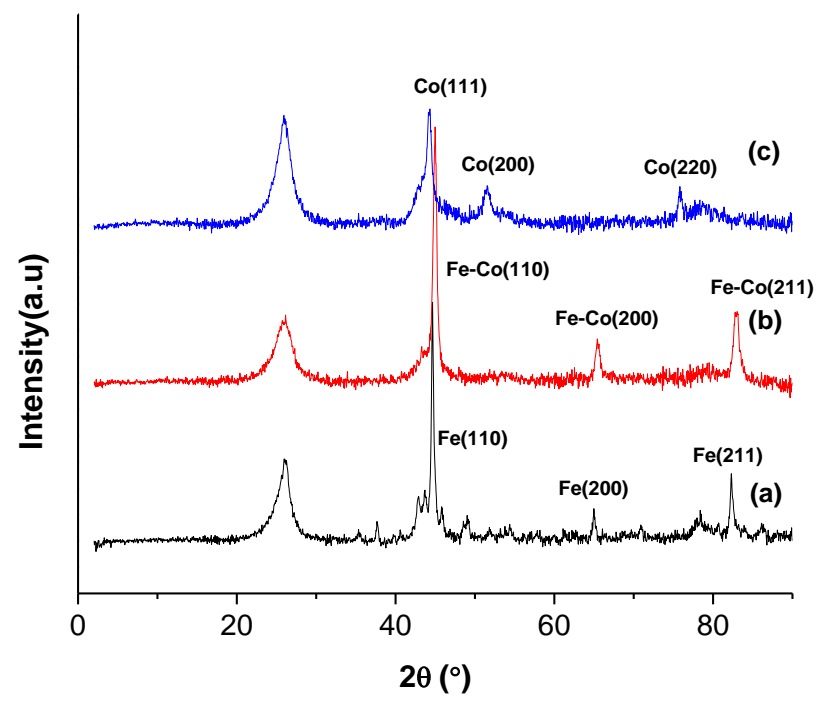

Figure 6.1. XRD patterns of the MNP@C samples prepared according to Scheme 6.1: (a) FeNP@C; (b) Fe-Co ${ }_{1.42}$ NP@C; (c) CoNP@C. The broad peak at $2 \theta 27^{\circ}$ corresponds to the graphitic carbon residue and some weak peaks in plot (a) could indicate the presence of some $\mathrm{Fe}_{3} \mathrm{C}$ (see text).

The nature of carbon residue derived from chitosan was determined by Raman spectroscopy. The Raman spectrum for FeNP@C is shown in Figure 6.2a. Very similar Raman spectra to those previously reported for turbostratic graphitic carbons lacking any metal were recorded for all the samples. ${ }^{[36]}$ Thus, the expected $G$ and $D$ bands appearing at 1596 and $1354 \mathrm{~cm}^{-1}$, respectively, were recorded together with two 2D defined peaks at 2940 and $2686 \mathrm{~cm}^{-1}$. This is in accordance with the presence in the graphene sheet of defects consisting in carbon vacancies, holes, and the residual $\mathrm{N}$ atoms from the chitosan precursor. ${ }^{[36]} \mathrm{A}$ quantitative indicator of the defect level of graphenic sheets can be given by determining the relative intensity of $G$ versus $D$ band $\left(I_{G} / I_{D}\right)$, which, in the present case, was 1.26 for the four MNP@C samples, 
corresponding to a defective, doped G. Interestingly, no vibration peaks attributable to the presence of metal oxides that should appear at low wavenumber values were observed for any of the MNP@C samples before their use as catalyst. This lack of metal oxide detection in Raman spectroscopy is in good agreement with the XRD patterns in which no oxides are detectable. In contrast, the recovered FeNP@C catalyst after the $\mathrm{C}-\mathrm{N}$ coupling between benzimidazole (BIM) and $\mathrm{N}, \mathrm{N}$-dimethylacetamide (DMA) showed the presence of iron oxide indicating that the oxidation by TBHP takes place under the experimental conditions (Figure 6.2b).

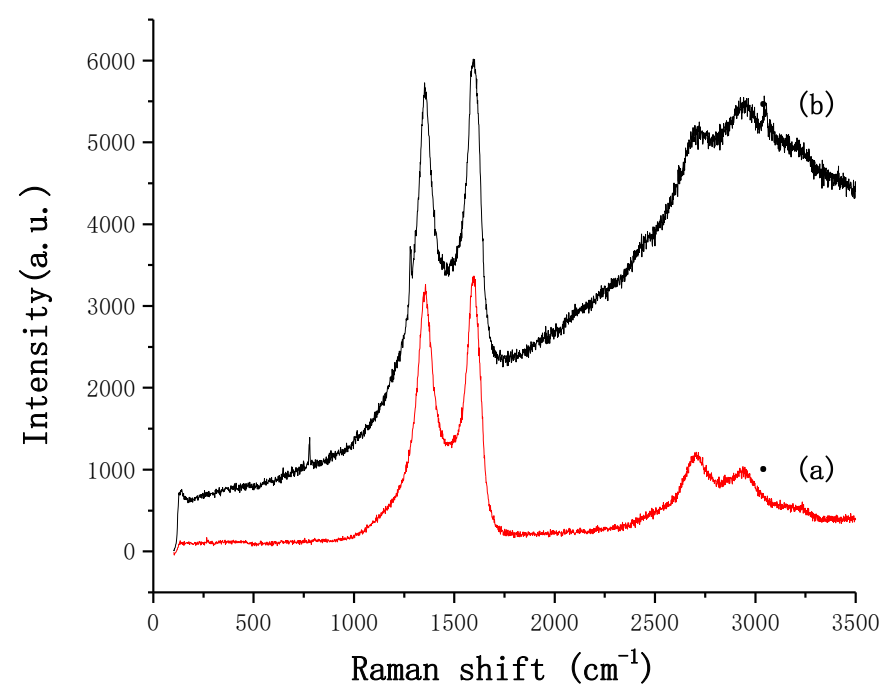

Figure 6.2. Raman spectra of fresh (a) and recovered (b) FeNP@C catalyst after the C-N coupling reaction.

To prove the accessibility to Fe and Co NPs even if they are embedded within the graphitic carbon matrix, the FeNP@C and CoNP@C samples were treated with an aqueous solution of diluted $\mathrm{HCl}$. To check the accessibility to the MNPs, portions of the as-synthesized samples (FeNP@C, Fe- $\mathrm{Co}_{0.86} \mathrm{NP} @ \mathrm{C}$ or CoNP@C, respectively) were treated in $0.5 \mathrm{M} \mathrm{HCl}$ at room temperature under stirring for sufficiently long time. Then, the samples were filtrated and the amounts of leached metals were determined by ICP-OES. Relative to the total metal content, $87.3 \%$ of total iron 
content in FeNP@C and 85.5 \% of total iron content and $87.5 \%$ of total cobalt content in Fe-CoNP $0.86 @ \mathrm{C}$, and 88.4 \% of total cobalt content in CoNP@C were dissolved under these conditions. It is assumed that acid leaching under diluted $\mathrm{HCl}$ can remove the accessible Fe and Co NPs embedded in the graphite shells. From these results, it seems that the Fe and Co NPs in the as-synthesized catalysts have similar metal accessibility. This acid leaching test supports that the surfaces of Fe and Co metals should be accessible to interact with TBHP and reagents despite their embedment in a carbon matrix.

The presence of MNPs embedded in a carbonaceous matrix was confirmed by TEM images of the MNP@C samples. A set of images illustrating the structure and morphology of the FeNP@C samples are shown in Figure 6.3 while images of other samples are presented in the Figures 6.4-6.6. MNPs were clearly distinguished in dark field that also allows the estimation of the particle size distribution. From these images, it was clear that the MNP size was not homogeneous and that there was a broad dispersion of sizes below $50 \mathrm{~nm}$. However, the corresponding histogram of particle sizes counting a statistically relevant number of particles shows that particles of sizes between 5 and $10 \mathrm{~nm}$ are the most abundant in the MNP@C samples. 


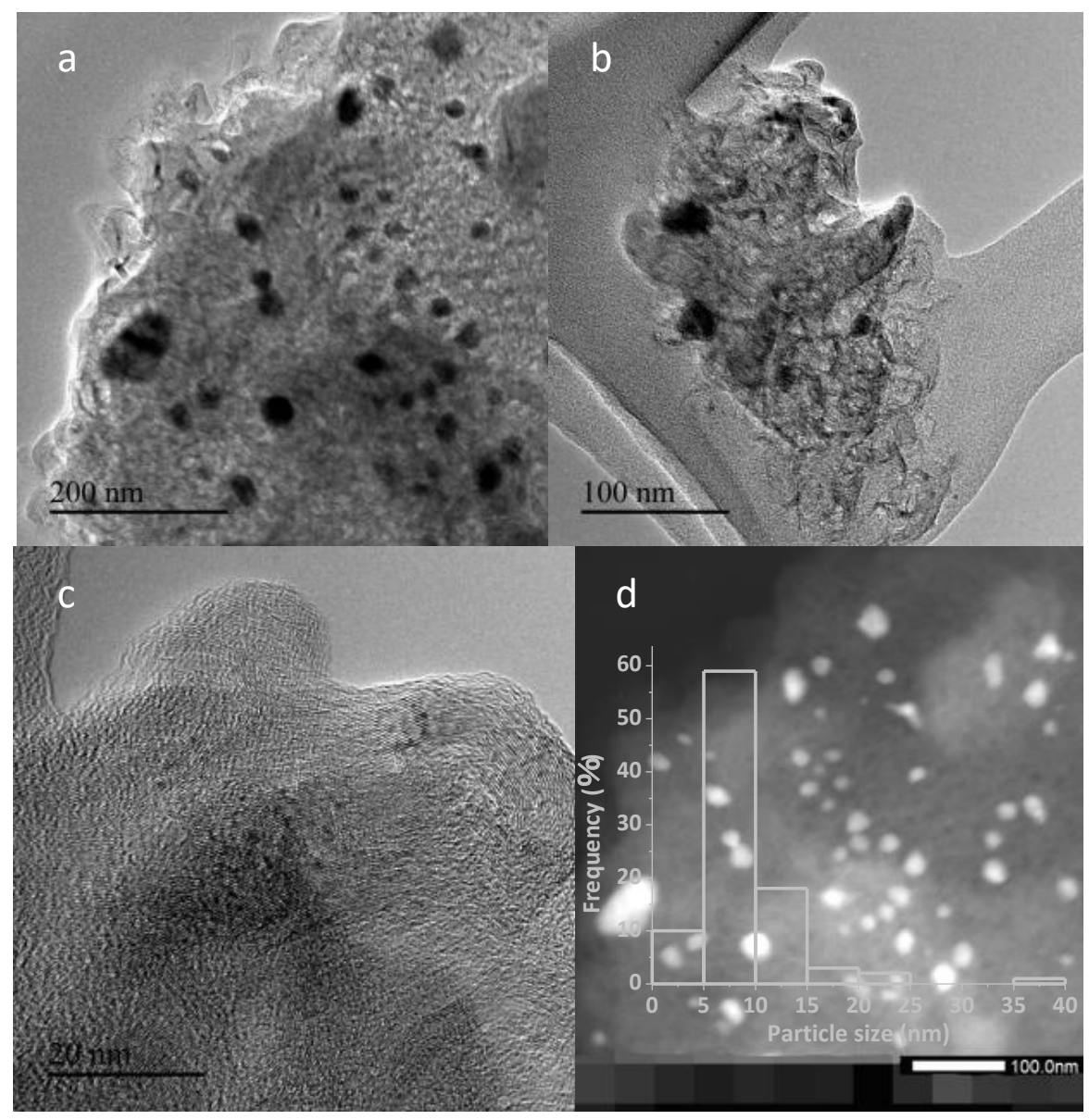

Figure 6.3. TEM images of FeNP@C samples at (a,b) low (scale bar 200 and 100 nm, respectively) and (c) high magnification (scale bar $20 \mathrm{~nm}$ ). (d) Dark field image, the inset shows the statistical particle size distribution of FeNPs (scale bar $100 \mathrm{~nm}$ ).

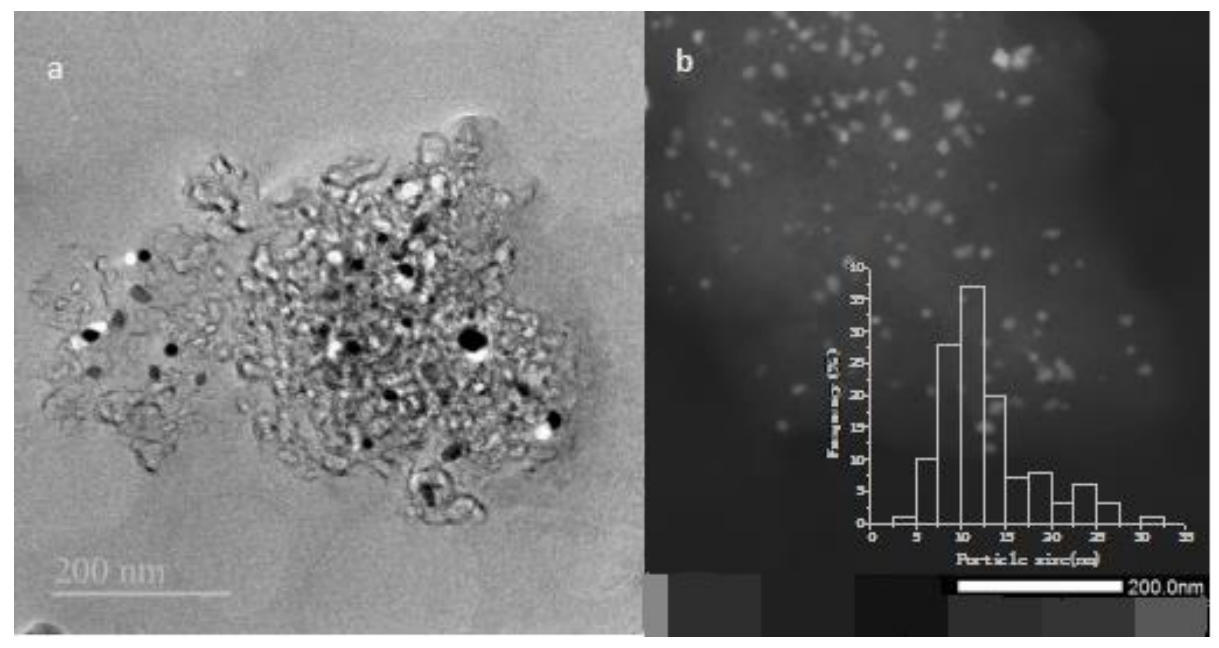

Figure 6.4. TEM images of Fe- $\mathrm{Co}_{1.42} \mathrm{NP} @ \mathrm{C}$ under bright (a) and dark (b) fields. The inset in image $\mathbf{b}$ shows the particle size distribution. 


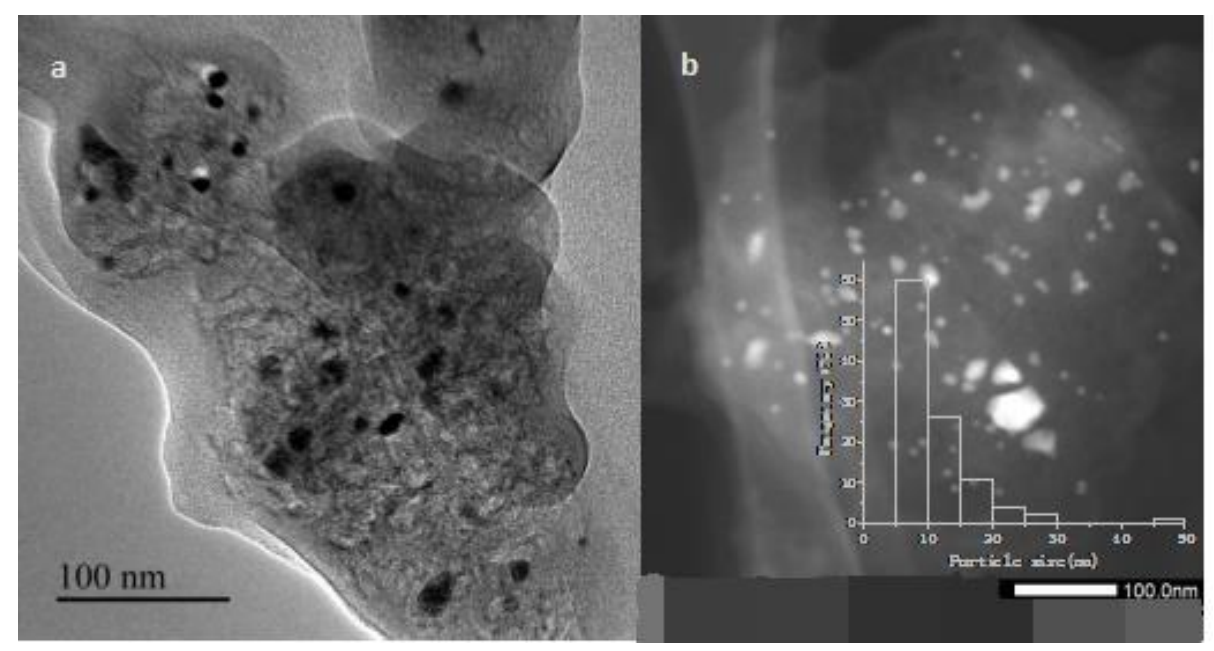

Figure 6.5. TEM images of Fe-Co ${ }_{0.86} \mathrm{NP} @ \mathrm{C}$ under bright (a) and dark (b) fields. The inset in image $\mathbf{b}$ shows the particle size distribution.

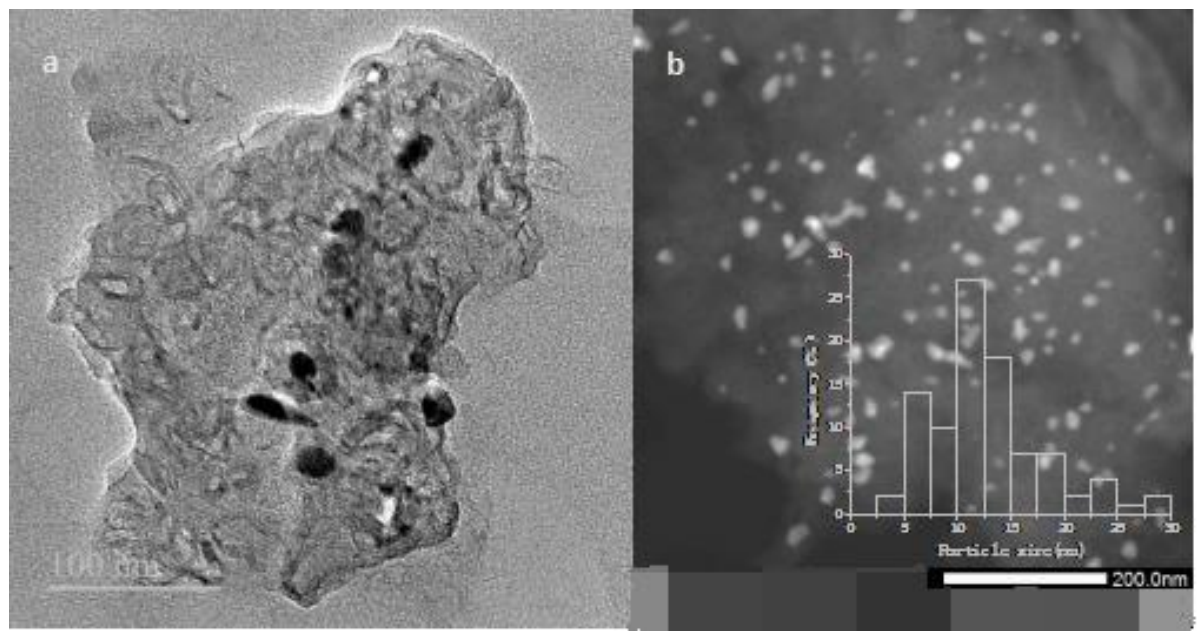

Figure 6.6. TEM images of CoNP@C under bright (a) and dark (b) fields. The inset in image $\mathbf{b}$ shows the particle size distribution.

EDX analysis of the location marked in the TEM image of Fe-Co $0.86 \mathrm{NP@C} \mathrm{shows}$ that the main metals in the sample are Fe and Co (see Figure 6.7). Furthermore, Elemental mapping of Fe and Co reveals that the locations of these two elements coincide (see Figure 6.8), thus, confirming the XRD results that the MNPs are real Fe-Co alloys. 


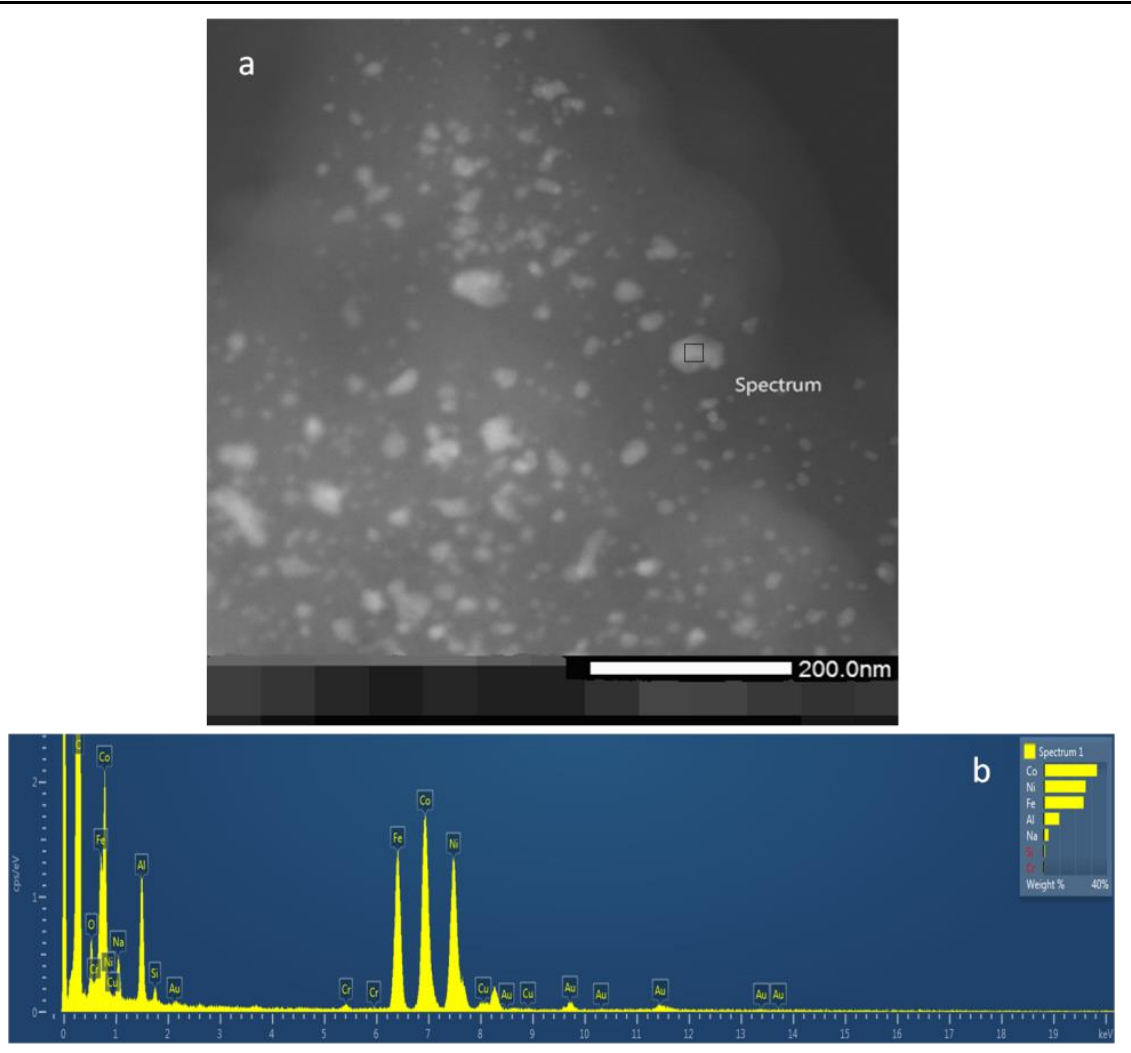

Figure 6.7. TEM image of Fe-Co ${ }_{0.86} \mathrm{NP} @ \mathrm{C}$ under dark field (a) and EDX analysis result of the marked location (b).

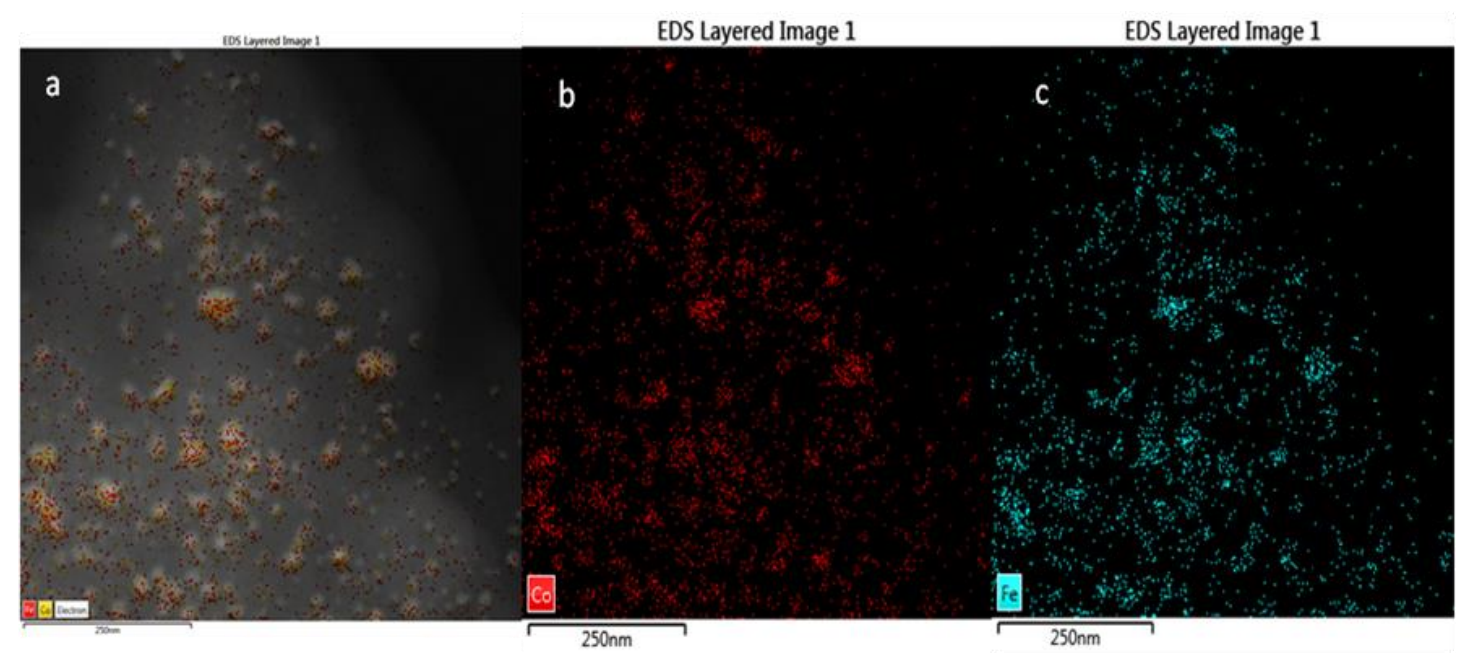

Figure 6.8. Images obtained from elemental mapping for Fe-Co $\mathrm{Co}_{0.86} \mathrm{NP} @ \mathrm{C}$ showing the elemental distribution of Fe and Co overlapped in one image (a) and in two separate images ( $b$ and $\mathbf{c}$ ). The elemental mapping images of Fe and Co are very similar, proving that the MNPs embedded in the graphitic matrix are Fe-Co alloys. 
The relatively small particle size measured for these MNPs is remarkable considering the high temperature $\left(900^{\circ} \mathrm{C}\right)$ to which the MNP@C samples are submitted during the preparation procedure and the time taken in the pyrolysis (6 h).

In one of the most common procedures to obtain FeNPs of small particle size similar to those obtained herein, these FeNPs were obtained by chemical reduction using reverse phase emulsions employing oleylamine-water emulsions. ${ }^{[40]}$ In this regard, although particles of much larger size are also detected, the present procedure allows obtaining in a single step Fe/CoNPs of small diameters and the carbon matrix in which the NPs are embedded. It is suggested that, as claimed previously, chitosan and the carbonaceous residues derived therefrom during the different phases of the pyrolysis are thwarting and restricting the growth of the Fe/CoNPs by interacting with them. ${ }^{[41]}$ In related precedents, it has been found that $\mathrm{Au}_{1}{ }^{[42,43]} \mathrm{Cu},{ }^{[41]}$ and even $\mathrm{TiO}_{2}{ }^{[44]}$ and $\mathrm{CeO}_{2}{ }^{[45]} \mathrm{NPs}$ of relatively small size are formed if the procedure starts with the corresponding metal ions embedded in chitosan.

The composition of the uppermost part of the FeNP@C catalyst was also characterized by XPS. The observed peaks as well as the best fitting to individual components are given in Figure 6.9. The deconvoluted C1s spectrum (Figure 6.9, left) can be fitted to three main peaks at 283.0, 284.5, and $288.5 \mathrm{eV}$ that can be assigned to $\mathrm{C}$ at defects, graphenic $\mathrm{C}$ atoms, and $\mathrm{sp}^{3} \mathrm{C}$ atoms bonded to oxygen, respectively. Furthermore, analysis of the Fe2p spectrum (Figure 6.9, right) reveals the existence of $\mathrm{Fe}^{2+}$ and $\mathrm{Fe}^{3+}$ at the binding energies of 709 and $711 \mathrm{eV}$, respectively. The presence of $\mathrm{Fe}^{2+\beta+}$ on the outermost part of FeNPs is relevant from a catalytic point of view and complements the information by XRD that indicates that the predominant oxidation state of $\mathrm{Fe}$ is $\mathrm{Fe}^{0}$. Notably, XPS analysis probes the composition of the outermost surface of the NPs, whereas XRD probes the whole sample. 

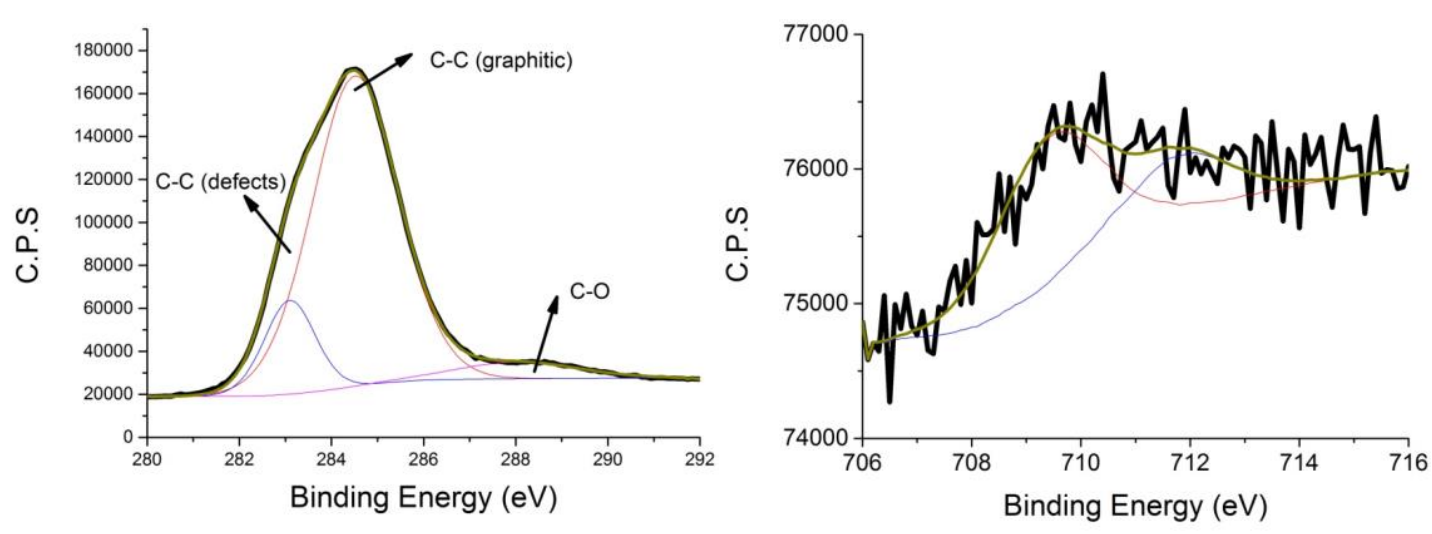

Figure 6.9. C 1s (left) and Fe $2 p$ (right) peaks of the high-resolution XPS of FeNP@C.

\subsubsection{Oxidative $\mathrm{C}-\mathrm{N}$ coupling}

The purpose of the present study was to evaluate the catalytic activity of the Fe/CoNP@C samples as heterogeneous catalysts for the oxidative C-N coupling. Oxidative $\mathrm{C}-\mathrm{N}$ couplings have the advantages that they do not require halide derivatives as substrates. Preliminary catalytic screenings were performed by using FeNP@C as catalyst, optimizing the amount of TBHP, the solvent, and the temperature for the coupling of benzimidazole (BIM) and N,N-Dimethylacetamide (DMA). The reaction is indicated in Scheme 6.2.

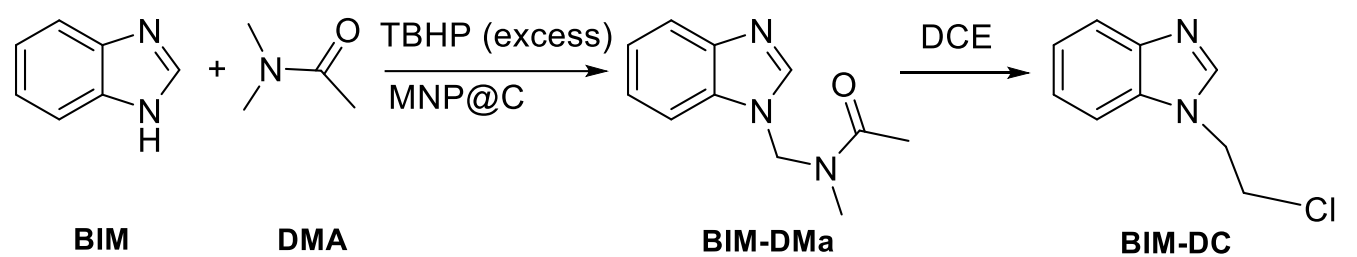

Scheme 6.2. Oxidative C-N coupling of benzimidazole (BIM) and N,N-dimethylacetamide (DMA) promoted by TBHP and FeNP@C.

Initially, 1,2-dichloroethane (DCE) was used as a solvent because literature data suggested that this could be one of the most suitable solvents for the reaction. ${ }^{[28]}$ However, in contrast to the report in the literature, ${ }^{[28]}$ under our reaction conditions 
the $\mathrm{C}-\mathrm{N}$ coupling product (BIM-DMa) was observed as a primary, but instable product, undergoing a subsequent conversion to $\mathrm{N}$-(2-chloroethyl)benzimidazole (BIM-DC) as final and stable product. A time-conversion plot of the catalytic oxidative $\mathrm{C}-\mathrm{N}$ coupling using DCE as a solvent is shown in Figure 6.10. The product BIM-DC clearly derives from the reaction of BIM with the solvent. Thus, to avoid the decomposition of BIM-DMa, the reaction was attempted in other solvents. It was observed, however, that the reaction completely failed using tert-butanol, toluene, acetonitrile, or ethyl acetate as solvents.

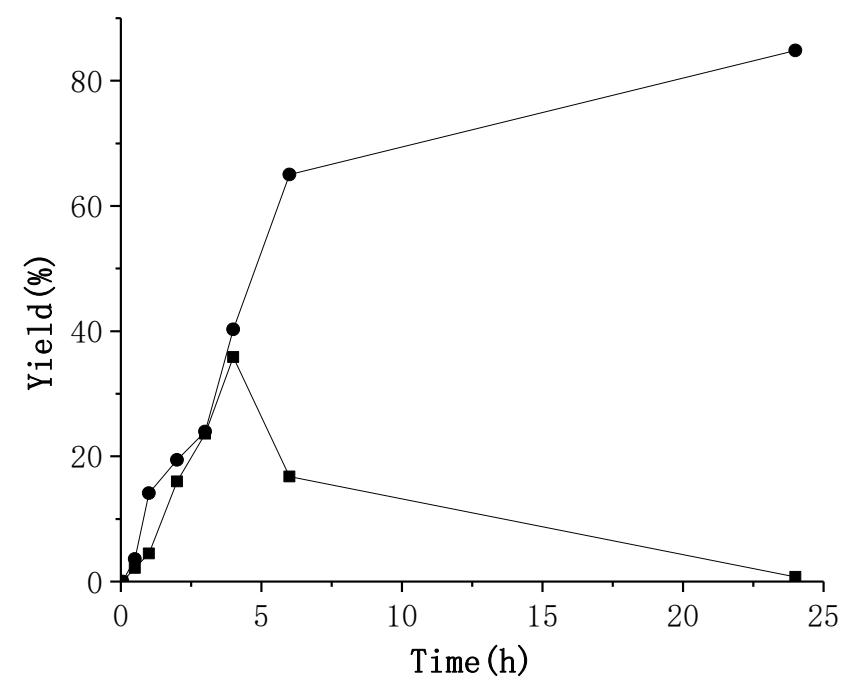

Figure 6.10. Time-yield plots determined by GC for the oxidative $\mathrm{C}-\mathrm{N}$ coupling promoted by FeNP@C in DCE solvent: (口) BIM-DMa; (•) BIM-DC. Reaction conditions: BIM (0.1 mmol), FeNP@C (1.6 mol\%), 2.5 equiv. oxidant (5 M solution of TBHP in decane), DCE (2 mL), Ar (2.5 bar), $110^{\circ} \mathrm{C}$.

In contrast to the negative results in some solvents, $\mathrm{C}-\mathrm{N}$ coupling between BIM and DMA could be also observed by using an excess of DMA that was acting under these conditions as reagent and solvent. In DMA, however, although the selectivity to BIM-DMa was almost complete, conversion of BIM was unsatisfactorily low, approximately $35 \%$. The temporal evolution of the formation of BIM-DMa under these conditions is shown in Figure 6.11. 
A reason for the low yield of BIM-DMa could be the spurious decomposition of TBHP without promoting $\mathrm{C}-\mathrm{N}$ coupling. This possibility was confirmed by performing an additional experiment in which the oxidative $\mathrm{C}-\mathrm{N}$ coupling reaction was initially started under the normal conditions, but as soon as the reaction rate decreased considerably, at approximately $4 \mathrm{~h}$ reaction time, 1.5 equivalents of TBHP were added (see Figure 6.11). Upon addition of the second amount of TBHP, a significant increase in the yield of BIM-DMa from approximately 30 to $50 \%$ was quickly achieved, reaching a final BIM-DMa yield of $65 \%$ at $20 \mathrm{~h}$. Interestingly, under the present experimental conditions, no other product besides BIM-DMa was observed and unreacted starting material BIM was also recovered at final reaction time. Thus, the incomplete yield probably results from the spurious decomposition of TBHP.

Considering the chemical structure of DMA and its reactivity through a N-methyl substituent, a structurally related solvent that could also be suitable to overcome the limitation of other solvents would be acetamide. Surprisingly, no BIM conversion was observed by using acetamide. Titration of TBHP after $24 \mathrm{~h}$ reaction time indicates that only a residual percentage of $11 \%$ of the initial TBHP remains, even though no BIM-DMa was formed. A reason for this could be that acetamide acts as a poison of FeNP@C catalyst by strong hydrogen bonding of acetamide to the metal surface. This possibility was checked by performing an experiment in which FeNP@C was boiled first in acetamide at $110{ }^{\circ} \mathrm{C}$ for $4 \mathrm{~h}$, then, it was recovered, washed with DMA, and used as catalyst in DMA as solvent. It was observed that the catalytic activity of acetamide-treated FeNP@C was much lower than that of the fresh untreated sample (see Figure 6.11). This decrease in catalytic activity after contacting with acetamide suggests that the material becomes deactivated by this solvent, thus, providing some hints to rationalize the negative results observed in acetamide, in spite of its similarity with DMA. 


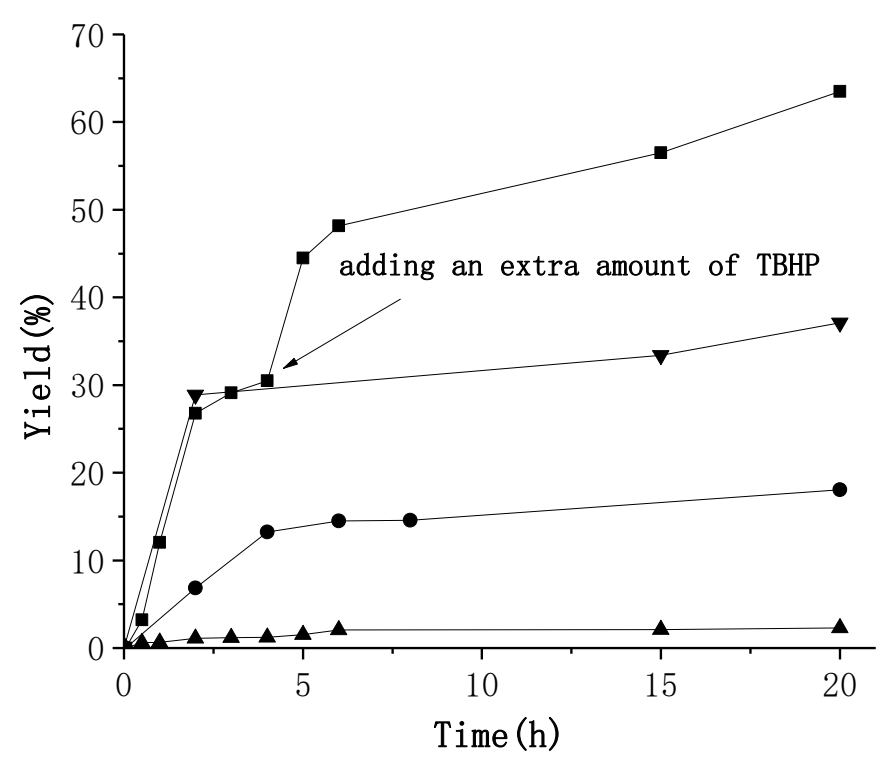

Figure 6.11. Time-yield plots determined by GC for two twin coupling reactions using the same reaction conditions; ( $\square$ ) adding an extra amount of TBHP (1.5 equiv. TBHP from a $5 \mathrm{M}$ solution of TBHP in decane) after $4 \mathrm{~h}$ reaction time; ( $\boldsymbol{\nabla})$ without addition; control experiment without any catalyst ( $\mathbf{\Delta}$ ) and with deactived catalyst obtained by boiling FeNP@C in acetamide before use (•). Reaction conditions: BIM (0.1 mmol), FeNP@C (1.6 mol\%), DMA (2 mL), Ar (2.5 bar), $110{ }^{\circ} \mathrm{C}, 2.5$ (or 4.0 ) equiv. of TBHP from a $5 \mathrm{M}$ TBHP solution in decane.

After having found that an excess of DMA is a suitable medium to perform the oxidative coupling and optimized reaction conditions, the influence on the catalytic performance of the percentage of Co on the Fe/CoNP@C catalyst was checked by performing a series of reactions under the same conditions, but using the other MNP@C catalysts of the series. As shown in Figure 6.12, the presence of Co in the catalyst is detrimental for the activity that undergoes a gradual decrease as the percentage of Co increases. 


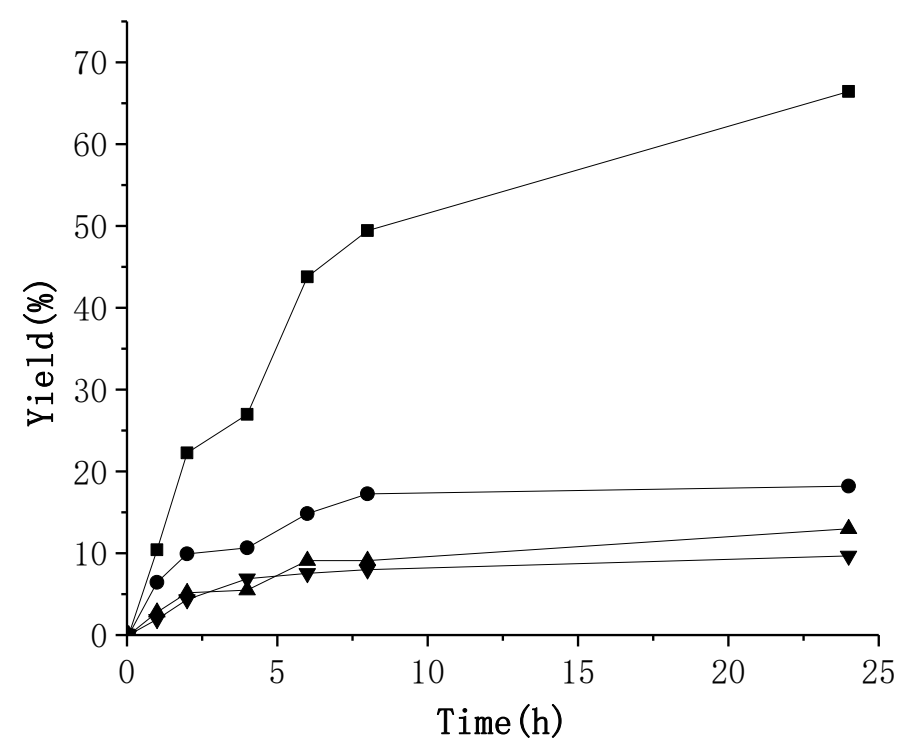

Figure 6.12. Time-yield plots determined by GC for the oxidative $\mathrm{C}-\mathrm{N}$ coupling of BIM and DMA in the presence of a series of catalysts: ( $\mathbf{\square})$ Fe P@C; $(\bullet)$ Fe-Co ${ }_{1.42} \mathrm{NP@C;} \mathrm{( \Delta )}$ Fe-Co $0.86 \mathrm{NP@C;}(\boldsymbol{\nabla})$ CoNP@C. Reaction conditions: BIM (0.1 mmol), catalyst (1.6 mol\%), two consecutive additions of 2.5 and 1.5 equiv. of TBHP (5 M solution in decane), DMA (2 mL), $\operatorname{Ar}\left(2.5\right.$ bar), $110^{\circ} \mathrm{C}$.

To gain insight into the heterogeneity of the MNP@C-catalyzed reaction, the solid catalysts were removed by filtration after $24 \mathrm{~h}$ reaction, and the clear solutions were analyzed by inductively coupled plasma optical emission spectroscopy (ICP-OES). Irrespective of the MNP@C material used, the Fe amount leached to the solution was $0.72 \mathrm{mg}$, whereas, for instance, the initial Fe amount of the FeNP@C (1.6 mol\%) with a Fe loading of $1.02 \mathrm{wt} \%$ was $93 \mathrm{mg}$. Thus, more than $99.2 \%$ of the Fe initially present was retained in the catalyst. It was, however, observed that filtration of the FeNP@C catalyst did not completely stop the reaction. Once initiated the reaction, formation of $\mathrm{C}-\mathrm{N}$ coupling products also occurred even after filtration of the FeNP@C catalyst at $4 \mathrm{~h}$, although in approximately $40 \%$ lower percentage than without filtration of the catalyst. This formation of product BIM-DMa in the absence of catalyst is proposed to be related to the reaction mechanism involving 
C-centered radicals as reaction intermediates and the role of FeNP@C as radical initiator as discussed below.

To learn more about the role of leached Fe species as a homogeneous catalyst, three control experiments using soluble precursors with $\mathrm{Fe}$ in different initial oxidation states were performed. Specifically, the compounds used were $\mathrm{Fe}(\mathrm{CO})_{5}$, $\mathrm{FeCl}_{2}$, and $\mathrm{Fe}(\mathrm{OAc})_{3}$, with $\mathrm{Fe}$ in $0,+2$, and +3 oxidation state, respectively. The amount of these materials used was such as to provide a weight of Fe approximately twice (1.5 $\mathrm{mg}$ ) of the leached Fe as determined by ICP at the final reaction time. It was, however, observed that although compound BIM-DMa was detected at final reaction time, the percentage of $\mathrm{C}-\mathrm{N}$ coupling was in all cases below $5 \%$, indicating that the contribution of the leached Fe to the observed yield of BIM-DMa should be negligible.

\subsubsection{Reusability and catalyst stability}

Reusability and catalyst stability are important issues in heterogeneous catalysis and have to be addressed. Aimed at determining catalyst stability, the same FeNP@C sample was submitted to a series of consecutive uses under the optimal reaction conditions. As shown in Figure 6.13, a gradual decrease in the catalytic activity was observed. Careful perusal of the time-conversion plots indicates, however, that the decay in the catalytic activity is mainly caused by the lower BIM-DMa yield achieved in the first TBHP addition (see data points at times shorter than $4 \mathrm{~h}$ in Figure 6.13), and the profile corresponding to the second addition is very similar with no decay in the reaction rates for five reuses. Thus, the decay observed in the yield at final reaction time seems to be owing to the operation of a very fast spurious decomposition of TBHP occurring for the used FeNP@C sample at the initial stages of the recycling and to some unavoidable losses of catalyst mass during reuse. It seems, however, that after this variation at initial times, the time-conversion plots are parallel upon reuse, indicating that the reaction rates for the oxidative $\mathrm{C}-\mathrm{N}$ coupling 
do not change upon reuse and that the activity of FeNP@C sample remains constant besides the spurious TBHP decomposition. It could be that this fast TBHP decomposition is an artifact caused by the recycling procedure.

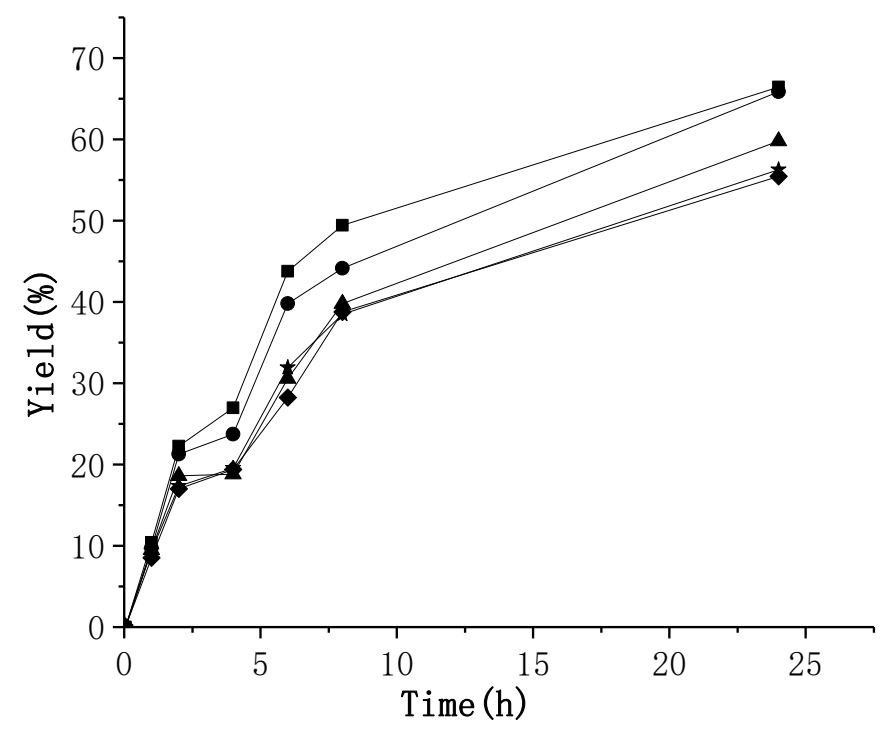

Figure 6.13. Time-yield plots determined by GC for the reusability test of the catalyst:

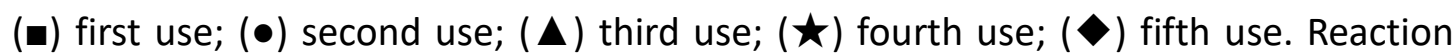
conditions: BIM (0.1 mmol), FeNP@C (1.6 mol\%), two consecutive additions of 2.5 and 1.5 equiv. of TBHP ( $5 \mathrm{M}$ solution in decane), DMA $(2 \mathrm{~mL}), \operatorname{Ar}(2.5$ bar $), 110^{\circ} \mathrm{C}$.

Furthermore, the FeNP@C sample resulting after five consecutive reuses was again imaged by TEM trying to determine variations of Fe particle size. It was, however, determined that the average Fe particle size did not undergo any clear variation upon use of the sample as catalyst. In addition, also the morphology of the C matrix remained unaltered. Images of one of the used samples are presented in Figure 6.14 , together with the corresponding particle size histogram. 


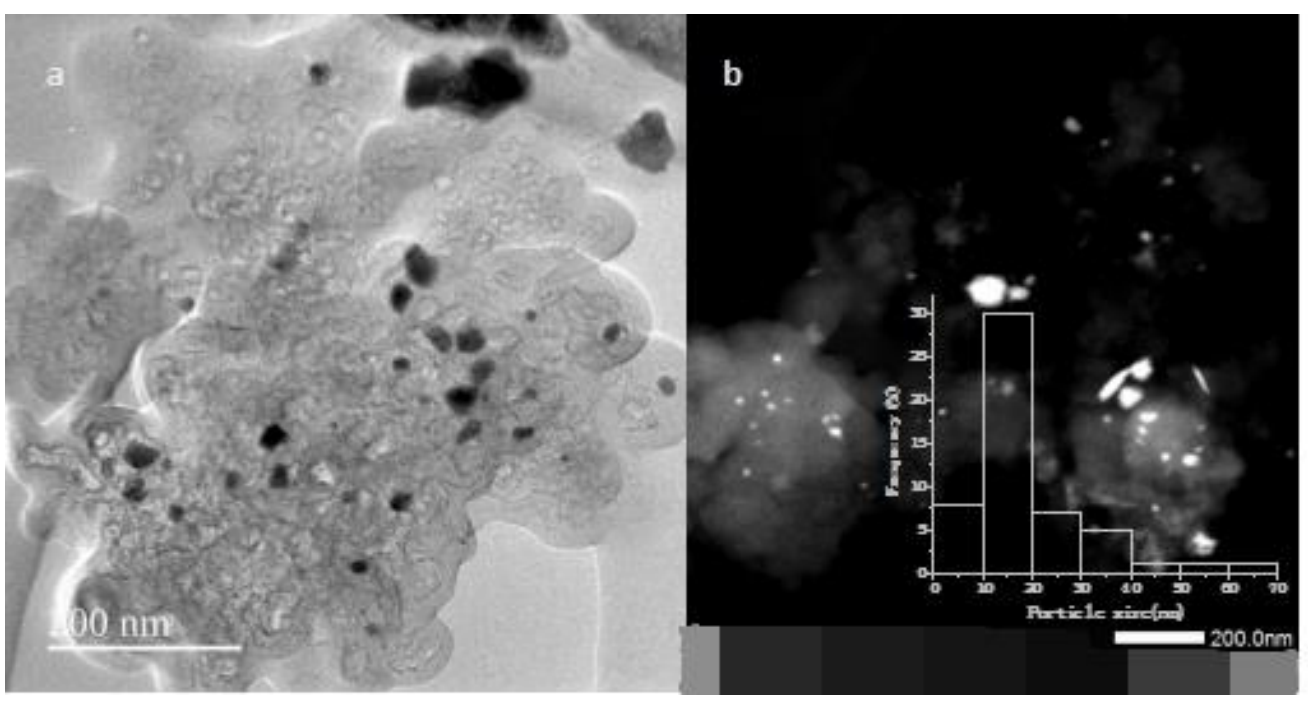

Figure 6.14. TEM images of five times-used FeNP@C under bright (a) and dark (b) field. The inset shows the particle size distribution of this used FeNP@C sample. Scale bars $200 \mathrm{~nm}$.

\subsubsection{Role of Cu impurities}

An additional point of concern using Fe as catalyst is the role of impurities on the observed catalytic activity. It should be noted that the samples in the present study were prepared by using a Fe salt precursor with a nominal purity of $99.99 \%$ and, therefore, the maximum expected proportion of metal impurities should be below $100 \mathrm{ppm}$. Considering the precedents that have shown that $\mathrm{Cu}$ is the most common Fe impurity that could contribute to the apparent catalytic activity of $\mathrm{Fe}$ in other cross-coupling reactions, ${ }^{[27,46]}$ the influence of the presence of $\mathrm{Cu}$ on the catalytic activity of FeNP@C was investigated by preparing two additional FeNP@C samples purposely containing 10 and $20 \mathrm{ppm}$ of $\mathrm{Cu}$. The results are presented in Figure 6.15. The presence of $\mathrm{Cu}$ even in minute amounts has a detrimental influence, decreasing the initial reaction rate of FeNP@C from 1.23 to $0.98 \mathrm{mmol} \mathrm{g}^{-1} \mathrm{~h}^{-1}$, and the yield at final time from 66.4 to $64.3 \%$. Extrapolation at 0 ppm of $\mathrm{Cu}$ content of the plot of the initial reaction rate against the level of $\mathrm{Cu}$ impurity strongly supports that Fe has intrinsic catalytic activity for the oxidative $\mathrm{C}-\mathrm{N}$ coupling. 


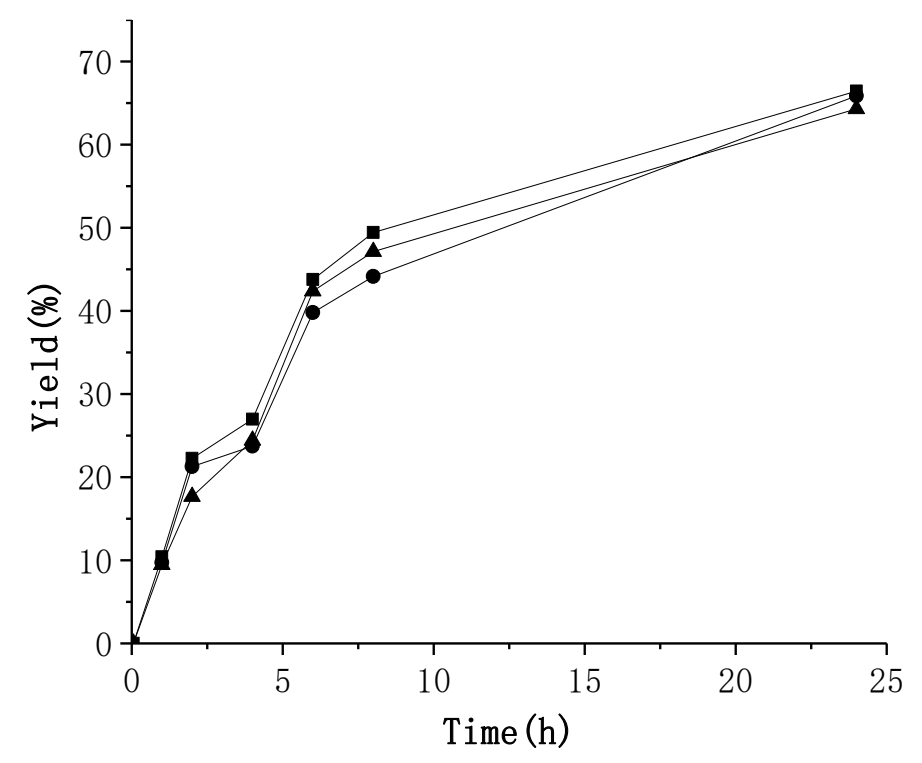

Figure 6.15. Time-yield plots determined by GC for the oxidative $\mathrm{C}-\mathrm{N}$ coupling with Fe salt precursor containing two different proportions of $\mathrm{Cu}$ impurities: (घ) Fe salt precursor with a purity of $99.99 \%$; ( $\mathbf{\Delta}$ ) Fe salt precursor with a purity of $99.99 \%$ contaminated with $10 \mathrm{ppm}$ of $\mathrm{Cu}$; (•) Fe salt precursor with a purity of $99.99 \%$ contaminated with 20 ppm of Cu. Reaction conditions: BIM (0.1 mmol), FeNP@C (1.6 mol\%), two consecutive additions of 2.5 and 1.5 equiv. of TBHP (5 M solution in decane), DMA (2 mL), $\operatorname{Ar}\left(2.5\right.$ bar), $110^{\circ} \mathrm{C}$.

\subsubsection{Reaction mechanism}

The reaction mechanism of the oxidative $\mathrm{C}-\mathrm{N}$ coupling, even in homogeneous phase, has not been yet disclosed. ${ }^{[28]}$ In order to gain some insights on the reaction mechanism, a series of control experiments were performed in the presence of (2,2,6,6-tetramethylpiperidin-1-yl)oxyl (TEMPO) as a quencher of possible C-centered radical intermediates. In this way, the reaction of DMA with BIM using FeNP@C as catalyst in the presence of TEMPO did not afford the expected product BIM-DMa (Scheme 6.3). In contrast, adducts between DMA with TEMPO (A) and the C-N coupling product with TEMPO (B) were observed from the analysis of the reaction mixture by GC-MS. On the other hand, a similar control experiment of DMA, TBHP and TEMPO in the absence of BIM resulted exclusively in the formation of adduct $\mathbf{A}$ 
between DMA and TEMPO. These experiments strongly support that the reaction proceeds through the formation of the radical from DMA.
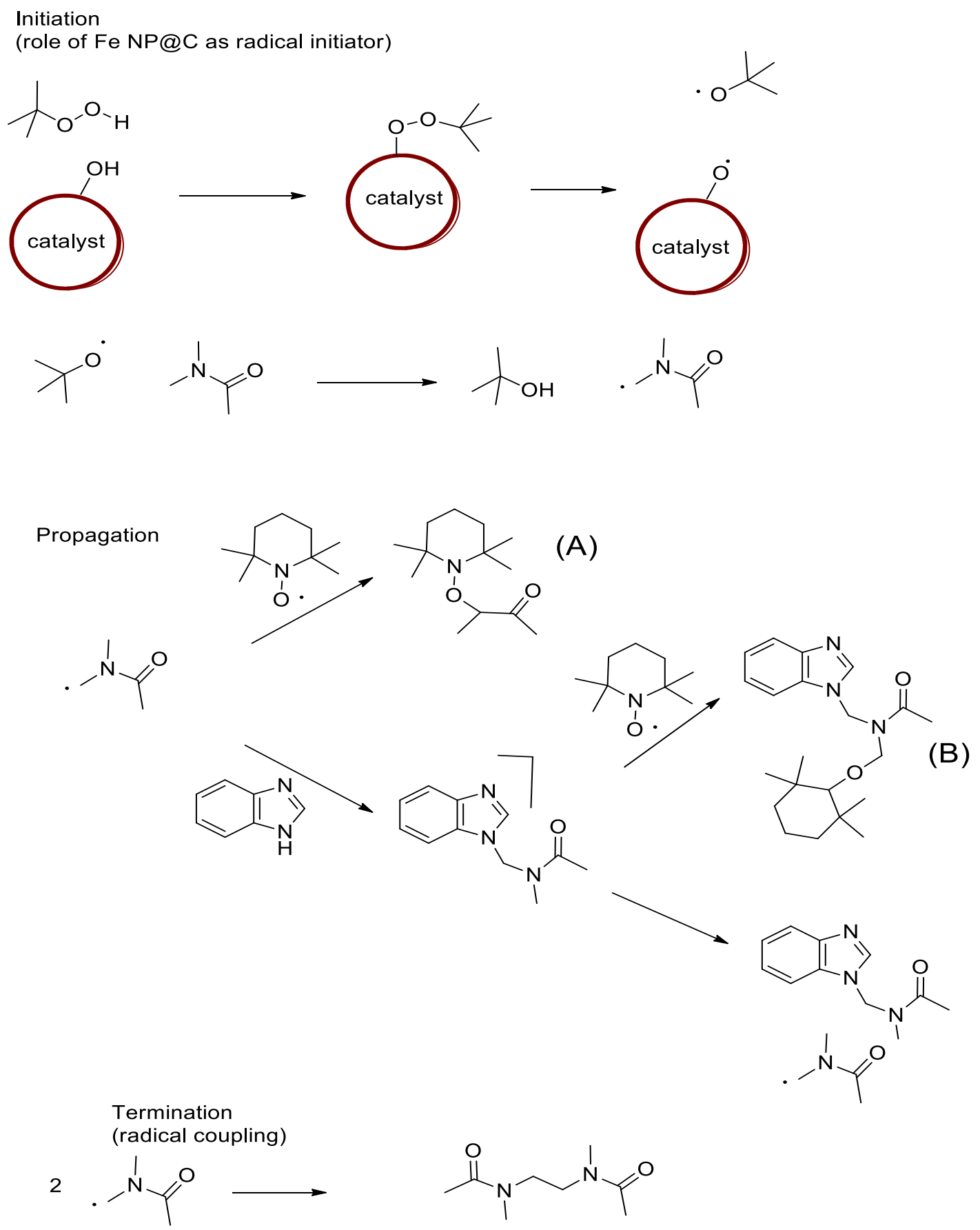

Scheme 6.3. Possible reaction mechanism, role of FeNP@C catalyst and quenching of intermediates by TEMPO.

Furthermore, if the adduct of DMA and TEMPO is first generated by TBHP in the 
presence of FeNP@C, and, then, the FeNP@C is removed, BIM is added and the mixture heated at $140^{\circ} \mathrm{C}$, formation of the $\mathrm{C}-\mathrm{N}$ coupling product BIM-DMa was again observed concomitantly with the disappearance of the DMA-TEMPO adduct A. This experiment is interpreted considering that the DMA-TEMPO adduct is acting as a dormant radical of DMA and upon heating, it would reversibly release some DMA and TEMPO radicals. This DMA radical would, then, couple with BIM without the need of any catalyst. In this way, the role of FeNP@C catalyst would be merely to act as initiator decomposing TBHP, generating the first radicals that would form C-centered DMA radicals.

As the homogeneous catalysts reported for this reaction are $\mathrm{Fe}(\mathrm{OAc})_{2}$ and $\mathrm{FeCl}_{2}$, and the XPS results reveal that the surface of FeNPs has a +2 and +3 oxidation state, it is probably that these surface species act similarly to the homogeneous counterparts coordinating to TBHP and, then, splitting the reagent in the initial oxygen-centered radicals (Scheme 6.3). This role of Fe generating radicals and the intermediacy of radicals is compatible with the observation indicated above that filtration of FeNP@C does not completely stop the reaction. As most radical reactions occur through radical chain mechanism, depending on the length of the propagation chain, some product formation can be observed even upon removal of the initiator, although the initiator can generate new radicals at any reaction time.

\subsubsection{Scope of the reaction}

Finally the scope of the reaction was screened by using FeNP@C as catalyst to promote the oxidative $\mathrm{C}-\mathrm{N}$ coupling of amides with other $\mathrm{N}-\mathrm{H}$ substrates. The results are presented in Table 6.2. As it can be seen there, high to moderate yields were obtained in all cases, except in the case of 2-phenylbenzimidazole, where low yields were attained. It seems that for 2-phenylbenzimidazole steric encumbrance due to the presence of a 2-phenyl substituent at the neighbor position should make $\mathrm{C}-\mathrm{N}$ coupling more difficult. In contrast, 2-methylbenzimidazole affords the expected C-N 
coupling product in even higher yields than the parent BIM. Similar increase in the yield of the coupling product is observed comparing the results obtained with pyrazole and 3,5-dimethylpyrazole. Besides using DMA, the scope of the oxidative C-N coupling was also expanded by using two other amides, namely, $\varepsilon$-caprolactam (CPL) and $\gamma$-butyrolactam (BUL), reaching good to moderate yields under the general reaction conditions.

Table 6.2. Products formed in the oxidative $\mathrm{C}-\mathrm{N}$ coupling of amides with $\mathrm{N}-\mathrm{H}$ substrates catalyzed by FeNP@C.[a]

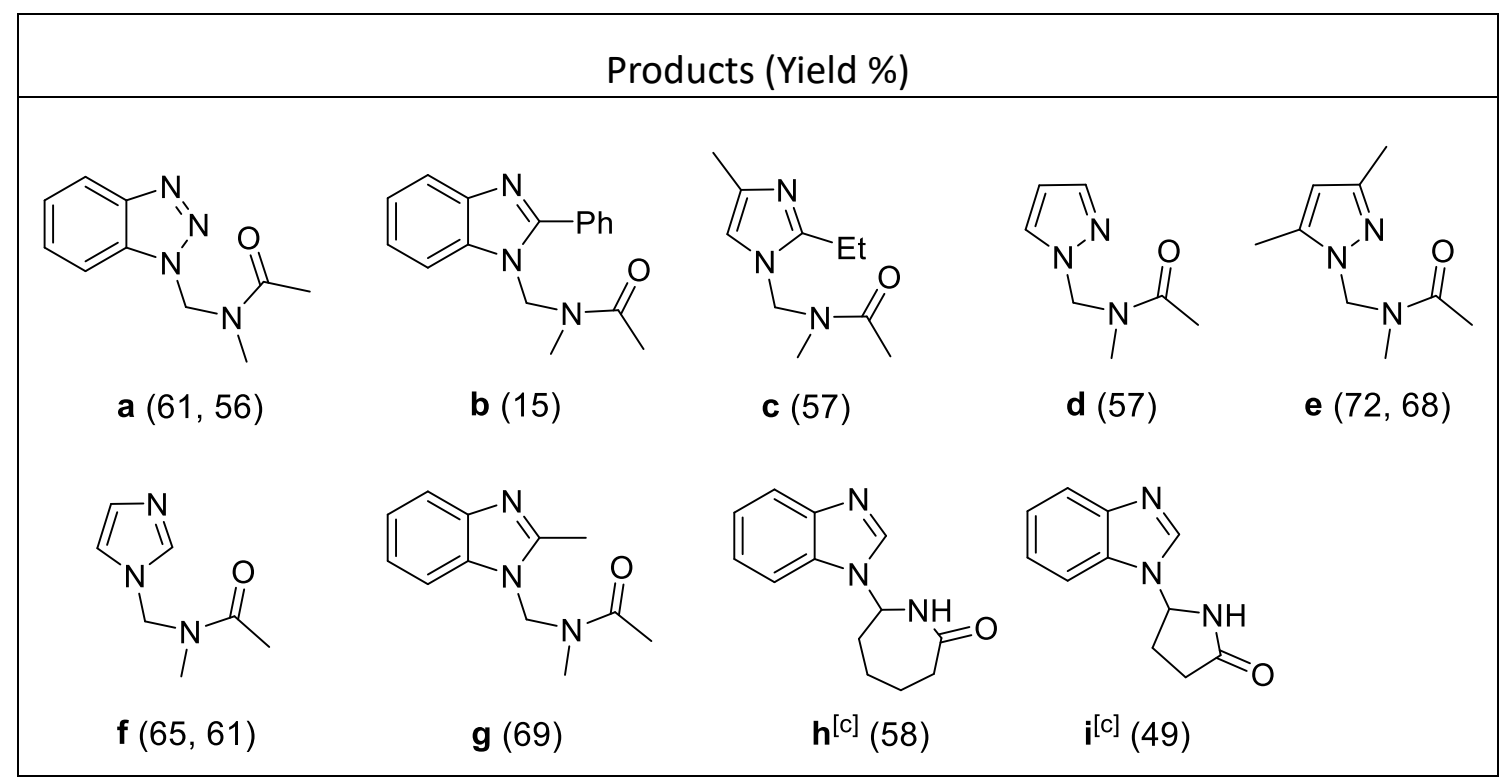

[a] Reaction conditions: $\mathrm{N}$ compound (0.1 mmol), FeNP@C (1.6 mol\%), two consecutive additions of 2.5 and 1.5 equiv. of TBHP (5 M solution in decane), amide (2 mL), $\operatorname{Ar}\left(2.5\right.$ bar), $110{ }^{\circ} \mathrm{C}, 24 \mathrm{~h}$; [b] the numbers in brackets correspond to the values determined by GC and if there is a second value, it corresponds to the isolated yield; [c] the reaction was performed with 5 mol\% of FeNP@C.

\subsection{Conclusions}

It is shown in this Chapter that chitosan-containing first-row transition metal ions, $\mathrm{Fe}^{2+}$ and $\mathrm{Co}^{2+}$, are suitable precursors to form MNPs, either of a single metal or 
as NP alloys, embedded within graphitic carbon matrix. The resulting carbonaceous MNP@C composites can be suitable materials to be used as heterogeneous catalysts. Their catalytic activity has been evaluated for the oxidative $\mathrm{C}-\mathrm{N}$ coupling, observing a large influence of the nature of the metal catalyst and the solvent in the reaction. In spite of the apparent decrease in catalytic activity, FeNP@C appears as a stable catalyst for the process with a broad scope. The reaction mechanism involves the generation of amide radicals originated from oxygen-centred radicals formed in the decomposition of TBHP promoted by the FeNP@C catalyst. 


\subsection{References}

[1] Astruc, D.; Lu, F.; Aranzaes, J. R. Nanoparticles as recyclable catalysts: the frontier between homogeneous and heterogeneous catalysis. Angewandte Chemie International Edition 2005, 44(48), 7852-7872.

[2] Haruta, M. Catalysis of gold nanoparticles deposited on metal oxides. Cattech 2002, 6(3), 102-115.

[3] Narayanan, R.; El-Sayed, M. A. Catalysis with transition metal nanoparticles in colloidal solution: nanoparticle shape dependence and stability. Journal of Physical Chemistry B 2005, 109, 12663-12676.

[4] Mikami, Y.; Dhakshinamoorthy, A.; Alvaro, M.; Garcia, H. Catalytic activity of unsupported gold nanoparticles. Catalysis Science \& Technology 2013, 3(1), 58-69.

[5] Haruta, M. Size-and support-dependency in the catalysis of gold. Catalysis Today 1997, 36(1), 153-166.

[6] Haruta, M. Gold as a novel catalyst in the 21st century: Preparation, working mechanism and applications. Gold Bulletin 2004, 37(1-2), 27-36.

[7] Chinchilla, R; Nájera, C. Recent advances in Sonogashira reactions. Chemical Society Reviews 2011, 40(10), 5084-5121.

[8] Dhakshinamoorthy, A.; Navalon, S.; Alvaro, M.; Garcia, H. Metal nanoparticles as heterogeneous Fenton catalysts. ChemSusChem 2012, 5(1), 46-64.

[9] Farina, V. High-turnover palladium catalysts in cross-coupling and Heck chemistry: A critical overview. Advanced Synthesis \& Catalysis 2004, 346(13-15), 1553-1582.

[10] White, R. J.; Luque, R.; Budarin, V. L.; Clark, J. H.; Macquarrie, D. J. Supported metal nanoparticles on porous materials. Methods and applications. Chemical Society Reviews 2009, 38(2), 481-494.

[11] Comotti, M.; Li, W. C.; Spliethoff, B.; Schuth, F. Support effect in high activity gold catalysts for CO oxidation. Journal of the American Chemical Society 2006, 128(3), 917-924.

[12] Lopez, N.; Janssens, T.; Clausen, B. S.; Xu, Y.; Mavrikakis, M.; Bligaard, T.; 
Nørskov, J. K. On the origin of the catalytic activity of gold nanoparticles for low-temperature CO oxidation. Journal of Catalysis 2004, 223(1), 232-235.

[13] Okumura, M.; Nakamura, S.; Tsubota, S.; Nakamura, T.; Azuma, M.; Haruta, M. Chemical vapor deposition of gold on $\mathrm{Al}_{2} \mathrm{O}_{3}, \mathrm{SiO}_{2}$, and $\mathrm{TiO}_{2}$ for the oxidation of $\mathrm{CO}$ and of $\mathrm{H}_{2}$. Catalysis Letters 1998, 51(1-2), 53-58.

[14] Pisiewicz, S.; Formenti, D.; Surkus, A.-E.; Pohl, M.-M.; Radnik, J.; Junge, K.; Topf, C.; Bachmann, S.; Scalone, M.; Beller, M. Synthesis of nickel nanoparticles with $\mathrm{N}$-doped graphene shells for catalytic reduction reactions. ChemCatChem 2016, 8(1), 129-134.

[15] Westerhaus, F. A.; Jagadeesh, R. V.; Wienhoefer, G.; Pohl, M.-M.; Radnik, J.; Surkus, A.-E.; Rabeah, J.; Junge, K.; Junge, H.; Nielsen, M.; Bruckner, A.; Beller, M. Heterogenized cobalt oxide catalysts for nitroarene reduction by pyrolysis of molecularly defined complexes. Nature Chemistry 2013, 5(6), 537-543.

[16] Banerjee, A.; Gokhale, R.; Bhatnagar, S.; Jog, J.; Bhardwaj, M.; Lefez, B.; Hannoyer, B.; Ogale, S. MOF derived porous carbon- $-\mathrm{Fe}_{3} \mathrm{O}_{4}$ nanocomposite as a high performance, recyclable environmental superadsorbent. Journal of Materials Chemistry 2012, 22(37), 19694-19699.

[17] Hu, J. A.; Wang, H. L.; Gao, Q. M.; Guo, H. L. Porous carbons prepared by using metal-organic framework as the precursor for supercapacitors. Carbon 2010, 48(12), 3599-3606.

[18] Wezendonk, T. A.; Warringa, Q.; Santos, V. P.; Chojecki, A.; Ruiten-beek, M.; Meima, G.; Makkee, M.; Kapteijn, F.; Gascon, J. Structural and elemental influence from various MOFs on the performance of Fe@C catalysts for Fischer-Tropsch synthesis. Faraday discussions, 2017, 197, 225-242.

[19] Wezendonk, T. A.; Santos, V. P.; Nasalevich, M. A.; Warringa, Q.; Dugulan, A. I.; Chojecki, A.; Koeken, A.; Ruitenbeek, M.; Meima, G.; Islam, H. U.; Sankar, G.; Makkee, M.; Kapteijn, F. Elucidating the nature of Fe species during pyrolysis of the Fe-BTC MOF into highly active and stable Fischer-Tropsch catalysts. J. ACS Catalysis 2016, 6(5), 3236-3247.

[20] An, B.; Cheng, K.; Wang, C.; Wang, Y.; Lin, W. Pyrolysis of metal-organic 
frameworks to $\mathrm{Fe}_{3} \mathrm{O}_{4} @ \mathrm{Fe}_{5} \mathrm{C}_{2}$ core-shell nanoparticles for Fischer-Tropsch synthesis. ACS Catalysis 2016, 6(6), 3610-3618.

[21] Santos, V. P.; Wezendonk, T. A.; Jaen, J.; Dugulan, A. I.; Nasale-vich, M. A.; Islam, H. U.; Chojecki, A.; Sartipi, S.; Sun, X.; Hakeem, A. A.; Kapteijn, F.; Makkee, M.; Gascon, J. Metal organic framework-mediated synthesis of highly active and stable Fischer-Tropsch catalysts. Nature Communications 2015, 6, 6451.

[22] De Jong, K. P.; Geus, J. W. Carbon nanofibers: catalytic synthesis and applications. Catalysis Reviews. Science and Engineering 2000, 42(4), 481-510.

[23] Joo, S. H.; Choi, S. J.; Oh, I.; Kwak, J.; Liu, Z.; Terasaki, O.; Ryoo, R. Ordered nanoporous arrays of carbon supporting high dispersions of platinum nanoparticles. Nature 2001, 412(6843), 169-172.

[24] Serp, P.; Corrias, M.; Kalck, P. Carbon nanotubes and nanofibers in catalysis. Applied Catalysis A 2003, 253(2), 337-358.

[25] Chen, Z.; Higgins, D.; Yu, A.; Zhang, L.; Zhang, J. A review on non-precious metal electrocatalysts for PEM fuel cells. Energy \& Environmental Science 2011, 4(9), 3167-3192.

[26] Sherry, B. D.; Fuerstner, A. The promise and challenge of iron-catalyzed cross coupling. Accounts of Chemical Research 2008, 41(11), 1500-1511.

[27] Thome, I.; Nijs, A.; Bolm, C. Trace metal impurities in catalysis. Chemical Society Reviews 2012, 41(3), 979-987.

[28] Saidulu, G.; Kumar, R. A.; Reddy, K. Iron-catalyzed C-N bond formation via oxidative $\mathrm{Csp}^{3} \mathrm{-H}$ bond functionalization adjacent to nitrogen in amides and anilines: Synthesis of N-alkyl and N-benzyl azoles. Tetrahedron Letters 2015, 56(28), 4200-4203.

[29] Xia, Q.; Chen, W. Iron-catalyzed N-alkylation of azoles via cleavage of an $\mathrm{sp}^{3}$ $\mathrm{C}-\mathrm{H}$ bond adjacent to a nitrogen atom. The Journal of Organic Chemistry 2012, 77(20), 9366-9373.

[30] Truong, T.; Nguyen, K. D.; Doan, S. H.; Phan, N. Efficient and recyclable $\mathrm{Cu}_{2}(\mathrm{BPDC})_{2}(\mathrm{DABCO})$-catalyzed direct amination of activated $\mathrm{sp}^{3} \mathrm{C}-\mathrm{H}$ bonds by $\mathrm{N}-\mathrm{H}$ heterocycles. Applied Catalysis A 2016, 510, 27-33. 
[31] Chen, F.; Topf, C.; Radnik, J.; Kreyenschulte, C.; Lund, H.; Schneider, M.; Surkus, A. E.; He, L.; Junge, K.; Beller, M. Stable and inert cobalt catalysts for highly selective and practical hydrogenation of $\mathrm{C}-\mathrm{N}$ and $\mathrm{C}-\mathrm{O}$ bonds. Journal of the American Chemical Society 2016, 138(28), 8781-8788.

[32] Cui, X. J.; Li, Y. H.; Bachmann, S.; Scalone, M.; Surkus, A. E.; Junge, K.; Topf, C.; Beller, M. Synthesis and characterization of iron-nitrogen-doped graphene/ core-shell catalysts: efficient oxidative dehydrogenation of $\mathrm{N}$-heterocycles. Journal of the American Chemical Society 2016, 138(33), 457.

[33] He, L.; Weniger, F.; Neumann, H.; Beller, M. Synthesis, characterization, and application of metal nanoparticles supported on nitrogen-doped carbon: catalysis beyond electrochemistry. Angewandte Chemie International Edition 2016, 55(41), $12582-12594$.

[34] Ziccarelli, I.; Neumann, H.; Kreyenschulte, C.; Gabriele, B.; Beller, M. Pd-supported on N-doped carbon: improved heterogeneous catalyst for base-free alkoxycarbonylation of aryl iodides. Chemical Communications 2016, 52(86), 12729-12732.

[35] Primo, A.; Atienzar, P.; Sanchez, E.; Delgado, J. M.; Garcia, H. From biomass wastes to large-area, high-quality, $\mathrm{N}$-doped graphene: catalyst-free carbonization of chitosan coatings on arbitrary substrates. Chemical Communications 2012, 48(74), 9254-9256.

[36] Primo, A.; Sanchez, E.; Delgado, J. M.; Garcia, H. High-yield production of $\mathrm{N}$-doped graphitic platelets by aqueous exfoliation of pyrolyzed chitosan. Carbon 2014, 68, 777-783.

[37] Abellan, G.; Latorre-Sanchez, M.; Fornes, V.; Ribera, A.; Garcia, H. Graphene as a carbon source effects the nanometallurgy of nickel in $\mathrm{Ni}, \mathrm{Mn}$ layered double hydroxide-graphene oxide composites. Chemical Communications 2012, 48(93), 11416-11418.

[38] Latorre-Sanchez, M.; Atienzar, P.; Abellan, G.; Puche, M.; Fornes, V.; Ribera, A.; Garcia, $\mathrm{H}$. The synthesis of a hybrid graphene-nickel/manganese mixed oxide and its performance in lithium-ion batteries. Carbon 2012, 50(2), 518-525. 
[39] Park, E.; Zhang, J. Q.; Thomson, S.; Ostrovski, O.; Howe, R. Characterization of phases formed in the iron carbide process by X-ray diffraction, Mossbauer, X-ray photoelectron spectroscopy, and Raman spectroscopy analyses. Metallurgical and Materials Transactions B 2001, 32(5), 839-845.

[40] Peng, S.; Wang, C.; Xie, J.; Sun, S. Synthesis and stabilization of monodisperse Fe nanoparticles. Journal of the American Chemical Society 2006, 128(33), 10676-10677.

[41] Primo, A.; Esteve-Adell, I.; Blandez, J. F.; Dhakshinamoorthy, A.; Alvaro, M.; Candu, N.; Coman, S. M.; Parvulescu, V. I.; Garcia, H. High catalytic activity of oriented 2.0.0 copper(I) oxide grown on graphene film. Nature Communications 2015, 6, 8561. [42] Primo, A.; Esteve-Adell, I.; Coman, S. N.; Candu, N.; Parvulescu, V. I.; Garcia, H. One-step pyrolysis preparation of 1.1 .1 oriented gold nanoplatelets supported on graphene and six orders of magnitude enhancement of the resulting catalytic activity. Angewandte Chemie-International Edition 2016, 55 (2), 607-612.

[43] Mateo, D.; Esteve-Adell, I.; Albero, J.; Sanchez Royo, J. F.; Primo, A.; Garcia, H. 111 oriented gold nanoplatelets on multilayer graphene as visible light photocatalyst for overall water splitting. Nature Communications 2016, 7, 11819.

[44] Buaki-Sogo, M.; Serra, M.; Primo, A.; Alvaro, M.; Garcia, H. Alginate as template in the preparation of active titania photocatalysts. ChemCatChem 2013, 5(2), 513-518.

[45] Lavorato, C.; Primo, A.; Molinari, R.; Garcia, H. Natural alginate as a graphene precursor and template in the synthesis of nanoparticulate ceria/graphene water oxidation photocatalysts. ACS Catalysis 2014, 4(2), 497-504.

[46] Buchwald, S. L.; Bolm, C. On the role of metal contaminants in catalyses with $\mathrm{FeCl}_{3}$. Angewandte Chemie International Edition 2009, 48(31), 5586-5587. 



\section{Chapter 7}

Extremely high selectivity towards isobutane in $\mathrm{CO}_{2}$ hydrogenation catalyzed by Fe-Co alloy nanoparticles embedded in graphitic carbon matrix

$$
\mathrm{CO}_{2}+\mathrm{H}_{2} \text { i-Butane }+\mathrm{H}_{2} \mathrm{O}
$$

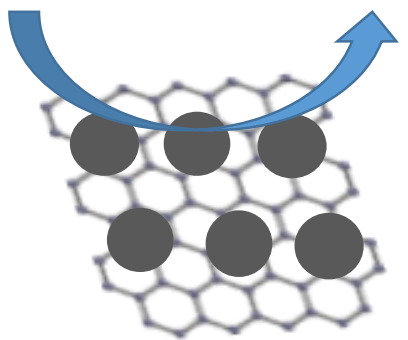

$\mathrm{Fe}_{\mathrm{x}} \mathrm{Co}_{\mathrm{y}} @(\mathrm{~N}) \mathrm{G}$ 



\subsection{Introduction}

The massive consumption of fossil fuels as primary energy source and, as a result, the corresponding atmospheric $\mathrm{CO}_{2}$ emissions, is considered the most important anthropogenic cause responsible for the global warming and climate change. ${ }^{[1]}$ For this reason, there is a strong international commitment to considerably decrease $\mathrm{CO}_{2}$ release into the atmosphere, ${ }^{[2-7]}$ there being a strong incentive for using $\mathrm{CO}_{2}$ as feedstock. The aim is the implementation of a circular economy in which $\mathrm{CO}_{2}$ is used in processes that can be carried out at commensurate scales as the current emission volumes. ${ }^{[8,9]}$

One of the main problems encountered in this strategy is the high stability of $\mathrm{CO}_{2}$ that makes most of the reactions involving this molecule thermodynamically uphill. ${ }^{[8,10-12]}$ Among the few exceptions to this general rule, one of the most appealing reactions is $\mathrm{CO}_{2}$ hydrogenation, since it renders hydrocarbons and other compounds that can be used as fuels, allowing $\mathrm{CO}_{2}$ recyclability. ${ }^{[9,13]} \mathrm{H}_{2}$ can become available in large amounts through water electrolysis using renewable electricity. ${ }^{[14]}$

The reaction of $\mathrm{CO}_{2}$ and $\mathrm{H}_{2}$ can be carried out at convenient rates and conversions at temperatures above $400^{\circ} \mathrm{C}$ to yield either $\mathrm{CO}$ (reverse water gas shift), $\mathrm{CH}_{4}$ (the Sabatier reaction), hydrocarbons (Fischer-Tropsch) or $\mathrm{CH}_{3} \mathrm{OH}$, among other possible products, depending on the catalyst and the reaction conditions. ${ }^{[8,12,13]}$ Due to their general activity in Fischer-Tropsch and water gas shift, materials based on supported Fe and Co nanoparticles (NPs), either in the metallic state or as their oxides, are among the most intensely studied heterogeneous catalysts for $\mathrm{CO}_{2}$ hydrogenation. ${ }^{[8,12,15,16]}$ However, it has been shown conclusively that Fe and Co NPs deposited on $\mathrm{Al}_{2} \mathrm{O}_{3}, \mathrm{SiO}_{2}$ and other supports render predominantly methane with a selectivity over $70 \%$, accompanied by much lesser amounts of mixtures of short-chain hydrocarbons. ${ }^{[13]}$ There is a remarkable difference in the hydrocarbon distribution in favor of methane when $\mathrm{CO}_{2}$ is the starting material compared to the Fischer-Tropsch process using $\mathrm{CO}$ as feedstock for the same or analogous Fe and Co 


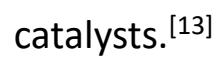

Regarding the reaction mechanism, it has been shown that Fe and Co oxides undergo partial or total reduction to the corresponding metal states and that during the initial stages of the reaction they form in some extent certain metal carbides that have been proposed in some cases as active sites for the reaction. ${ }^{[17,18]}$ For this reason, most probably, these Fe and Co NPs have also been supported on carbon materials, ${ }^{[19,20]}$ including carbon nanotubes. ${ }^{[21,22]}$ More recently, it has been reported that carbon-coated Fe and Co NPs, in which these metal NPs are surrounded by amorphous or graphitic carbon, can catalyze $\mathrm{CO}_{2}$ hydrogenation. ${ }^{[20]}$ However, the selective formation of isobutane has never been observed in any of these precedents.

In some of the reports about the preparation of these metal NPs wrapped by carbon, the procedure involves the pyrolysis of the appropriate metal complexes or crystalline metal organic frameworks. ${ }^{[23,24]}$ One possible disadvantage of these methods is the need of preparation of costly precursors following dedicated syntheses that afterwards are decomposed to a carbon residue. It would be more convenient to employ easy-to-make, available metal containing precursors that can be transformed into carbon surrounded metal NPs. ${ }^{[25,26]}$

In the previous Chapter we have reported that pyrolysis of iron chitosan hydrogel simply obtained by adding a $\mathrm{Fe}^{2+}$ salt to an aqueous solution of chitosan renders a Fe@C material that shows catalytic activity for the oxidative C-N coupling of amides and $\mathrm{N}-\mathrm{H}$ heterocycles. ${ }^{[27]}$

Considering that Fe and Co, as well as their alloys, are well-known catalysts for $\mathrm{CO}_{2}$ hydrogenation, ${ }^{[13,19,21,22]}$ it was of interest to check the catalytic activity of small metal NPs of these metals and their alloys surrounded by N-doped graphitic carbon prepared through a procedure that produces a strong binding between the NPs and graphitic matrix. ${ }^{[28,29]}$ Herein we present the outstanding isobutane selectivity, over $92 \%$ at over $87 \% \mathrm{CO}_{2}$ conversion, of Fe-Co alloy NPs strongly grafted on N-doped 
graphitic carbon. Isobutane is a significantly more valuable chemical than methane and the present findings open new avenues in the valorization of $\mathrm{CO}_{2}$, by obtaining selectively this $\mathrm{C}_{4}$ hydrocarbon. Furthermore, the catalyst is remarkably stable and does not undergo deactivation upon a prolonged time on stream (over $500 \mathrm{~h}$ ). Theoretical calculations suggest that this unprecedented selectivity arises from the unique metal-support interaction.

\subsection{Results and discussion}

Preparation of the samples under study is summarized in Scheme 7.1. The process starts with the mixing of an acidic aqueous solution of chitosan with another containing the required amounts of iron or cobalt acetates. Subsequently, the acid gel is precipitated as millimetric beads by dropwise addition with a syringe into an aqueous $\mathrm{NaOH}$ solution. A well-known property of chitosan is its ability to adsorb metal salts from water due to strong Coulombic interactions and hydrogen bridges with the aquated metal cations. ${ }^{[30,31]}$
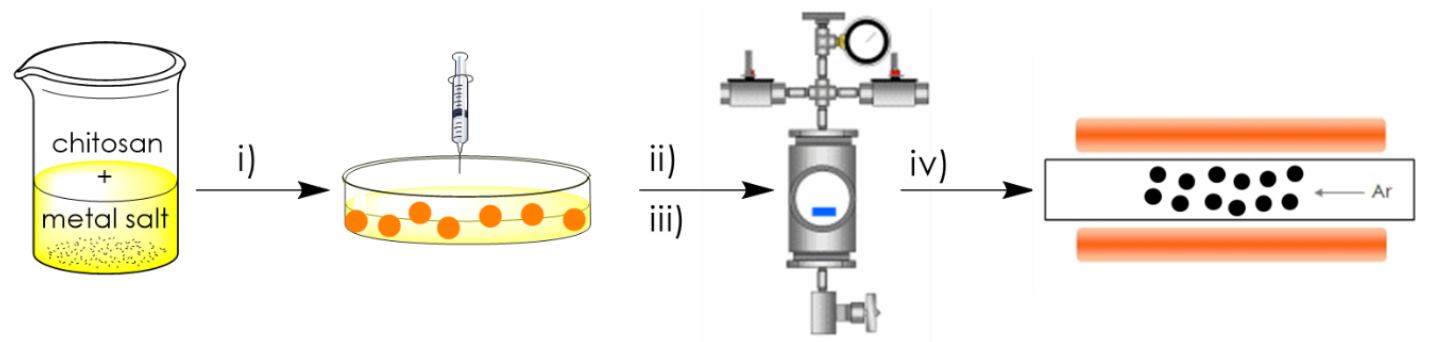

Scheme 7.1. Illustration of the preparation procedure of Fe/Co alloy NPs wrapped on $\mathrm{N}$-doped graphitic carbon matrix. i) Precipitation of chitosan beads by neutralization of acidic chitosan-metal aqueous solution. ii) Drying of the hydrogel by gradual exchange with ethanol. iii) Supercritical $\mathrm{CO}_{2}$ drying and formation of chitosan-metal aerogel. iv) Pyrolysis of the chitosan-metal aerogel. 
These hydrogel chitosan beads containing $\mathrm{Fe}^{3+}$ and $\mathrm{Co}^{2+}$ salts were dried by suspending them successively in five water-ethanol solutions having increasing ethanol percentage (90:10, 70:30, 50:50, 30:70 and 10:90 water:ethanol) and finally in pure ethanol. The resulting alcogel beads were submitted to supercritical $\mathrm{CO}_{2}$ drying to obtain highly porous aerogel beads of the metal-containing chitosan. The above-procedure has been well described in the literature to obtain high-surface area, porous chitosan beads. ${ }^{[32,33]}$ Other alternative drying procedures result in very low surface area chitosan samples as a consequence of the collapse of the porosity due to capillary forces and hydrogen bonding during the removal of water. ${ }^{[32,34]}$ After supercritical $\mathrm{CO}_{2}$ drying, the resulting chitosan aerogel beads were finally pyrolyzed at $900{ }^{\circ} \mathrm{C}$ to render the metal NPs surrounded by N-doped graphitic carbon. Four different samples (denoted as $\mathrm{Fe}_{x} \mathrm{Co}_{y} @(\mathrm{~N}) \mathrm{G}$ ) having Fe, Co or two alloys of Fe-Co with different atomic proportion were prepared.

The synthetic procedure for $\mathrm{Fe}_{x} \mathrm{Co}_{y} @(N) G$ in the Chapter was a little different from the procedure used for the similar materials in Chapter 6. In Chapter 6, Fe/Co NPs embedded in graphene were also prepared, but the sizes of the nanoparticles were quite large and the NPs were not well dispersed. To improve the quality of the samples, in the Chapter, aerogels of Fe/Co-chitosan were prepared by precipitating the metal-contained chitosan beads in a basic solution and then drying them in $\mathrm{CO}_{2}$ supercritical atmosphere before the samples were submitted for pyrolysis. By using this improved method, a composite material of graphene with more well dispersed smaller sized particles was obtained and showed higher catalytic activity.

The elemental composition of the Fe or Co containing graphitic samples after pyrolysis was determined by combustion chemical analysis to establish the carbon and nitrogen content and ICP/AOS analysis to quantify the content of each metal. Table 7.1 summarizes the main analytical and textural properties of the four samples prepared in the present study. 
Table 7.1. Analytical and textural data of the as-synthesised samples.

\begin{tabular}{|c|c|c|c|c|c|c|c|}
\hline Sample & $\begin{array}{c}C \\
(w t \%)\end{array}$ & $\begin{array}{c}N \\
(w t \%)\end{array}$ & $\begin{array}{c}\mathrm{Fe} \\
(w t \%)\end{array}$ & $\begin{array}{c}\text { Co } \\
\text { (wt\%) }\end{array}$ & $\begin{array}{c}\text { Total metal } \\
\text { (wt. \%) }\end{array}$ & $\begin{array}{c}\text { Particle } \\
\text { size }^{[a]}(n m)\end{array}$ & $\begin{array}{l}\mathrm{S}_{B E T}{ }^{[b]} \\
\left(\mathrm{m}^{2} / \mathrm{g}\right)\end{array}$ \\
\hline $\mathrm{Fe} @(N) G$ & 1.18 & 73.06 & 2.44 & - & 2.44 & $5.4 \pm 2.8$ & 252.4 \\
\hline Fe0.46Co0.54@(N)G & 0.50 & 76.55 & 4.10 & 5.15 & 9.25 & $8.6 \pm 4.2$ & 306.6 \\
\hline $\mathrm{Fe}_{0.29} \mathrm{Co}_{0.71} @(\mathrm{~N}) \mathrm{G}$ & 0.51 & 67.02 & 3.82 & 9.92 & 13.74 & $14.4 \pm 7.0$ & 297.3 \\
\hline Co@(N)G & 0.81 & 79.96 & - & 3.06 & 3.06 & $13.1 \pm 5.6$ & 250.6 \\
\hline
\end{tabular}

[a] Calculated from the TEM images; [b] obtained from $\mathrm{N}_{2}$ adsorption for beads of the samples (experimental conditions are provided in Chapter 8) .

In a series of papers, it has been reported that pyrolysis of chitosan renders a carbon residue that upon sonication in different solvents yields $\mathrm{N}$-doped graphene. ${ }^{[35,36]}$ The process has been applied also for chitosan samples containing noble metals ( $\mathrm{Au}, \mathrm{Pt}$ and $\mathrm{Ag}$ ), which, following a similar procedure, yield metal NPs supported on graphene. ${ }^{[37,38]}$ However, in the present case the non-noble metal character of iron and cobalt could lead to formation in some proportion of the corresponding metal carbides. To address this issue, a high resolution XRD pattern was recorded for the $\mathrm{Fe}_{0.29} \mathrm{Co}_{0.71} @(\mathrm{~N}) \mathrm{G}$ sample and the experimental pattern was fitted by the Rietveld refinement. Figure 7.1 presents XRD patterns and the corresponding Rietveld fits of $\mathrm{Fe}_{0.29} \mathrm{Co}_{0.71} @(\mathrm{~N}) \mathrm{G}$, while Figure 7.2 collects XRD patterns measured in a normal method for all samples and Table 7.2 summarizes the results of the analysis for the samples under study. 


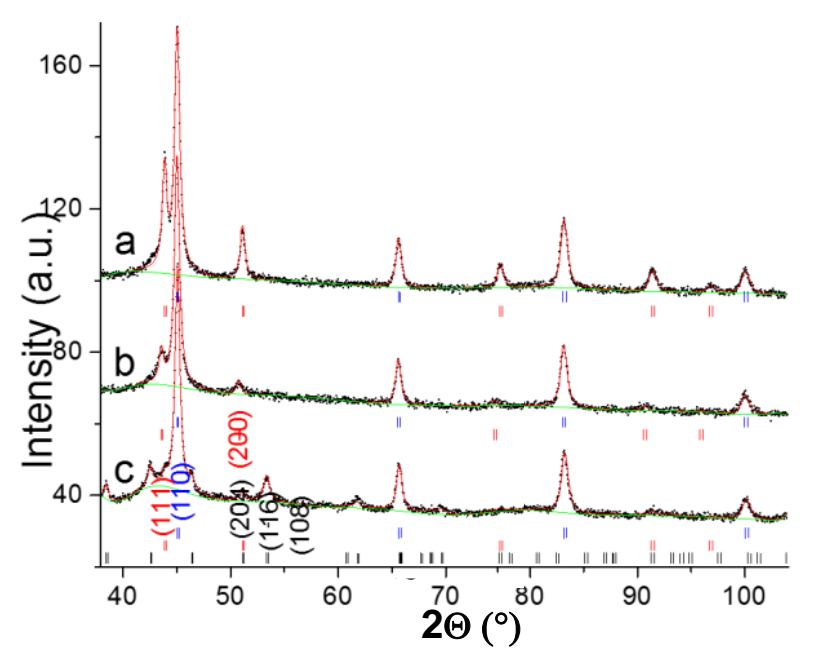

Figure 7.1. Experimental high-resolution XRD pattern of $\mathrm{Fe}_{0.29} \mathrm{Co}_{0.71} @(\mathrm{~N}) \mathrm{G}$ (dots) and the corresponding Rietveld refinement (continuous lines) for as-synthesised sample (a), after $30 \mathrm{~h}$ use as hydrogenation catalysts under the conditions indicated in Table 7.4 (b) and after heating at $550{ }^{\circ} \mathrm{C}$ under $\mathrm{CO}_{2}$ atmosphere (c). The lines in the bottom part of the diffractogram correspond to the position of the peaks of the fcc (red) and bcc (blue) phases of Fe-Co alloy. The contribution of each phase is indicated in Table 7.2 .
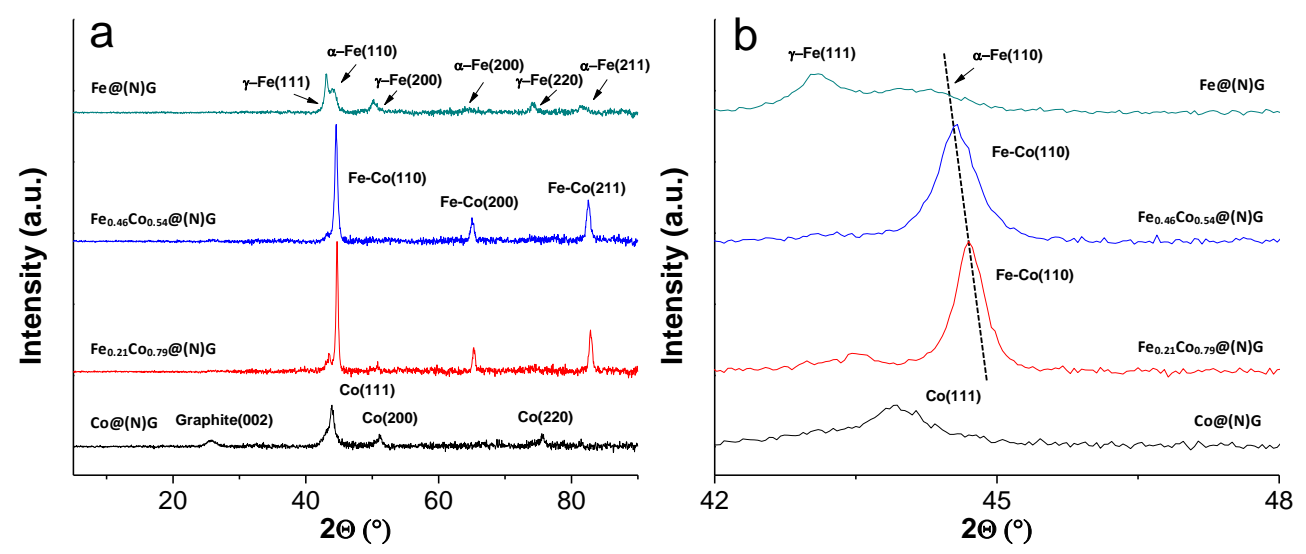

Figure 7.2. XRD patterns of $\mathrm{Fe}_{x} \mathrm{Co}_{y} @(N) G$ samples measured in a normal method in the $2 \theta$ region of $5-90^{\circ}(\mathrm{a})$ and the $2 \theta$ region of $42-48^{\circ}(\mathrm{b})$ where a peak shift at [110] facet is observed, proving the formation of the Fe-Co alloy. 
Table 7.2. Crystallographic phase composition, unit cell parameters and interplanar distance $\left(d_{h k l}\right)$ values obtained from XRD. Weight composition obtained by XRD peak analysis refers only to the metal-containing phases excluding the graphene support.

\begin{tabular}{|c|c|c|c|c|c|c|c|}
\hline Sample & Phase & wt. (\%) & $(\stackrel{\mathrm{a}}{\mathrm{a}})$ & $\begin{array}{c}\mathrm{b} \\
(\AA)\end{array}$ & $\begin{array}{c}\mathrm{c} \\
(\AA)\end{array}$ & (hkl) & $\begin{array}{l}\mathrm{d}_{\mathrm{hkl}} \mathrm{XRD} \\
(\AA)\end{array}$ \\
\hline \multirow{3}{*}{$\mathrm{Fe} @(\mathrm{~N}) \mathrm{G}$} & $\begin{array}{l}\mathrm{Fe}_{3} \mathrm{C} \text { (cementite) } \\
\text { orthorombic Pnma }\end{array}$ & 44.(0) & $5.09(7)$ & $6.76(3)$ & $4.53(0)$ & & \\
\hline & $\begin{array}{c}\gamma \text {-Fe (austenite) } \\
\text { cubic } F m-3 m\end{array}$ & 25.(6) & $3.58(9)$ & & & (111) & 2.073 \\
\hline & $\begin{array}{c}\alpha-\mathrm{Fe} \\
\text { cubic } I m-3 m\end{array}$ & $30 .(4)$ & $2.87(1)$ & & & (110) & 2.030 \\
\hline $\mathrm{Co@(N)G}$ & $\begin{array}{c}\text { Co } \\
\text { cubic } F m-3 m \\
\end{array}$ & 100 & $3.54(7)$ & & & (111) & 2.047 \\
\hline $\mathrm{Fe}_{0.46} \mathrm{Co}_{0.54} @(\mathrm{~N}) \mathrm{G}$ & $\begin{array}{l}\text { FeCo alloy } \\
\text { cubic } P m-3 m\end{array}$ & 100 & $2.85(5)$ & & & (110) & 2.019 \\
\hline \multirow{2}{*}{$\mathrm{Fe}_{0.29} \mathrm{Co}_{0.71} @(\mathrm{~N}) \mathrm{G}$} & $\begin{array}{l}\text { metallic (fcc) phase } \\
\text { cubic } F m-3 m\end{array}$ & $32 .(6)$ & $3.57(2)$ & & & & \\
\hline & $\begin{array}{l}\text { metallic (bcc) phase } \\
\text { cubic } I m-3 m\end{array}$ & 67.(4) & $2.84(5)$ & & & (110) & 2.012 \\
\hline
\end{tabular}

Spontaneous carbochemical reduction of the $\mathrm{Fe}^{3+}$ and $\mathrm{Co}^{2+}$ ions occurs under the reductive conditions of the pyrolysis. There are precedents in the literature reporting this spontaneous reduction for $\mathrm{Ni}^{2+}$ and $\mathrm{Cu}^{2+}$ in the pyrolysis of chitosan. ${ }^{[39]}$ Only in the case of Fe@(N)G the presence of some $\mathrm{Fe}_{3} \mathrm{C}$ carbides (cementite) was observed, the preferential Fe contributors in the sample being cubic $\mathrm{Fm}-3 \mathrm{~m} \mathrm{y}-\mathrm{Fe}$ (austenite) and cubic Im-3m $\alpha$-Fe phase (Table 7.2). In the case of Co@(N)C the presence of $\mathrm{Co}_{2} \mathrm{C}$ was not observed in detectable amounts. The experimental pattern

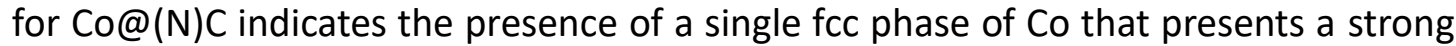
preferential orientation growth in the [111] direction (March-Dollase coefficient $R_{0}$ refined to 0.68 , characteristic for plate-shaped crystals). The [111] plane of the fcc Co phase is a honeycomb lattice of atoms separated by distances of $2.50 \AA$ close to 2.46 $\AA$ corresponding to the distance between the centers of the hexagons of ideal graphene sheet. Such a geometric match could be at the origin of the preferential growth of the [111] plane of Co NPs on the graphene support. A similar claim has been proposed before to justify the preferential growth [111] planes of $\mathrm{Cu}, \mathrm{Au}, \mathrm{Pt}$ 
and Ag nanoplatelets on graphene films. ${ }^{[37,38]}$

The $\mathrm{Fe}_{0.46} \mathrm{Co}_{0.54} @(\mathrm{~N}) \mathrm{G}$ fresh sample contains a single bcc phase of Fe-Co alloy (Table 7.2) without detectable amounts of metal carbides. ${ }^{[40]}$ In contrast, the $\mathrm{Fe}_{0.29} \mathrm{Co}_{0.71} @(\mathrm{~N}) \mathrm{G}$ sample containing a higher Co loading falls in a region of the Fe-Co phase diagram where both bcc and fcc phases coexist. This is also shown by our results (Table 7.2) obtained from Rietveld analysis of the diffraction data (Figure 7.1). The $\mathrm{Fe} / \mathrm{Co}$ distribution between the bcc and fcc phases cannot be determined by Rietveld refinement due to the close values of the atomic scattering factors of Fe and Co (1 $\mathrm{e}^{-}$difference) and the similarity between atomic radii (Fe-1.26 ̊., Co-1.25 ̊̊). ${ }^{[41]}$

The presence and morphology of the metal NPs embedded in a graphitic matrix can be observed by scanning and transmission electron microscopy (SEM and TEM) that also allows to estimate the metal NPs size distribution. Figure 7.3 shows the SEM images of beads of $\mathrm{Fe}_{0.29} \mathrm{Co}_{0.71} @(\mathrm{~N}) \mathrm{G}$ where lots of regular tubes with highly dispersed metal nanoparticles were clearly seen. As we have commented, the formation of the tubes should be due to the contribution of the $\mathrm{CO}_{2}$ supercritical dry to have beads of metal-containing chitosan with porous construction before the pyrolysis. Figure 7.4 shows selected TEM images of $\mathrm{Fe}_{0.29} \mathrm{Co}_{0.71} @(\mathrm{~N}) \mathrm{G}$, while Figures 7.5-7.7 contain a set of TEM images and histograms corresponding to particle size distributions for Fe@(N)G, $\mathrm{Fe}_{0.46} \mathrm{Co}_{0.54} @(\mathrm{~N}) \mathrm{G}, \mathrm{Co} @(\mathrm{~N}) \mathrm{G}$, respectively. From these images it can be determined that there is an important contribution of particles with sizes between 5 and $15 \mathrm{~nm}$ with a few occasionally larger metal NPs (see Table 7.1). These average particle size values for Fe and Co NPs are remarkable considering that the samples have been subjected to heating at $900{ }^{\circ} \mathrm{C}$ for $2 \mathrm{~h}$ and probably reflect the strong metal-graphene interaction. These images show that the metal NPs are embedded into a graphitic matrix formed from chitosan. ${ }^{[35]}$ 


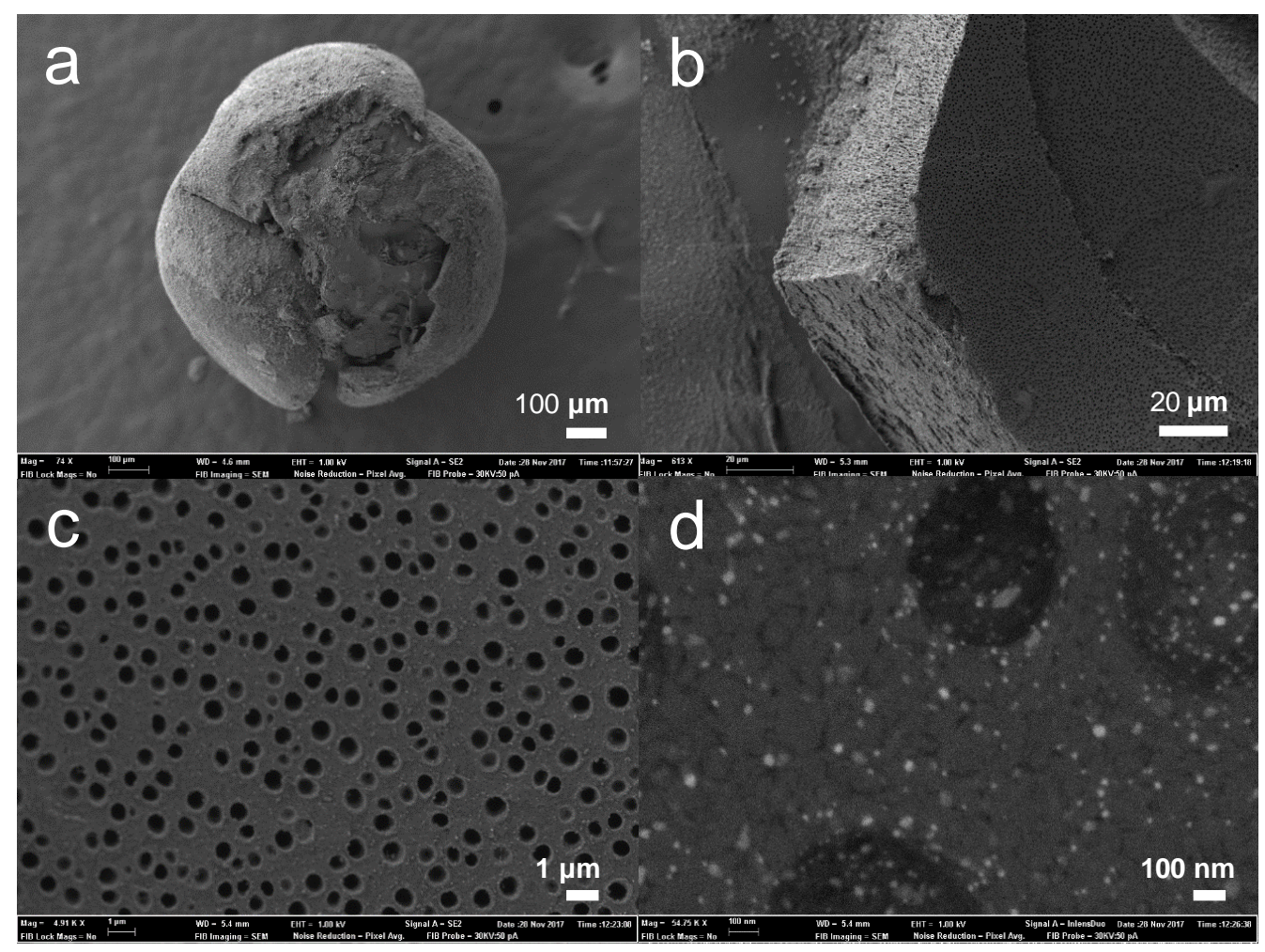

Figure 7.3. (a) SEM image of sphere of $\mathrm{Fe}_{0.29} \mathrm{Co}_{0.71} @(\mathrm{~N}) \mathrm{G}$; (b) cross-section of the wall of the bead; (c,d) SEM images of the front showing the tubes and well dispersed nanoparticles embedded in the inner wall. 


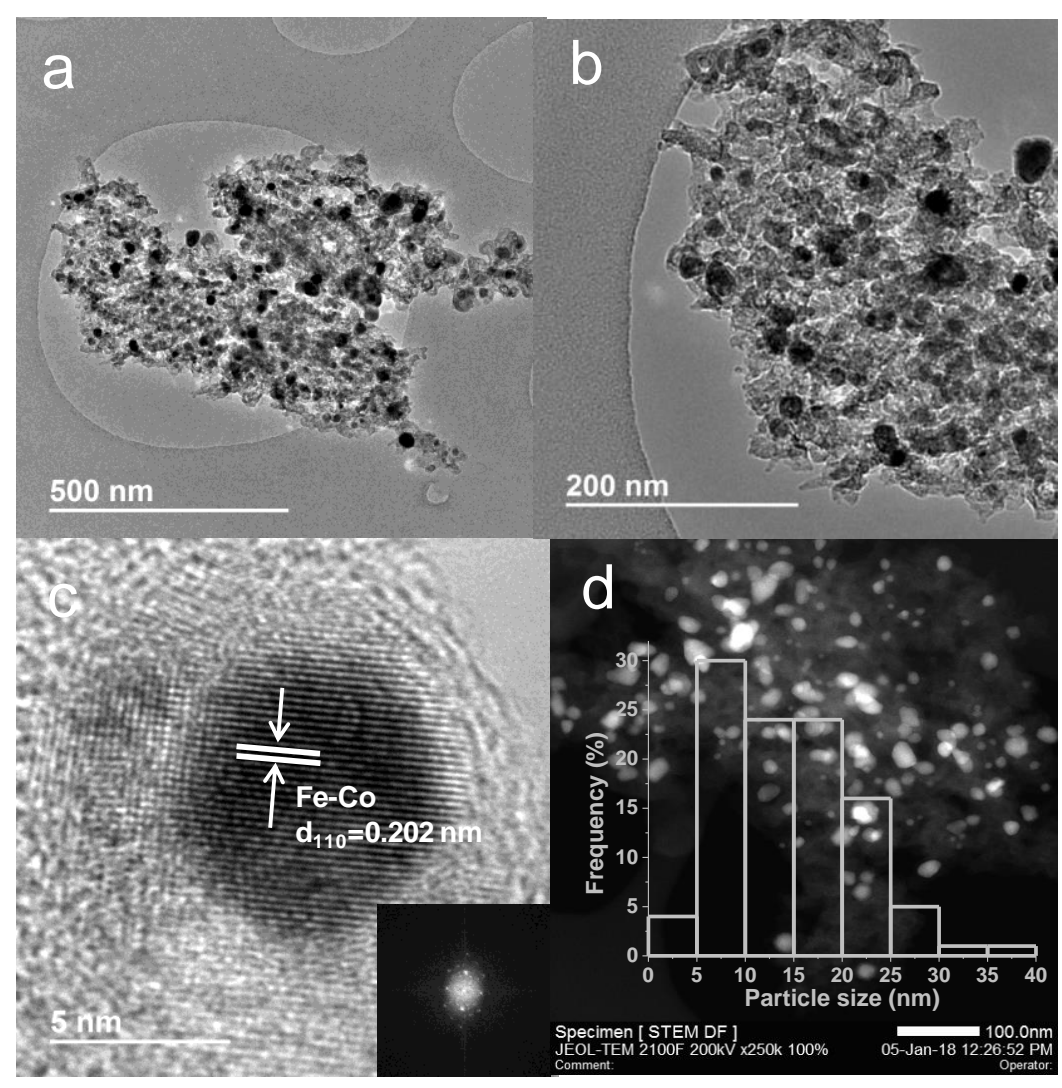

Figure 7.4. $(\mathbf{a}, \mathbf{b}, \mathbf{c})$ Bright-field TEM images at three different magnifications of $\mathrm{Fe}_{0.29} \mathrm{Co}_{0.71} @(\mathrm{~N}) \mathrm{G}$ sample. The interplanar distance for the Fe-Co alloy nanoparticle measured by TEM is indicated in image $\mathbf{c}$. The inset in image c corresponds to the Fast Fourier transformed (FFT) electron diffraction of the particle. (d) Dark-field image of the sample as well as the particle size distribution shown as inset.

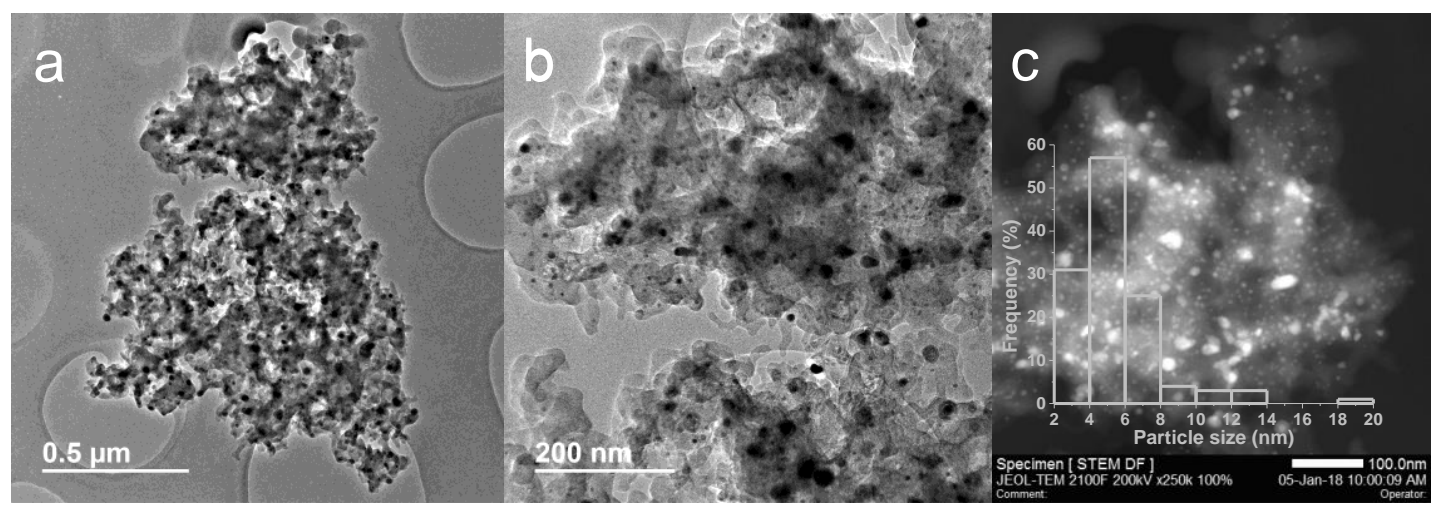

Figure 7.5. (a, b) Bright-field TEM images at two different magnifications of Fe@(N)G sample. (c) Dark-field TEM image of the sample as well as the particle size distribution shown as inset. 


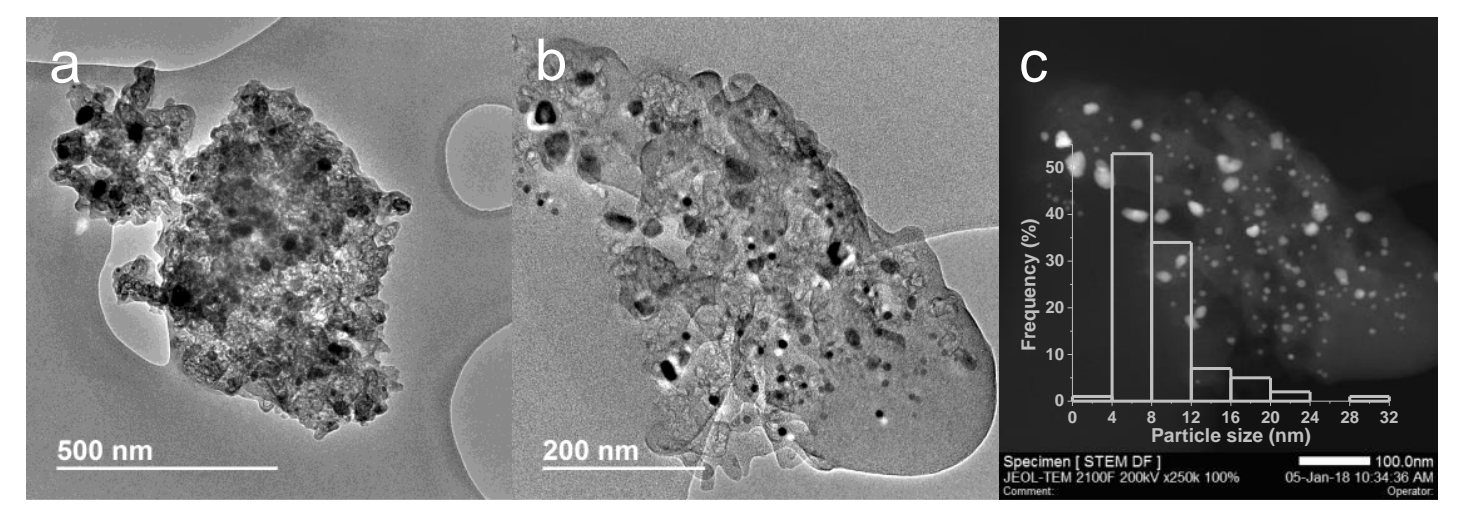

Figure 7.6. (a, b) Bright-field TEM images at two different magnifications of $\mathrm{Fe}_{0.46} \mathrm{Co}_{0.54} @(\mathrm{~N}) \mathrm{G}$ sample. (c) Dark-field TEM image of the sample as well as the particle size distribution shown as inset.

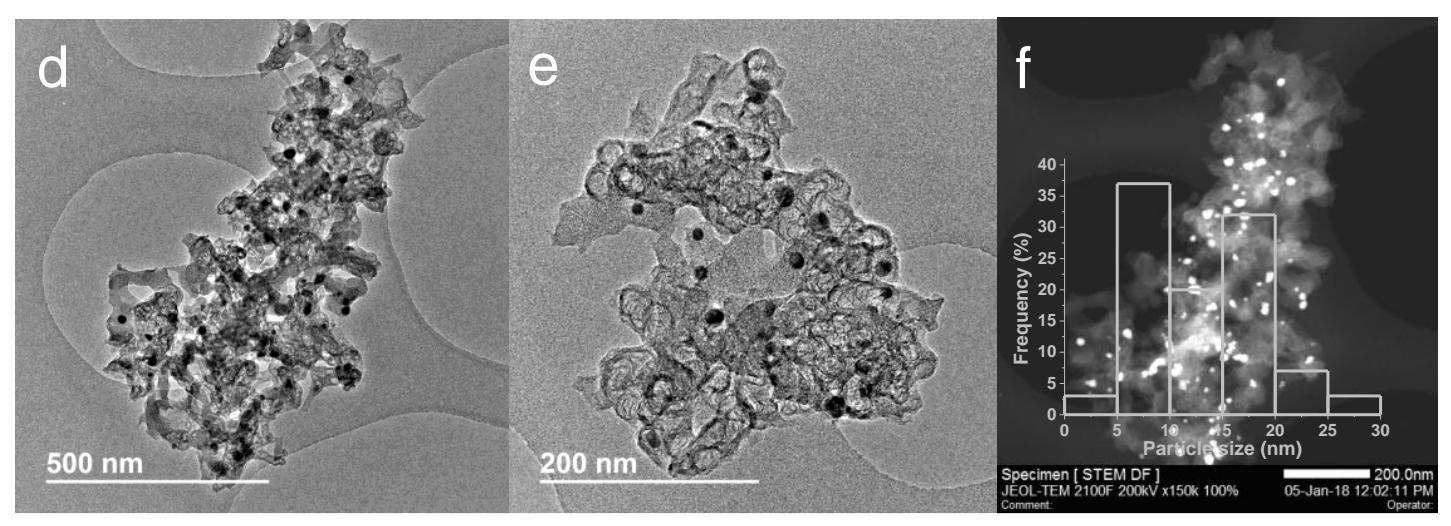

Figure 7.7. (a, b) Bright-field TEM images at two different magnifications of Co@(N)G sample. (c) Dark-field TEM image of the sample used to measure the particle size distribution shown as inset.

EDX analysis as well as elemental C, Fe and Co mapping by TEM for the $\mathrm{Fe}_{0.46} \mathrm{Co}_{0.54} @(\mathrm{~N}) \mathrm{G}$ and $\mathrm{Fe}_{0.29} \mathrm{Co}_{0.71} @(\mathrm{~N}) \mathrm{G}$ samples were performed and the results are provided in Figures 7.8-7.11, respectively. 


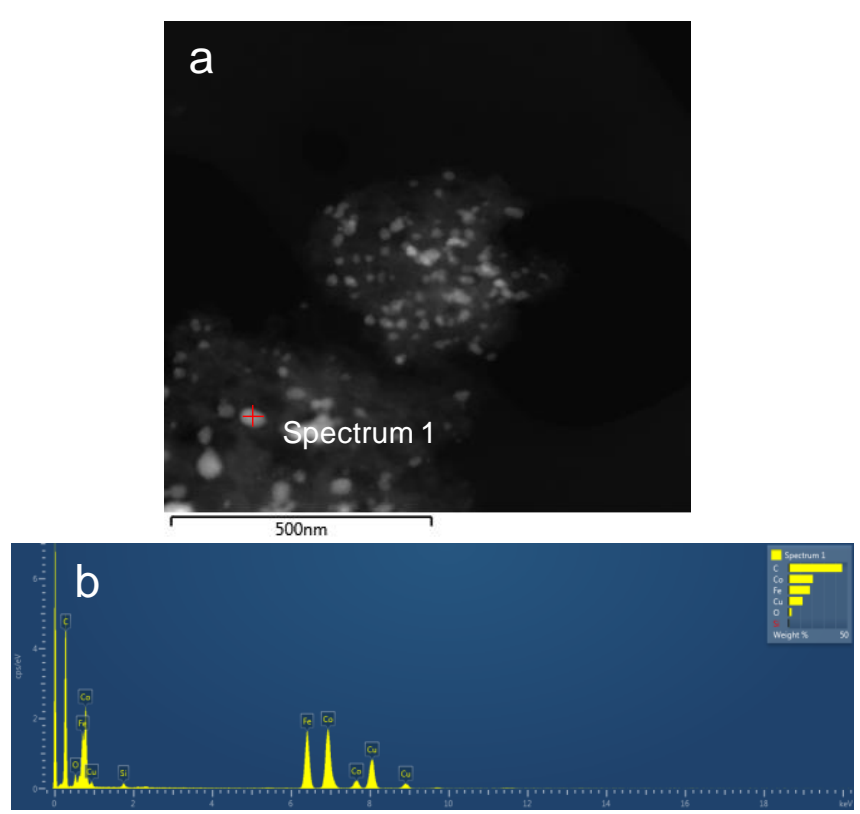

Figure 7.8. TEM image of $\mathrm{Fe}_{0.46} \mathrm{Co}_{0.54} @(\mathrm{~N}) \mathrm{G}$ under dark field (a) and EDX spectrum of the location marked in the image (b). The result shows that Co and Fe are the main metals in the sample with an approximate atomic ratio of 1:1. The strong Cu signal is from the Cu grid.

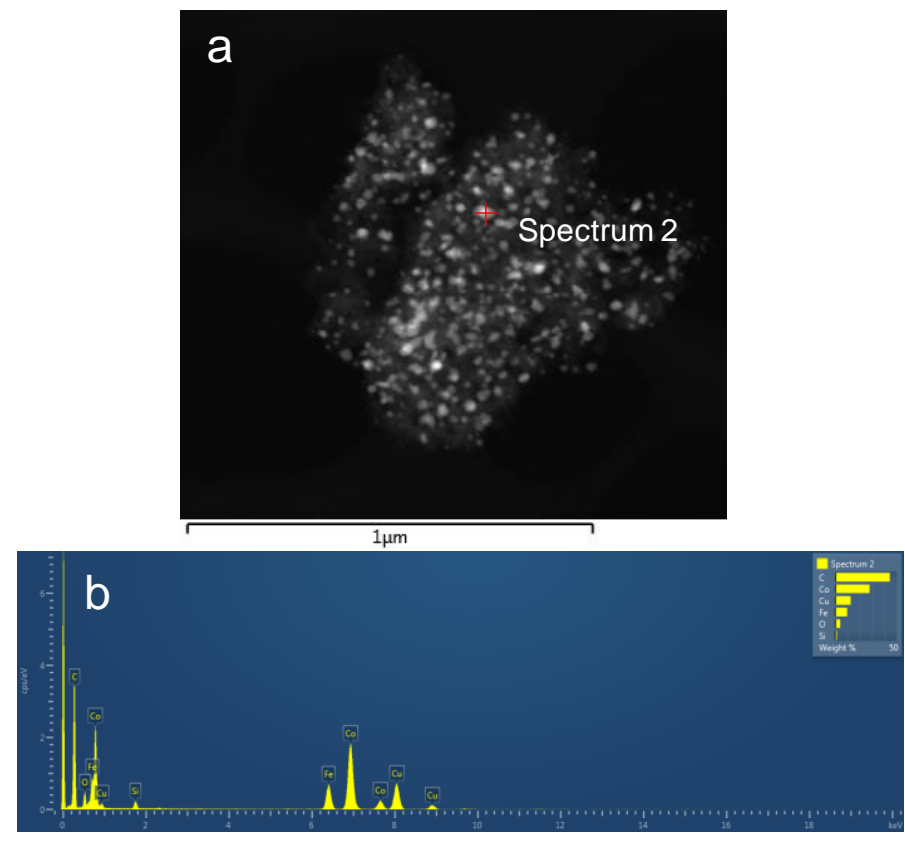

Figure 7.9. TEM image of $\mathrm{Fe}_{0.29} \mathrm{Co}_{0.71} @(\mathrm{~N}) \mathrm{G}$ under dark field (a) and EDX spectrum of the location marked in the image (b). The result shows that $\mathrm{Co}$ and Fe are the main metals in the sample with an approximate atomic ratio of 2:1. The strong $\mathrm{Cu}$ signal is from the Cu grid. 


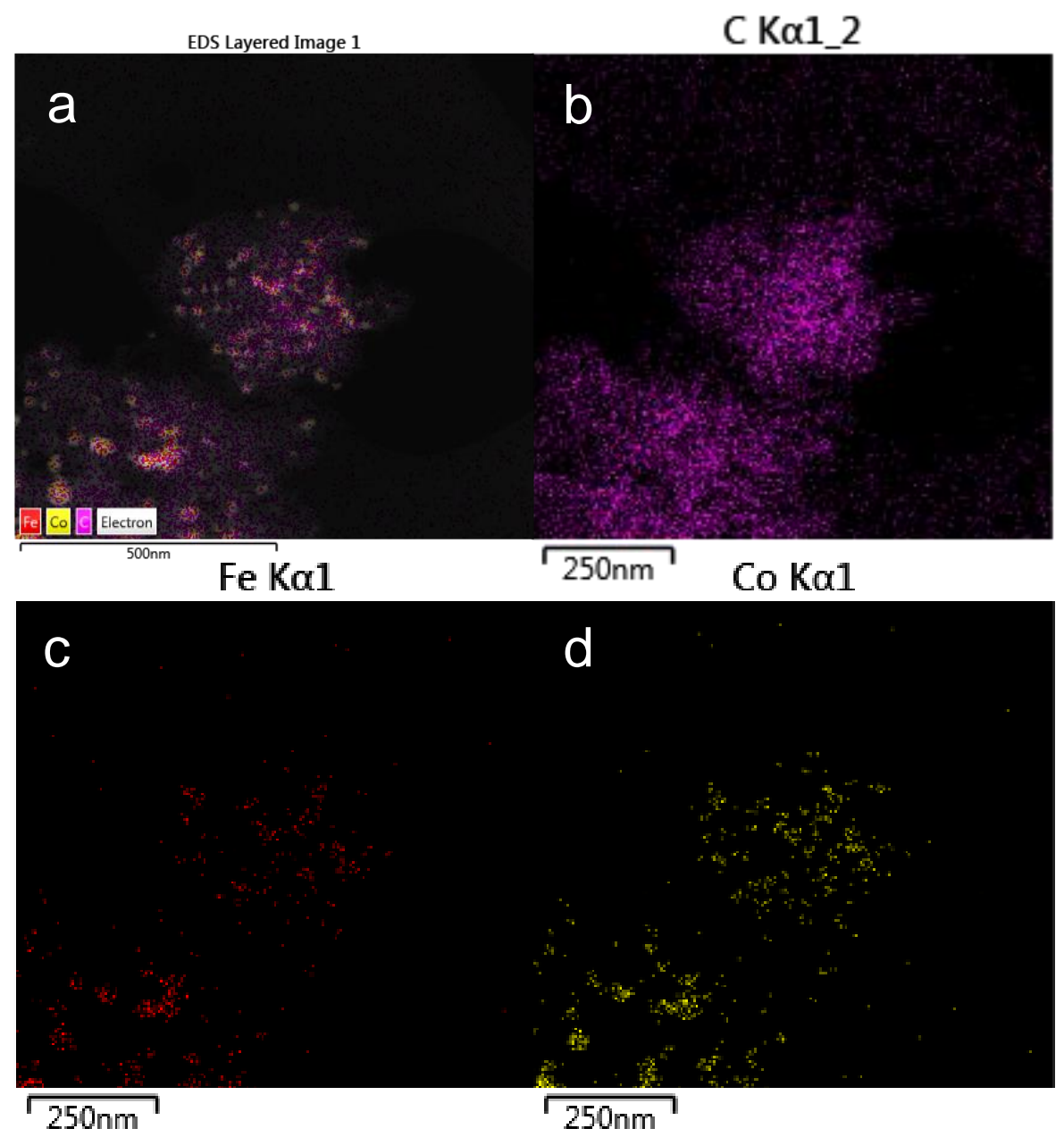

Figure 7.10. Elemental mapping results of $\mathrm{Fe}_{0.46} \mathrm{Co}_{0.54} @(\mathrm{~N}) \mathrm{G}$ indicating the distribution of $\mathrm{C}, \mathrm{Fe}$ and $\mathrm{Co}$ in one image (a) and in separate images (b, c, d) marked with different colors. 


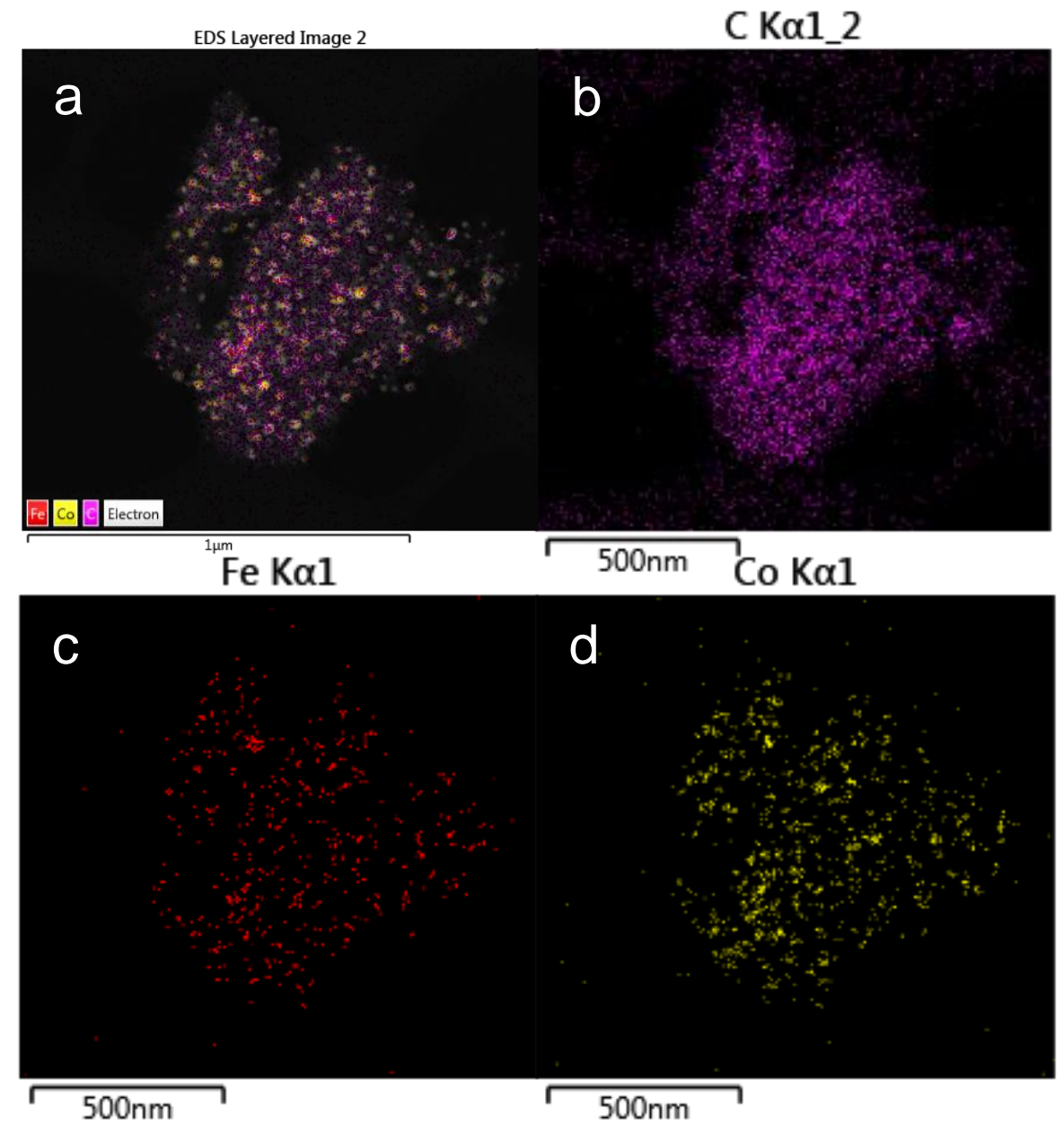

Figure 7.11. Elemental mapping results of $\mathrm{Fe}_{0.29} \mathrm{Co}_{0.71} @(\mathrm{~N}) \mathrm{G}$ indicating the distribution of $\mathrm{C}$, Fe and $\mathrm{Co}$ in one image (a) and in separate images (b, c, d) marked with different colors.

Formation of graphitic carbon during the pyrolysis of chitosan aerogels containing the $\mathrm{Fe}^{3+} / \mathrm{Co}^{2+}$ salts was confirmed by Raman spectroscopy, where the characteristic 2D, G and D peaks appearing at about 2700, 1600 and $1350 \mathrm{~cm}^{-1}$, respectively, were observed. The intensity ratio between the $G$ and the $D$ peak for the samples was about 1.15 that is a normal value for defective $\mathrm{N}$-doped graphene formed for chitosan. ${ }^{[35]}$ The absence of iron or cobalt oxides in the $\mathrm{Fe}_{\mathrm{x}} \mathrm{Co}_{\mathrm{y}} @(\mathrm{~N}) \mathrm{G}$ samples after preparation and prior to their use as catalysts could also be inferred from the absence of the Fe-O and Co-O vibrations peaks appearing below $1000 \mathrm{~cm}^{-1}$ in the low frequency region of the Raman spectra. Figure 7.12 presents the Raman 
spectra of fresh (a) and recovered (b) $\mathrm{Fe}_{0.29} \mathrm{Co}_{0.71} @(\mathrm{~N}) \mathrm{G}$ sample for $\mathrm{CO}_{2}$ hydrogenation, while Figure 7.13 contains the full set of Raman spectra for all as-synthesised samples.

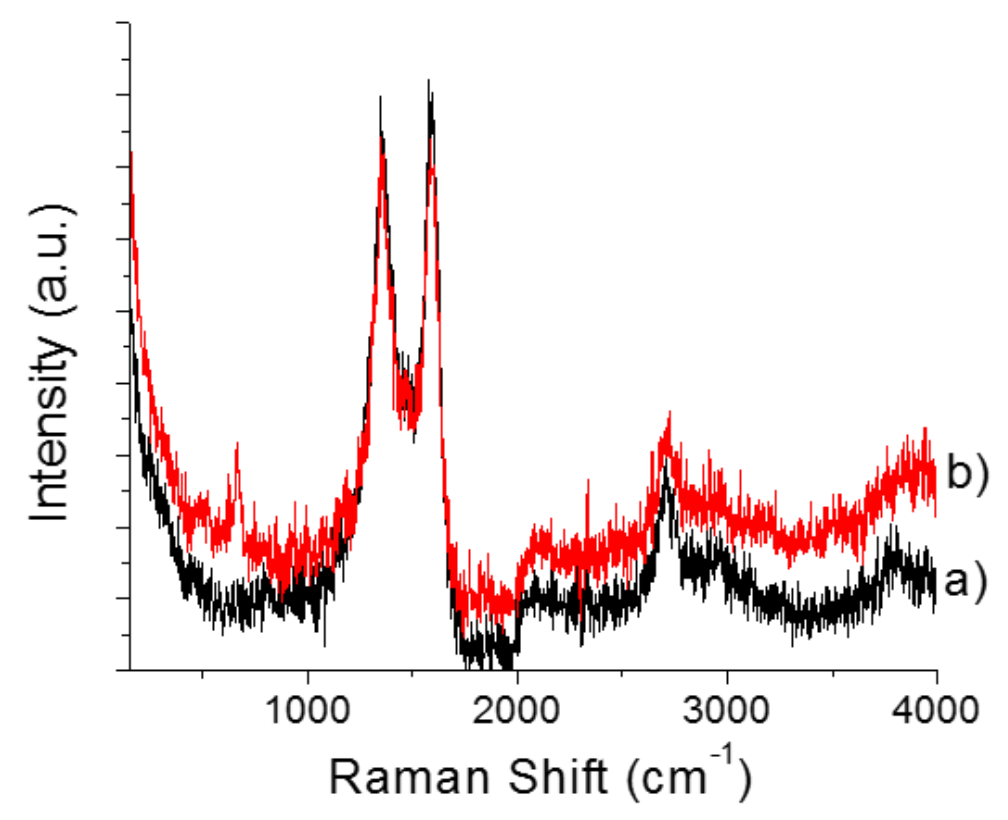

Figure 7.12. Raman spectra recorded with $514 \mathrm{~nm}$ excitation for the as-synthesised

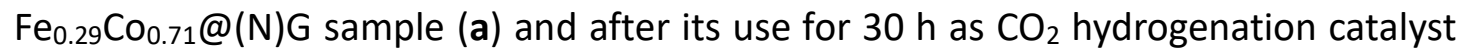
(b) under the conditions indicated in Table 7.4.

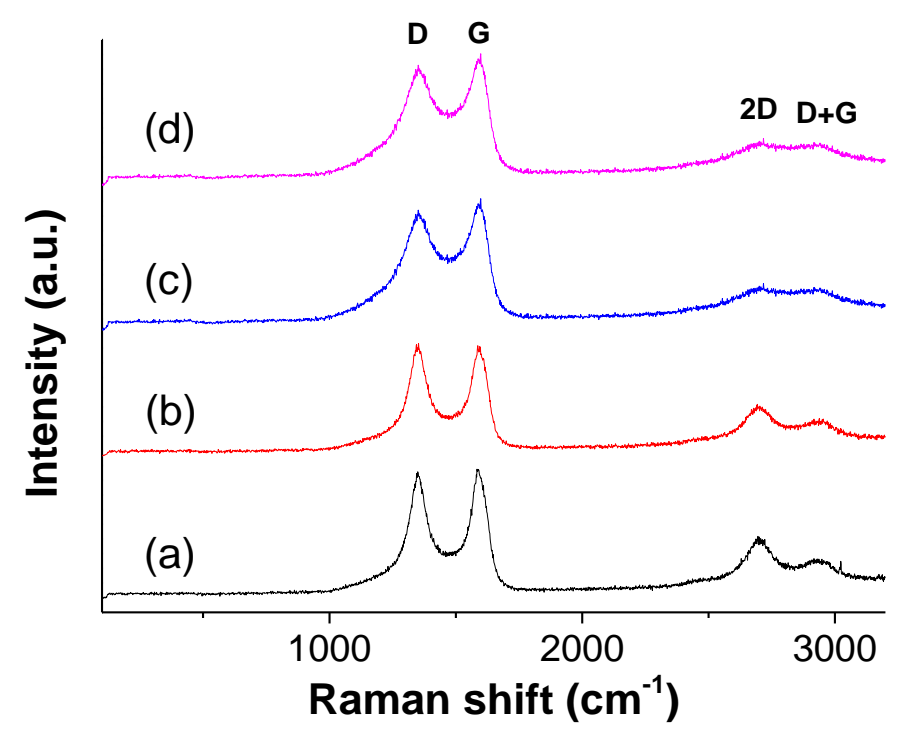

Figure 7.13. Raman spectra recorded with $514 \mathrm{~nm}$ excitation for Co@(N)G (a),

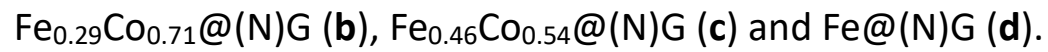


The presence of Fe or Co NPs in the metallic state as well as the predominant graphitic nature of the carbon were also confirmed by XPS, although this specific surface technique reveals the presence of oxidized metals that could not be detected by XRD. Figure 7.14 shows Fe $2 p$, Co $2 p$ and C 1s peaks. Interestingly the presence of some residual $\mathrm{N}$ atoms forming part of a graphene sheet was also detected by XPS, in agreement with elemental analysis and precedents in the literature reporting the formation of $\mathrm{N}$-doped graphene in the pyrolysis of chitosan. ${ }^{[35]}$ Analysis of these spectra indicated that both Fe and Co coexists in various oxidation states. In the case of $\mathrm{Fe}$, the components can be attributed to metallic $\left(706.7 \mathrm{eV}^{[42]}\right)$, Fe carbide (708 $\mathrm{eV}^{[43]}$ ), $\mathrm{FeO}$ (about $710 \mathrm{eV}$ ), $\mathrm{Fe}_{2} \mathrm{O}_{3}$ (between 710-711 $\mathrm{eV}^{[42]}$ ) and $\mathrm{Fe}_{3} \mathrm{O}_{4}$ or over oxidized iron oxide (at $712 \mathrm{eV}^{[44]}$ ). Important to notice, the binding energies of Fe in alloys do not differ much from the values of metallic iron. ${ }^{[45]}$ While for Fe@(N)G the percentage of the reduced iron is in between $51-57$ \%, for $\mathrm{Fe}_{0.46} \mathrm{Co}_{0.54} @(\mathrm{~N}) \mathrm{G}$ and $\mathrm{Fe}_{0.29} \mathrm{Co}_{0.71} @(\mathrm{~N}) \mathrm{G}$ this percentage is significantly lower around 16 and $24 \%$, respectively.

For $\mathrm{Co}$, the species present on the surface according to the analysis of the Co $2 p$ peak are metallic $\left(708 \mathrm{eV}^{[46]}\right), \mathrm{Co}_{3} \mathrm{O}_{4}\left(780 \mathrm{eV}^{[47]}\right)$ and $\mathrm{Co}_{2} \mathrm{O}_{3}\left(781-782 \mathrm{eV}^{[48]}\right)$. The components of binding energy between 783 and $785 \mathrm{eV}$ could also correspond according to the literature ${ }^{[49]}$ to $\mathrm{Co}(\mathrm{CO})_{\times}$species obtained as pyrolysis by-products, while the signal at 787-788 eV corresponds to shake-up characteristic of $\mathrm{Co}_{3} \mathrm{O}_{4}$ and CoO peaks. ${ }^{[49]}$ For $\mathrm{Fe}_{0.46} \mathrm{Co}_{0.54} @(\mathrm{~N}) \mathrm{G}$ and $\mathrm{Fe}_{0.29} \mathrm{Co}_{0.71} @(\mathrm{~N}) \mathrm{G}$ catalysts the shift of the band to $778.5 \mathrm{eV}$ might be an indication of the formation of the Co-Fe alloy. ${ }^{[50]}$ According to literature metallic and cobalt carbide appear at the same binding energy position in the Co2p region. ${ }^{[51]}$ However, this carbide is metastable and its decomposition to metal Co and graphite is thermodynamically favorable. ${ }^{[52]}$

The C1s peaks, presented in Figure $7.14 \mathrm{c}$, feature the main graphene peak, narrow and asymmetric. Although the presence of carbides has been detected in other precedents by observation of a component in the C1s peak at lower binding energy than the typical graphene peak, ${ }^{[52]}$ this component was not observed in the 
XPS spectra of the samples under study.
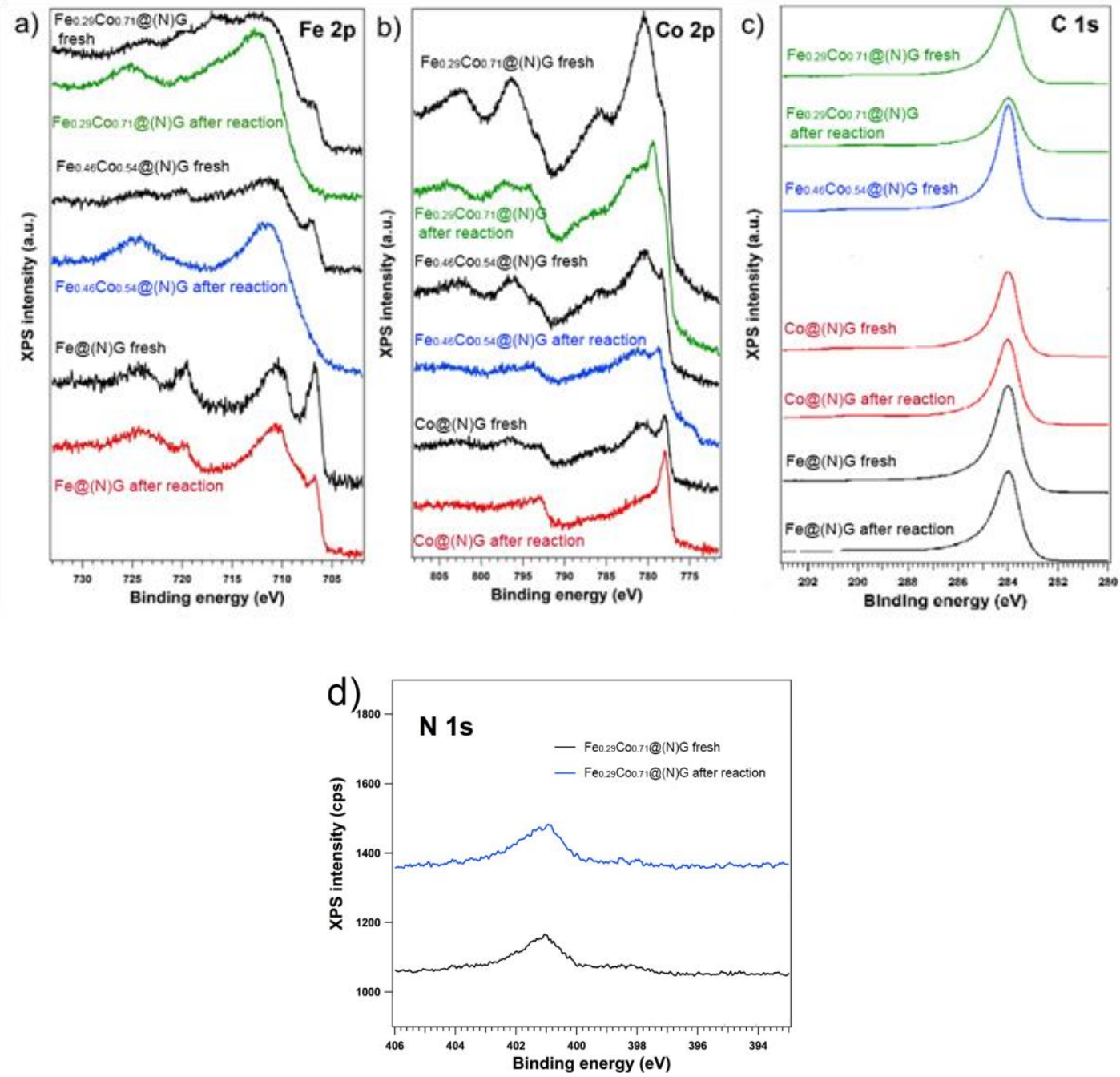

Figure 7.14. X-ray photoelectron spectroscopy (a) Fe 2p; (b) Co 2p; (c) C 1s (d) N 1s for fresh catalysts and after in situ reactions.

Table 7.3 summarizes a comparison of the atomic ratios determined either by XPS or chemical analysis. $\mathrm{Fe}_{0.46} \mathrm{Co}_{0.54} @(\mathrm{~N}) \mathrm{G}$ catalyst shows an enrichment of the surface in Co according XPS data compared with the result from chemical analysis, while for $\mathrm{Fe}_{0.29} \mathrm{Co}_{0.71} @(\mathrm{~N}) \mathrm{G}$ no difference has been determined between the XPS and chemical analysis. For both catalysts, the $\mathrm{Fe} / \mathrm{Co}$ and the total metal $(\mathrm{Fe}, \mathrm{Co}) / \mathrm{C}$ ratios have been not affected by their use as $\mathrm{CO}_{2}$ hydrogenation catalyst for $60 \mathrm{~h}$, providing another proof for the stability of the graphene supported catalysts under the 
catalytic conditions. However, for all catalysts the XPS O/Fe and $\mathrm{O} / \mathrm{Co}$ ratios diminished in the first $2 \mathrm{~h}$ during the reaction and these can be attributed to the reduction of the oxygenated functional groups in graphene under the reaction conditions.

Table 7.3. Comparative XPS and analytic atomic ratios.

\begin{tabular}{|c|c|c|c|c|c|c|}
\hline \multirow[t]{3}{*}{ Catalyst } & \multicolumn{6}{|c|}{ Comparative XPS and analytic atomic ratios } \\
\hline & \multicolumn{5}{|c|}{ XPS } & \multirow{2}{*}{$\begin{array}{l}\text { Chemical } \\
\text { analysis } \\
\mathrm{Fe} / \mathrm{Co}\end{array}$} \\
\hline & $\mathrm{Fe} / \mathrm{Co}$ & $\mathrm{O} / \mathrm{Fe}$ & $\mathrm{O} / \mathrm{Co}$ & $\mathrm{Fe} / \mathrm{C}$ & $\mathrm{Co} / \mathrm{C}$ & \\
\hline Fe@(N)G fresh & & 25.7 & & 0.0017 & & \\
\hline Fe@(N)G post reaction & & 21.4 & & 0.0016 & & \\
\hline Co@(N)G fresh & & & 24.1 & & 0.0015 & \\
\hline Co@(N)G post reaction & & & 20.4 & & 0.0017 & \\
\hline $\mathrm{Fe}_{0.46} \mathrm{Co}_{0.54} @(\mathrm{~N}) \mathrm{G}$ fresh & 0.51 & 26.0 & 13.3 & 0.0014 & 0.0028 & 0.87 \\
\hline $\begin{array}{l}\mathrm{Fe}_{0.46} \mathrm{Co}_{0.54} @(\mathrm{~N}) \mathrm{G} \text { post } \\
\text { reaction }\end{array}$ & 0.47 & 18.6 & 8.8 & 0.0018 & 0.0037 & 0.87 \\
\hline $\mathrm{Fe}_{0.29} \mathrm{Co}_{0.71} @(\mathrm{~N}) \mathrm{Gfresh}$ & 0.42 & 22.0 & 6.5 & 0.0003 & 0.0070 & 0.43 \\
\hline $\begin{array}{l}\mathrm{Fe}_{0.29} \mathrm{Co}_{0.71} @(\mathrm{~N}) \mathrm{G} \text { post } \\
\text { reaction }\end{array}$ & 0.35 & 15.4 & 6.6 & 0.0016 & 0,0051 & 0.43 \\
\hline
\end{tabular}

Accessibility of the metal NPs, even though they are mostly embedded into a carbon matrix, was confirmed by treating these samples with concentrated aqueous $\mathrm{HCl}$ solutions. It was observed that upon treating the samples with this acid, most of the iron or cobalt (above $80 \%$ ) becomes dissolved, although a significant proportion of the Fe and Co still remains on the carbon matrix. These experiments suggest that about $80 \%$ of the total metal content should be considered accessible to interact with substrates and reagents, due to their imperfect wrapping by the graphene matrix, while about $20 \%$ of these NPs would probably not be able to establish direct contact with substrates or reagents.

\subsection{Catalytic activity}

The set of samples prepared containing Fe and/or Co NPs in a carbon matrix 
were initially tested with respect to their catalytic activity for $\mathrm{CO}_{2}$ hydrogenation under continuous flow operation using a $\mathrm{H}_{2} / \mathrm{CO}_{2}$ mol ratio of 3 at 10 bar. Blank experiments in the absence of any catalyst indicate that $\mathrm{CO}_{2}$ conversion at the highest temperature $\left(550^{\circ} \mathrm{C}\right.$ ) was below $6 \%$, methane accompanied by a small percentage of $\mathrm{CO}$ being the only products detected. Another control using graphene in the absence of metal NPs in the same range of temperatures showed some $\mathrm{CO}_{2}$ conversion that increased abruptly in the range of temperatures above $400{ }^{\circ} \mathrm{C}$, being $12.9 \%$ at $500{ }^{\circ} \mathrm{C}$ with a selectivity to methane of $94.5 \%$.

Experiments in the presence of $\mathrm{Fe}_{x} \mathrm{Co}_{y} @(\mathrm{~N}) \mathrm{G}$ samples were carried out using a constant mass of $20 \mathrm{mg}$ of powdered catalyst in the absence of binder and a flow of 1

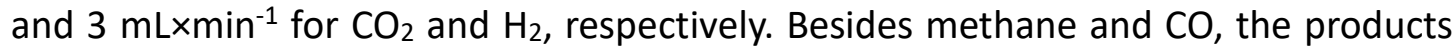
detected in the presence of metallic catalyst were ethane, propane, $i$-butane and methanol in very different proportions depending on the catalyst and reaction conditions. The results achieved in the $\mathrm{CO}_{2}$ hydrogenation by the four samples under study are summarized in Table 7.4.

For each sample, the catalytic tests were performed starting at $300{ }^{\circ} \mathrm{C}$ for $1 \mathrm{~h}$ and increasing the reactor temperature by $50{ }^{\circ} \mathrm{C}$ increments every $1 \mathrm{~h}$ up to $550{ }^{\circ} \mathrm{C}$ and, then, decreasing the temperature by $50^{\circ} \mathrm{C}$ decrements every $1 \mathrm{~h}$ up to returning to $300{ }^{\circ} \mathrm{C}$. Thus, each experiment corresponds to 11 steps of $1 \mathrm{~h}$ of continuous reaction. This sequence allows establishing catalyst stability by comparing conversion and product distribution at a given temperature during the step of increasing and

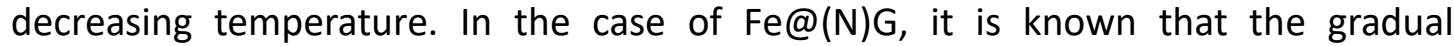
formation of inactive $\theta-\mathrm{Fe}_{3} \mathrm{C}$ phase is the main reason for the catalyst deactivation during $\mathrm{CO}_{2}$ hydrogenation. ${ }^{[53]}$ The initial presence of a high percentage of iron caribide $\left(\theta-\mathrm{Fe}_{3} \mathrm{C}\right)$ in the as-synthesized Fe@(N)G should be responsible for the low catalytic activity in the reaction for synthesis of hydrocarbons. Also, in the present case evidence of the presence of $\chi-\mathrm{Fe}_{5} \mathrm{C}_{2}$ in $\mathrm{Fe} @(\mathrm{~N}) \mathrm{G}$ catalyst after extensive use could not be obtained from XRD peaks analysis. Additionally, when a used $\mathrm{Fe}_{0.46} \mathrm{Co}_{0.54} @(\mathrm{~N}) \mathrm{G}$ sample after a complete evaluation test from 300 to $550{ }^{\circ} \mathrm{C}$ and 
then back to $300{ }^{\circ} \mathrm{C}$ was submitted to a second cycle, a notable coincidence in the conversion and product distribution of this reused sample was observed with respect to the performance of the fresh $\mathrm{Fe}_{0.46} \mathrm{Co}_{0.54} @(\mathrm{~N}) \mathrm{G}$ sample.

As it could be anticipated, Table 7.4 shows that, for the set of four samples under study, $\mathrm{CO}_{2}$ conversion increases with the temperature in the range of 300 to $550{ }^{\circ} \mathrm{C}$. Fe-Co alloys exhibit somewhat higher $\mathrm{CO}_{2}$ conversions than the reference catalysts containing exclusively either $\mathrm{Fe}$ or $\mathrm{Co}$, the highest conversion being measured for $\mathrm{Fe}_{0.29} \mathrm{Co}_{0.71} @(\mathrm{~N}) \mathrm{G}$ that reaches 87 \% under standard conditions. In the case of $\mathrm{Fe} @(\mathrm{~N}) \mathrm{G}$ the main product formed at any temperature was methane in a very high percentage (over $89 \%$ ) and at the highest temperatures close to $100 \%$ selectivity. In contrast, the presence of Co, either as single metallic element or alloyed with Fe, changed completely the selectivity in favor of $i$-butane (Equation 7.1) $i$-Butane selectivity increased with the temperature and for an optimal Fe/Co atomic ratio. In the best present case using $\mathrm{Fe}_{0.29} \mathrm{Co}_{0.71} @(\mathrm{~N}) \mathrm{G}$ as catalyst at $550{ }^{\circ} \mathrm{C}$, a selectivity to $i$-butane over $92 \%$ at a $\mathrm{CO}_{2}$ conversion of $87 \%$ was measured. Comparison of the catalytic performance of the four samples indicates that the outstanding selectivity towards $i$-butane derives from Co, but it increases somewhat, as well as $\mathrm{CO}_{2}$ conversion, with the appropriate proportion of Fe in the alloy.

$$
\mathrm{CO}_{2}+\mathrm{H}_{2} \rightarrow i-\mathrm{C}_{4} \mathrm{H}_{10}+\mathrm{H}_{2} \mathrm{O}
$$

Equation 7.1. The equation for describing the formation of $i$-butane in the $\mathrm{CO}_{2}$ hydrogenation process. 
Chapter 7

Table 7.4. Activity data for the $\mathrm{Fe}_{x} \mathrm{Co}_{y} @(\mathrm{~N}) \mathrm{G}$ samples under study. ${ }^{[a]}$

\begin{tabular}{|c|c|c|c|c|c|c|c|c|c|}
\hline \multirow[b]{2}{*}{$\mathrm{T}\left({ }^{\circ} \mathrm{C}\right)$} & \multicolumn{3}{|c|}{$\mathrm{Fe} @(N) G$} & \multicolumn{3}{|c|}{$\mathrm{Fe}_{0.46} \mathrm{Co}_{054} @(\mathrm{~N}) \mathrm{G}$} & \multicolumn{3}{|c|}{$\begin{array}{c}\mathrm{Fe}_{0.46} \mathrm{Co}_{054} @(\mathrm{~N}) \mathrm{G} \\
-2^{\text {nd }} \text { run (same cat. load) }\end{array}$} \\
\hline & $\begin{array}{l}\text { Conv. } \\
(\%) \mathrm{CO}_{2}\end{array}$ & $\begin{array}{l}\text { Selec.(\%) } \\
i-\mathrm{C}_{4} \mathrm{H}_{10}\end{array}$ & $\begin{array}{l}\text { Selec. } \\
\text { (\%) } \mathrm{CH}_{4}\end{array}$ & $\begin{array}{l}\text { Conv. } \\
\text { (\%) } \mathrm{CO}_{2}\end{array}$ & $\begin{array}{l}\text { Selec. (\%) } \\
i-\mathrm{C}_{4} \mathrm{H}_{10}\end{array}$ & $\begin{array}{l}\text { Selec. } \\
(\%) \mathrm{CH}_{4}\end{array}$ & $\begin{array}{l}\text { Conv. } \\
(\%) \mathrm{CO}_{2}\end{array}$ & $\begin{array}{c}\text { Selec. (\%) } \\
i-\mathrm{C}_{4} \mathrm{H}_{10}\end{array}$ & $\begin{array}{l}\text { Selec. } \\
\text { (\%) } \mathrm{CH}_{4}\end{array}$ \\
\hline 300 & 0.50 & 10.59 & 89.2 & 16.93 & 74.49 & 24.37 & 11.30 & 27.95 & 70.60 \\
\hline 350 & 3.10 & 6.79 & 92.93 & 28.10 & 74.62 & 22.28 & 27.11 & 33.41 & 61.98 \\
\hline 400 & 11.62 & 1.58 & 98.24 & $46.02^{[b]}$ & 61.79 & 31.13 & $\mathbf{4 7 . 4 1 ^ { [ \mathrm { d } ] }}$ & 49.56 & 41.19 \\
\hline 450 & 31.71 & 1.87 & 97.93 & $62.67^{[\mathrm{c}]}$ & 65.15 & 25.44 & 67.50 & 68.99 & 22.09 \\
\hline 500 & 51.55 & 3.01 & 96.79 & 76.30 & 79.96 & 16.61 & 79.45 & 83.36 & 13.72 \\
\hline 550 & 62.10 & 5.95 & 93.73 & 81.16 & 85.58 & 13.32 & 83.33 & 86.52 & 12.51 \\
\hline 500 & 51.73 & 3.08 & 96.71 & 79.96 & 83.13 & 13.83 & 79.26 & 84.10 & 13.10 \\
\hline 450 & 35.00 & 1.92 & 97.86 & 67.13 & 69.12 & 21.85 & 71.11 & 71.20 & 20.68 \\
\hline 400 & 14.72 & 1.13 & 98.65 & 49.96 & 50.99 & 39.40 & 53.93 & 52.15 & 38.88 \\
\hline 350 & 4.88 & 0.06 & 99.77 & 31.15 & 33.35 & 61.66 & 36.75 & 33.02 & 62.32 \\
\hline 300 & 1.25 & 0.25 & 99.75 & 12.95 & 23.63 & 75.07 & 17.07 & 21.08 & 77.26 \\
\hline
\end{tabular}

\begin{tabular}{|c|c|c|c|c|c|c|}
\hline & \multicolumn{3}{|c|}{$\mathrm{Fe}_{0.29} \mathrm{CO}_{0.71} @(\mathrm{~N}) \mathrm{G}$} & \multicolumn{3}{c|}{ Co@(N)G } \\
\hline $\mathrm{T}\left({ }^{\circ} \mathrm{C}\right)$ & $\begin{array}{c}\text { Conv. } \\
(\%) \mathrm{CO}_{2}\end{array}$ & $\begin{array}{c}\text { Selec. } \% \text { ( ) } \\
i-\mathrm{C}_{4} \mathrm{H}_{10}\end{array}$ & $\begin{array}{c}\text { Selec. } \\
(\%) \mathrm{CH}_{4}\end{array}$ & $\begin{array}{c}\text { Conv. } \\
(\%) \mathrm{CO}_{2}\end{array}$ & $\begin{array}{c}\text { Selec. }(\%) \\
i-\mathrm{C}_{4} \mathrm{H}_{10}\end{array}$ & $\begin{array}{c}\text { Selec. } \\
(\%) \mathrm{CH}_{4}\end{array}$ \\
\hline 300 & $\mathbf{1 4 . 8 8}$ & 30.65 & 65.77 & $\mathbf{6 . 5 5}$ & 66.18 & 33.69 \\
\hline 350 & $\mathbf{3 2 . 6 1}$ & 50.01 & 44.66 & $\mathbf{1 8 . 0 3}$ & 60.61 & 39.24 \\
\hline 400 & $\mathbf{5 8 . 5 3}$ & 70.28 & 18.58 & $\mathbf{2 9 . 1 6}$ & 44.79 & 54.88 \\
\hline 450 & $\mathbf{7 7 . 1 2}$ & 84.17 & 9.05 & $\mathbf{5 3 . 7 4}$ & 66.58 & 31.43 \\
\hline 500 & $\mathbf{8 4 . 1 5}$ & 91.44 & 6.80 & $\mathbf{7 4 . 0 8}$ & 84.59 & 14.74 \\
\hline 550 & $\mathbf{8 7 . 1 6}$ & 92.50 & 6.85 & $\mathbf{8 0 . 0 1}$ & 86.86 & 12.90 \\
\hline 500 & $\mathbf{8 5 . 1 6}$ & 92.29 & 6.28 & $\mathbf{6 8 . 4 2}$ & 78.89 & 20.40 \\
\hline 450 & $\mathbf{7 9 . 4 1}$ & 86.91 & 7.31 & $\mathbf{4 6 . 9 4}$ & 58.62 & 39.53 \\
\hline 400 & $\mathbf{6 2 . 4 4}{ }^{[\mathrm{f}, \mathrm{g}]}$ & $\mathbf{7 2 . 8 3}$ & 14.38 & $\mathbf{2 5 . 9 8}$ & 38.16 & 60.65 \\
\hline 350 & $\mathbf{3 6 . 3 4}$ & 51.91 & 37.64 & $\mathbf{1 1 . 7 5}$ & 28.15 & 71.57 \\
\hline 300 & $\mathbf{1 5 . 0 1}$ & 30.31 & 65.27 & $\mathbf{3 . 7 0}$ & 5.20 & 94.78 \\
\hline
\end{tabular}

[a] Reaction conditions: pressure (10 bar), flow rates $\left(\mathrm{H}_{2}: 3 \mathrm{~mL} / \mathrm{min}, \mathrm{CO}_{2}: 1 \mathrm{~mL} / \mathrm{min}\right.$ ), catalyst amount $(20 \mathrm{mg})$. The selectivity (\%) for each other product $\left(\mathrm{CO}, \mathrm{C}_{2} \mathrm{H}_{6}, \mathrm{CH}_{3} \mathrm{OH}\right)$ is below 4.0 except in these cases: [b] 4.38 selectivity (\%) to $\mathrm{C}_{2} \mathrm{H}_{6}$; [c] 4.80 selectivity (\%) to $\mathrm{C}_{2} \mathrm{H}_{6}$; [d] 4.14 selectivity (\%) to $\mathrm{C}_{2} \mathrm{H}_{6}$; [e] 4.02 selectivity (\%) to $\mathrm{CH}_{3} \mathrm{OH}$; [f] 4.82 selectivity (\%) to $\mathrm{CH}_{3} \mathrm{OH}$; [g] 4.57 selectivity (\%) to $\mathrm{C}_{2} \mathrm{H}_{6}$. 
As far as we know, the results presented in Table 7.4 combined with the high catalytic stability for $\mathrm{Fe}$ and Co catalysts are without precedent. In general, hydrocarbon distribution in $\mathrm{CO}_{2}$ hydrogenation by Co catalysts gives methane selectively. ${ }^{[13]}$ Similarly, catalysts based on Fe render a hydrocarbon distribution favoring methane and short hydrocarbons up to $C_{4}$, far from the typical Anderson-Schultz-Flory hydrocarbon distribution generally observed for analogous catalysts for the Fischer-Tropsch hydrogenation of $\mathrm{CO}^{\left[{ }^{[5]}\right.}$ It has been proposed that disfavored $\mathrm{CO}_{2}$ adsorption on the surface of the metal catalyst with respect to that of CO makes the suite of hydrogenation steps of the surface species more favorable than chain growth, resulting in the preferential formation of methane and alkanes with short chain length. ${ }^{[54]}$

Regarding stability, characterization of a $\mathrm{Fe}_{0.29} \mathrm{Co}_{0.71 @(N) G}$ sample after $30 \mathrm{~h}$ operation as catalyst under the conditions of Table 7.4 showed some changes in the phase composition by high resolution $\mathrm{XRD}$, increasing the proportion of the bcc phase to $88 \%$, but without detectable formation of any carbide (Figure 7.1). Raman spectroscopy shows the formation of some iron and cobalt oxides in the catalyst (Figure 7.12), but these species must be only on the surface, since otherwise they would be detectable by XRD as well. Also XPS detects some changes in the shape of the Fe2p and Co2p peaks, probably related to the decrease in the surface oxides as commented earlier (see Table 7.3). However, in the case of the best $\mathrm{Fe}_{0.29} \mathrm{Co}_{0.71} @(\mathrm{~N}) \mathrm{G}$ catalyst, the changes after in situ reaction were much lower than for the other catalysts, and note that XPS is extremely surface sensitive. To confirm catalyst stability, $\mathrm{Fe}_{0.29} \mathrm{Co}_{0.71} @(\mathrm{~N}) \mathrm{G}$ was used as catalyst for an extended period of $500 \mathrm{~h}$ (three weeks) of continuous operation observing that the catalyst maintains over $85 \%$ the activity of the fresh $\mathrm{Fe}_{0.29} \mathrm{Co}_{0.71} @(\mathrm{~N}) \mathrm{G}$ sample, with $i$-butane selectivity still over $90 \%$. 


\subsection{Theoretical calculations}

In order to shed some light on the reasons of the remarkable differences in selectivity and to clarify the role of graphene as support of metallic nanoparticles in the activation of $\mathrm{CO}_{2}$, periodic DFT calculations were undertaken. The differences between the metals $\mathrm{Co}$, Fe and Co-Fe alloy were evaluated using three optimized (Perdew, Burke and Ernzerhof functional, $\mathrm{PBE}^{[55]}$ ) models containing $\mathrm{Co}_{4}$ (G1), $\mathrm{Fe}_{4}(\mathbf{G 2})$ and $\mathrm{CO}_{2} \mathrm{Fe}_{2}(\mathbf{G} 3)$ clusters on the graphene surface (Figure 7.15) in comparison with single metallic clusters (M1, M2 and M3, Figure 7.16).

All the metal clusters supported on graphene proved to be stable due to the occurrence of a favorable interaction between the metal atoms and the graphene model with a characteristic hapticity $(\eta)$ being indicative of a coordination with the aromatic ring of graphene. The calculated hapticity values were $\eta^{4}$ for Co (G1 and G3 clusters) and $\eta^{2}$ and $\eta^{3}$ for Fe (G2 and $\mathbf{G} 3$ clusters, respectively).

(a)
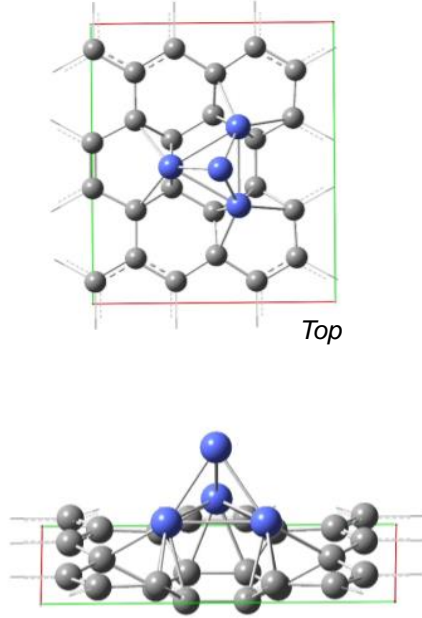

Side (b)
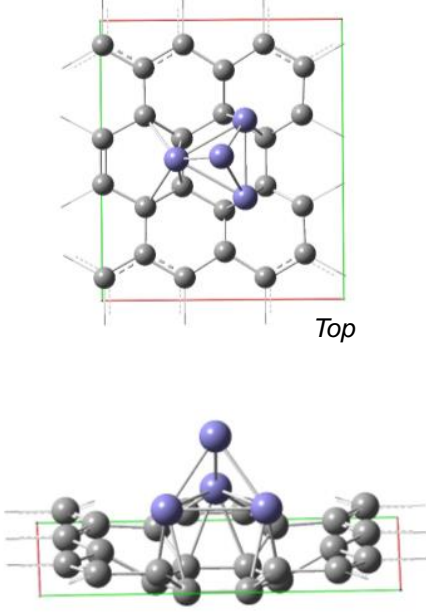

Side (c)
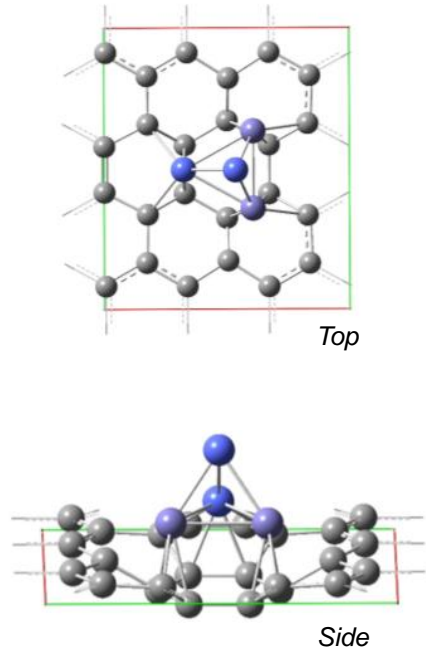

Figure 7.15. Top and lateral views of the optimized models for: (a) $\mathrm{Co}_{4} @ G$ (G1) catalyst; (b) $\mathrm{Fe}_{4} @ G$ (G2) catalyst and (c) $\mathrm{Co}_{2} \mathrm{Fe}_{2} @ G$ (G3) catalyst. Color codes: carbon is gray, cobalt is blue and iron is pale violet. 
(a)

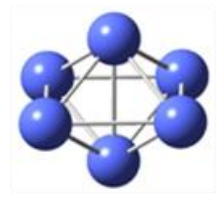

(b)

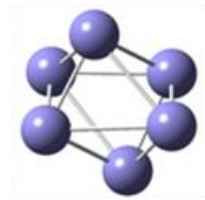

(c)

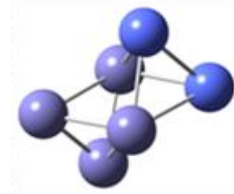

Figure 7.16. Theoretical models for nanoparticles based on metallic clusters: (a) $\mathrm{Co}_{6}$ (M1); (b) $\mathrm{Fe}_{6}$ (M2) and (c) $\mathrm{Co}_{2} \mathrm{Fe}_{4}$ (M3) optimized at PBE level of theory.

The main difference between supported-metals (G1, G2 and G3) and the corresponding clusters (M1, $\mathbf{M} 2$ and $\mathbf{M} 3$ ) arises from the calculated Mulliken charges (Table 7.5). In this context, all three metal atoms attached to the graphene surface have a large positive electron density ( $\delta^{+}$from 0.63 to 1.41 ). However, a different electron density was observed for the atoms which are involved in the activation of both $\mathrm{CO}$ and $\mathrm{CO}_{2}$ species. Thus, the metal atom in $\mathrm{Co}_{4} @ G(G 1)$ and $\mathrm{Fe}_{4} @ G(G 2)$ models binding to $\mathrm{CO}$ or $\mathrm{CO}_{2}$ have a slight negative charge $\left(\delta^{-}=-0.02\right.$ and -0.20 , respectively), contrary to the alloy $\mathrm{Co}_{2} \mathrm{Fe}_{2} @ \mathrm{G}\left(\mathbf{G} 3, \delta^{+}=0.03\right)$. Regarding $\mathbf{M} 1, \mathbf{M} 2$ and M3 clusters, slight variations in electron densities among the four metal atoms were obtained revealing that graphene as platform for nanoparticles is essential for generating more reactive positions.

One important piece of information was obtained from the interaction of these model clusters with $\mathrm{CO}$ and $\mathrm{CO}_{2}$. In both cases, adsorption of these gases was favorable (Figure 7.17). In the case of $\mathrm{CO}$, the computed adsorption energy for the $\mathrm{G} 3$ cluster was $3.2 \mathrm{eV}$ (Figure 7.17a). These calculations also indicate that interaction of Co-Fe alloy supported on graphene with $\mathrm{CO}_{2}$ should provoke its dissociative adsorption, resulting in adsorbed $\mathrm{CO}$ and a highly reactive oxirane species ( $E_{\mathrm{ads}, \mathrm{CO} 2}$ $(G 3)=-4.2 \mathrm{eV}$, Figure 7.17b). This oxirane ring could be involved in the activation of $\mathrm{H}_{2}$ molecules generating $\mathrm{H}-\mathrm{C}-\mathrm{O}$ and $\mathrm{O}-\mathrm{H}$ species on the cluster, and therefore, acting as the first step in the production of $i$-butane. Thus, the results of the modelling support that $\mathrm{CO}_{2}$ hydrogenation in the case of Fe-Co alloys on graphene follows a 
reverse water gas shift mechanism, followed by subsequent Fischer-Tropsch. This proposal would be in agreement with mechanistic data for $\mathrm{CO}_{2}$ hydrogenation on other supported Fe or Co catalysts. ${ }^{[13]}$

Table 7.5. Calculated Mulliken charges for models used herein. Only electron densities of metal cores are shown.

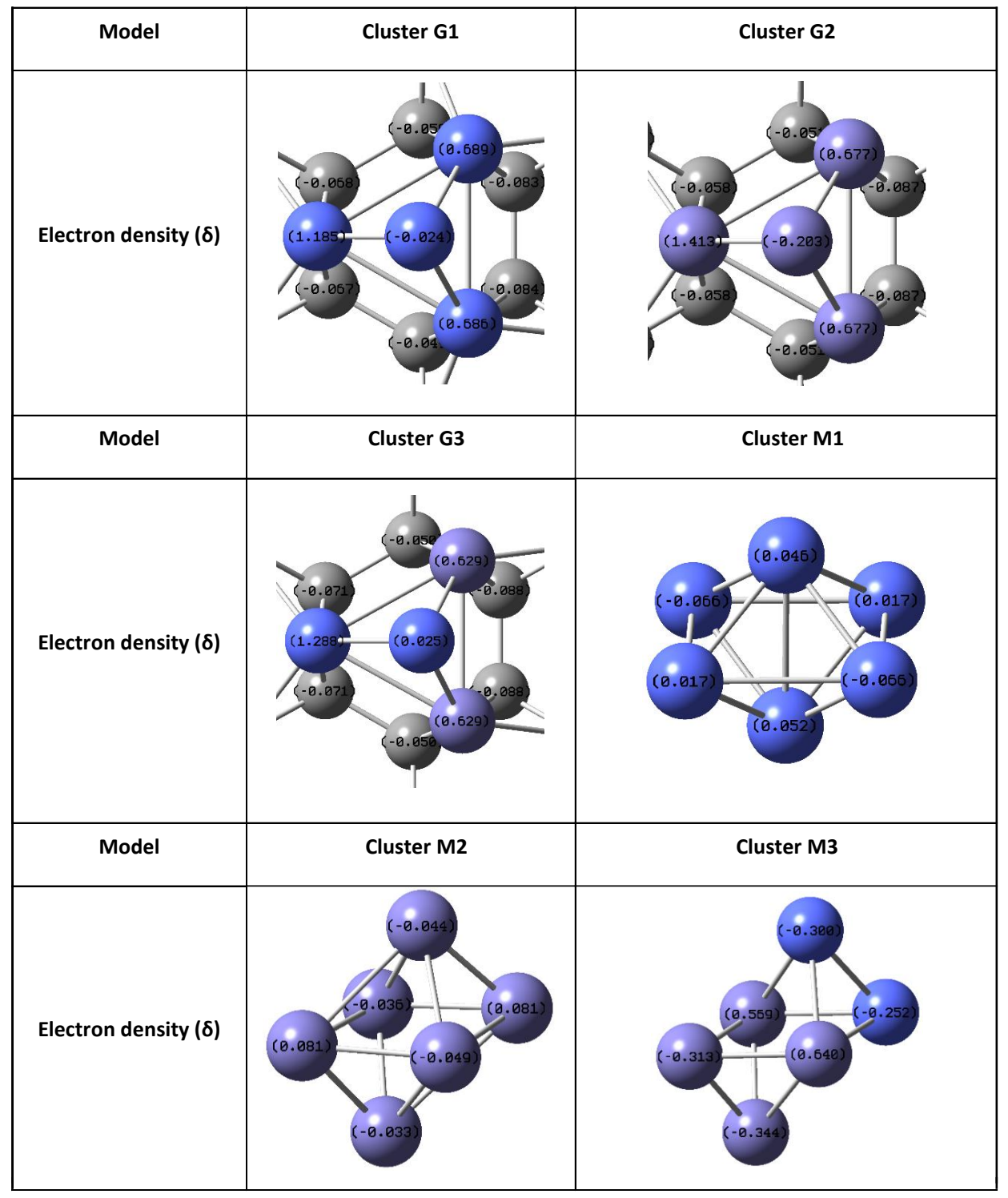


(a)

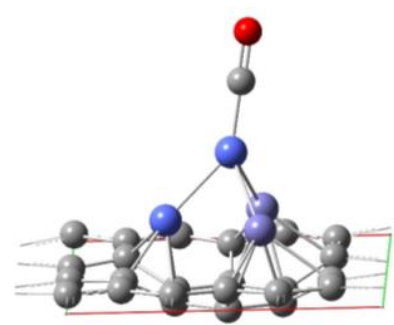

(b)

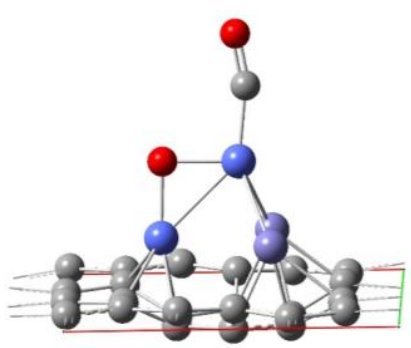

Figure 7.17. PBE-DFT models for adsorption of: (a) carbonyl (CO) group on $\mathrm{Co}_{2} \mathrm{Fe}_{2} @ G$ and (b) dissociative absorption of $\mathrm{CO}_{2}$ on $\mathrm{Co}_{2} \mathrm{Fe}_{2} @ G$. Color codes: carbon is gray, oxygen is red, cobalt is blue and iron is violet pale.

\subsection{Conclusions}

Fe-Co alloy nanoparticles embedded in a $(\mathrm{N})$-doped graphitic carbon matrix prepared by pyrolysis exhibit a strong metal-support interaction that is manifested in the small average particle size. These materials exhibit a remarkable catalytic activity for $\mathrm{CO}_{2}$ hydrogenation, leading those containing $\mathrm{Co}$ to an unprecedented outstanding selectivity to $i$-butane over $92 \%$ at $87 \% \mathrm{CO}_{2}$ conversion in some cases. This catalytic activity is in sharp contrast with the current state of the art in which methane is the prevalent product, generally with selectivity values over $70 \%$. $i$-Butane is a much more valuable product than methane and, therefore, the present results open an unforeseen avenue on the control of $\mathrm{CO}_{2}$ hydrogenation towards other products higher than $\mathrm{C} 1$. Theoretical calculations indicate that the interaction between the metals and graphene, resulting in a charge transfer and differentiation among the charge density of the various atoms in the cluster, is the main reason for this remarkable behavior. 


\subsection{References}

[1] Nakicenovic, N.; Alcamo, J.; Grubler, A.; Riahi, K.; Roehrl, R.; Rogner, H. H.; Victor, N. Special report on emissions scenarios (SRES), a special report of Working Group III of the intergovernmental panel on climate change; Cambridge University Press, 2000.

[2] Edmonds, J.; Wise, M.; Barns, D. W. Carbon coalitions: The cost and effectiveness of energy agreements to alter trajectories of atmospheric carbon dioxide emissions. Energy Policy 1995, 23(4-5), 309-335.

[3] Hecht, A. D.; Tirpak, D. Framework agreement on climate change: A scientific and policy history. Climatic Change 1995, 29(4), 371-402.

[4] Schellnhuber, H. J.; Rahmstorf, S.; Winkelmann, R. Why the right climate target was agreed in Paris. Nature Climate Change 2016, 6(7), 649.

[5] Parmesan, C.; Yohe, G. A globally coherent fingerprint of climate change impacts across natural systems. Nature 2003, 421(6918), 37-42.

[6] Rayner, N. A.; Parker, D. E.; Horton, E. B.; Folland, C. K.; Alexander, L. V.; Rowell, D. P.; Kent, E. C.; Kaplan, A. Global analyses of sea surface temperature, sea ice, and night marine air temperature since the late nineteenth century. Journal of Geophysical ResearchAtmospheres 2003, 108(D14).

[7] Cox, P. M.; Betts, R. A.; Jones, C. D.; Spall, S. A.; Totterdell, I. J. Acceleration of global warming due to carbon-cycle feedbacks in a coupled climate model. Nature 2000, 408(6809), 184-187.

[8] Aresta, M.; Dibenedetto, A. Utilisation of $\mathrm{CO}_{2}$ as a chemical feedstock: opportunities and challenges. Dalton Transactions 2007, (28), 2975-2992.

[9] Song, C. S. Global challenges and strategies for control, conversion and utilization of $\mathrm{CO}_{2}$ for sustainable development involving energy, catalysis, adsorption and chemical processing. Catalysis Today 2006, 115(1-4), 2-32.

[10] Sakakura, T.; Choi, J. C.; Yasuda, H. Transformation of carbon dioxide. Chemical Reviews 2007, 107(6), 2365-2387.

[11] Arakawa, H.; Aresta, M.; Armor, J. N.; Barteau, M. A.; Beckman, E. J.; Bell, A. T.; Bercaw, J. E.; Creutz, C.; Dinjus, E.; Dixon, D. A.; Domen, K.; DuBois, D. L.; Eckert, J.; Fujita, E.; 
Gibson, D. H.; Goddard, W. A.; Goodman, D. W.; Keller, J.; Kubas, G. J.; Kung, H. H.; Lyons, J. E.; Manzer, L. E.; Marks, T. J.; Morokuma, K.; Nicholas, K. M.; Periana, R.; Que, L.; Rostrup-Nielson, J.; Sachtler, W. M. H.; Schmidt, L. D.; Sen, A.; Somorjai, G. A.; Stair, P. C.; Stults, B. R.; Tumas, W. Catalysis research of relevance to carbon management: Progress, challenges, and opportunities. Chemical Reviews 2001, 101(4), 953-996.

[12] Aresta, M.; Dibenedetto, A.; Angelini, A. Catalysis for the valorization of exhaust carbon: from $\mathrm{CO}_{2}$ to chemicals, materials, and fuels. Technological use of $\mathrm{CO}_{2}$. Chemical Reviews 2014, 114(3), 1709-1742.

[13] Wang, W.; Wang, S.; Ma, X.; Gong, J. Recent advances in catalytic hydrogenation of carbon dioxide. Chemical Society Reviews 2011, 40(7), 3703-3727.

[14] Schlogl, R. The revolution continues: energiewende 2.0. Angewandte Chemie -International Edition 2015, 54(15), 4436-4439.

[15] Dry, M. E. The Fischer-Tropsch process: 1950-2000. Catalysis Today 2002, 71(3-4), 227-241.

[16] Van der Laan, G. P.; Beenackers, A. Kinetics and selectivity of the Fischer-Tropsch synthesis: A literature review. Catalysis Reviews-Science and Engineering 1999, 41(3-4), 255-318.

[17] Li, S.; Li, A.; Krishnamoorthy, S.; Iglesia, E. Effects of Zn, Cu, and K promoters on the structure and on the reduction, carburization, and catalytic behavior of iron-based Fischer-Tropsch synthesis catalysts. Catalysis Letters 2001, 77(4), 197-205.

[18] Ning, W. S.; Koizumi, N.; Chang, H.; Mochizuki, T.; Itoh, T.; Yamada, M. Phase transformation of unpromoted and promoted Fe catalysts and the formation of carbonaceous compounds during Fischer-Tropsch synthesis reaction. Applied Catalysis A-General 2006, 312, 35-44.

[19] Tu, J. I.; Ding, M. Y.; Zhang, Q.; Zhang, Y. I.; Wang, C. G.; Wang, T. J.; Ma, L. I.; Li, X. J. Design of carbon-encapsulated $\mathrm{Fe}_{3} \mathrm{O}_{4}$ nanocatalyst with enhanced performance for Fischer-Tropsch synthesis. ChemCatChem 2015, 7(15), 2323-2327.

[20] Chen, W.; Fan, Z.; Pan, X.; Bao, X. Effect of confinement in carbon nanotubes on the activity of Fischer-Tropsch iron catalyst. Journal of the American Chemical Society 2008, 130(29), 9414-9419. 
[21] Chew, L. M.; Ruland, H.; Schulte, H. J.; Xia, W.; Muhler, M. $\mathrm{CO}_{2}$ hydrogenation to hydrocarbons over iron nanoparticles supported on oxygen-functionalized carbon nanotubes. Journal of Chemical Sciences 2014, 126(2), 481-486.

[22] Minett, D. R.; O'Byrne, J. P.; Pascu, S. I.; Plucinski, P. K.; Owen, R. E.; Jones, M. D.; Mattia, D. Fe@CNT-monoliths for the conversion of carbon dioxide to hydrocarbons: structural characterisation and Fischer-Tropsch reactivity investigations. Catalysis Science \& Technology 2014, 4(9), 3351-3358.

[23] Lux, L.; Williams, K.; Ma, S. Q. Heat-treatment of metal-organic frameworks for green energy applications. Crystengcomm 2015, 17(1), 10-22.

[24] Shen, K.; Chen, X. D.; Chen, J. Y.; Li, Y. W. Development of MOF-derived carbon-based nanomaterials for efficient catalysis. ACS Catalysis 2016, 6(9), 5887-5903.

[25] Jang, Y. H.; Kochuveedu, S. T.; Jang, Y. J.; Shin, H. Y.; Yoon, S.; Steinhart, M.; Kim, D. H. The fabrication of graphitic thin films with highly dispersed noble metal nanoparticles by direct carbonization of block copolymer inverse micelle templates. Carbon 2011, 49(6), 2120-2126.

[26] Zhang, Z. P.; Qin, Y. S.; Dou, M. L.; Ji, J.; Wang, F. One-step conversion from Ni/Fe polyphthalocyanine to $\mathrm{N}$-doped carbon supported $\mathrm{Ni}$-Fe nanoparticles for highly efficient water splitting. Nano Energy 2016, 30, 426-433.

[27] He, J. B.; Dhakshinamoorthy, A.; Primo, A.; Garcia, H. Iron nanoparticles embedded in graphitic carbon matrix as heterogeneous catalysts for the oxidative $\mathrm{C}-\mathrm{N}$ coupling of aromatic N-H compounds and amides. Chemcatchem 2017, 9(15), 3003-3012.

[28] Wu, G.; Nelson, M.; Ma, S.; Meng, H.; Cui, G.; Shen, P. K. Synthesis of nitrogen-doped onion-like carbon and its use in carbon-based CoFe binary non-precious-metal catalysts for oxygen-reduction. Carbon 2011, 49(12), 3972-3982.

[29] Aijaz, A.; Masa, J.; Rösler, C.; Xia, W.; Weide, P.; Botz, A. J.; Fischer, R. A.; Schuhmann, W.; Muhler, M. Co@ $\mathrm{Co}_{3} \mathrm{O}_{4}$ encapsulated in carbon nanotube-grafted nitrogen-doped carbon polyhedra as an advanced bifunctional oxygen electrode. Angewandte Chemie International Edition 2016, 55(12), 4087-4091.

[30] Bhatnagar, A.; Sillanpaa, M. Applications of chitin- and chitosan-derivatives for the detoxification of water and wastewater - A short review. Advances in Colloid and Interface. 
Science 2009, 152(1-2), 26-38.

[31] Jing, G. H.; Wang, L.; Yu, H. J.; Amer, W. A.; Zhang, L. Recent progress on study of hybrid hydrogels for water treatment. Colloids and Surfaces a-Physicochemical and Engineering Aspects 2013, 416, 86-94.

[32] Primo, A.; Quignard, F. Chitosan as efficient porous support for dispersion of highly active gold nanoparticles: design of hybrid catalyst for carbon-carbon bond formation. Chemical Communications 2010, 46(30), 5593-5595.

[33] Primo, A.; Liebel, M.; Quignard, F. o. Palladium coordination biopolymer: A versatile access to highly porous dispersed catalyst for suzuki reaction. Chemistry of Materials 2009, 21(4), 621-627.

[34] Molvinger, K.; Quignard, F.; Brunel, D.; Boissière, M.; Devoisselle, J.-M. Porous chitosan-silica hybrid microspheres as a potential catalyst. Chemistry of Materials 2004, 16(17), 3367-3372.

[35] Primo, A.; Sanchez, E.; Delgado, J. M.; Garcia, H. High-yield production of N-doped graphitic platelets by aqueous exfoliation of pyrolyzed chitosan. Carbon 2014, 68, 777-783.

[36] Rizescu, C.; Podolean, I.; Albero, J.; Parvulescu, V. I.; Coman, S. M.; Bucur, C.; Puche, M.; Garcia, H. N-doped graphene as a metal-free catalyst for glucose oxidation to succinic acid. Green Chemistry 2017, 19(8), 1999-2005.

[37] Esteve-Adell, I.; Bakker, N.; Primo, A.; Hensen, E.; Garcia, H. Oriented Pt nanoparticles supported on few-layers graphene as highly active catalyst for aqueous-phase reforming of ethylene glycol. ACS Applied Materials \& Interfaces 2016, 8(49), 33690-33696.

[38] Mateo, D.; Esteve-Adell, I.; Albero, J.; Royo, J. F. S.; Primo, A.; Garcia, H. 111 oriented gold nanoplatelets on multilayer graphene as visible light photocatalyst for overall water splitting. Nature Communications 2016, 7, 11819.

[39] Abellan, G.; Latorre-Sanchez, M.; Fornes, V.; Ribera, A.; Garcia, H. Graphene as a carbon source effects the nanometallurgy of nickel in $\mathrm{Ni}, \mathrm{Mn}$ layered double hydroxide-graphene oxide composites. Chemical Communications 2012, 48(93), 11416-11418.

[40] Wang, J.; Lu, X. G.; Zhu, N.; Zheng, W. Thermodynamic and diffusion kinetic studies of the Fe-Co system. Calphad 2017, 58, 82-100. 
[41] Wells, A. F. Structural inorganic chemistry; Oxford university press, 2012.

[42] Hawn, D. D.; DeKoven, B. M. Deconvolution as a correction for photoelectron inelastic energy losses in the core level XPS spectra of iron oxides. Surface and Interface Analysis 1987, 10(2-3), 63-74.

[43] Shabanova, I.; Trapeznikov, V. A study of the electronic structure of $\mathrm{Fe}_{3} \mathrm{C}, \mathrm{Fe}_{3} \mathrm{Al}$ and $\mathrm{Fe}_{3} \mathrm{Si}$ by X-ray photoelectron spectroscopy. Journal of Electron Spectroscopy and Related Phenomena 1975, 6(4), 297-307.

[44] Den Daas, H.; Passacantando, M.; Lozzi, L.; Santucci, S.; Picozzi, P. The interaction of $\mathrm{Cu}(100) / F e$ surfaces with oxygen studied by X-ray photoelectron spectroscopy. Surface Science 1994, 317(3), 295-302

[45] Kishi, K.; Nishioka, J. Interaction of $\mathrm{Fe} / \mathrm{Cu}(100)$, Fe-Ni/Cu (100) and Ni/Fe/Cu (100) surfaces with $\mathrm{O}_{2}$ studied by XPS. Surface Science 1990, 227(1-2), 97-106.

[46] McIntyre, N.; Cook, M. X-ray photoelectron studies on some oxides and hydroxides of cobalt, nickel, and copper. Analytical Chemistry 1975, 47(13), 2208-2213.

[47] McIntyre, N.; Johnston, D.; Coatsworth, L.; Davidson, R.; Brown, J. X-ray photoelectron spectroscopic studies of thin film oxides of cobalt and molybdenum. Surface and Interface Analysis 1990, 15(4), 265-272.

[48] Tan, B. J.; Klabunde, K. J.; Sherwood, P. M. XPS studies of solvated metal atom dispersed (SMAD) catalysts. Evidence for layered cobalt-manganese particles on alumina and silica. Journal of the American Chemical Society 1991, 113(3), 855-861.

[49] Artyushkova, K.; Levendosky, S.; Atanassov, P.; Fulghum, J. XPS Structural studies of nano-composite non-platinum electrocatalysts for polymer electrolyte fuel cells. Topics in Catalysis 2007, 46(2-3), 263-275.

[50] Turner, N.; Single, A. Determination of peak positions and areas from wide-scan XPS spectra. Surface and Interface Analysis 1990, 15(3), 215-222.

[51] Müller, U.; Falub, C.; Thorwarth, G.; Voisard, C.; Hauert, R. Diamond-like carbon coatings on a CoCrMo implant alloy: A detailed XPS analysis of the chemical states at the interface. Acta Materialia 2011, 59(3), 1150-1161.

[52] Rodriguez-Gomez, A.; Holgado, J. P.; Caballero, A. Cobalt carbide identified as catalytic site for the dehydrogenation of ethanol to acetaldehyde. ACS Catalysis 2017, 7(8), 
$5243-5247$.

[53] Lee, S. C.; Kim, J. S.; Shin, W. C.; Choi, M. J.; Choung, S. J. Catalyst deactivation during hydrogenation of carbon dioxide: Effect of catalyst position in the packed bed reactor. Journal of Molecular Catalysis A: Chemical 2009, 301(1-2), 98-105.

[54] Visconti, C. G.; Lietti, L.; Tronconi, E.; Forzatti, P.; Zennaro, R.; Finocchio, E. Fischer-Tropsch synthesis on a $\mathrm{Co} / \mathrm{Al}_{2} \mathrm{O}_{3}$ catalyst with $\mathrm{CO}_{2}$ containing syngas. Applied Catalysis A: General 2009, 355(1-2), 61-68.

[55] Perdew, J. P.; Burke, K.; Ernzerhof, M. Generalized gradient approximation made simple. Physical Review Letters 1996, 77(18), 3865. 
Chapter 8

\section{Experimental section}

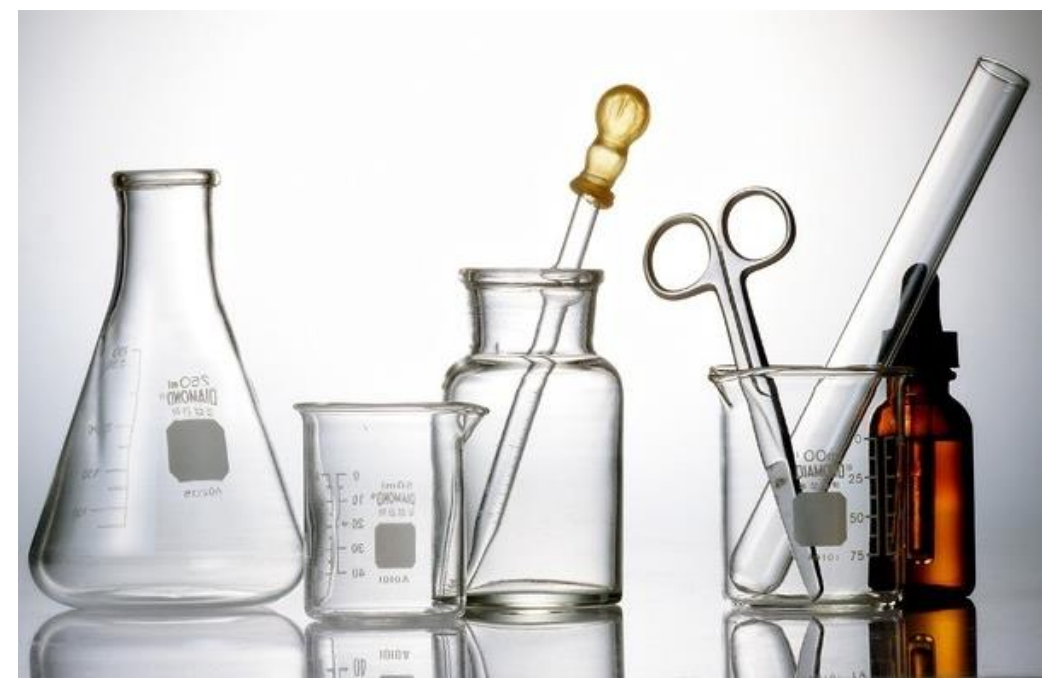





\subsection{Synthesis of materials}

\subsubsection{Synthesis of (N)G films}

Chitosan $(250 \mathrm{mg}$ ) from Aldrich was dissolved in a $12.5 \mathrm{~mL}$ aqueous solution. $250 \mu \mathrm{L}$ of HOAc solution was added to dissolve chitosan completely. After $2 \mathrm{~h}$ under magnetic string at room temperature, the solutions were filtered through a syringe of $0.45 \mu \mathrm{m}$ pore size to remove the impurities present in the commercial chitosan. The films were prepared on a previously cleaned quartz plate $\left(2 \times 2 \mathrm{~cm}^{2}\right)$ by casting $500 \mu \mathrm{L}$ of the filtered chitosan solution at $4000 \mathrm{rpm}$ in $45 \mathrm{~s}$. Once dried on a hot plate, the films were pyrolyzed under $\mathrm{Ar}$ flow or $\mathrm{Ar} / \mathrm{H}_{2}$ (5 vol\%) or $\mathrm{Ar} / \mathrm{H}_{2}$ (10 vol\%) flow (200 mL $\mathrm{min}^{-1}$ ), increasing the temperature at a rate of $5^{\circ} \mathrm{C} \mathrm{min}^{-1}$ up to $900,1000,1100$ and $1200{ }^{\circ} \mathrm{C}$, respectively, and holding the final temperature for $1 \mathrm{~h}$. The sample was allowed to cool at room temperature under inert atmosphere by stopping electrical heating.

\subsubsection{Synthesis of $G$ films}

Alginic acid (800 mg) from Aldrich was dissolved in a $10 \mathrm{~mL}$ aqueous solution. $1.6 \mathrm{~mL}$ of $\mathrm{NH}_{4} \mathrm{OH}$ solution (28-30\% $\mathrm{NH}_{3}$ in water) was added to assist dissolution of alginic acid completely. After $2 \mathrm{~h}$ under magnetic string at room temperature, the solutions were filtered through a syringe of $0.45 \mu \mathrm{m}$ pore size to remove the impurities present in the commercial alginic acid. The films were prepared on a previously cleaned quartz plate $\left(2 \times 2 \mathrm{~cm}^{2}\right)$ by casting $500 \mu \mathrm{L}$ of filtered solution at $4000 \mathrm{rpm}$ in $45 \mathrm{~s}$. Once dried on a hot plate, the films were pyrolyzed under Ar flow or $\mathrm{Ar} / \mathrm{H}_{2}$ (5 vol\%) or $\mathrm{Ar} / \mathrm{H}_{2}$ (10 vol\%) flow $\left(200 \mathrm{~mL} \mathrm{~min}{ }^{-1}\right)$, increasing the temperature at a rate of $5{ }^{\circ} \mathrm{C} \min ^{-1}$ up to $900,1000,1100$ and $1200{ }^{\circ} \mathrm{C}$, respectively, holding the final temperature for $1 \mathrm{~h}$. The sample was allowed to cool at room temperature 
under inert atmosphere by stopping electrical heating.

\subsubsection{Synthesis of (N)G/h-BN and h-BN}

In a general preparation procedure, chitosan (1.12 g) from Aldrich was dissolved in $25 \mathrm{~mL}$ of aqueous solution contained $\left(\mathrm{NH}_{4}\right)_{3} \mathrm{BO}_{3}(0.45 \mathrm{~g})$. After $2 \mathrm{~h}$ of magnetic stirring at room temperature, the solutions were filtered through syringe of $0.45 \mu \mathrm{m}$ pore size to remove possible insoluble particles present in the commercial chitosan. Then, the films were deposited on a previously cleaned quartz plate $\left(2 \times 2 \mathrm{~cm}^{2}\right)$ by spin casting $500 \mu \mathrm{L}$ of filtered solution at $4000 \mathrm{rpm}$ in $45 \mathrm{~s}$. Once dried on a hot plate, the $\left(\mathrm{NH}_{4}\right)_{3} \mathrm{BO}_{3}$-chitosan films were pyrolyzed under $\operatorname{Ar}$ flow $(200 \mathrm{~mL} \mathrm{~min}-1$ ), increasing the temperature at a rate of $0.9{ }^{\circ} \mathrm{C} \mathrm{min}^{-1}$ up to $900{ }^{\circ} \mathrm{C}$ and holding for $2 \mathrm{~h}$. To get the h-BN films, the pyrolyzed films (graphene/h-BN) were calcined up to $1000{ }^{\circ} \mathrm{C}$ in air with a heating rate of $8{ }^{\circ} \mathrm{C} \mathrm{min}^{-1}$ to remove the defective graphene layer. Thicker layers were obtained by decreasing the volume of the aqueous solution to $10 \mathrm{~mL}$.

\subsubsection{Synthesis of oriented $\mathrm{MoS}_{2} / \mathrm{ml}-\mathrm{G}$ films}

Alginic acid (1200 mg) from Aldrich (St. Louis, MO, USA) was suspended in aqueous solutions containing different concentrations of $\left(\mathrm{NH}_{4}\right)_{2} \mathrm{MoS}_{4}(0.5,1,2,3,5$, 10 or $60 \mathrm{mM}$ ). Two milliliters of $\mathrm{NH}_{4} \mathrm{OH}$ solution (28-30\% $\mathrm{NH}_{3}$ in water) were added to dissolve alginic acid completely. After $2 \mathrm{~h}$ under magnetic stirring at room temperature, the solutions were filtered through a syringe of $0.45 \mu \mathrm{m}$ pore size to remove insoluble impurities possibly present in commercial alginic acid. The films were cast on a previously cleaned quartz plate $\left(2 \times 2 \mathrm{~cm}^{2}\right)$ by spin coating $500 \mu \mathrm{L}$ of filtered ammonium alginate solution at $4000 \mathrm{rpm}$ for $45 \mathrm{~s}$. Once dried on a hot plate, the films were pyrolyzed under Ar flow $\left(200 \mathrm{~mL} \mathrm{~min}^{-1}\right)$, increasing the temperature at a rate of $5^{\circ} \mathrm{C} \mathrm{min}^{-1}$ up to $900{ }^{\circ} \mathrm{C}$ and a holding time of $1 \mathrm{~h}$. After this time, the films were cooled down at room temperature maintaining the Ar flow. 


\subsubsection{Synthesis of Fe/CoNP@C}

Briefly, Fe-Co ${ }_{1.42} \mathrm{NP} @ \mathrm{C}, \mathrm{Fe}-\mathrm{Co}_{0.86} \mathrm{NP} @ \mathrm{C}$ were prepared by mixing chitosan (400 $\mathrm{mg}$ ) in a $20 \mathrm{~mL}$ aqueous solution of $\mathrm{Fe}(\mathrm{OAc})_{2}(20.5 \mathrm{mg})$ and $\mathrm{Co}(\mathrm{OAc})_{2} \cdot \mathrm{H}_{2} \mathrm{O}(20.0 \mathrm{or}$ $28.3 \mathrm{mg}$ for preparation of each of the two samples). HOAc $(250 \mu \mathrm{l})$ was added to dissolve chitosan completely. Samples of FeNP@C and CoNP@C were prepared following the same procedure, but using only $\mathrm{Fe}(\mathrm{OAc})_{2}(20.5 \mathrm{mg}$ ) or $\mathrm{Co}(\mathrm{OAc})_{2} \cdot \mathrm{H}_{2} \mathrm{O}(28.3 \mathrm{mg})$, respectively. After $2 \mathrm{~h}$ under magnetic string at room temperature, the solutions were dried by removing the water by heating the solution at $70{ }^{\circ} \mathrm{C}$ overnight. The resulting samples were pyrolyzed under Ar flow $(200 \mathrm{~mL}$ $\left.\mathrm{min}^{-1}\right)$, increasing the temperature at a rate of $10{ }^{\circ} \mathrm{C} \mathrm{min}^{-1}$ up to $900{ }^{\circ} \mathrm{C}$ with a holding time of $2 \mathrm{~h}$. The samples were allowed to cool at room temperature under inert atmosphere by stopping electrical heating.

\subsubsection{Synthesis of $\mathrm{Fe}_{x} \mathrm{Co}_{y} @(N) G$}

Briefly, $\mathrm{Fe}_{0.46} \mathrm{Co}_{0.54} @(\mathrm{~N}) \mathrm{G}$ and $\mathrm{Fe}_{0.21} \mathrm{Co}_{0.79} @(\mathrm{~N}) \mathrm{G}$ were prepared by mixing chitosan $(1000 \mathrm{mg})$ in a $50 \mathrm{ml}$ aqueous solution of $\mathrm{Fe}(\mathrm{OAc})_{3}(75 \mathrm{mg})$ and $\mathrm{Co}(\mathrm{OAc})_{2}(75$ or $150 \mathrm{mg}$, respectively). $625 \mu \mathrm{L}$ HAc was added to dissolve chitosan completely. Fe@(N)G and Co@(N)G were prepared following the same produce, but using only $\mathrm{Fe}(\mathrm{OAc})_{3}(75 \mathrm{mg})$ or $\mathrm{Co}(\mathrm{OAc})_{2}(75 \mathrm{mg})$. After that, the solutions were added dropwise to a $\mathrm{NaOH}$ solution $(0.1 \mathrm{M})$ by using a syringe $(0.8 \mathrm{~mm}$ needle diameter) with a slow stirring at the same time. The gel microspheres were formed on the bottom of the solution. After immersing them in the solution for $1 \mathrm{~h}$, the gel microspheres were washed with distilled water. The resulting hydrogel beads were then immersed into a series of ethanol/water baths with an increasing concentration of ethanol $(10,30,50$, 70,90 and 100 vol\%, respectively) for 15 minutes each. The beads were dried in the condition of superficial $\mathrm{CO}_{2}$ with a pressure of above 75 bar and a temperature of $40{ }^{\circ} \mathrm{C}$ for 20 minutes to yield aerogel beads. The resulting beads were then pyrolyzed under Ar flow (200 mL/min), increasing the temperature at a rate of $1^{\circ} \mathrm{C} / \mathrm{min}$ up to 
$200{ }^{\circ} \mathrm{C}$ for $2 \mathrm{~h}$ and then to $900{ }^{\circ} \mathrm{C}$ with a holding time of $2 \mathrm{~h}$. The beads were finally grinded into powders for some characterizations and catalytic tests.

\subsection{Experimental procedures}

\subsubsection{Photoelectronic tests for (N)G and G films used as electrodes}

The experiments were carried out in a quartz cylindrical three-electrode cell on a Versastat electrochemical workstation with an (N)G or G photoelectrode as the working electrode, a platinum foil as the counter electrode and $\mathrm{Ag} / \mathrm{AgCl}$ (saturated $\mathrm{KCl}$ ) as the reference electrode. The working electrode was irradiated using a LED lamp as the light source through an optical fibre that indicates the photoelectrode from the front. Line-sweep voltammgrams were measured at a scan rate of $50 \mathrm{mV} / \mathrm{s}$ in an aqueous solution of $1 \mathrm{M} \mathrm{LiClO}_{4}$ which had been previously purged with argon gas for 30 mins. The photoelectric experiments were performed with a positive $1.1 \mathrm{~V}$ applied bias, and the production of $\mathrm{H}_{2}$ envolved in the photoreactor was determined by gas chromatography using GS-MOL column of 15 meters and $0.55 \mathrm{~mm}$ ID with TCD.

\subsubsection{Electrical measurements for (N)G/h-BN and (N)G films}

The current-voltage curves were measured with a home-made Kelvin probe system. One of the electrical contacts of the sample was done by evaporating metallic gold through a shadow mask in a specific region of the sample. The second contact was made with a tungsten probe tip with a radius of $0.5 \mu \mathrm{m}$ (PTG20-0.5, Microword). The position of the tungsten probe was controlled with a nanopositioner. The electrical measurements were carried out using a Keithley 2450 Sourcemeter 
with a resolution of $f A$ and using low noise triaxial cables. The scan rate was $200 \mathrm{mV} / \mathrm{s}$. The charge accumulated was calculated by subtracting the difference of the integrated area in the forward and reverse bias with respect at the scan rate in the first quadrant.

\subsubsection{Electrochemical measurements for oriented $\mathrm{MoS}_{2} / \mathrm{ml}-\mathrm{G}$ films}

Electrocatalytic measurements of $\overline{\mathrm{MoS}_{2}} / \mathrm{ml}-\mathrm{G}$ electrodes were carried out using a potentiostat/galvanostat (VersaSTAT 3, Princeton Applied Research, Oak Ridge, TN, USA) with a standard three-electrode cell configuration. $\overline{\mathrm{MoS}_{2}} / \mathrm{ml}$-G films were used as the working electrode. $\mathrm{Ag} / \mathrm{AgCl} / \mathrm{KCl}(3 \mathrm{M})$ and $\mathrm{Pt}$ wire were used as the reference and counter electrode, respectively. An aqueous solution of $0.5 \mathrm{M}$ of $\mathrm{H}_{2} \mathrm{SO}_{4}$ was used as the electrolyte and was degassed using argon. Onset potentials were measured by extrapolating to zero current density the initial linear part of the V-I plot. The standard error of the measurement, based on three independent batches, was estimated to be $20 \%$.

\subsubsection{General procedure for the oxidative $\mathrm{C}-\mathrm{N}$ coupling}

Typically, 1.6 mol\% (total metal vs. substrate) of metal catalyst was added to a reaction mixture of benzimidazole $(0.1 \mathrm{mmol})$ in DMA $(2 \mathrm{~mL})$ inside a reinforced glass reactor. Then, the suspension was magnetically stirred, while purging with Ar and heated at $110{ }^{\circ} \mathrm{C}$ using a preheated silicone bath. Once the reaction temperature was reached, the system was closed and pressurized with $\operatorname{Ar}(2.5$ bar). Then, TBHP (5 M solution in decane) was introduced to the reaction mixture in two steps, $0.25 \mathrm{mmol}$ at the starting time, and $0.15 \mathrm{mmol}$ at $4 \mathrm{~h}$ reaction time. The temporal evolution of the reaction was followed by analyzing periodically known aliquots of the reaction mixture taken with a syringe provided with a filter $(0.2 \mu \mathrm{m})$ to remove the solid catalyst. The aliquots were immediately analyzed by GC. Yields were based on GC analysis of the reaction mixture containing weight amounts of indole as external 
standard. Experiments using $\mathrm{Fe}(\mathrm{CO})_{5}, \mathrm{FeCl}_{2}$ or $\mathrm{Fe}(\mathrm{OAc})_{3}$ as homogeneous catalysts were conducted in the same conditions as indicated above with 5.3, 3.4, $6.5 \mathrm{mg}$ for each of this catalyst, respectively, corresponding to $1.5 \mathrm{mg}$ of Fe. The amounts were taken by weighing the amounts in $\mathrm{mg}$ dissolving in $1 \mathrm{~mL}$ of DMA and taking $1 \mathrm{~mL}$ of the solution with a syringe. Formation of $\mathrm{C}-\mathrm{N}$ coupling product in yields below $5 \%$ was observed after $24 \mathrm{~h}$.

\subsubsection{Reuse experiment for the oxidative $\mathrm{C}-\mathrm{N}$ coupling}

Once the reaction performed as indicated in the point 8.2.4 was finished, the catalyst was recovered from the reaction mixture by filtration (Nylon filter, $0.2 \mu \mathrm{m}$ ), washed three times with DMA $(3 \times 10 \mathrm{~mL})$ and dried in an oven $\left(70^{\circ} \mathrm{C}\right)$ overnight. Then, the catalyst was used again in the next $\mathrm{C}-\mathrm{N}$ coupling reaction under the same conditions.

\subsubsection{Catalytic test procedure for the $\mathrm{CO}_{2}$ hydrogenation}

Experiments in the presence of $\mathrm{Fe}_{x} \mathrm{Co}_{y} @(N) G$ samples were carried out using a constant mass of $20 \mathrm{mg}$ of powdered catalyst in the absence of binder and a flow of 1 and $3 \mathrm{~mL} \mathrm{~min}-1$ for $\mathrm{CO}_{2}$ and $\mathrm{H}_{2}$ (pressure: 10 bar) respectively. For each sample, the catalytic tests were performed starting at $300{ }^{\circ} \mathrm{C}$ for $1 \mathrm{~h}$ and increasing the reactor temperature by $50{ }^{\circ} \mathrm{C}$ increments every $1 \mathrm{~h}$ up to $550{ }^{\circ} \mathrm{C}$ and, then, decreasing the temperature by $50{ }^{\circ} \mathrm{C}$ decrements every $1 \mathrm{~h}$ up to returning to $300{ }^{\circ} \mathrm{C}$. Thus, each experiment corresponds to 11 steps of $1 \mathrm{~h}$ of continuous reaction. The products during the reaction were analyzed by using GC. 


\subsection{Characterization techniques}

\subsubsection{Transmission electron microscopy (TEM)}

High resolution transmission electron microscopy (HR-TEM) is a technique that provides the image of a sample at the atomic scale (using a high-energy electron beam that interacts with the sample). Due to its resolution, it allows to study the characteristics of a material at a scale of a few nanometers or less. Currently the highest resolution is $0.8 \AA$, which has allowed the observation of individual atoms and structural defects. The theoretical principle under which an HR-TEM works is to measure the wave amplitude resulting from the interference between the incident electron and the surface of the sample. Transmission electron microscopy (TEM) images were taken here using a JEOL JEM-1010 microscope operating at $100 \mathrm{kV}$, while the HR-TEM was carried out with a JEOL JEM 2100F microscope operating with an acceleration voltage of $200 \mathrm{kV}$ coupled with an X-Max 80 energy dispersive X-ray detector (EDX) (Oxford instruments). The microscope is equipped with the STEM unit, the dark-field and high-angle field image detectors (HAADF) that facilitate the observation of phase contrast with different atomic number.

For the preparation of TEM samples of solids, a drop of a suspension of the material after sonicating the samples in an organic solvent (ethanol, methanol) for 1 $\mathrm{h}$ was added on a copper or nickel grid coated with a carbon film. For the preparation of TEM samples for films, small debris of the films were placed on the grid by scratching the films with a sharp knife and dipping the grid on the surface.

\subsubsection{Scanning electron microscopy (SEM)}

Scanning electron microscope (SEM) is an instrument that allows observation and surface characterization of inorganic and organic materials that are 
non-transparent to electrons. The technique gives morphological information about the particles of the analyzed material. SEM is used to acquire a series of images of the sample that are used to examine the morphology of microscopic areas, their homogeneity and to determine the particle size distribution, as well as a quantitative elemental analysis of the images obtained. The main characteristics of the SEM are its resolution ( $\sim 100 \AA)$, the depth of field that can give three-dimensional appearance to the images and the simple preparation of the samples. SEM images were taken with a JEOL JSM-5410 microscope, while the SEM images of emission of field (FESEM) were obtained with a Zeiss Ultra 55 instrument. The elemental analysis by energy dispersive X-ray spectroscopy (EDX) was performed with an Oxford instruments detector coupled to these microscopes. With fast ion bombardment (FIB) technique coupled to the FESEM, a sharp cut can be made in the film, allowing to obtain a cross-sectional image. The cut was done using focused beam of high energetic gallium ions operating at high currents.

The samples for SEM were prepared by adhering the specimens on a sample holder that is covered with a double-sided conductive tape. The non-conductive samples were previously metalized with a nanometric gold film by sputtering.

\subsubsection{Atomic force microscopy (AFM)}

AFM is a technique that allows to study physical characteristics of the surface of a sample or film and is especially useful for determining the thickness of thin films on atomically flat substrate, measuring the difference of height between the substrate and the surface of the film. The AFM measurements were performed with a Multimode Nanoscope $3 \mathrm{~A}$ instrument that has subnanometric vertical resolution and horizontal resolution of about $5 \mathrm{~nm}$. The samples were introduced into the equipment, scanning in "tapping" mode consisting in intermittent tip-sample contact. This procedure produces less alteration of the surface of the materials.

The graphene films deposited on quartz substrates containing or not 
nanoparticles are directly glued on a metal support of $1 \times 1 \mathrm{~cm}^{2}$ which is the suitable size for introducing the sample into the equipment. Images were obtained by scanning with the tip on the surface of samples. To prepare the samples for thickness measurement, graphene films were scratched mildly with a sharp knife without destroying the surface of substrates. The height information of the films can be obtained from the image focused on the scratch.

\subsubsection{Raman spectroscopy}

Raman spectroscopy is a very useful characterization technique for carbonaceous materials. Raman spectra can be recorded directly with the material to be analyzed either as powder or as film deposited on substrates, without the need for any sample pretreatment. Raman spectroscopy is based on the examination of scattered light from a material irradiated with a beam of monochromatic light. A small portion of the light is scattered inelastically, resulting in slight changes in the frequency. These changes are characteristic of the analyzed materials and independent of the frequency of the incident light. The Raman spectroscopy measurements were carried out using a Renishaw in Via Raman Microscope at room temperature using an argon ion laser of $514 \mathrm{~nm}$ as a source of excitation coupled to a microscope Leyca Optics that allows monitoring the sample exposed to the laser beam with a surface area resolution of $1 \times 1 \mu \mathrm{m}^{2}$. At each point of the sample, the Raman spectra were recorded in the region from 0 to $3500 \mathrm{~cm}^{-1}$, with a resolution $<4$ $\mathrm{cm}^{-1}$, performing 10 scans in a total accumulation time of $100 \mathrm{~s}$. The analysis requires the comparison and average of the spectra in several parts of the sample.

\subsubsection{X-ray diffraction (XRD)}

XRD is a characterization technique used to establish the crystallinity of the materials. It is a non-destructive technique so it allows the recovery of the sample. 
The XRD patterns in this Doctoral Thesis were obtained using a PANalytical Cubix-Pro diffractometer, which is equipped with a PANalytical X'Celerator detector. It employs a monochromatic X-ray radiation of $\mathrm{Cu} K \alpha\left(\Lambda_{1}=1.5406 \AA, \Lambda_{2}=1.5444 \AA, I_{2} / \mathrm{I}=0.5\right)$, a voltage of $45 \mathrm{kV}$ and a tube current of $40 \mathrm{~mA}$. The length of the goniometer arm is $200 \mathrm{~mm}$. The diffractograms are obtained at room temperature in the $2 \theta$ angle range between 2 and $90^{\circ}$, with a step increase of $0.02^{\circ}(2 \theta)$ in continuous mode. In addition, the width of the diffraction peaks is related to the distribution of the particle size. The calculation of average sizes of the nanoparticles by XRD patterns is based on the Scherrer equation:

$$
D=\frac{K \gamma}{B \cos \theta}
$$

where:

- $D$ is the calculated mean size of the ordered domains, which may be smaller or equal to the grain size;

- $\quad K$ is a dimensionless shape factor, with a typical value of about 0.9;

- $\quad Y$ is the $X$-ray wavelength;

- $B$ is the line broadening at half the maximum intensity (FWHM) (in radians);

- $\quad \vartheta$ is the Bragg angle (in degrees).

\subsubsection{X-ray photoelectron spectroscopy (XPS)}

XPS is a technique of characterization of surfaces that is commonly used to estimate the stoichiometry (with an error of approximately $10 \%$ ), chemical status and electronic structure of the elements that exist in a material. The XP spectra are obtained by irradiating with X-rays (usually from an anode of $\mathrm{Al}$ or $\mathrm{Mg}$ ), measuring at the same time the kinetic energy and the number of electrons that escape from the surface of the material. The XPS were taken in a SPECS spectrometer equipped with a Phoibos 150-9MCD detector using a non-monochromatic X-ray source of Al Ka $(1483.6 \mathrm{eV})$ operating at $50 \mathrm{~W}$. The samples were evacuated in a pre-chamber of the spectrometer at $10^{-9}$ mbar. Quantification and treatment of the spectra were carried 
out with the software CASA, and the correction is made based on the carbon signal C1s, whose bonding energy corresponds to the value of $284.5 \mathrm{eV}$.

\subsubsection{Combustion elemental analysis}

Combustioin elemental analysis is a technique that allows determining the percentages of $\mathrm{C}, \mathrm{H}, \mathrm{N}$ and $\mathrm{S}$ present in the sample. It is a destructive micro-combustion technique. The sample with a weight of $2-4 \mathrm{mg}$ is subjected to thermal oxidation at temperatures between $166-1800{ }^{\circ} \mathrm{C}$ in an $\mathrm{O}_{2}$ environment, resulting in the total conversion of the components to $\mathrm{CO}_{2}$ (carbon), $\mathrm{H}_{2} \mathrm{O}$ (hydrogen) $\mathrm{NO}$ or $\mathrm{NO}_{2}$ (nitrogen), and $\mathrm{SO}_{2}$ (sulfur). The contents of these elements can be determined by quantitative analysis of the resulting combustion products. The analyzer that has been used to carry out the analysis in the present Doctoral Thesis is Euro EA3000 Elemental Analyzer (EuroVector), using sulfanilamide as a reference to check the accuracy of measurements. The accuracy of the instrument is on the order of $0.01 \%$.

\subsubsection{ICP-OES plasma spectroscopy}

The inductively coupled plasma optical emission spectrometry (ICP-OES) is used to determine the composition and content of metals and some other elements in samples. The technique is based on the excitation of atoms in solution that is nebulized in high temperature plasma. When the excited atoms fall to the ground state, a photon of characteristic energy for each atom is emitted. The concentration of the element in the sample is determined from the intensity of this emission, comparing the intensity with the values from the calibration curve for each element. The samples have been analyzed on a Varian 715-ES ICP-Optical Emission Spectrophotometer.

The general procedure used to analyze the samples is as follows: $20 \mathrm{mg}$ of a 
sample was dissolved by treatment of the sample with $10 \mathrm{~mL}$ of aqua regia solution $\left(\mathrm{HCl} / \mathrm{HNO}_{3} 3: 1\right)$ for $3 \mathrm{~h}$ at room temperature. After filtering the resulting suspension to separate any possible remaining solid waste, the solution was diluted to $50 \mathrm{~mL}$ with milli-Q water. Then the solution was injected into the spectrophotometer.

\subsubsection{Thermogravimetric analysis (TGA)}

This type of analysis aims to study the processes of decomposition of organic matter and its stability depending on the temperature and atmosphere to which it is exposed. TGA were performed with a Mettler Toledo TGA/SDTA 851e device in the temperature range from 20 to $900{ }^{\circ} \mathrm{C}$ at a speed of $10{ }^{\circ} \mathrm{C} \mathrm{min}-1$ and an air flow of 20 $\mathrm{mL} \mathrm{min}^{-1}$. The amount of sample required for an analysis is approximately $1 \mathrm{mg}$.

\subsubsection{Gas Chromatography (GC)}

$\mathrm{GC}$ is one of the most used techniques for analysis of the composition of volatile compounds in a sample. Common with all the chromatographic techniques, a gas chromatograph contains a stationary phase consisting frequently of a polysiloxane that coats as a thin micrometric layer a capillary column and a mobile phase responsible for dragging the analyte or compound to be analyzed from the injector to the detector through the stationary phase. Analyzes by GC in this project, were carried out on a Thermo Trace GC Ultra instrument equipped with a TraceGOLD TG-5SilMS column (30 $\mathrm{m} \times 0.25 \mathrm{~mm} \times 0.25 \mu \mathrm{m})$ and with a flame ionization detector (FID). Taking into account that the ionization detectors give a signal proportional to the mass of the product analyzed by determining the chromatographic peak area and calibrating the response of each analyte with respect to a standard, it is possible to calculate the concentration of each component in the reaction mixture. 


\subsubsection{Gas Chromatography coupled to mass spectrometry (GC-MS)}

The gas chromatography technique coupled to mass spectrometry (GC-MS) consists of the combination of a gas chromatograph and a mass spectrometer that acts as detector. The mass spectrometry (MS) is a widely used microanalytical technique for identification of compounds and to elucidate their possible structures. Gas chromatography-mass spectrometry analysis in the project were carried out in a Thermo Trace GC Ultra instrument equipped with a column TraceGOLD TG-5SilMS (30 $\mathrm{m} \times 0.25 \mathrm{~mm} \times 0.25 \mu \mathrm{m})$ and coupled to a mass spectrometer Thermo DSQ quadrupole.

\subsubsection{Optical microscopy}

This technique is used primarily for the observation of objects in the scale of micrometers. Pictures in the dark field can be taken after exciting the sample with three different wavelengths $(400,450$ and $500 \mathrm{~nm})$, allowing to observe the fluorescence emission of the object. The apparatus used was a Leica DM 4000B optical microscope connected to an ebq 100 ISOLATED power module, whose resolution is around a micron.

\subsubsection{Brunauer-Emmett-Teller (BET) surface area analysis}

The BET surface area analysis is based on the physical adsorption of gas molecules on the surface of the solids and is an important technique to determine the special surface areas of materials. The gases that do not have the chemical reaction with the surface of materials are used as adsorbates to quantify specific surface area. The most commomly used gas for the measurement is $N_{2}$. In the present work, the surface areas of the solids were determined by the Brunauer-Emmet-Teller method (BET) analyzing the nitrogen adsorption isotherms 
measured at $77 \mathrm{~K}$ of the previously degassed samples in a Micromeritics ASAP-2420 equipment. 
Chapter 9

Conclusions 

Overall the results achieved in the present Doctoral Thesis represent a notable jump in the development of graphene-based heterojunctions by applying natural polysaccharides as graphene precursors. Thus, it has been shown that the process can serve to prepare conductive films as well as a large variety of heterojunctions of these defective graphenes with other different 2D materials. It has also been shown that there is a range of experimental conditions in which the junctions of defective graphenes and metal nanoparticles can be formed, even for these non-noble metals that could form carbides. More specifically the conclusions of each of the Chapters of the Thesis where original research has been carried out are the following:

1. It has been shown that the presence of hydrogen in proportions about 5 or $10 \%$ increases the quality of the resulting defective graphene according to Raman spectroscopy and there is a decrease in the proportion of the residual oxygen of the material. This quality improvement is reflected in some increase in the electrical conductivity and the photoelectric activity of the films, although there is still room for improvement.

2. It has been shown that it is possible to form films of heterojunction of boron nitride and defective graphene in one-step pyrolysis of ammonium borate adsorbed on chitosan. The resulting defective graphene-boron nitride heterojunction prepared in this way does not exhibit, however, higher electrical conductivity than the values measured for the corresponding defective graphene on quartz. It has been observed, however, that the defective graphene-boron nitride heterojunction works as microcapacitor upon charge and discharge cycle.

3. It has been shown that the one-step pyrolysis of chitosan containing diammonium molybdotetrasulfide can be used to form films of defective graphene/ oriented $\mathrm{MoS}_{2}$ heterojunction. The resulting film exhibits electrocatalytic activity for hydrogen generation, although it was observed that there is a dispersion on the performance for batches prepared following the same procedure.

4. Pyrolysis of chitosan powders containing iron or cobalt acetate or mixture of 
both results in iron or cobalt or the corresponding alloys as nanoparticles embedded within a graphitic carbon residue. These carbons embedded metal nanoparticles exhibit activity as heterogeneous catalysts for the oxidative coupling of $\mathrm{N}$-methyl amides and $\mathrm{N}-\mathrm{H}$ aromatic heterocycles. The reaction requires an excess of tert-butylhydroperoxide, reaching higher yields upon addition of the reagent in two steps.

5. It has been shown that Fe-Co alloy nanoparticles embedded within a graphitic carbon matrix prepared by pyrolysis of chitosan beads with adsorbed iron and cobalt acetates catalyse hydrogenation of $\mathrm{CO}_{2}$ to form isobutane, reaching a high selectivity to methane (above 92\%) at a high $\mathrm{CO}_{2}$ conversion (about 87\%). Theoretical calculations show that the possible reason for the remarkable activity is the strong interaction between the metals and defective graphene, resulting in a charge transfer and differentiation among the charge density of the various atoms in the cluster.

The candidate's contributions to Chapters 3-7:

Chapter 3: the main works are from the candidate.

Chapter 4: the candidate took part in the synthesis of the materials and performed some of the sample characterizations.

Chapter 5: the main works are from the candidate, except the electrochemical tests.

Chapter 6: the main works are from the candidate, except the analysis of reaction mechanism.

Chapter 7: the candidate synthesized the samples and performed some of the sample characterizations. 
Abstracts 



\section{Abstract}

In this Doctoral Thesis, the heterojunctions of graphenes with other 2D materials and metal nanoparticles, including (N)graphene/h-BN, graphene/ $\mathrm{MoS}_{2}$ and $\mathrm{Fe} / \mathrm{Co}$ deposited graphene, were synthesized based on using natural polysaccharides as graphene precursors. These materials were characterized using various analytical methods and were tested for oxidative $\mathrm{C}-\mathrm{N}$ coupling of amides, $\mathrm{CO}_{2}$ hydrogenation or physical and photoelectric catalytic application.

In the first stage of the thesis, the influence of temperature and the presence of $\mathrm{H}_{2}$ during pyrolysis on the quality of graphene was studied. It was observed that a significant decrease in the density of defects related to the presence of residual oxygen can be achieved when the produce was performed at the optimal temperature $\left(1100{ }^{\circ} \mathrm{C}\right)$ under a low percentage of $\mathrm{H}_{2}(5 \%)$. This improvement in the quality of the resulting defective graphene was reflected in a decrease in the electrical resistance and increased photoelectric activity.

In the case of $\mathrm{N}$-doped graphene/h-BN heterostructures, it has been revealed that a spontaneous segregation $(\mathrm{N})$ graphene and boron nitride layers took place during the pyrolysis. Although the resulting heterostructures did not show an improvement in the conductivity, the material could behavior as capacitor storing charge in the range of positive voltages.

Graphene/MoS 2 was prepared by pyrolysis of alginic acid containing adsorbed $\left(\mathrm{NH}_{4}\right)_{2} \mathrm{MoS}_{4}$. The $\mathrm{MoS}_{2}$ nanoparticles exhibited a preferential 002 facet orientation, as a result of the template effect of graphene layers. This material exhibited activity for $\mathrm{H}_{2}$ evolution reaction, although some variation of the electrocatalytic activity has been observed from batch to batch.

$\mathrm{Fe}$, Co NPs or Fe-Co alloys embedded in carbonaceous matrix were also prepared by pyrolysis of chitosan powders containing $\mathrm{Fe}^{2+}$ and $\mathrm{Co}^{2+}$ ions at $900{ }^{\circ} \mathrm{C}$ under Ar atmosphere and used for the oxidative C-N coupling of amides and 
aromatic N-H compounds. It was observed that sequential addition of two aliquots of tert-butyl hydroperoxide (TBHP) in an excess of $\mathrm{N}, \mathrm{N}$-dimethylacetamide (DMA) as solvent afforded the corresponding coupling product in high yields, and the most efficient catalyst was FeNP@C with high reusability and a wide scope.

Finally, beads of graphitic carbon matrix containing Fe, Co NPs or Fe-Co alloys were sequentially synthesized by one-step pyrolysis at $900{ }^{\circ} \mathrm{C}$ of chitosan beads having adsorbed iron and cobalt acetates. The best sample, Fe-Co alloy/G (Fe/Co about 0.4 ), showed high activity for the hydrogenation of $\mathrm{CO}_{2}$ to isobutane with a selectivity higher than $92 \%$ and a $\mathrm{CO}_{2}$ conversion about $87 \%$. 
Resumen

En esta Tesis Doctoral, las heterouniones de grafeno con otros materiales 2D y nanopartículas metálicas, incluyendo (N)grafeno/h-BN, grafeno/ $\mathrm{MoS}_{2}$ y grafeno depositado $\mathrm{Fe} / \mathrm{Co}$, se sintetizaron en base al uso de polisacáridos naturales como precursors de grafeno. Estos materials se caracterizaron usando diversos métodos analíticos y se ensayaron para determinar el acoplamiento $\mathrm{C}-\mathrm{N}$ oxidativo de las amidas, la hidrogenación de $\mathrm{CO}_{2}$ o la aplicación catalítica fotoeléctrica y física.

En la primera etapa de la tesis, se estudió la influencia de la temperatura y la presencia de $\mathrm{H}_{2}$ durante la pirólisis en la calidad del grafeno. Se observó que una disminución significativa en la densidad de defectos relacionados con la presencia de oxígeno residual se puede lograr cuando el producto se preparó a la temperatura óptima $\left(1100{ }^{\circ} \mathrm{C}\right)$ bajo un bajo porcentaje de $\mathrm{H}_{2}(5 \%)$. Esta mejora en la calidad del grafeno defectuoso resultante se reflejó en una disminución de la resistencia eléctrica y una mayor actividad fotoeléctrica.

En el caso de las heteroestructuras de grafeno dopadas con $\mathrm{N} / \mathrm{h}-\mathrm{BN}$, se ha revelado que se produjeron capas de segregación espontánea $(\mathrm{N})$ grafeno y nitruro de boro durante la pirólisis. Aunque las heteroestructuras resultantes no mostraron una mejora en la conductividad, el material podría comportarse como un condensador que almacena carga en el rango de voltajes positivos.

El grafeno/ $\mathrm{MoS}_{2}$ se preparó por pirólisis de ácido algínico que contenía $\left(\mathrm{NH}_{4}\right)_{2} \mathrm{MoS}_{4}$ adsorbido. Las nanopartículas de $\mathrm{MoS}_{2}$ exhibieron una orientación preferencial en la cara 002, como resultado del efecto de plantilla de las capas de grafeno. Este material exhibió actividad para la reacción de evolución $\mathrm{H}_{2}$, aunque se ha observado alguna variación de la actividad electrocatalítica de un lote a otro.

También se prepararon $\mathrm{Fe}$, Co NP o aleaciones Fe-Co incrustadas en matriz carbonosa por pirólisis de polvos de quitosano que contenían iones $\mathrm{Fe}^{2+}$ y $\mathrm{Co}^{2+}$ a 900 ${ }^{\circ} \mathrm{C}$ en atmósfera de Ar y se usaron para el acoplamiento oxidativo de $\mathrm{C}-\mathrm{N}$ de amidas y compuestos aromáticos de N-H. Se observó que la adición secuencial de dos alícuotas 
de hidroperóxido de terc-butilo (TBHP) en un exceso de $\mathrm{N}, \mathrm{N}$-dimetilacetamida (DMA) como disolvente proporcionaba el correspondiente producto de acoplamiento en altos rendimientos, y el catalizador más eficiente era FeNP@C con alta reutilización y un amplio alcance.

Finalmente, las perlas de matriz de carbono grafítico que contienen Fe, Co NPs o aleaciones de Fe-Co se sintetizaron secuencialmente mediante pirólisis en una etapa a $900{ }^{\circ} \mathrm{C}$ de perlas de quitosano que tenían acetatos de hierro y cobalto adsorbidos. La mejor muestra, Fe-Co aleación/G (Fe/Co alrededor de 0.4), mostró alta actividad para la hidrogenación de $\mathrm{CO}_{2}$ a isobutano con una selectividad superior al $92 \%$ y una conversión de $\mathrm{CO}_{2}$ de aproximadamente el $87 \%$. 
Resum

En esta Tesi Doctoral, les heterounions de grafeno amb altres materials 2D i nanopartícules metàl-liques, incloent $(\mathrm{N})$ grafé/h-BN, grafé/MoS $\mathrm{S}_{2}$ i grafé depositat $\mathrm{Fe} / \mathrm{Co}$, es van sintetitzar basant-se en l'ús de polisacàrids naturals com precursors de grafé. Estos materials es van caracteritzar usant diversos mètodes analítics i es van assajar per a determinar l'adaptament $\mathrm{C}-\mathrm{N}$ oxidatiu de les amides, la hidrogenació de $\mathrm{CO}_{2}$ o l'aplicació catalítica fotoelèctrica i física.

En la primera etapa de la tesi, es va estudiar la influència de la temperatura i la presència de $\mathrm{H}_{2}$ durant la piròlisi en la qualitat del grafé. Es va observar que una disminució significativa en la densitat de defectes relacionats amb la presència d'oxigen residual es pot aconseguir quan el producte es va preparar a la temperatura òptima $\left(1100{ }^{\circ} \mathrm{C}\right)$ davall un baix percentatge de $\mathrm{H}_{2}(5 \%)$. Esta millora en la qualitat del grafé defectuós resultant es va reflectir en una disminució de la resistència elèctrica i una major activitat fotoelèctrica.

En el cas de les heteroestructures de grafé dopades amb N/h-BN, s'ha revelat que es van produir capes de segregació espontània $(\mathrm{N})$ grafé i nitrur de bor durant la piròlisi. Encara que les heteroestructures resultants no van mostrar una millora en la conductivitat, el material podria comportar-se com un condensador que emmagatzema càrrega en el rang de voltatges positius.

El grafé/MoS $\mathrm{Mos}_{2}$ va preparar per piròlisi d'àcid algínic que contenia $\left(\mathrm{NH}_{4}\right)_{2} \mathrm{MoS}_{4}$ adsorbit. Les nanopartícules de $\mathrm{MoS}_{2}$ van exhibir una orientació preferencial en la cara 002, com resultat de l'efecte de plantilla de les capes de grafé. Este material va exhibir activitat per a la reacció d'evolució $\mathrm{H}_{2}$, encara que s'ha observat alguna variació de l'activitat electrocatalítica d'un lot a un altre.

També es van preparar $\mathrm{Fe}$, Co NP o aliatges $\mathrm{Fe}-\mathrm{Co}$ incrustades en matriu carbonosa per piròlisi de pols de quitosano que contenien ions $\mathrm{Fe}^{2+}$ i $\mathrm{Co}^{2+}$ a $900{ }^{\circ} \mathrm{C}$ en atmosfera d'Ar i es van usar per a l'acoblament oxidatiu de C-N d'amides i compostos aromàtics de NH. Es va observar que l'addició seqüencial de dos alíquotes de 
hidroperóxid de terc-butil (TBHP) en un excés de N,N-dimetilacetamida (DMA) com a dissolvent proporcionava el corresponent producte d'acoblament en alts rendiments, i el catalitzador més eficient era FeNP@C amb alta reutilització i un ampli abast.

Finalment, les perles de matriu de carboni grafític que contenen Fe, Co NPs o aliatges de $\mathrm{Fe}-\mathrm{Co}$ es van sintetitzar seqüencialment per mitjà de piròlisi en una etapa a $900{ }^{\circ} \mathrm{C}$ de perles de quitosano que tenien acetats de ferro i cobalt adsorbits. La millor mostra, Fe-Co aliatge/G (Fe/Co al voltant de 0.4), va mostrar alta activitat per a la hidrogenació de $\mathrm{CO}_{2}$ a isobutà amb una selectivitat superior al 92\% i una conversió de $\mathrm{CO}_{2}$ d'aproximadament el $87 \%$. 


\section{List of publications}

The following publications form part of the present Doctoral Thesis:

\section{Chapter 3}

Quality Improvement of Defective Graphene From Biomass.

He, Jinbao; Primo, Ana; Garcia, Hermenegildo.

Under revision

\section{Chapter 4}

Catalyst-free One Step Synthesis of Large Area Vertically Stacked N-doped Graphene-Boron Nitride Heterostructures from Biomass Source.

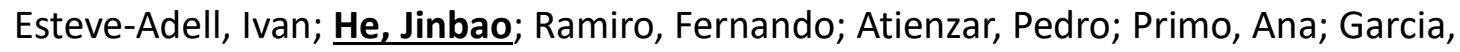
Hermenegildo.

Published in: Nanoscale 2018, 10(9), 4391-4397.

\section{Chapter 5}

One-Step Preparation of Large Area Films of Oriented $\mathrm{MoS}_{2}$ Nanoparticles on Multilayer Graphene and its Electrocatalytic Activity for Hydrogen Evolution.

He, Jinbao; Fernandez, Cristina; Primo, Ana; Garcia, Hermenegildo.

Published in: Materials 2018, 11(1), 168.

\section{Chapter 6}

Iron Nanoparticles Embedded in Graphitic Carbon Matrix as Heterogeneous Catalysts for the Oxidative $\mathrm{C}-\mathrm{N}$ Coupling of Aromatic N-H Compounds and Amides. He, Jinbao; Dhakshinamoorthy, Amarajothi; Primo, Ana; Garcia, Hermenegildo.

Published in: ChemCatChem 2017, 9(15), 3003-3012.

\section{Chapter 7}


Extremely High Selectivity Towards Isobutane in $\mathrm{CO}_{2}$ Hydrogenation Catalyzed by Fe-Co Alloy Nanoparticles Embedded in Graphitic Carbon Matrix.

Primo, Ana; He, Jinbao; Franconetti, Antonio; Jurca, Bogdan; Cojocaru, Bogdan; Apostol, Nicoleta G.; Ruxandra, Costescu M.; Cristian, Teodorescu M.; Parvulescu, Vasile I; Garcia, Hermenegildo.

Under revision

Other publications:

-Defective Graphene as Metal Free Catalyst for Chemoselective Olefin Hydrogenation by Hydrazine.

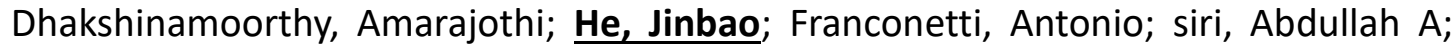
Primo, Ana; Garcia, Hermenegildo.

Published in: Catalysis Science \& Technology 2018, 8(6), 1589-1598.

$-\mathrm{CO}_{2}$ Methanation Catalyzed by Oriented $\mathrm{MoS}_{2}$ Nanoplatelets Supported on Few Layers Graphene.

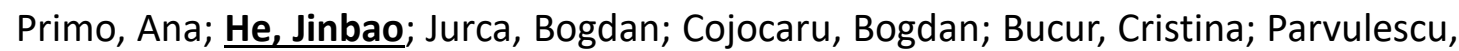
Vasile I; Garcia, Hermenegildo.

Under revision

$-\mathrm{CO}_{2}$ Methanation Catalyzed by Nickel and Copper Nanoparticles Supported on Defective Graphene

He, Jinbao; Primo, Ana; Garcia, Hermenegildo; Parvulescu, Vasile I.

Under revision

-Highly Selective Photocatalytic Benzene Hydroxylation to Phenol Using Surface-Modified $\mathrm{Cu}_{2} \mathrm{O}$ Supported on Graphene

He, Jinbao; Zhang, Ming; Primo, Ana; Garcia, Hermenegildo; Li, Zhaohui.

Published in: Journal of Materials Chemistry A, 2018. 